Linköping studies in science and technology. Dissertations.

No. 1617

\title{
Modeling and Diagnosis of Friction and Wear in Industrial Robots
}

\section{André Carvalho Bittencourt}

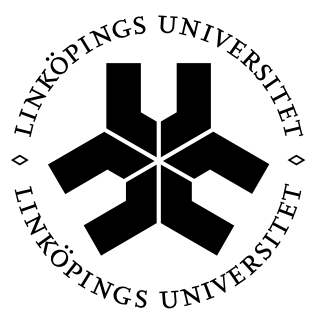

Department of Electrical Engineering

Linköping University, SE-581 83 Linköping, Sweden

Linköping 2014 
Cover illustration: Friction curves for different values of temperature, load and wear. The RGB color used in each curve corresponds to the value of temperature (red, $[30-80]^{\circ} \mathrm{C}$ ), load (green, $[0-100] \%$ ) and wear (blue, $[0-50] \%$ ).

Linköping studies in science and technology. Dissertations.

No. 1617

\title{
Modeling and Diagnosis of Friction and Wear in Industrial Robots
}

André Carvalho Bittencourt

\author{
andrecbeisy.liu.se \\ www. control.isy.liu.se \\ Division of Automatic Control \\ Department of Electrical Engineering \\ Linköping University \\ SE-581 83 Linköping \\ Sweden
}

ISBN 978-91-7519-251-2

ISSN 0345-7524

Copyright @ 2014 André Carvalho Bittencourt

Printed by LiU-Tryck, Linköping, Sweden 2014 
To the memory of my brother. 



\section{Abstract}

High availability and low operational costs are critical for industrial systems. While industrial equipments are designed to endure several years of uninterrupted operation, their behavior and performance will eventually deteriorate over time. To support service and operation decisions, it is important to devise methods to infer the condition of equipments from available data.

The monitoring of industrial robots is an important problem considered in this thesis. The main focus is on the design of methods for the detection of excessive degradations due to wear in a robot joint. Since wear is related to friction, an important idea for the proposed solutions is to analyze the behavior of friction in the joint to infer about wear. Based on a proposed friction model and friction data collected from dedicated experiments, a method is suggested to estimate wear-related effects to friction. As it is shown, the achieved estimates allow for a clear distinction of the wear effects even in the presence of large variations to friction associated to other variables, such as temperature and load.

In automated manufacturing, a continuous and repeatable operation of equipments is important to achieve production requirements. Such repetitive behavior of equipments is explored to define a data-driven approach to diagnosis. Considering data collected from a repetitive operation, an abnormality is inferred by comparing nominal against monitored data in the distribution domain. The approach is demonstrated with successful applications for the diagnosis of wear in industrial robots and gear faults in a rotating machine.

Because only limited knowledge can be embedded in a fault detection method, it is important to evaluate solutions in scenarios of practical relevance. A simulation based framework is proposed that allows for determination of which variables affect a fault detection method the most and how these variables delimit the effectiveness of the solution. Based on an average performance criterion, an approach is also suggested for a direct comparison of different methods. The ideas are illustrated for the robotics application, revealing properties of the problem and of different fault detection solutions.

An important task in fault diagnosis is a correct determination of presence of a condition change. An early and reliable detection of an abnormality is important to support service, giving enough time to perform maintenance and avoid downtime. Data-driven methods are proposed for anomaly detection that only require availability of nominal data and minimal/meaningful specification parameters from the user. Estimates of the detection uncertainties are also possible, supporting higher level service decisions. The approach is illustrated with simulations and real data examples including the robotics application. 



\section{Populärvetenskaplig sammanfattning}

För industriella system är både hög tillgänglighet och låga driftskostnader avgörande. Industriella system är oftast utformad för att klara flera års oavbruten drift, men över tid kommer beteendet och prestandan så småningom att förändras. Det är därför viktigt att ta fram metoder som kan extrahera information från tillgänglig data och dra slutsatser om systemets beteende, som i sin tur används som stöd för beslut angående systemets fortsatta drift.

Denna avhandling handlar om utformning och utvärdering av diagnostiska metoder för att stödja tids- och kostnadseffektiva beslut angående den fortsatta driften för systemet. I synnerhet studeras problemet med att upptäcka för höga nivåer av slitage i respektive led för en industrirobot. Eftersom slitage påverkar friktionen kan det vara en bra idé att analysera friktionen för att uppskatta hur stort slitage som har uppkommit. Baserat på en föreslagen friktionsmodell och friktionsdata från specialanpassade experiment föreslås en metod för att uppskatta slitagets omfattning. Metoden försöker anpassa modellen så att sannolikheten att mätningarna kommer från den föreslagna modellen maximeras. Det visar sig att tillförlitliga beräkningar av slitaget kan uppnås även vid stora variationer $\mathrm{i}$ belastningen på roboten samt temperaturen i robotens leder, vilket gör det möjligt att planera underhåll för roboten innan den går sönder.

Vidare undersöks hur ett systems repetitiva beteende, som är vanligt inom automatiserad tillverkning, kan utnyttjas för att skapa en metod för diagnos som endast använder befintlig data utan hjälp av någon modell. Med hjälp av data som har samlats in från en repetitiv process kan en förändring av processen upptäckas genom att jämföra data från systemet i felfri drift och befintlig drift. Metoden som föreslås utnyttjar den empiriska sannolikhetsfördelningen för systemet i felfri respektive befintlig drift. Det visar sig att metoden med framgång kan detektera slitage i lederna för en industrirobot samt växelfel i en roterande mekanism.

I avhandlingen föreslås också metoder för feldetektering. Testet går ut på att man jämför två hypoteser mot varandra genom ett statistiskt ramverk. För att upptäcka en förändring av ett system är det naturligt att de två hypoteserna motsvarar ett system utan fel respektive ett system med fel. Det enda som förutsätts är att data från systemet utan fel är tillgängligt. En annan viktig del är att kunna jämföra olika diagnosmetoder för att se vilken som passar bäst till det aktuella problemet. Ett ramverk baserat på simuleringar har därför föreslagits för utvärdering av diagnosmetoder. Ramverket kan användas för att avgöra vilka variabler som påverkar metoden mest, hur man jämför olika metoder samt hur man bestämmer det effektiva användningsområdet för respektive metod. De föreslagna diagnosmetoderna och ramverket för utvärdering av diagnosmetoderna är generella men illustreras i avhandlingen på tillämpningar för industrirobotar. 



\section{Acknowledgments}

I would like to thank my supervisor Svante Gunnarsson for the guidance through these years, always gentle and prompt in my inquiries. Special thanks also to my co-supervisors Mikael Norrlöf and Erik Wernholt for the invaluable input. Thank you Lennart Ljung and Svante Gunnarsson for accepting me in the group and Shiva Sander Tavallaey for inviting me to graduate education. Being a graduate student at the ISY/RT group has been a remarkable experience and I would like to express my gratitude to everyone behind our organizational structure. To mention some, thank you Lennart Ljung and Svante Gunnarsson for your leadership; thank you Torkel Glad and Johan Löfberg for your roles in our educational programs; thank you Martin Enqvist for your availability and kindness; thank you Ulla Salaneck and Ninna Stensgård for the administrative support; thanks to all of our gurus, specially the ones behind our LTEX thesis template, Gustav Hendeby and Henrik Tidefelt. Special thanks for the people that helped me reviewing this thesis, Svante Gunnarsson, Mikael Norrlöf, Patrik Axelsson, Daniel Eriksson and Emre Özkan.

The close collaboration with ABB was very important for the achievements in this thesis. ABB not only supported me financially, via VINNOVA's Industry Excellence Center LINK-SIC, but also with expertise, guidance and friendship. Shiva Sander Tavallaey played a central role in all stages of the work, before, during and after; your dear guidance and kindness have been highly esteemed. Special thanks to Mikael Norrlöf, Kari Saarinen, Hans Andersson, Torgny Brogård and Shiva for all the fruitful discussions and the invaluable input. Thank you Niclas Sjöstrand for inviting me to ABB in 2007, event which is likely to have sparked much of this, and for inviting me again in 2012. Thank you Alf Isaksson and Krister Forsman from Perstorp for our collaborations outside the robotics landscape. Thank you all for helping me feel home at ABB.

The arduous and long journey towards a PhD was eased by the presence of good friends in my live. I was lucky enough to start together with RT's indisputable host, Sina Khoshfetrat Pakazad; your friendship has been an invaluable gift during this period, thanks for everything! Thank you Daniel Ankelhed, Jonas Linder and Patrik Axelsson for your patience and company as my office mates. Thank you Tohid Ardeshiri for always keeping an eye on me, but also for your generosity and unlimited excitement for any blow of wind. Speaking of bananas, thanks Karl Granström for showing me how to ride a mini motorcycle and for valuing my word more than I do sometimes, I will always regard you highly. Speaking of motorcycles, thank you Johan Löfberg, a.k.a. JLö, for the chance to enjoy riding again on your spare KTM, hopefully I will be riding my own soon. Probably sooner than the time it will take me to forget some of the memories from our planning meetings; thanks for that Fredrik Lindsten and for being a great partner in the misbehaving during the after hours. Unlike Emre Özkan, who spots bad ideas and moments right away with his telepathic skills; thank you for your brotherly friendship and for saving my life in Sheffield. Had you not been there at the right moment, I would not have had the chance to run long distances with 
Martin Skoglund, or to ski, hike, climb and drive $1500 \mathrm{~km}$ across Scandinavia with Hanna Nyqvist and Per, who are great people but have a strange taste for food. Unlike Marek Syldatk, who seems to have a taste for everything and has never tasted something that was not delicious; thanks for your loyal friendship and for all the beers we shared as flatmates. Speaking of beers, thanks Jonas Linder for the clockwork timing for Fredagspuben, after skis and after works. The after parties are naturally acknowledged to Clas Veibäck and Isak Nielsen, just as it should be acknowledged that Niklas Wahlström is the king's clarinet but holds the crown at the dance-floor. Thank you George Mathai for all the philosophical ventures, Michael Roth for keeping it simple and Patrik Axelsson for keeping it dependable; thanks for all the help with the PhD checklist PAx. Thank you Peter Rosander, Saikat Saha, Tianshi Chen, Henrik Ohlsson, Umut Orguner, Manon Kok, Carsten Fritsche, Daniel Petersson, Ylva Jung, Lubos Váci, Zoran Sjanic, Gustav Hendeby, Johan Dahlin and everyone else for all the moments we shared.

Obrigado pai, mãe e irmão pelo suporte e amor incondicionais. Admiro e amo cada de um vocês e espero poder estar mais presente daqui pra frente. Essa conquista é um simples reflexo da presença de vocês em minha vida. Um grande abraço e beijo.

Thank you Alicia for all the hugs in the morning, without warning. As you turn these pages, a new chapter stages. It is you and me again, no reason to abstain. Yours, 


\section{Contents}

$\begin{array}{lll}\text { Notation } & \text { XV }\end{array}$

1 Introduction 1

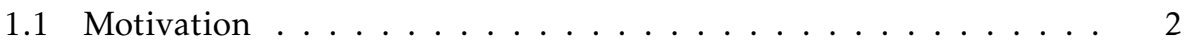

1.2 Research Goals and Approach . . . . . . . . . . . . . . 3

1.3 Thesis Outline . . . . . . . . . . . . . . . . 4

1.3.1 Background on the publications ........... 5

1.3.2 Relevant and additional work ........... 8

\section{Background}

2 Basics of Industrial Robotics 11

2.1 Actuators and Sensors . . . . . . . . . . . . . . . . . 13

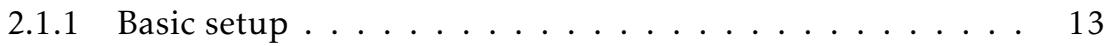

2.1.2 Application dependent sensors . . . . . . . . . . . 14

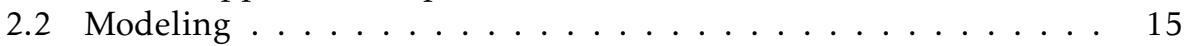

2.2 .1 Kinematics . . . . . . . . . . . . . . 15

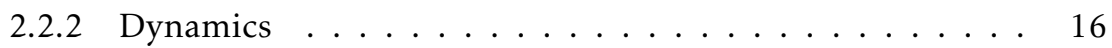

2.3 Identification . . . . . . . . . . . . . . . . . . 18

2.4 Reference Generation and Control . . . . . . . . . . . . . . . . 19

2.5 Summary and Connections . . . . . . . . . . . . . 22

3 Joint Friction and Wear 23

3.1 Basics of Tribology . . . . . . . . . . . . . . . . . . 24

3.2 Friction Dependencies in Robot joints . . . . . . . . . . . 25

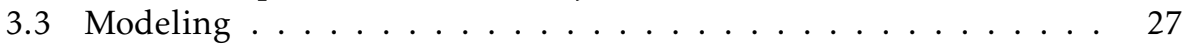

3.4 Summary and Connections . . . . . . . . . . . . . 29

4 Basics of Fault Diagnosis 31

4.1 Overview of Fault Diagnosis . . . . . . . . . . . . . . . 31

4.1.1 Fault detection . . . . . . . . . . . . . . 32

4.1.2 Models of systems and faults ........... 34 
4.2 Fault Detection Algorithms . . . . . . . . . . . . . . 36

4.2.1 Parameter estimation . . . . . . . . . . . . . 36

4.2.2 Signal-driven methods . . . . . . . . . . . . . 40

4.2.3 Data-driven methods ............... . . . . 41

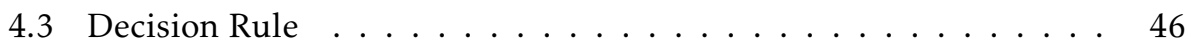

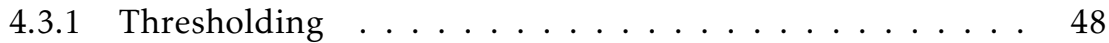

4.3.2 Likelihood ratio tests . . . . . . . . . . . . . 44 49

4.3.3 Statistical significance tests ............ 50

4.3.4 Compromises between errors and time of detection . . . . 51

4.4 Summary and Connections . . . . . . . . . . . . 52

5 Conclusions and Discussion $\mathbf{5 5}$

5.1 Conclusions of Part I . . . . . . . . . . . . . 55

5.2 Summary and Discussion for Part II . . . . . . . . . . . . 56

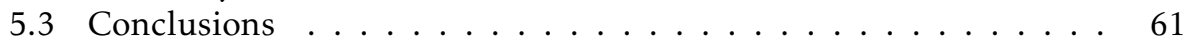

5.4 Recommendations for Future Research . . . . . . . . . . . . . 62

$\begin{array}{ll}\text { Bibliography } & 65\end{array}$

\section{Publications}

A Friction in a Robot Joint $\quad 75$

1 Introduction ............................ 77

2 Identification of Friction Models . . . . . . . . . . . . . . . . 82

$2.1 \quad$ Covariance estimate . . . . . . . . . . . . 83

3 Basics of Friction Phenomena in a Robot Joint . . . . . . . . . . . . 84

3.1 A procedure to estimate friction at a fixed speed level . . . 84

3.2 Modeling of velocity dependencies . . . . . . . . . . 86

4 Empirically Motivated Modeling ............... 88

4.1 Guidelines for the experiments . . . . . . . . . . . 88

4.2 Effects of joint angles ................. 89

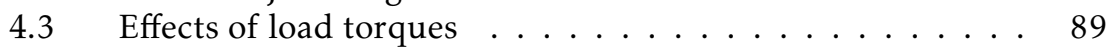

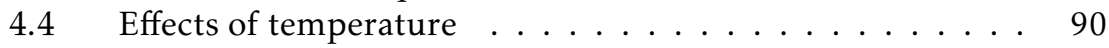

4.5 A complete model . . . . . . . . . . . . . 95

4.6 Validation ................... 95

5 Conclusions and Further Research . . . . . . . . . . . . . . . 97

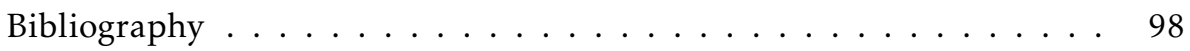

B Modeling and Identification of Wear in a Robot Joint 101

1 Introduction . . . . . . . . . . . . . . . 103

2 Steady-State Friction in a Robot Joint . . . . . . . . . . . . 106

2.1 A procedure to estimate friction at a fixed speed level . . . 107

2.2 A model for the nominal behavior of friction . . . . . . . 109

2.3 A model for the effects of wear to friction . . . . . . . . 112

2.4 A complete model of steady-state friction . . . . . . . . . 114

3 Model-Based Wear Estimation . . . . . . . . . . . . . . 115 
3.1 Maximum likelihood estimation . . . . . . . . . . . . 117

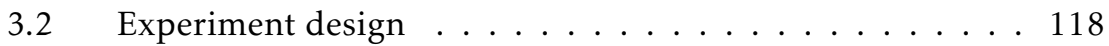

4 Simulation Study . . . . . . . . . . . . . . . . . 119

4.1 Definition of parameters used . . . . . . . . . . . 119

4.2 Experiment design ................ 120

4.3 Bias and variance properties of the wear estimators . . . . 122

5 Studies based on Real Data . . . . . . . . . . . . . . 123

5.1 Description of scenarios . . . . . . . . . . . 124

5.2 Results and discussion .................... 125

6 Conclusions and Future Work . . . . . . . . . . . . . . . 129

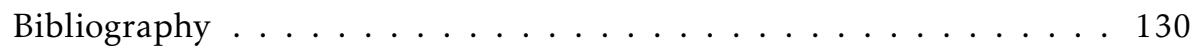

C Data-Driven Diagnostics of Repetitive Processes 133

1 Introduction . . . . . . . . . . . . . . . . 135

2 Data-Driven Diagnostics and Repetitive Systems . . . . . . . . . 137

2.1 Detection, performance and isolation . . . . . . . . . 138

2.2 Repetitive systems . . . . . . . . . . . . . 139

3 A Distribution Domain Approach . . . . . . . . . . . . 140

3.1 Characterizing the data - Kernel Density Estimate . . . . 140

3.2 Comparing sequences - Kullback-Leibler distance . . . . . 142

3.3 Handling non-repetitive disturbances and noise . . . . . . 143

4 Wear Monitoring in an Industrial Robot Joint . . . . . . . . . . . . 145

4.1 Experimental studies under constant disturbances . . . . . 146

4.2 Simulation studies under temperature disturbances . . . . 147

5 Gearbox Monitoring based on Vibration Data . . . . . . . . . . 151

6 Conclusions and Future Work . . . . . . . . . . . . . . 154

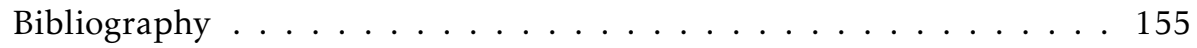

D Simulation based Evaluation of Fault Detection Algorithms 159

1 Introduction . . . . . . . . . . . . . . . . 161

1.1 Problem description and motivation . . . . . . . . . . 162

1.2 Main contributions and outline . . . . . . . . . . 163

2 Design of Experiments . . . . . . . . . . . . . . . . 164

2.1 Choice of input factors . . . . . . . . . . . 164

2.2 Surrogate models as linear regressions . . . . . . . . . 165

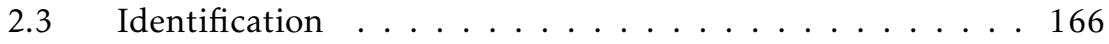

2.4 Design matrix . . . . . . . . . . . . . . 166

2.5 Design parameters .................... 167

2.6 Model validation . . . . . . . . . . . . . 167

3 Determining Relevant Factors . . . . . . . . . . . . . . . 168

3.1 Normalization of coefficients . . . . . . . . . . . 168

3.2 Group analysis . . . . . . . . . . . . 168

4 Comparing Fault Detection Algorithms . . . . . . . . . . . 169

4.1 Two hypotheses . . . . . . . . . . . . . . 169

4.2 A measure of average effects . . . . . . . . . . . 169

4.3 Group analysis . . . . . . . . . . . 170 
5 Determining the Effective Scope . . . . . . . . . . . . . 170

5.1 A measure of satisfactory performance . . . . . . . . 170

5.2 Finding the effective scope . . . . . . . . . . 171

5.3 Group analysis . . . . . . . . . . . . . 171

6 Evaluation of FDAs for Wear Monitoring in Robots . . . . . . . . 172

6.1 Design of experiments ............... 173

6.2 Determining relevant factors . . . . . . . . . 175

6.3 Comparing fault detection algorithms . . . . . . . 176

6.4 Determining the effective scope . . . . . . . . . . 178

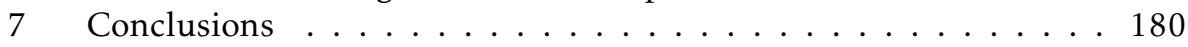

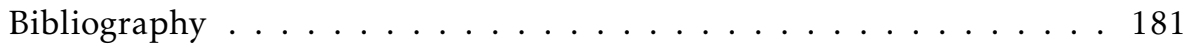

E Data-Driven Anomaly Detection based on a Bias Change 183

1 Introduction . . . . . . . . . . . . . . . . 185

2 The Bias Change Model and the GLR test . . . . . . . . . . . . . 187

2.1 Unknown change time . . . . . . . . . . . . . 188

2.2 Sequential solution . . . . . . . . . . . . . . 189

2.3 Asymptotic performance . . . . . . . . . . . . 189

3 Nonparametric Density Estimators . . . . . . . . . . . . . . 190

3.1 Kernel density estimator . . . . . . . . . . . . . . 190

3.2 A sparse density estimator . . . . . . . . . . . . . 191

4 Estimating the Bias Change . . . . . . . . . . . . . 193

4.1 Batch estimation using EM . . . . . . . . . . 193

4.2 Sequential estimation using stochastic approximation . . . 196

5 Illustrative Examples . . . . . . . . . . . . . . . . . . . . . . 198

5.1 Simulation study . . . . . . . . . . . . . 198

5.2 Batch detection of an increase in eruptions . . . . . . . . 199

5.3 Sequential detection of wear in a robot joint . . . . . . . 199

6 Conclusions and Future Work . . . . . . . . . . . . . . . 203

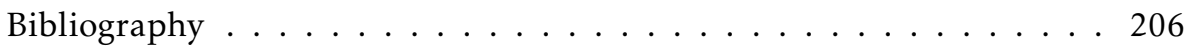


Abbreviations

\begin{tabular}{cl}
\hline Abbreviation & Meaning \\
\hline ISO & International Organization for Standardization. \\
ABB & Asea Brown Boveri Ltd. \\
SRAM & Safety, Reliability, Availability and Maintainability. \\
CBM & Condition Based Maintenance. \\
KLD & Kullback-Leibler Divergence. \\
CRB & Cramér-Rao lower Bound. \\
KDE & Kernel Density Estimate. \\
ROC & Receiver Operating Characteristic. \\
DOF & Degree of Freedom. \\
BL, ML, EHL & Boundary, Mixed and Elasto-Hydrodynamic Lubrica- \\
& tion regions of the friction curve. \\
\hline
\end{tabular}

Basic Mathematical Notation

\begin{tabular}{cl}
\hline Notation & Meaning \\
\hline$x \in \mathbb{X}$ & Scalar quantity from set $\mathbb{X}$. \\
$x \in \mathbb{X}^{n}$ & Column vector of size $n$ with elements in $\mathbb{X}$. \\
$x_{i}$ & The $i$ th element of vector $x$. \\
$X \in \mathbb{X}^{n \times m}$ & Matrix with $n$ rows and $m$ columns with elements in $\mathbb{X}$. \\
$x_{i j}$ & The element of $X$ in the $i$ th row and $j$ th column. \\
{$[X]_{i j}$} & Alternative notation for $x_{i j}$. \\
$X_{i: j}$ & Submatrix of $X$ composed of columns from $i$ to $j$. \\
$X_{j}$ & Shorthand notation for $X_{1: j}$. \\
$f(x): \mathbb{X} \mapsto \mathbb{Y}$ & Scalar function map. \\
$f(x): \mathbb{X} \mapsto \mathbb{Y}^{n}$ & Vector function map.
\end{tabular}




\section{Operators and Special Functions}

\begin{tabular}{cl}
\hline Notation & Meaning \\
\hline$\triangleq$ & Equal by definition. \\
$\sim$ & Denotes "is distributed according to". \\
$\propto$ & Denotes "is proportional to". \\
$|x|$ & Denotes the Hadamard, element-wise, multiplication. \\
$|X|$ & Modulus of $x$. \\
$|\mathbb{X}|$ & The determinant of matrix $X$. \\
$\|\cdot\|_{\delta}$ & The $\delta$ vector or induced matrix norm. \\
$X^{T}$ & The transpose of matrix $X$, i.e., $Y=X^{T}$ implies $y_{i j}=x_{j i}$. \\
$\langle\boldsymbol{x}, y\rangle_{P}$ & Denotes the weighted inner product $x^{T} P y$. \\
$\operatorname{sign}(x)$ & The function satisfying $x=$ sign $(x)|x|$ and sign $(0)=0$. \\
$\arg \min f(x)$ & The value of $x$ that minimizes $f(x)$. \\
$x$ & \\
$\frac{\mathrm{d}}{\mathrm{d} x} f(x)$ & Derivative of $f(x)$ with respect to $x$. \\
$\frac{\partial}{\partial \boldsymbol{x}} f(\boldsymbol{x})$ & Gradient of $f(\boldsymbol{x})$ with respect to $x$. \\
$\dot{x}(t)$ & Derivative of $x(t)$ with respect to time. \\
$\mathbf{T}\{f(x)\}$ & Integral transform of $f(x)$. \\
$\mathbf{F}\{f(x)\}$ & Fourier transform of $f(x)$. \\
$\mathbf{F}^{-1}\{f(v)\}$ & Inverse Fourier transform of $f(v)$. \\
\hline
\end{tabular}

Notation for Probability, Statistics and Decision Theory

\begin{tabular}{cl}
\hline Notation & Meaning \\
\hline$y$ & Sample from the random variable $Y$. \\
$\operatorname{Pr}[Y \in A]$ & Probability of an event $A$. \\
$p(y)$ & Probability dist. (density) function, $\frac{\mathrm{d}}{\mathrm{d} y} \operatorname{Pr}[Y \leq y]$. \\
$\mathbb{E}[f(y)]$ & Expectation of $f(y), \int_{-\infty}^{\infty} f(y) p(y) \mathrm{d} y$. \\
$\Phi(v)$ & Characteristic function, $\mathbb{E}\left[e^{j v y}\right]$. \\
$\mathcal{N}(\mu, \Sigma)$ & The multivariate Gaussian density. \\
$\mathcal{U}(y, \bar{y})$ & The uniform density with limits $\underline{y}$ and $\bar{y}$. \\
$D_{\mathrm{KL}}(p \| q)$ & Kullback-Leibler divergence between $p(y)$ and $q(y)$. \\
$\mathrm{KL}(p, q)$ & Symmetric Kullback-Leibler divergence, $D_{\mathrm{KL}}(p \| q)+$ \\
& $D_{\mathrm{KL}}(q \| p)$. \\
$\mathcal{H}^{0}$ & Null hypothesis in a binary test. \\
$\mathcal{H}^{1}$ & Alternative hypothesis in a binary test. \\
$\phi(q)$ & Decision function in a binary test, $\phi(q): \mathbb{R} \mapsto\{0,1\}$. \\
$\mathcal{R}_{0}$ & Acceptance region in a binary test, $\mathcal{R}_{0} \triangleq\{q: \phi(q)=0\}$. \\
$P_{f}$ & Probability of incorrectly choosing $\mathcal{H}^{1}$ in a binary test. \\
$P_{m}$ & Probability of incorrectly choosing $\mathcal{H}^{0}$ in a binary test. \\
\hline
\end{tabular}




\section{Notation FOR Robotics}

\begin{tabular}{|c|c|}
\hline Notation & Meaning \\
\hline.$a$ & Denotes a quantity described in the arm side. \\
\hline$m$ & Denotes a quantity described in the motor side. \\
\hline${ }^{r}$ & Denotes a reference signal. \\
\hline$\Lambda$ & Inverse gear ratio matrix. \\
\hline$\varphi$ & Vector of joint angular positions. \\
\hline$i$ & Vector of applied motor currents. \\
\hline$\tau$ & Vector of applied torques. \\
\hline$\tau^{f}$ & Vector of joint friction torques. \\
\hline$\tau^{g}$ & Vector of gravity-induced torques. \\
\hline$\tau^{\ell}$ & Component of $\tau^{g}$ parallel to the joint DOF. \\
\hline$\tau^{p}$ & Resulting component of $\tau^{g}$ perpendicular to $\tau^{\ell}$. \\
\hline$\xi$ & Joint lubricant temperature. \\
\hline$\omega$ & Joint wear level. \\
\hline$\mho$ & A trajectory. \\
\hline $\mathcal{J}(\cdot)$ & Analytical Jacobian. \\
\hline $\mathcal{L}(\cdot, \cdot)$ & Lagrangian function. \\
\hline $\mathcal{K}(\cdot, \cdot)$ & Kinetic energy. \\
\hline $\mathcal{P}(\cdot)$ & Potential energy. \\
\hline$M(\cdot)$ & Inertia matrix. \\
\hline$C(\cdot)$ & Matrix of Coriolis and centrifugal torques. \\
\hline$K(\cdot)$ & Stiffness matrix. \\
\hline$D(\cdot)$ & Damping matrix. \\
\hline$p_{i}$ & $i$ th coordinate frame. \\
\hline $\bar{p}_{i}$ & $i$ th homogeneous coordinate frame. \\
\hline$R_{i}^{i-1}$ & Rotation from frame $i$ to $i-1$. \\
\hline$d_{i}^{i-1}$ & Translation from frame $i$ to $i-1$. \\
\hline$H_{i}^{i-1}$ & Homogeneous transformation from frame $i$ to $i-1$. \\
\hline$x$ & End-effector pose (position and orientation). \\
\hline
\end{tabular}




\section{Notation for Friction Modeling}

\begin{tabular}{cl}
\hline Notation & Meaning \\
\hline$f$ & Generalized friction. \\
$\boldsymbol{x}$ & Generalized friction states vector. \\
$h(\cdot)$ & Velocity weakening of the friction curve. \\
$z$ & Velocity strengthening of the friction curve. \\
$\sigma_{0}, \sigma_{1}$ & Internal friction state in a dynamic friction model. \\
$f_{c}$ & Coulomb friction parameter. \\
$f_{s}$ & Standstill (static) friction parameter. \\
$f_{v}$ & Viscous friction parameter. \\
$f_{\mu}$ & Non-Newtonian viscous friction parameter. \\
$\dot{\varphi}_{s}$ & Stribeck speed friction parameter. \\
$\alpha$ & Stribeck speed exponent parameter. \\
$\beta$ & Non-Newtonian viscous friction exponent parameter. \\
\hline
\end{tabular}


Notation for Models of Systems and IDEnTification

\begin{tabular}{|c|c|}
\hline Notation & Meaning \\
\hline $\boldsymbol{u}$ & Control input vector. \\
\hline$y$ & Measured output vector. \\
\hline$r$ & Reference vector. \\
\hline$d$ & Unknown disturbance vector. \\
\hline$f$ & Unknown fault vector. \\
\hline$z$ & Deterministic input vector. \\
\hline$v$ & Random input vector. \\
\hline$k$ & Sample index in $\mathbb{N}$. \\
\hline$N$ & Total number of data samples. \\
\hline$\theta$ & Vector of parameters. \\
\hline$\theta^{0}$ & True vector of parameters. \\
\hline$\widehat{\theta}$ & Estimate of the parameters $\theta^{0}$. \\
\hline$\widehat{\boldsymbol{\theta}}_{N}$ & Parameter estimate achieved from $N$ samples. \\
\hline $\mathcal{M}$ & Model structure. \\
\hline $\mathcal{M}(\theta)$ & A model instance of $\mathcal{M}$ determined by $\theta$. \\
\hline$\phi(\cdot)$ & Regression vector function. \\
\hline$\Phi(\cdot)$ & Matrix of stacked regressors. \\
\hline$\eta$ & Parameters that appear linearly in the regression. \\
\hline$\rho$ & Parameters that appear nonlinearly in the regression. \\
\hline$y_{k}$ & Vector of measurements at index $k$. \\
\hline$\widehat{y}(k \mid \theta)$ & Predictor function at index $k$. \\
\hline$\epsilon(k, \theta)$ & Prediction error function, $y_{k}-\widehat{y}(k \mid \theta)$. \\
\hline$\psi(k, \theta)$ & Gradient $-\frac{\partial}{\partial \theta} \epsilon(k, \theta)$ \\
\hline $\mathcal{L}(\theta)$ & Likelihood function. \\
\hline $\mathcal{F}\left(\theta^{\prime}\right)$ & Fisher information matrix evaluated at $\theta^{\prime}$. \\
\hline
\end{tabular}





\section{1}

\section{Introduction}

Driven by the severe competition in a global market, stricter legislation and increase of consumer concerns towards environment and health/safety, industrial systems are faced with high requirements on safety, reliability, availability, and maintainability (SRAM). In the industry, equipment failure is a major factor of accidents and down time (Khan and Abbasi, 1999; Rao, 1998). While a correct specification and design of the equipments are crucial for increased SRAM (Thompson (1999)), no amount of design effort can prevent deterioration over time and equipments will eventually fail. However, the associated impacts can be considerably reduced by appropriate maintenance practices. Fault diagnosis methods can be used to determine the condition of the equipment, detect and identify faults and are thus desirable to support service. Fault diagnosis can be used to increase SRAM and reduce the overall costs of service, e.g., by allowing for condition-based maintenance (CBM).

This thesis addresses the design of fault diagnosis methods for an equipment which is many times of crucial importance in manufacturing, industrial robots. The main focus is on the monitoring and detection of excessive degradations caused by wear of the mechanical parts. The wear processes may take several years to be of significance, but can evolve rapidly once it starts to appear. An early detection of excessive wear levels can allow for CBM and increased SRAM. Since wear is related to friction, the basic idea pursued is to analyze the behavior of friction in order to infer about wear. To allow this, an extensive study of friction in robot joints is performed and different solutions for detection of wear related changes are proposed and evaluated. This chapter presents an introduction and motivation to the problem, followed by the outline and main research contributions of the thesis. 


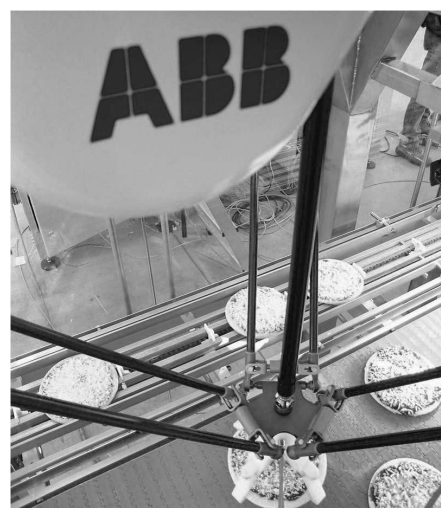

(a) Pick and place.

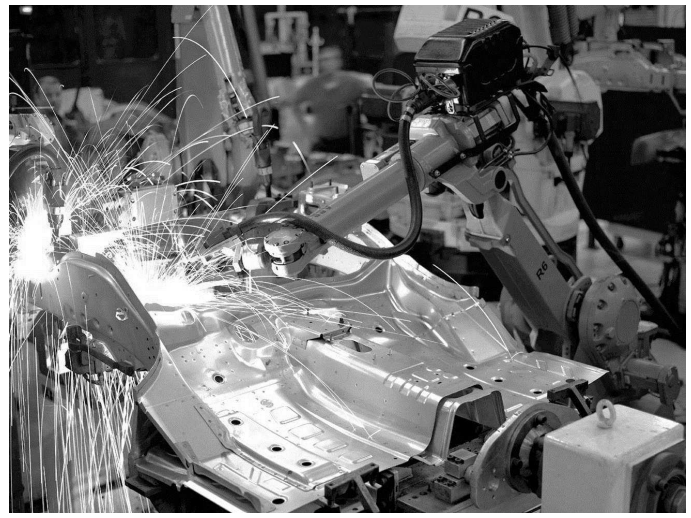

(b) Spot welding.

Figure 1.1: Examples of applications of industrial robots where high availability is critical. The economical damages of an unpredicted robot stop in a production line are counted by the second.

\subsection{Motivation}

Industrial robots are used as a key factor to improve productivity, quality and safety in automated manufacturing. Robot installations are many times of crucial importance in the processes where they are used. As illustrated by the applications found in Figure 1.1, an unexpected robot stop or malfunction has the potential to cause downtimes of entire production lines, with consequent production losses and economical damages. Availability and maintainability are therefore critical for industrial robots. An automated supervision of the robot system is desirable as it relieves operators and can increase SRAM. Collision detection and brake monitoring are examples of functionality available in commercial products that can improve the safety and the integrity of the system. However, there are currently little commercial solutions that allow for an automated monitoring of the mechanical parts of the robot.

For industrial robots, the requirements on high availability are most of the times achieved based on preventive and corrective maintenance policies. Service routines are typically performed on-site, with a service engineer. Service actions are based on specific on-site tests or simply from a pre-determined schedule. The later is scheduled based on the estimated lifespan of components, with considerable margins. Such maintenance solutions can deliver high availability, reducing downtimes. The drawbacks are the high costs due to on-site inspections by an expert and/or due to unnecessary maintenance actions that might take place.

In the current scenario, the serviceability of industrial robots can be greatly improved with the use of methods to infer the system condition and determine imminence of a critical degradation, allowing for СBM. There are however require- 
ments from both the robot user and the service contractor.

The robot user seeks for improved SRAM. Therefore, the solution should be reliable and accurate, with minimal intervention with the operation of the system.

The service contractor seeks for reduced service costs. Therefore, a remote and automated solution, with no extra sensors would be desirable.

Achieving these compromises is a challenging task. This is partly because some faults are difficult to predict, or affect the operation of the system abruptly, e.g., a wire cut or a power supply drop. These types of faults, even when detected, might still cause damages. Therefore, with focus on avoiding failures, the interest is limited to faults that can be diagnosed before a critical degradation takes place, so that timely maintenance actions can be performed.

An important type of such fault is related to the wear processes in a robot joint. Wear develops with time/usage and critical wear levels might be detected at an early stage, allowing for CBM. The wear processes inside a robot joint cause an eventual increase of wear debris in the lubricant. A possible solution is therefore to monitor the iron content in the lubricant. For a typical robot setup, this type of approach will however contradict most of the user's and service contractor's requirements.

An important characteristic of wear is that it affects friction in the robot joint. An alternative solution, explored in this work, is thus to monitor friction changes to infer about wear. Since the friction torques must be overcome by the motor torques during its operation, it is possible to extract information about friction from available signals. Friction is however dependent on other factors than wear. In fact, friction changes caused by, e.g., temperature are typically at least as significant as those caused by wear.

\subsection{Research Goals and Approach}

The main objectives of this work can be explicit as follows.

Design and investigate the applicability of methods to detect critical changes of wear based on standard sensory information and limited intervention with the system operation to support service.

The approach to the problem can be described by the following tasks.

Extensive studies of friction. Because friction and wear are related, the problem is initially approached by an extensive experimental study of friction in robot joints in order to determine how critical changes of wear may affect the system and the available data.

At this stage, it is identified that the effects of wear to friction are comparable to those caused by temperature and load, which are not measured and can considerably vary in practice. To allow for a more extensive evaluation 
of the proposed methods for wear monitoring as well as to be used in their design, a friction model is developed that can describe the effects of speed, load, temperature and wear.

Design of methods for wear monitoring. The developed friction model is used to define an approach to wear monitoring based on the estimation of a wear related quantity. Aiming at increasing the portfolio of possible service offerings, an alternative method for wear monitoring is also suggested that does not require knowledge of a friction model and is only based on available data.

Extensive evaluation of monitoring methods. To verify the applicability of the proposed methods, they are evaluated under realistic scenarios based on real data and extensive simulations. In particular, a framework for simulation based evaluation and comparison of different solutions is proposed which can be used to reveal important properties of the problem at hand and of candidate solutions.

Design of methods for the detection of changes. A tool for an automated determination of fault presence is also devised which can provide an estimate of the decision errors, supporting service decisions at a higher level.

This work is in the overlap of three main research areas, namely: industrial robotics, tribology and fault diagnosis. To consider a problem in their intersection will require understanding of the available techniques from each of these fields. Therefore, much of this thesis is dedicated to provide an overview of these research areas. This will help to motivate the research presented and to identify needs for innovative solutions. The outline of the thesis and the main contributions are described next.

\subsection{Thesis Outline}

The thesis is divided into two parts. Part I gives an overview of the related research areas and provides a background to the research contributions. The research contributions are presented in Part II, which contains edited versions of published papers.

The outline for Part I is summarized below.

Chapter 2 provides an introduction to industrial robotics. The purpose is to provide an overview of important aspects of the application, the main limitations and challenges.

Chapter 3 focuses on describing friction and wear phenomena in industrial robot joints. It provides an overview of the friction and wear processes, and of some of the challenges behind the research goals of this work.

Chapter 4 provides an overview of fault diagnosis. It includes a description of the different tasks in fault diagnosis and the existing compromises in their 
design. Examples are given to provide an overview of different methods for monitoring wear in a robot joint.

Chapter 5 presents a summary of the thesis, conclusions and recommendations to future work.

Each chapter in Part I is concluded by presenting connections to the research papers of Part II. A summary of the main research contributions of Part II is given below.

Extensive studies of friction in a robot joint are presented in Papers A and B. The effects of joint angle, load torques, temperature and wear are analyzed through empirical studies.

Friction modeling, the effects of load torques and temperature to friction in a robot joint are modeled and identified in Paper A.

Wear modeling, the effects of wear to friction in a robot joint are also modeled and identified in Paper B.

Wear identification. In Paper B, a solution for wear monitoring is proposed based on the identification of a wear related quantity from friction data.

Diagnosis of repetitive systems. Data-driven methods suitable for repetitive processes are suggested and verified experimentally in Paper C.

Evaluation of methods for scenarios of practical relevance are presented in $\mathrm{Pa}$ pers B and C. A simulation based framework for the evaluation of fault detection algorithms is also suggested in Paper D in a general setup.

Anomaly detection, in Paper E, data-driven methods are proposed for anomaly detection that only require availability of a nominal dataset and minimal / meaningful specifications from the user. Estimates of the decision uncertainties are also given which can support service decisions at a higher level.

\subsubsection{Background on the publications}

Edited versions of the following papers are included in Part II of this thesis. The background for the research contributions in each paper is discussed next.

\section{Paper A: Friction in a Robot Joint - Modeling and Identification of Load and Temperature Effects}

A. C. Bittencourt and S. Gunnarsson. Static friction in a robot jointModeling and identification of load and temperature effects. Journal of Dynamic Systems, Measurement, and Control, 134(5), July 2012.

Several reports can be found in the literature regarding the dependency of friction in a robot joint to other factors than speed, e.g., Gogoussis and Donath (1988); Waiboer et al. (2005); Hamon et al. (2010). However, to the best of the author's knowledge, no detailed empirical studies of these effects in a robot joint had been previously published. 
This work provides a deeper understanding of these phenomena based on experiments that were carried out during the summer of 2009 at ABB. The main motivation for the studies was to gather understanding of these phenomena. This would serve as a pre-requisite to the development of wear monitoring methods based on analysis of the friction behavior. As a result, a model that can explain the effects of temperature and load to friction was developed and validated. The developed model is important not only for the design and validation of diagnosis methods but also for control and simulation.

\section{Paper B: Modeling and Experiment Design for Identification of Wear in a Robot Joint Under Load and Temperature Uncertainties}
A. C. Bittencourt and P. Axelsson. Modeling and experiment design for identification of wear in a robot joint under load and tempera- ture uncertainties based on friction data. IEEE/ASME Transactions on Mechatronics, 19(5):1694-1706, October 2014.

Different approaches had been previously proposed for monitoring of friction changes based on parameters estimated from a friction model. However, no report could be found that considered the effects of wear changes explicitly. Moreover, no detailed studies of the undesired effects of disturbances caused by temperature and load to friction were found. This is partly because there were no available models to explain these phenomena. Another important aspect is that performing experiments for wear monitoring is a very time consuming and expensive task.

Based on accelerated wear experiments performed in cooperation with ABB, the effects of wear to friction were studied and a model to explain the effects of wear to friction was developed. This model, combined with the model of Paper A, is very important for the design and evaluation of solutions for wear diagnosis and are used extensively through Part II. In this paper, the models are used in the proposed method for the estimation of a wear related quantity. As it is shown, a careful experiment design can lead to reliable estimates of the wear quantity, despite the presence of disturbances and modeling uncertainties.

\section{Paper C: A Data-driven Approach to Diagnostics of Repetitive Processes in the Distribution Domain}
A. C. Bittencourt, K. Saarinen, S. Sander-Tavallaey, S. Gunnarsson, and M. Norrlöf. A data-driven approach to diagnostics of repetitive processes in the distribution domain - Applications to gearbox diag- nostics in industrial robots and rotating machines. Mechatronics, $-(0)$ : -, 2014. available online.

A repetitive operation is found in various applications, e.g., in automated manufacturing. Repetition can also be forced with the execution of specific diagnostic routines but with the drawback of reduced availability. The repetitive execution of a system provides redundancies about the system's behavior which are directly found in the data. For example, it is possible to compare the results of the exe- 
cution of a diagnostics routine performed today to how it is performed in a year. The differences in the results can relate the system's deterioration over the period. The ideas behind the methods emerged via a combination of development and testing of methods in collaboration with ABB.

The methods were developed with the interest focused on diagnosis of industrial robots, where a repetitive operation is commonly found and repetitive data can thus be found during normal operation. As shown in the paper, with little design requirements, the proposed methods can be used to monitor wear changes despite presence of disturbances. Applicability to other types of mechanical systems is also studied based on vibration data.

\section{Paper D: Simulation based Evaluation of Fault Detection Algorithms}
A. Samuelsson, A. C. Bittencourt, K. Saarinen, S. S. Tavallaey, M. Nor- rlöf, H. Andersson, and S. Gunnarsson. Simulation based evaluation of fault detection algorithms with applications to wear diagnosis in manipulators. In Proceedings of the 19th IFAC World Congress, Cape Town, South Africa, 2014.

Before deployment of fault detection solutions, it is important to study the behavior of the methods in practical scenarios. The evaluation of wear monitoring methods based on field or laboratory studies is time and cost critical and the use of simulations is a more viable alternative.

This paper aims at providing a framework for the evaluation and comparison of fault detection algorithms. Simulation based approaches are proposed in an attempt to determine which disturbances affect a given method the most, how to compare different methods and how to determine the combination of disturbances and faults effects where the methods perform satisfactorily.

This work was motivated by the needs at ABB of a framework to evaluate different available methods for wear monitoring and was partly carried out during Andreas Samuelsson's Master thesis,

A. Samuelsson. Simulation based Evaluation of Mechanical Condition Change Methods. MSc. thesis LiTH-ISY-EX-11/4575-SE, Department of Electrical Engineering, Linköping University, Linköping, Sweden, 2012.

\section{Paper E: Data-Driven Anomaly Detection based on a Bias Change}
A. C. Bittencourt and T. Schön. Data-driven anomaly detection based on a bias change. In Proceedings of the 19th IFAC World Congress, Cape Town, South Africa, 2014.

In order to decide for the presence of a critical condition, a decision rule, e.g., a threshold check, is needed. Optimal decision rules are possible that minimize the probabilities of making incorrect decisions, i.e., likelihood ratio tests. The optimal decision rule requires availability of statistical models for the quantity being tested in both normal and abnormal conditions. Often, such statistical 
models are not available, in particular for the abnormal case, and approximations or assumptions are introduced to devise a decision rule.

In this paper, a data-driven method is proposed to find an approximate test that only requires availability of nominal data and specification of a desired error probability. It is based on the assumption that an abnormality will appear as a bias change relative to nominal, which is rather intuitive. The advantages lie in the flexibility of the approach, minimal specification requirements from the user and the possibility to provide estimates of the decision errors.

\subsubsection{Relevant and additional work}

The author was introduced to the wear monitoring problem already in 2007 during a Master Thesis project carried out at ABB,

A. C. Bittencourt. Friction Change Detection in Industrial Robot Arms. MSc. thesis XR-EE-RT 2007:026. Department of Electrical Engineering, The Royal Instute of Technology (KTH), Stockholm, Sweden, 2007.

In the contribution, a method for friction change detection was developed. The basic idea was to monitor the changes found directly on the friction data. A testcycle was required in order to collect friction data, in a similar way as in Paper B. The effects of load, lubricant and temperature were briefly investigated during the work and motivated the more thorough experiments of Paper A.

The methods presented in Paper $\mathrm{C}$ were submitted as part of the patent application,

S. Sander-Tavallaey, K. Saarinen, H. Andersson, and A. C. Bittencourt.

Condition monitoring of an industrial robot, October 2012. URL http: / /

patentscope.wipo.int/search/en/WO2013050314.

Another patent application during the period of this work is,

A. Isaksson, A. C. Bittencourt, K. Forsman, and D. Peretzki. Method for controlling an industrial process, October 2010. URL http:// patentscope.wipo.int/search/en/WO2012048734,

which describes a method to mine historical process data that can be potentially used to identify models of dynamic systems. The method is described in the paper,

D. Peretzki, A. J. Isaksson, A. C. Bittencourt, and K. Forsman. Data mining of historic data for process identification. In Proceedings of the 2011 AIChE Annual Meeting, October 2011.

This work was not included in the thesis for consistency of the presentation. 
Part I

Background 



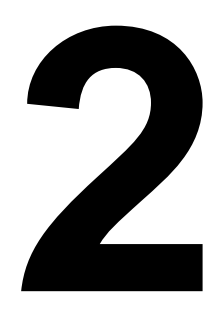

\section{Basics of Industrial Robotics}

The International Organization for Standardization, ISO, proposes the following definitions in ISO 8373 (1994).

Definition 2.1 (ISO 8373:1994 No. 2.15 - Robotics).

Robotics is the practice of designing, building and applying robots.

Definition 2.2 (ISO 8373:1994 No. 2.6 - Manipulating industrial robot). A manipulating industrial robot is an automatically controlled, reprogrammable, multipurpose, manipulator programmable in three or more axes, which may be either fixed in place or mobile for use in industrial automation applications.

Note: The robot includes the manipulator (including actuators) and the control system (hardware and software).

The above definitions make a clear distinction of industrial robots in the manner that they are used, i.e. "in industrial automation applications". The first industrial robot was operating in 1961 in a General Motors automobile factory in New Jersey. It was Devol and Engelberger's UNIMATE. It performed spot welding and extracted die castings (Westerlund, 2000). Since then, many new applications of industrial robots have been introduced, e.g. welding, cutting, forging, painting, assembling, etc. Industrial robots penetrated quite rapidly in manufacturing and specially in the automotive industry, which is still the largest consumer of industrial robots. In 2007, there were more than one million industrial robots in operation worldwide, reaching around 1.5 million in 2013 and with expected increase rates for the next years (Tencer, 2013). 


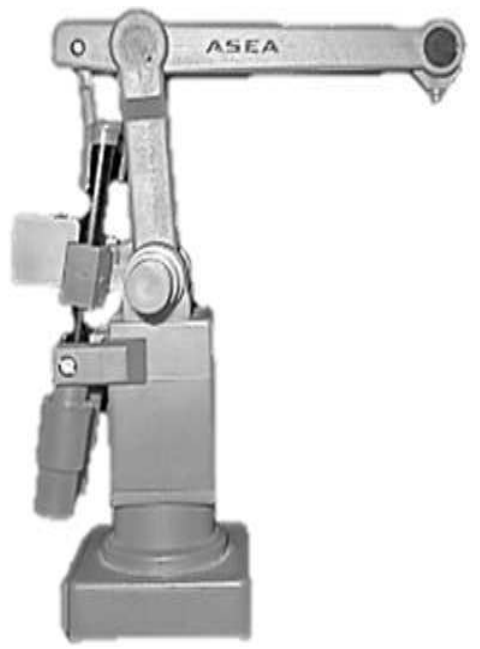

(a) An ABB IRB 6 from 1973.

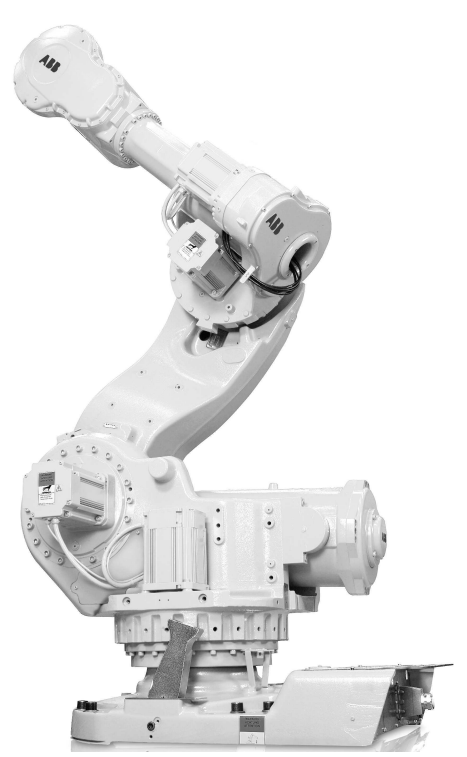

(b) A modern ABB IRB 7600.

Figure 2.1: The fives axes robot IRB 6 was the first all-electrically actuated robot controlled by a microcomputer. The six axes robot IRB 7600 is suitable for high payload applications.

Industrial robots are a key factor to improve productivity, flexibility, quality and safety of technical systems. The history of industrial robotics development is filled with technological milestones. In 1971, the first all-electrically actuated robot was introduced by Cincinnati Millacron, whose robotics development team was later acquired by ABB in 1990. In 1973, ABB released IRB 6, the first microcomputer-controlled robot, which was also all-electrically actuated. Remarkably, this setting is still dominant for modern industrial robots, see Figure 2.1.

The mechanical structure of a standard industrial robot is composed by links and joints. Links are the main bodies that make up the mechanism and the links are connected by joints to each other. A joint constraints the relative motion of the connecting links and are categorized accordingly. The configuration of links and joints defines the kinematic chain of the robot. The number of joints defines the number of manipulated degrees of freedom, DOF, of a robot. The most common configuration of industrial robots is the six DOF with serial kinematics and revolute joints, meaning that links are connected in series through joints allowing for rotational movements. This type of robots are also known as "elbow" manipulators for their resemblance with the upper arm of a human. For elbow manipulators, the first three axes, also called main axes, are used to achieve a desired position of the end-effector. The links of the main axes are bigger since 
they drive more load compared to the last three, wrist axes, which are used to manipulate the orientation of the end-effector.

The main developments in industrial robotics have been directly connected to its main market, the automotive industry. This resulted in products with high cost efficiency, reliability and performance (Brogårdh, 2007). A cost-driven development means the need of cost reduction of the components. This leads to a more difficult control design to handle the larger variations in kinematic and dynamic parameters, lower mechanical resonance frequencies and larger nonlinearities. In order to meet the performance required from industrial robots, a broad understanding of the system is needed. This chapter reviews the basics of industrial robotics.

\subsection{Actuators and Sensors}

An industrial robot is a complete system that interacts with its surroundings. Its degree of autonomy is directly related to the sensory information available, the knowledge built in the system (e.g. models/learning), and the possibilities to perform actions. Following demands on cost efficiency and reliability, the amount and variety of sensors are remarkably small in typical applications of industrial robots. With the development of new applications and higher demands on autonomy, alternative sensors are becoming more common (Brogårdh (2009)).

\subsubsection{Basic setup}

As mentioned in the beginning of this chapter, modern industrial robots are most commonly actuated with electrical motors. The permanent magnet synchronous motor, PMSM, is a popular choice due to its high power density, easy operation and performance. The output torque of such motor can be divided into two parts,

- the dominant electromagnetic torque, arising from the interaction between the rotating magnetic field and the magnet and,

- the pulsating torque, an angular dependent component composed of cogging and ripple torques (Jahns and Soong, 1996).

The pulsating torque leads to challenges in control of machines actuated with PMSM, see, e.g., Proca et al. (2003); Mohamed and El-Saadany (2008). Furthermore, the relation between applied current and output torque varies with temperature due to a reversible demagnetization of the magnets (Sebastian (1995)). A power amplifier is used to modulate the power used as input to the motors.

In order to provide high torques and low speeds, a gearbox transmission is used at the motor output. The rotary vector (RV) type is a popular choice of compact gearboxes due to their low backlash, high gear ratio (in the order of $100-300$ ) and size. This type of transmission is commonly found in the main axes of a manipulator. In the wrist axes, also harmonic drive gears are used as well as special gear solutions. See Figure 2.2 for examples of motor and gear units used in industrial robots. 

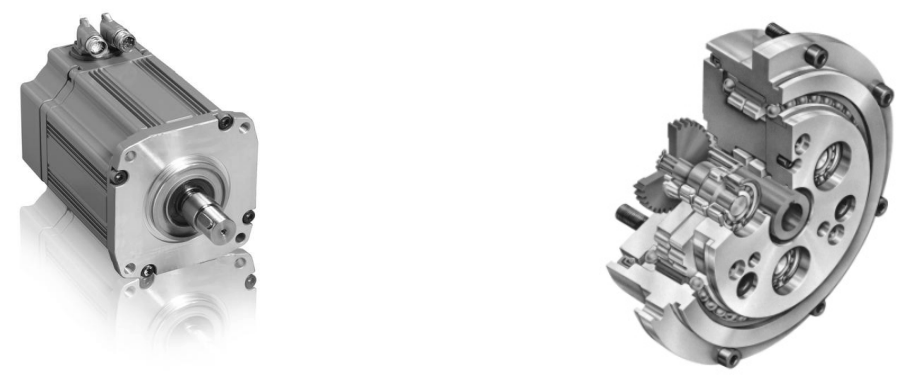

Figure 2.2: An ABB motor (left) and a Nabtesco RV gear unit scheme (right, picture courtesy of Nabtesco.)

Typically, only the rotation angle of the motor shaft, electrical quantities (voltages and currents), and winding temperature are measured. Optical encoders and resolvers are the most commonly used sensors for the angular measurements. The high accuracy of encoders and resolvers used allows for differentiation of the angular measurements to provide estimates of speed and acceleration.

\subsubsection{Application dependent sensors}

With the basic sensors and refined models of the system, it is possible to achieve high path and positioning performances. This allows robots to be used in applications with a controlled/predictable environment. In more demanding applications, where the workpiece and environment are changing or in contact applications, the use of alternative sensors may be needed.

Six DOF force/torque sensors can be used in applications such as high precision assembly of drive trains. This type of sensor is also important in machining applications, such as grinding and polishing, see e.g. Jonsson et al. (2013). The use of high speed cameras combined with image processing algorithms is also important in pick and place applications. Applications demanding very high accuracy might require the use of additional sensors on the arm side of the robot. Measurements of the arm variables help to reduce the influence of backlash and compliance of the gears on the accuracy of the robot. This can be achieved, e.g., with the use of encoders, torque sensors and inertial measurement units, IMU's, in the actuator transmissions and the arm system. For a review, see Brogårdh (2009); for an example on the use of IMU's to improve accuracy, see Axelsson (2014).

Remark 2.1. While the use of additional sensors can increase the robot autonomy, performance and safety, it also means higher costs. 


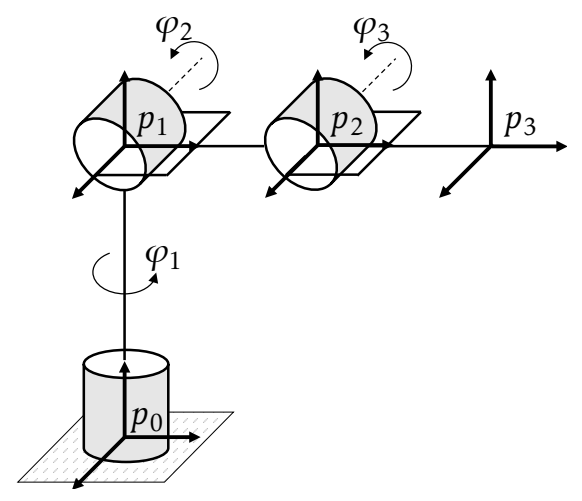

Figure 2.3: Joint positions, $\varphi_{i}$, and coordinate frames, $p_{i-1}$, for an elbow manipulator with joints $i \in\{1,2,3\}$. The end-effector is fixed at frame $p_{3}$.

\subsection{Modeling}

Given the limited sensory information from the measurements of the angles of the motor shafts, the high demands on accuracy and performance expected from industrial robots are only possible with the use of reliable models and modelbased control (Brogårdh (2009)). Models are also important for design, simulation, diagnosis, etc. They play a significant role in all industrial robotics.

In this section, modeling of industrial manipulators is reviewed. The presentation follows standard textbooks, see e.g. Sciavicco and Siciliano (2000) and Spong et al. (2006).

\subsubsection{Kinematics}

The kinematics describes the motion without considering the forces and torques causing it. A kinematic model only depends on the geometric description of the robot. Let $\varphi_{i}$ be the $i$ th joint position at the arm side and $p_{i-1}$ be a frame defined at that joint. For a configuration with $n$ joints, there are $n+1$ frames where the end-effector is considered fixed at frame $p_{n}$. See Figure 2.3 for an illustration.

By using a coordinate transformation, it is possible to describe a point attached to coordinate frame $i$ in the coordinate frame $i-1$ by

$$
p_{i-1}=R_{i}^{i-1} p_{i}+d_{i}^{i-1}
$$

where $R_{i}^{i-1}$ and $d_{i}^{i-1}$ are a rotation and a translation from frame $i$ to frame $i-1$ respectively. The above transformation can be written as a homogeneous transformation

$$
\bar{p}_{i-1} \triangleq\left[\begin{array}{c}
p_{i-1} \\
1
\end{array}\right]=\underbrace{\left[\begin{array}{cc}
R_{i}^{i-1} & d_{i}^{i-1} \\
0 & 1
\end{array}\right]}_{\triangleq H_{i}^{i-1}} \bar{p}_{i},
$$


which facilitates calculations since consecutive frame transformations simplify to multiplications of matrices. Notice that the homogeneous transformation $H_{i}^{i-1}$ is a function of $\varphi_{i}$ and of the links' geometry.

\section{Forward kinematics}

The forward kinematics is the problem of finding the end-effector pose $x$ (position and orientation) relative to the base frame given the joint variables $\varphi$. This can be achieved with the use of a homogeneous transformation from the tool pose to the base frame. For a configuration with $n$ joints, the transformation is described as

$$
H_{n}^{0}(\varphi)=\left[\begin{array}{cc}
R_{n}^{0}(\varphi) & d_{n}^{0}(\varphi) \\
0 & 1
\end{array}\right]
$$

from which it is possible to extract the pose, $x$, of the end-effector. The DenavitHartenberg convention provides a manner to choose the reference frames that allows for a systematic analysis. For a serial robot, the direct kinematics always has a unique solution.

Taking the time derivative of the end effector pose, gives a relation between the joint velocities $\dot{\varphi}$ and the linear and angular velocities of the end-effector as

$$
\dot{x}=\mathcal{J}(\varphi) \dot{\varphi},
$$

where $\mathcal{J}(\varphi)$ is known as the analytical Jacobian matrix. The accelerations can be found by taking the time derivative again, yielding

$$
\ddot{x}=\mathcal{J}(\varphi) \ddot{\varphi}+\left(\frac{\mathrm{d}}{\mathrm{d} t} \mathcal{J}(\varphi)\right) \dot{\varphi} .
$$

The Jacobian matrix is an important quantity in robotics, it can be used to find singular configurations, transformation of tool forces to joint torques, etc.

\section{Inverse kinematics}

The reverse problem, finding the joint positions $\varphi$ given the end-effector pose is known as the inverse kinematics. The inverse kinematics problem is important for trajectory generation, when a desired tool path needs to be transformed to joint positions. For the serial robot, it can be expressed as solving the nonlinear equations

$$
H_{n}^{0}(\varphi)=H_{1}^{0}\left(\varphi_{1}\right) H_{2}^{1}\left(\varphi_{2}\right) \cdots H_{n}^{n-1}\left(\varphi_{n}\right)=H
$$

for a given right-hand side $H$, where $\varphi_{i}$ is $i$ th joint position and $H_{i}^{i-1}$ is given by (2.2). An analytical solution is not always possible, in which case a numerical solver can be used, and even if a solution exists it is typically not unique.

\subsubsection{Dynamics}

A dynamic model describes the relation between the robot motion and the forces and torques that cause it. Dynamic models are important for simulation, trajectory generation and control. In feed-forward control, the motor torques required 
to achieve a certain path are computed from the inverse dynamics.

The simplest modeling approach is to consider all links as rigid bodies. From this simplification, there are different possible methods to derive rigid multi-body models. The Euler-Lagrange formulation considers the Lagragian equation

$$
\mathcal{L}(\varphi, \dot{\varphi})=\mathcal{K}(\varphi, \dot{\varphi})-\mathcal{P}(\varphi),
$$

where the Lagrangian $\mathcal{L}(\varphi, \dot{\varphi})$ is defined as the difference between kinetic, $\mathcal{K}(\varphi, \dot{\varphi})$, and potential energies, $\mathcal{P}(\varphi)$. The equations of motion are given from the EulerLagrange equations

$$
\frac{\mathrm{d}}{\mathrm{d} t} \frac{\partial}{\partial \dot{\varphi}_{i}} \mathcal{L}(\varphi, \dot{\varphi})-\frac{\partial}{\partial \varphi_{i}} \mathcal{L}(\varphi, \dot{\varphi})=\tau_{i}, \quad \text { for } i=1, \ldots, n
$$

where $\tau_{i}$ is the applied torque at joint $i$. By writing the kinetic energy as a quadratic function $\mathcal{K}(\varphi, \dot{\varphi})=\frac{1}{2} \dot{\varphi}^{T} M(\varphi) \dot{\varphi}$, where $M(\varphi)$ is the total inertia matrix, gathering gravitational terms of the form $\tau_{i}^{g}(\varphi)=\frac{\partial}{\partial \varphi_{i}} \mathcal{P}(\varphi)$ into the vector $\tau^{g}(\varphi)$ and terms involving $\dot{\varphi}_{i}^{2}$ and cross-products of $\dot{\varphi}_{i} \dot{\varphi}_{j}$ in $C(\varphi, \dot{\varphi})$, the resulting rigid multi-body model is of the form

$$
M(\varphi) \ddot{\varphi}+C(\varphi, \dot{\varphi}) \dot{\varphi}+\tau^{g}(\varphi)=\tau
$$

where $\tau$ is the vector of applied torques. This model can be extended by including a dissipative friction term, $\boldsymbol{\tau}^{f}$, which is typically modeled as a nonlinear function of $\dot{\varphi}$, see Chapter 3 for more on friction.

\section{Including flexibilities}

In most cases when modeling robots, a rigid multi-body model is not sufficient to describe the system in a realistic manner. The approximation of a rigid gearbox is specially unrealistic for compact gearboxes. Also, with a trend of lighter robots, the flexibilities of bearings- and links are also becoming significant. The model for a flexible robot structure can, as a first approximation, be described by lumped masses connected by springs and dampers.

For instance, a flexible joint model can be achieved by modeling the joint as a system with two masses connected by a torsional spring-damper, as shown in Figure 2.4. Neglecting possible inertial couplings between motor and $\operatorname{arm}^{\mathrm{i}}$, the resulting model can be described as

$$
\begin{aligned}
\tau^{a} & =M^{a}\left(\varphi^{a}\right) \ddot{\varphi}^{a}+C\left(\varphi^{a}, \dot{\varphi}^{a}\right)+\tau^{g}\left(\varphi^{a}\right)+\tau^{f, a}\left(\dot{\varphi}^{a}\right) \\
\tau^{a} & =K\left(\Lambda \varphi^{m}-\varphi^{a}\right)+D\left(\Lambda \dot{\varphi}^{m}-\dot{\varphi}^{a}\right) \\
\tau^{m}-\Lambda \tau^{a} & =M^{m} \ddot{\varphi}^{m}+\tau^{f, m}\left(\dot{\varphi}^{m}\right)
\end{aligned}
$$

where the superscripts ${ }^{a}$ and ${ }^{m}$ relate to variables at the arm and motor sides respectively, $\Lambda$ is the inverse gear ratio matrix, $K$ and $D$ are the stiffness and damping matrices. The friction torque is here divided between the motor and arm side, $\boldsymbol{\tau}^{f, m}\left(\dot{\boldsymbol{\varphi}}^{m}\right)$ and $\boldsymbol{\tau}^{f, a}\left(\dot{\boldsymbol{\varphi}}^{a}\right)$ respectively. Friction occurs at different compo-

\footnotetext{
${ }^{\mathrm{i}}$ According to Spong (1987) this is a reasonable approximation if the transmission ratio is large.
} 
nents in the gearbox, at different gear ratios, meaning different reductions when seen at the motor side. See, e.g. Moberg (2010), for a detailed treatment on modeling of flexible robots.

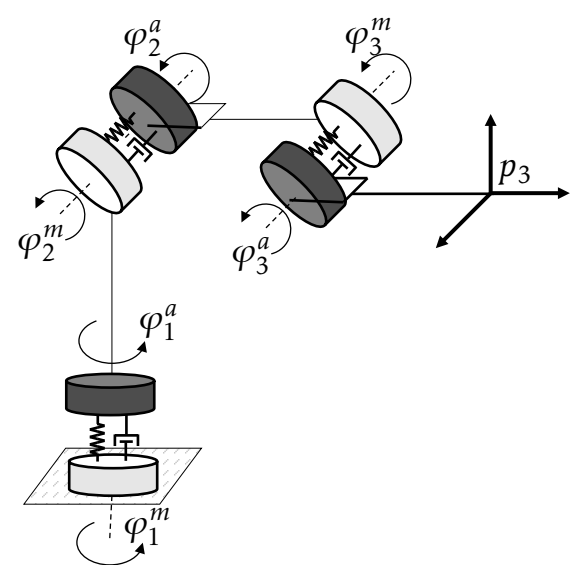

Figure 2.4: Illustration of a flexible robot structure where the flexibilities are modeled as lumped masses connected by springs and dampers.

\subsection{Identification}

The described models depend on a number of parameters that are most often unknown or partly known. In order to make use of models, e.g. for control and simulation, the modeling process can be complemented with identification procedures. Identification is used to find and verify the parametric description of the models from experiments. As introduced in the previous section, the different models can relate to kinematics, dynamics and joint-related phenomena. A summary of these identification problems is given below.

Kinematic models are important for positioning of the end-effector. The parameters in the model relate to the geometric description of the kinematic chain. These parameters can be partly obtained during the design process, e.g. available from CAD models. There are however errors that could relate, amongst other sources, to tolerances during production and assembly of the robot. An identification procedure can be used to correct for these errors, considerably improving the volumetric accuracy of the robot. The process of identifying these parameters is also known as kinematic calibration or robot calibration, and requires measurements of the end-effector position. For a survey on the topic, see Hollerbach (1989).

Dynamic models are important for simulation and feed-forward motion control of robots. The identification of dynamic models of robots is a much studied problem and several approaches can be found, see Wu et al. (2010) for an overview. An important consideration is the type of dynamic model considered. Rigid multi-body models are typically parametrized as a function which is linear in 
the parameters. For example, the model in (2.9) can be rewritten as a linear regression

$$
\tau=\Phi(\varphi, \dot{\varphi}, \ddot{\varphi}) \theta
$$

where $\Phi(\cdot)$ is a matrix regressor function, dependent on $\varphi$ and its derivatives, and $\theta$ are the rigid-body parameters. Based on data from an identification experiment, the parameters $\theta$ can be found, e.g., based on a weighted least squares minimization

$$
\widehat{\boldsymbol{\theta}}=\arg \min _{\boldsymbol{\theta}}(\overline{\boldsymbol{\tau}}-\bar{\Phi} \boldsymbol{\theta})^{T} W(\overline{\boldsymbol{\tau}}-\bar{\Phi} \boldsymbol{\theta})=\left(\bar{\Phi}^{T} W \bar{\Phi}\right)^{-1} \bar{\Phi}^{T} W \overline{\boldsymbol{\tau}},
$$

where $\overline{\boldsymbol{\tau}}$ and $\bar{\Phi}$ are the stacked torque and regressors achieved from the identification experiment. The choice of weight matrix $W$ will affect the solution and different criteria are possible, see, e.g., Gautier and Poignet (2001); Swevers et al. (1997). Finally, the trajectory must be chosen carefully to avoid excitation of flexible modes and improve the estimation performance. Identification of parameters describing the flexibilities is a more involving problem since only a subset of the states can be measured and a linear regression cannot be formed. These models are however important for improved performance of robot control. For a detailed treatment on identification of dynamic models and flexibilities, see Wernholt (2007); Moberg (2010); Wernholt and Moberg (2011).

Joint models. Due to the complex construction of a robot joint, its characteristics are often uncertain and nonlinear phenomena are common. Nonlinearities that can be of significant influence in a robot joint are related to friction, backlash and nonlinear stiffness. Available parametric models are often achieved from empirical modeling for a specific platform since it is difficult to predict the characteristics of these nonlinearities in general. For example, the amount of backlash and friction will depend on how the joints were assembled. Therefore, these models are most often found from an experimental identification procedure. It is important to notice that the identification of dynamic models is facilitated if an accurate joint model is available. For example, in Wernholt (2007) it is reported that the friction at low speeds makes it difficult to identify the resonances related to a flexibility. This is because friction adds damping to the system. With a known friction model, its effects can be analytically removed from the data, making the identification of dynamic parameters more reliable.

\subsection{Reference Generation and Control}

From the perspective of a robot user, it is convenient to be able to program the robot in a high level of abstraction. Typically, objectives can be defined in the task space, and the user does not need to worry about how each joint is controlled. A robot manufacturer dependent programming language is used where instructions to the robot can be given in task (or joint) space. This can be done manually by typing the code or in some cases by demonstration. This process can also be partly automated with the use of CAD/CAM softwares allowing greater flexibility. 
An example of a robot task program is given in Algorithm 1. In order to perform a task, different problems must be solved.

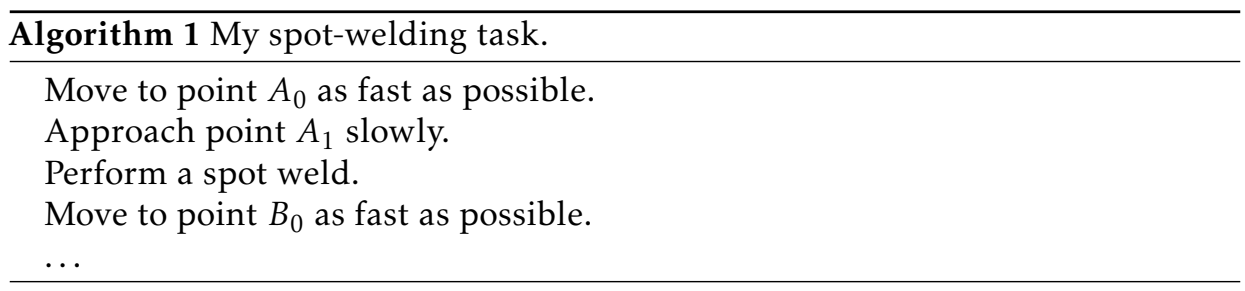

Motion planing. First, given a task, e.g. the one defined in Algorithm 1, a path to be executed by the robot must be generated. This is made by a motion planner, which calculates the movements that the robot must make. At first, the programmed movements are interpreted with respect to what geometry that the path will have (line, circle, spline etc.) and then the path is interpolated to consist of discrete steps, which are transformed from task space to joint space using the inverse kinematic model.

Trajectory generation. The time dependence of the robot movements, i.e. a trajectory, can be calculated either in the task space or in the joint space. Finding a trajectory involves optimization of the use of the dynamic capabilities of the robot with respect to speed- and acceleration performance. Let $\mho \checkmark$ denote a trajectory, the trajectory generation is essentially an optimization problem including,

$$
\begin{aligned}
\mho^{r}=\arg \min _{\mho} & \text { Objective }(\mho) \\
\text { subject to } & \operatorname{Path}(\mho) \\
& \operatorname{Dynamics}(\mho) \\
& \text { Mechanical limitations }(\mho)
\end{aligned}
$$

where the solution, $\mho^{r}$, is used in the next stage as a reference for the motion control. The objective can be, e.g., minimal cycle-time or minimal energy. The constraints ensure that the trajectory runs through the path according to the dynamics of the manipulator and avoiding mechanical limitations such as motor position and speed ranges, maximum allowed forces and torques in the joints, etc. Notice that the solution for this optimization problem can considerably affect the time and performance of the task execution and is highly dependent on the models used. For example, in Ardeshiri et al. (2011) the inclusion of speed dependent constraints in a convex formulation of the problem allowed for reductions of the path tracking time by 5-20\%. Speed-dependent constraints are motivated from physical modeling of the motors and the drive system, they can, e.g., relate to viscous friction.

Motion Control. Finally, when the reference trajectory is generated, it is possible to execute the task with the help of the servo control. Important features of the servo are trajectory tracking, robustness and disturbance rejection. Different control strategies and structures are possible depending on the sensors available, 


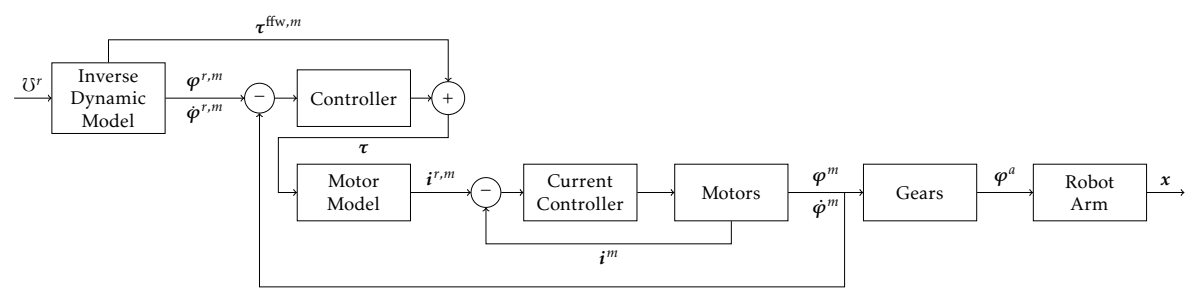

Figure 2.5: A model-based control scheme for trajectory tracking. A feedforward action $\tau^{\mathrm{ffw}, m}$ and motor references $\varphi^{r, m}, \dot{\varphi}^{r, m}$ for the outer feedback loop are computed based on the reference trajectory $\mho^{r}$ using an inverse $d y$ namic model. An inner control loop is used to control the motor current according to $i^{r, m}$ which is achieved from a desired input torque vector $\tau$ using a motor model.

controlled variables, etc., see Moberg (2010); Brogårdh (2009) and available textbooks for details. Here, a common control approach is discussed for the typical setup, with measurements only at the motor side.

\section{Model-based control for trajectory tracking}

An overview of one possible robot control scheme is given in Figure 2.5. The desired trajectory $\mho^{r}$ contains the joint information through time at the arm side, that is, $\varphi^{r, a}$ and its derivatives. With angular position measurements available at the motor side, $\varphi^{m}$, and an estimate of $\dot{\varphi}^{m}$ achieved from differentiation, the arm side references are transformed to the motor side, yielding $\varphi^{r, m}, \dot{\varphi}^{r, m}$ which are used in the outer feedback control loop.

To improve performance, an inverse dynamic model is used to generate feedforward motor torques, $\tau^{\mathrm{ffw}, m}$. The input torque vector $\tau$ is the total torque the motor should generate to drive the robot in the desired manner and is composed of both feed-forward and feedback actions. Since the motor torque is not measured, a motor model is used to transform $\boldsymbol{\tau}$ to a current reference, $\boldsymbol{i}^{r, m}$, for the inner current control loop. The motor variables $\varphi^{m}$ and $\dot{\varphi}^{m}$ are fed back to the outer control loop. At the output is the end-effector pose $\boldsymbol{x}$.

The inner current control loop has much faster dynamics than the outer loop. When designing the outer loop, it is therefore common to accept a constant relation between the measured motor currents and the motor torques, that is $\boldsymbol{\tau}=K \boldsymbol{i}^{m}$. As pointed out in Section 2.1.1, this relation actually varies with temperature since the nominal performance of the motors degrades with increased temperature. 


\subsection{Summary and Connections}

This chapter provided an overview of important aspects to consider when working with industrial robots, the problems, technologies and limitations. Two aspects are particular about the development of industrial robots, the limited sensory information available and the importance of using different types of robot models. The purpose of this introduction has also been to provide a background to the research results presented in the second part of this thesis.

In Papers A and B, an experimental procedure is described for the estimation of constant-speed friction levels in a robot joint. During the procedure, the torque reference to the inner current control loop, recall Figure 2.5, is used as an estimate of the actual applied torques. The approximation of an ideal current loop, giving $\boldsymbol{\tau}=K \boldsymbol{i}^{m}$, is thus important. The gain $K$ should not be speed dependent and the temperature dependence of $K$ should be small. Based on an experimental investigation of the phenomenon for motors of similar types as the ones considered in thesis, Tenerz (2011) shows that variations caused by temperature may be as large as $5 \%$. These dependencies on temperature are therefore neglected during the studies presented in this thesis. 


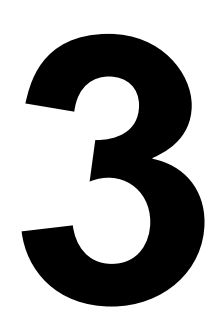

\section{Joint Friction and Wear}

Friction exists in all mechanisms to some extent. It can be defined as the tangential reaction force between two surfaces in contact. There are different types of friction, e.g. dry friction, viscous friction, lubricated friction, skin friction, internal friction. Friction is not a fundamental force but the result of complex interactions between contacting surfaces in down to a nanoscale perspective. Due to its complex nature, it is often difficult to described it from physical principles.

One reason for the interest in friction in the joints of a manipulator is the need to model friction for control purposes. A precise friction model can considerably improve the overall performance of a manipulator with respect to accuracy and control stability, see e.g., Olsson et al. (1998); Bona and Indri (2005); Guo et al. (2008); Susanto et al. (2008); Kim et al. (2009). Since friction can relate to the wear processes of mechanical systems (Blau, 2009), including robot joints, there is also interest in friction modeling for fault detection, see, e.g., Freyermuth (1991); Vemuri and Polycarpou (2004); McIntyre et al. (2005); Mattone and Luca (2009); Brambilla et al. (2008); Caccavale et al. (2009); Namvar and Aghili (2009).

In a robot joint, with several components interacting such as gears, bearings, and shafts, which are rotating/sliding at different velocities and under different lubrication levels, it is difficult to separate and model friction at a component level. A typical approach is to consider these effects collectively, as a "lumped" joint friction. For examples of friction models at a component level, see SKF (2011).

Friction opposes motion, dissipating energy. A part of the work produced by friction appears as heat transfer, vibrations and acoustic emissions. Other outcomes of friction are plastic deformation, adhesion and fracture, see e.g. Bryant (2009). The latter outcomes can relate to wear, which is defined as "the progressive loss of material from the operating surface of a body occurring as a result of relative mo- 

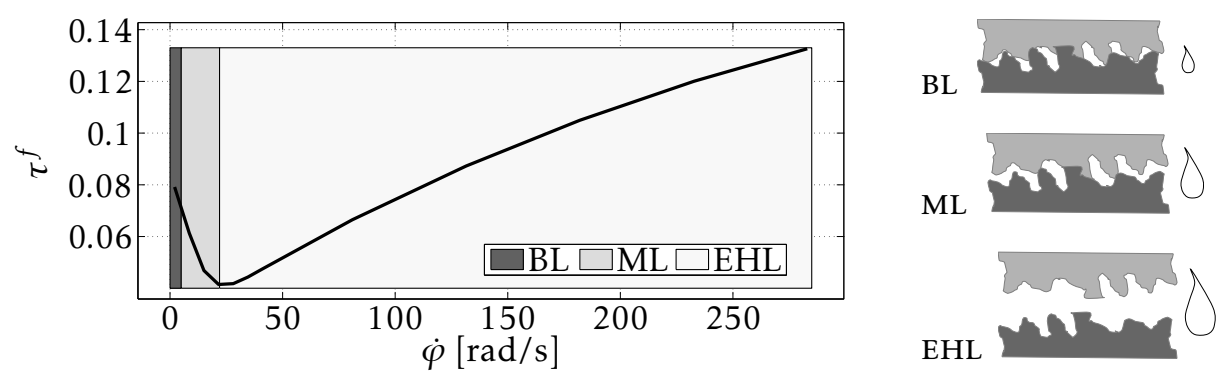

Figure 3.1: Friction curve for constant speed movements and the lubrication regimes illustrated at contact level.

tion at its surface" (Lansdown et al., 1987). The need for relative motion between surfaces implies that wear is related to the mechanical action between surfaces. This is an important distinction to other processes with a similar outcome and very different nature, e.g. corrosion and cavitation.

\subsection{Basics of Tribology}

Tribology is the study of the phenomena taking part in the interaction of surfaces in relative motion, including friction, wear and lubrication. The most important friction characteristics for control applications are usually described by a so-called friction curve, which is a plot of friction levels as function of speed ${ }^{\mathrm{i}}$. An example of such plot achieved from experiments in a robot joint can be seen in Figure 3.1 ii,iii. The nonlinear behavior from low to high speeds is typical in lubricated friction and is known as the Stribeck effect (Woydt and Wäsche, 2010; Jacobson, 2003). This behavior is present in a robot joint due to the presence of lubricant in the gearbox and motor shaft. Notice that the friction in the motor is dry. The use of lubricant is essential to decrease the wear processes. It acts as a separation layer between the surfaces. With the use of additives, e.g. Extreme Pressure (EP) additives, it can even create a chemical barrier between the surfaces under high contact pressures, reducing low speed friction and wear.

The friction curve is divided in three regions according to the lubrication regime: boundary lubrication (BL), mixed lubrication (ML) and elasto-hydrodynamic lubrication (EHL). The phenomenon present at very low speeds (BL) is mostly related to interactions between the asperities of the surfaces in contact. With the

\footnotetext{
${ }^{\mathrm{i}}$ As presented originally by Stribeck (1902), a friction curve is plotted as a function of speed normalized by the ratio of normal load and lubricant viscosity. For simplicity however, it is many times shown only as a function of speed.

${ }^{i i}$ In the figure, the friction torques are normalized to the maximum allowed torque to the joint and are displayed as dimensionless quantities, this convention is followed in the whole thesis.

${ }^{\text {iii }}$ This type of curve is obtained when the speed levels are stable and include no transient phenomena. There are also dynamic effects related to friction, see Section 3.3.
} 
increase of velocity, there is a consequent increase of the lubricant layer between the surfaces with a decrease of contact friction (ML). The decrease of contact friction continues until it reaches a full lubrication profile (EHL), with a separation of the surfaces by the lubricant. In EHL, friction is proportional to the force needed to shear the lubrication layer, and it is thus dependent on the lubricant properties (e.g. viscosity).

The wear processes are most significant in $\mathrm{BL}$ and $\mathrm{ML}$, where contact friction is significant. In a full-film lubrication, there is theoretically no wear taking place, but it still happens because of eventual breakdowns of this layer. It is important to notice that due to the high gear ratio of the gearboxes used in industrial robots, the components closer to the output will be moving slower in comparison to the ones closer to the input. Therefore, at a component level, wear might occur even in the EHL region of the joint friction curve.

\subsection{Friction Dependencies in Robot joints}

At a contact level, friction is dependent on the contact geometry, topology, properties of the materials, position, relative velocity, force/torque levels, temperature, lubricant, etc. (Al-Bender and Swevers, 2008). Depending on the setup, each of these factors will be more or less significant to the total friction.

In robot joints, the friction dependencies will differ depending on the size and type of joint considered. For elbow manipulators, the main axes undertake significant load levels and the wear processes in these axes are usually more significant than in the wrist axes. This thesis focuses on the study of friction and wear in the main axes of large robots, equipped with RV gearboxes, recall Section 2.1.1. The dependencies of friction for such joints have been studied based on experiments, the effects of the most relevant variables to the friction curve are shown in Figure 3.2. It should be noted that, except for Figures 3.2a and 3.2b, the curves are obtained for different robots. The effects are summarized below.

Load. The effects of load follow from the consequent increase of contact pressure between the surfaces in contact. It leads to a generalized increase of the friction curve, with a more significant increase at very low speeds, i.e. in the BL regime.

Lubricant. In lubricated mechanisms, both the thickness of the lubricant layer and its viscosity play an important role in the resulting friction properties. The higher viscosity leads to higher shear forces and therefore higher friction levels in the EHL regime.

Temperature. The viscosity is also dependent on the temperature of the lubricant (Seeton, 2006), the higher the temperature, the lower the viscosity. This can be observed in Figure 3.2b with a decrease of friction in the EHL regime at higher temperatures. The effects of temperature are however more complex, changing also the BL and ML regimes. A possible explanation is that temperature also considerably affects the interaction forces of the surfaces in contact. This could be caused, e.g., by an asymmetric dilation of the gearbox components. 


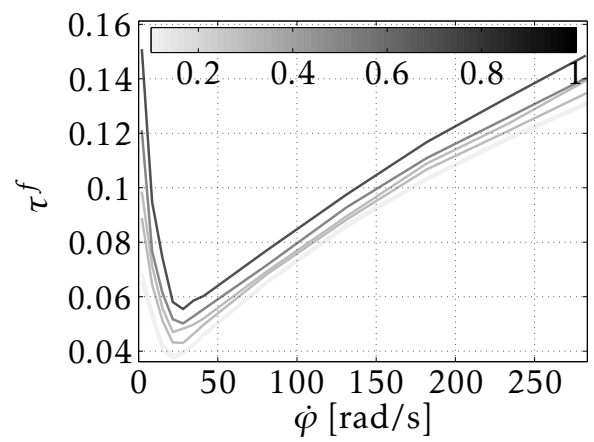

(a) Normalized load torques. A generalized bias-like increase with pronounced increases in the $B L$ regime.

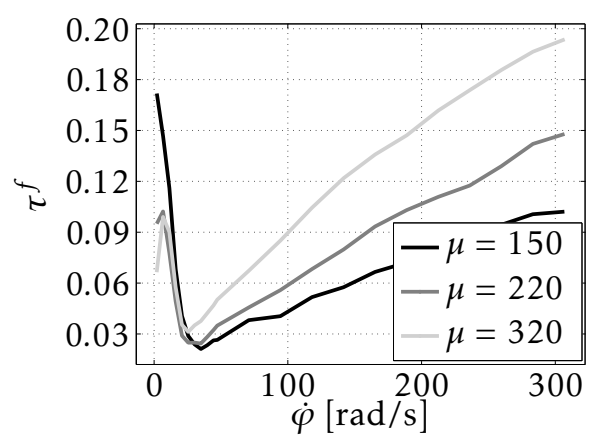

(c) Gearbox lubricant. Increases in the EHL region with kinematic viscosity. The viscosity values are in $\mathrm{mm}^{2} / \mathrm{s}$ and at 40 $C^{\circ}$.

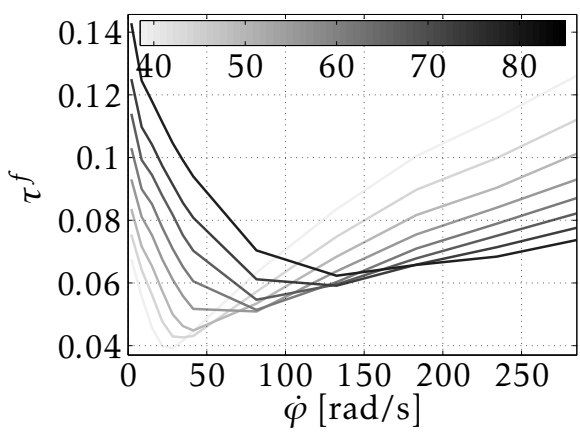

(b) Temperature $\left(\mathrm{C}^{\circ}\right)$. Significant increases in the $B L$ and $M L$ regions and decreases in the EHL region.

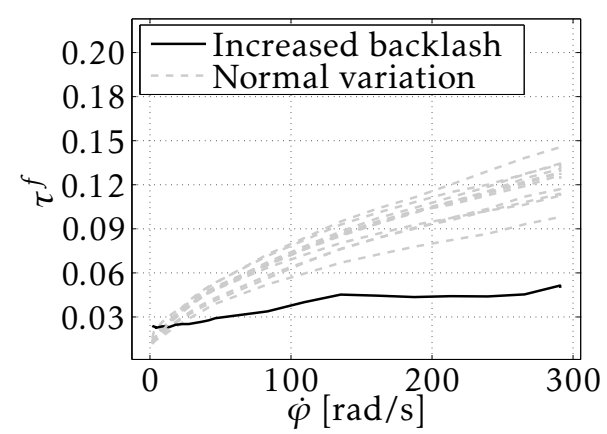

(d) Backlash for different robot individuals. Decreases in the ML and EHL regions.

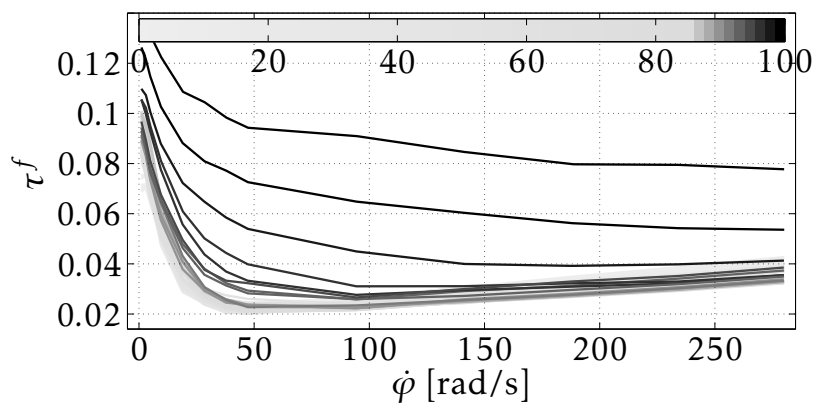

(e) Wear. Increases concentrated in the $M L$ region followed by a generalized increase. The colormap relates to the length of accelerated wear tests.

Figure 3.2: Effects of different factors to the friction curve. 
Wear. The increase of friction with wear as seen in Figure 3.2e is related to, amongst others, the accumulation of wear debris in the circulant lubricant. At early stages, the changes are observed specially in the ML regime, followed by generalized increases.

Backlash. The decrease of friction with backlash seen in Figure 3.2d can possibly be explained by a consequent loosening of the gearbox components, yielding lower contact pressures. Notice that backlash might follow from a degenerate wear process, where the amount of material removed by wear starts to be significant enough to create undesired clearances between the surfaces.

\subsection{Modeling}

Due to the complex nature of friction in a robot joint, it is common to accept models based on empirical observations of the phenomena. The history of the development of empirical friction models is extensive, see e.g. Dowson (1998). At a contact level, the surfaces' asperities can be compared to bristles on a brush. Each of these (stiff) bristles can be seen as a body with its own dynamics which are connected by a similar bulk. Different models have been proposed to model this dynamic behavior of friction, and some examples are presented in Harnoy et al. (2008); Al-Bender and Swevers (2008); Åström and Canudas-de Wit (2008). A typical approach is to consider all the dynamics into a single state (Dupont et al., 2002).

The LuGre fricion model, Olsson et al. (1998), is a common choice of dynamic models in robotics. For a revolute joint, the friction torque is given by the LuGre model as

$$
\begin{aligned}
\tau^{f} & =\sigma_{0} z+\sigma_{1} \dot{z}+h(\dot{\varphi}) \\
\dot{z} & =\dot{\varphi}-\sigma_{0} \frac{|\dot{\varphi}|}{g(\dot{\varphi})} z,
\end{aligned}
$$

where the state $z$ captures the average dynamic behavior of the asperities. It can be interpreted as their average deflection, with stiffness $\sigma_{0}$ and damping $\sigma_{1}$.

Since $z$ is not measurable, it is difficult to estimate the parameters describing the dynamic behavior of friction, i.e. $\left[\sigma_{0}, \sigma_{1}\right]$. In practice, it is common to accept only a static description of (3.1). In steady-state, (3.1) is equivalent to the static model:

$$
\tau^{f}(\dot{\varphi})=g(\dot{\varphi}) \operatorname{sign}(\dot{\varphi})+h(\dot{\varphi})
$$

which is fully described by the $g$ - and $h$ functions. In fact, (3.1) simply adds dynamics to (3.2).

The function $h(\dot{\varphi})$ represents friction in the EHL regime, where friction has a velocity strengthening behavior. For Newtonian fluids this behavior is directly 


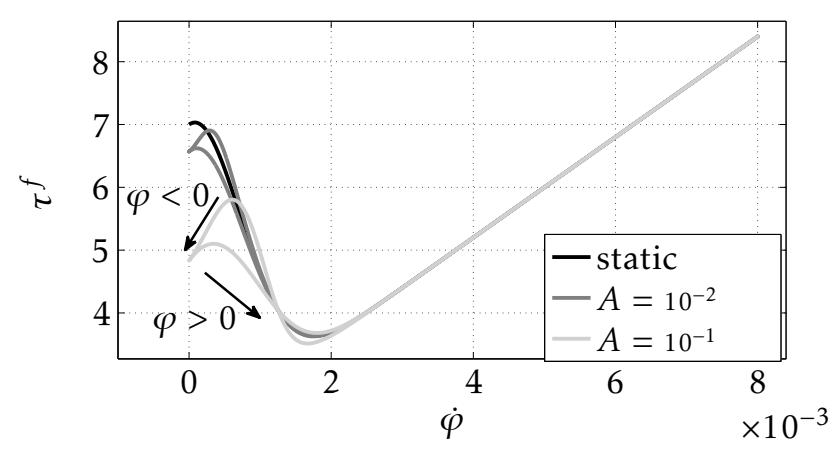

Figure 3.3: Simulation of a LuGre model under different acceleration levels $A$ and the related static friction model. The parameters are chosen for illustrative purposes with static parameters $\left[f_{c}, f_{s}, f_{v}, \dot{\varphi}_{s}, \alpha\right]=\left[2,5,810^{2}, 110^{-3}, 2\right]$ and dynamic parameters $\left[\sigma_{0}, \sigma_{1}\right]=\left[1.410^{6}, 2.4210^{3}\right]$.

proportional to speed, yielding the relationship

$$
h(\dot{\varphi})=f_{v} \dot{\varphi}
$$

for the viscous behavior of friction. The function $g(\dot{\varphi})$ captures the BL and ML regimes, where friction has a velocity weakening behavior. Motivated by the observations mainly attributed to Stribeck (Jacobson, 2003; Woydt and Wäsche, 2010; Bo and Pavelescu, 1982), $g(\dot{\varphi})$ is usually modeled as

$$
g(\dot{\varphi})=f_{c}+f_{s} e^{-\left|\frac{\dot{\varphi}}{\varphi_{s}}\right|^{\alpha}},
$$

where $f_{c}$ is the Coulomb friction, $f_{s}$ is defined as the standstill friction parameter ${ }^{\mathrm{i}}$, $\dot{\varphi}_{s}$ is the Stribeck velocity, and $\alpha$ is the exponent of the Stribeck nonlinearity. The resulting static friction model is given by

$$
\tau_{f}(\dot{\varphi})=\left[f_{c}+f_{s} e^{-\left|\frac{\dot{\varphi}}{\dot{\varphi}_{s}}\right|^{\alpha}}\right] \operatorname{sign}(\dot{\varphi})+f_{v} \dot{\varphi} .
$$

which can describe many of the friction characteristics with speed. This model structure is commonly used and was described in Bo and Pavelescu (1982). For the fixed $\alpha=1$, the model simplifies to the Tustin model, introduced by Tustin (1947). Notice that different choices of the $g$ - and $h$ are possible in the LuGre model.

Figure 3.3 shows the response of the LuGre model and the corresponding static model with $g-$ and $h$ chosen according to (3.4) and (3.3). The simulation was performed with $\dot{\varphi}$ as half a period of a triangular waveform with different slopes $A$. In the acceleration phase, the transition from BL to EHL gives less friction torques than during deceleration. The higher $A$, the more pronounced are the dynamic effects.

\footnotetext{
${ }^{\mathrm{i}} f_{S}$ is commonly called static friction parameter. An alternative nomenclature was adopted to make a distinction between the dynamic/static friction description.
} 


\subsection{Summary and Connections}

This chapter presented a brief overview of friction and wear from both empirical and phenomenological perspectives. The summary of the effects of different factors to the friction curves in Figure 3.2 gives a good idea behind the motivation and challenges of this work. The effects of temperature, load and wear in the figures are in comparable orders of magnitude. Attempting to determine the wear status based on observed changes to friction is therefore challenging. Load and temperature changes will always be present in applications and the diagnosis solutions must be able to cope with them.

The models presented in this chapter are only dependent on speed (and $z$ ). Extended joint friction models are proposed in Papers A and B to describe the effects of load, temperature and wear to friction. More realistic friction models are important for control, simulation and diagnosis of industrial robots. These models are used during the design and verification of the wear diagnosis methods proposed in Papers B and C. Simulation studies are a cost and time efficient alternative to wear experiments and have allowed for a more detailed analysis and evaluation of the diagnosis solutions in Paper D. 



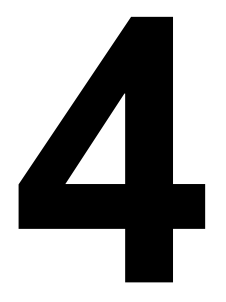

\section{Basics of Fault Diagnosis}

Fault diagnosis concerns the detection of an abnormal behavior and determination of its cause based on domain knowledge, premises and observations. Fault diagnosis is a multidisciplinary topic and of relevance in many different fields. The related literature is vast and the terminology and approaches can considerably vary across the different communities. With origins closer to control theory and statistical decision making, the field is known as Fault Detection and Isolation (FDI) (Isermann, 2006; Ding, 2013). In artificial intelligence, diagnosis is studied by the DX (Diagnosis) community (Reiter, 1987; De Kleer and Williams, 1987; Cordier et al., 2004). The area recognized as condition monitoring provides important tools for diagnosis and has origins in Maintenance Engineering (Rao, 1998). In Machine Learning, fault diagnosis can relate to classification problems (Kotsiantis, 2007).

It is outside the scope of this thesis to provide an extensive discussion of the different approaches and terminology used in fault diagnosis. Instead, the presentation aims at familiarizing the reader with the problems and contextualizing the methods developed in this work. The presentation and terminology used are closest to the ones found in the Fault Detection and Isolation literature and is mainly based in Gustafsson (2000); Isermann (2006); Basseville and Nikiforov (1993); Ljung (1999).

\subsection{Overview of Fault Diagnosis}

The fault diagnosis process can be divided in two main functions as depicted in Figure 4.1. In fault detection, data collected from the monitored system are processed and compared to available knowledge about the system to determine 


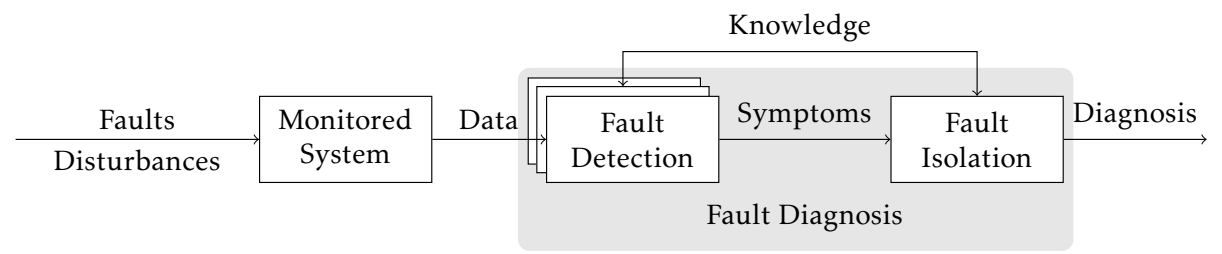

Figure 4.1: Overview of the fault diagnosis process. Data collected from the system are processed by the fault detection methods to generate symptoms that indicate the presence of abnormalities. The symptoms are analyzed in fault isolation to produce a diagnosis consistent to the knowledge embedded in the diagnosis solution and observations.

presence of abnormalities. Symptoms are the outputs of fault detection and are manifestations of one or more faults, indicating presence of abnormalities. Diagnosis of complex systems typically makes use of several fault detection methods, each based on partial information of the system. Each possible fault manifests itself in subsets of all the possible symptoms. In fault isolation, this information is used to find the faults that are consistent to all observed symptoms, i.e., it generates a diagnosis. Some fault diagnosis schemes also include fault identification where the size and the time profile of the faults are determined.

This work is mainly concerned with the design and evaluation of fault detection methods. This is motivated from the robotics application where the objective is to generate symptoms that can relate to an abnormal friction behavior caused by excessive wear levels. A determination of which component in the joint that is faulty, e.g., whether a bearing or a shaft, is of less importance. It is important though that a faulty state is detected in an early stage, so that appropriate maintenance actions can be scheduled before a failure. The discussion for the remaining part of this chapter is focused on the design of fault detection methods. For further information on fault isolation, the reader is referred to Isermann (2006); De Kleer and Williams (1987).

\subsubsection{Fault detection}

An overview of fault detection can be seen in Figure 4.2. The monitored system is affected by faults and disturbances and generates data. The data are processed to extract characteristic features (properties) of the system (e.g., parameters, residuals, signal spectra). The behavior of the extracted features is compared to their behavior found under nominal conditions, generating one or more test quantities. The test quantities measure how far the observed features are from the nominal case. Test quantities are input to a decision rule (e.g., a threshold check or a statistical test) to determine presence of an abnormality, i.e., it generates symptoms.

The combined tasks of feature extraction and behavior comparison are performed by the fault detection algorithm (FDA). Depending on the strategy to fault detec- 


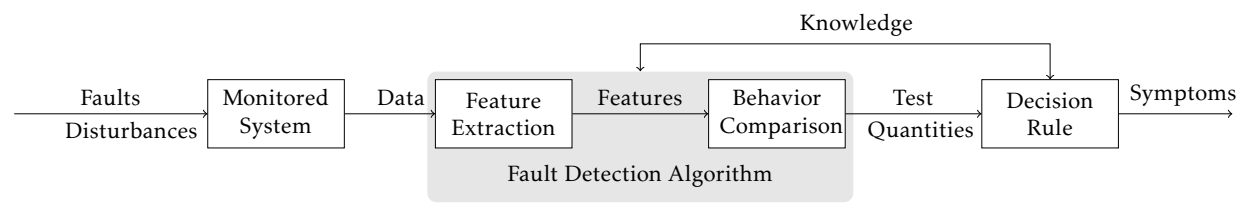

Figure 4.2: Overview of a fault detection scheme. The monitored system is affected by inputs, e.g., faults and disturbances, and generates data. Features are extracted from the data which are compared against their nominal behaviors, generating test quantities. The test quantity measures conformity between the observed and nominal behavior of the features which is tested for by the decision rule, generating a symptom.

tion, there is little distinction between the different tasks in fault detection, e.g., a classifier makes a direct map from data to a symptom or to a diagnosis and thus involves feature extraction, behavior comparison and a decision rule. The division in subtasks is made to fit the proposed methods into a common framework.

When fault detection is performed with no perturbation of the system's functions, during operation, it is called an on-line solution, otherwise it is denoted off-line, as "off-the-line", and will reduce the system's availability. When fault detection is performed by actively exciting the system, it is called active. If it is performed by passively studying the system, it is called passive. When fault detection is performed at each new observation (e.g., at each incoming data samples) it is a sequential solution, otherwise it is a batch solution. Sequential solutions with finite memory and computational requirements are also denoted as recursive and are important for implementation issues.

\section{Example 4.1: An off-line passive method for wear monitoring}

The wear processes inside a robot joint cause an eventual increase of wear debris in the lubricant. The iron content of lubricant samples taken from the robot joint can thus be used to indicate the condition of the joint. The study of wear debris is known as ferrography and was first introduced by Seifert and Westcott (1972). Since then, the science has evolved and helped to understand wear related phenomena, see Roylance (2005) for a historical review. In Figure 4.3, different types of wear particles are shown. In most applications, the collection of lubricant samples can only be performed when the system is turned off, in an off-line manner, followed by laboratory analysis. Notice that no dedicated excitation of the system is needed, so this is also a passive method.

The design of fault detection methods makes extensive use of knowledge about the monitored system. This knowledge might come from models, assumptions, data, an operator, an expert, etc. The next section presents an important type of knowledge representation, models of systems and faults. In general, the extent to which a system model is known can considerably affect the design and 


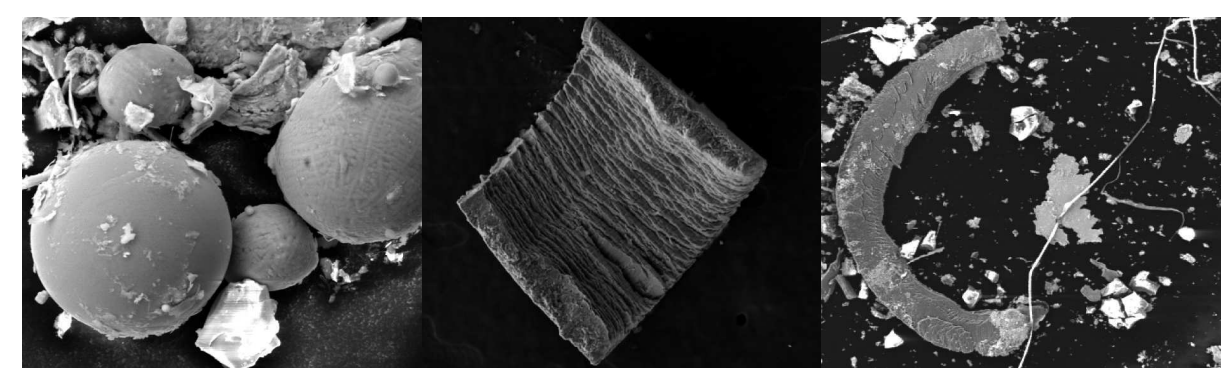

(a) Spherical.

(b) Laminar.

(c) Cutting.

Figure 4.3: Images of different types of wear particles from ferrography. The mechanical condition of the system may be determined from analysis of the characteristics of the wear particles, e.g., the type, shape, frequency, etc. (Pictures extracted from Machalíková et al. (2010)).

performance of diagnosis solutions.

\subsubsection{Models of systems and faults}

In order to choose the diagnosis solution, it is important to understand the behavior of the system and its dependencies on the faults. This can be achieved with the use of models. A system model describes the relationships between the variables affecting the system. The measured output data is denoted by $y$ which is affected by deterministic, $z$, and random, $v$, inputs. The relationship between the variables is described by a map

$$
y=\boldsymbol{h}(z, v) \text {. }
$$

The random inputs $v$ are unknown (e.g., noise), while $z$ could have both known and unknown components. The known components of $z$ include control inputs $\boldsymbol{u}$ and reference signals $\boldsymbol{r}$. Unknown components of $\boldsymbol{z}$ include disturbances $\boldsymbol{d}$ and faults $f$. The known signals $y, \boldsymbol{u}$ and $\boldsymbol{r}$ are the data input to the diagnosis process. For the design and evaluation of diagnosis methods, it is important to understand how the unknown components $\boldsymbol{v}, \boldsymbol{d}$ and $\boldsymbol{f}$ affect the available data so that the different effects can be identified correctly.

When the system model map is a function of some parameters $\theta$, this map is called a model structure, $\mathcal{M}$,

$$
\mathcal{M}: \quad y=\boldsymbol{h}(z, v ; \theta) .
$$

A particular choice of parameters, $\theta^{\prime}$, leads to a model instance, $\mathcal{M}\left(\boldsymbol{\theta}^{\prime}\right)$, of the model structure $\mathcal{M}$. Modeling can be performed based on first principles, e.g., from the laws of physics, where the parameters of the resulting model will have some physical meaning. In case there are parameters with unknown values, these can be determined empirically, e.g., from an identification procedure. An alternative to modeling from first principles is to choose the model based on how well it 
describes the data, where the parameters have no obvious physical interpretation.

Fault Models. Of special importance is the modeling of faults. The fault model chosen must reflect the physical effects of the fault. Faults can be categorized by their time behavior and by the manner they affect the system (Isermann, 2006). With respect to the time behavior, fault models are often categorized as:

Abrupt, affect the system abruptly, stepwise.

Incipient, develop gradually with time.

Intermittent, affect the system with interruptions.

According to how they affect the system, fault models are categorized as:

Additive, effectively added to the signals describing the model.

Multiplicative, acting on a parameter of the system. For example, changing a parameter $\theta$ of the model structure $\mathcal{M}$.

Structural, introduces new governing terms to the describing equations of the system. For example, changing the model structure $\mathcal{M}$.

The choice of fault model can be motivated from the physical process in which faults take part. For example, a bias like error in a sensor can be modeled as an additive fault, while an incorrect specification of a payload mass for a robot can by modeled as a multiplicative fault since it affects a parameter for the model describing its dynamics.

\section{Example 4.2: An industrial robot under wear and temperature effects}

With references to Section 2.2 and Chapter 3, a manipulator can be described in a simplified manner by a multi-body rigid model

$$
M(\varphi) \ddot{\varphi}+C(\varphi, \dot{\varphi}) \dot{\varphi}+\tau^{g}(\varphi)+\tau^{f}\left(\dot{\varphi}, \tau^{\ell}, \xi, \omega\right)=\tau
$$

where the parametric dependencies are not shown for simplicity. The friction torques $\tau^{f}(\cdot)$ are described as a function of angular speed $\dot{\varphi}$, manipulation load torque, $\tau^{\ell}$, temperature, $\xi$, and wear, $\omega$. The fault $f$ relates to the wear levels $\omega$. Wear changes the behavior of friction in a gradual manner and can be modeled as a change in the friction model parameters, in which case it is an incipient multiplicative fault. The measured (known) outputs $y$ are the angular positions $\varphi$, from which is also possible to achieve angular speeds $\dot{\varphi}$. The control inputs $\boldsymbol{u}$ are the applied torques ${ }^{\mathrm{i}} \boldsymbol{\tau}$ which depend on a reference signal, $\boldsymbol{r}$, not described in (4.3). The measured quantities are corrupted by noise, $v$, not described in (4.3). The loads, $\tau^{\ell}$, and temperatures, $\xi$, are unknown and considered as disturbances $d$.

\footnotetext{
${ }^{\mathrm{i}}$ Based on the simplification that the relation between current and applied torque is given by a constant. See Section 2.4 for details.
} 


\subsection{Fault Detection Algorithms}

An important tool to support the design of fault detection algorithms is a system model structure $\mathcal{M}$. Two approaches based on a system model are briefly discussed.

Residuals. A nominal model instance, $\mathcal{M}\left(\theta^{0}\right)$, is used to reconstruct the output from the data, creating an analytical redundancy $\widehat{y}\left(k, \theta^{0}\right)$ of the system outputs $y_{k}$ at each sample time $k$. The difference

$$
\varepsilon\left(k, \theta^{0}\right)=y_{k}-\widehat{y}\left(k, \theta^{0}\right),
$$

also known as the model residuals measures deviations between the model and observations and can be used directly as test quantities. The modelbased design of residual generation methods, also known as fault detection filters, has received much attention in the literature, see e.g., Frank and Ding (1997); Liu and Zhou (2008); Ding (2013).

Parameter estimation. The unknown parameters for the model instance, $\mathcal{M}(\boldsymbol{\theta})$, are chosen such that the resulting model best explains the data in some way. For instance, the least squares criterion chooses $\theta$ such that the sum of squared errors is minimized, i.e.,

$$
\widehat{\boldsymbol{\theta}}=\arg \min _{\boldsymbol{\theta}} \sum_{k}\left(y_{k}-\widehat{y}(k, \theta)\right)^{2} .
$$

Test quantities can be defined based on a comparison between $\widehat{\boldsymbol{\theta}}$ and a nominal region for the parameters $\Theta^{0}$.

Given an estimate $\widehat{\boldsymbol{\theta}}$, the residuals $\varepsilon(\widehat{\boldsymbol{\theta}})=y-\widehat{y}(\widehat{\boldsymbol{\theta}})$ can also be used as test quantities which are suitable for diagnosis of structural faults.

Remark 4.1. Parameter estimation techniques are a natural choice for multiplicative and structural fault models, while the typical formulation for residual generation considers additive fault models. Nevertheless, these methods can be used interchangeably, Isermann (2006).

When a system model structure is not available, alternative solutions are possible. These solutions will typically require expert knowledge about the data or extra (redundant) sensor information. An example of such expert knowledge is found in the analysis of features of measured signals, e.g., their frequency responses. Some fault detection algorithms that relate to the research results presented in this thesis are described in more details next.

\subsubsection{Parameter estimation}

The objective is to identify the unknown parameters of a known model structure from the data. Algorithmically, the solutions will depend on the model structure and whether a recursive method is sought. To illustrate this class of methods, the maximum likelihood approach is described next. The presentation is based on 
Ljung (1999).

Let the mechanism generating the data $y_{k}$ be described by its probability density $p\left(y_{k} \mid \theta\right)$ which is a function of unknown parameters $\theta$. For a sequence of observations $Y_{N}=\left[\begin{array}{lllll}y_{1}, & \cdots, & y_{k}, & \cdots & y_{N}\end{array}\right]$, the joint density is denoted $p_{N}\left(Y_{N} \mid \theta\right)$. Given a particular observation of the data $Y_{N}^{*}$, the likelihood function is defined as the joint data density evaluated at $Y_{N}^{*}$ as a function of the parameters $\theta$, i.e., $\mathcal{L}(\boldsymbol{\theta}) \triangleq p_{N}\left(Y_{N}^{*} \mid \boldsymbol{\theta}\right)$. The likelihood function $\mathcal{L}(\boldsymbol{\theta})$ relates to the probability of an observation $Y_{N}^{*}$ for a certain choice of parameter $\theta$. The maximum likelihood estimate is defined as the parameter value maximizing the likelihood function

$$
\widehat{\theta}=\arg \max _{\theta} \mathcal{L}(\theta)=\arg \max _{\theta} \log \mathcal{L}(\theta)
$$

where the last equivalence follows since maximizing a function is equivalent to maximizing its logarithm (a monotonic transformation).

To justify the use of the maximum likelihood estimate, consider first the following criterion for assessing the performance of an estimator. Let the true parameter be denoted by $\theta^{0}$, the quality of an estimate $\widehat{\theta}$ can be assessed by the mean square error matrix, defined as

$$
P=\mathbb{E}\left[\left(\widehat{\boldsymbol{\theta}}-\boldsymbol{\theta}^{0}\right)\left(\widehat{\boldsymbol{\theta}}-\boldsymbol{\theta}^{0}\right)^{T}\right]=\Sigma_{\widehat{\boldsymbol{\theta}}}+\boldsymbol{b}(\widehat{\boldsymbol{\theta}}) \boldsymbol{b}(\widehat{\boldsymbol{\theta}})^{T},
$$

where $\Sigma_{\widehat{\boldsymbol{\theta}}}$ is the covariance matrix and $\boldsymbol{b}(\widehat{\boldsymbol{\theta}})$ is the bias defined respectively as

$$
\Sigma_{\widehat{\boldsymbol{\theta}}} \triangleq E\left[(\widehat{\boldsymbol{\theta}}-\mathbb{E}[\widehat{\boldsymbol{\theta}}])(\widehat{\boldsymbol{\theta}}-\mathbb{E}[\widehat{\boldsymbol{\theta}}])^{T}\right], \quad \boldsymbol{b}(\widehat{\boldsymbol{\theta}}) \triangleq \mathbb{E}[\widehat{\boldsymbol{\theta}}]-\boldsymbol{\theta}^{0}
$$

For any unbiased estimator, i.e., $\boldsymbol{b}(\widehat{\boldsymbol{\theta}})=\mathbf{0}$, the following inequality, known as the Cramér-Rao lower bound, applies,

$$
P \geq \mathcal{F}\left(\boldsymbol{\theta}^{0}\right)^{-1}, \quad \mathcal{F}\left(\boldsymbol{\theta}^{0}\right) \triangleq \mathbb{E}\left[\left.\left(\frac{\partial \log \mathcal{L}(\boldsymbol{\theta})}{\partial \boldsymbol{\theta}}\right)\left(\frac{\partial \log \mathcal{L}(\boldsymbol{\theta})}{\partial \boldsymbol{\theta}}\right)^{T}\right|_{\boldsymbol{\theta}=\boldsymbol{\theta}^{0}}\right],
$$

where $\mathcal{F}\left(\theta^{0}\right)$ is known as the Fisher information matrix. Note here that a sensible approach to experiment design is, if possible, to affect the likelihood function such that the inverse Fisher information matrix is made small in some sense, thus improving the achievable performance for any unbiased estimator.

Suppose now that each data $y_{k}$ are independently and identically distributed so that the joint density $p_{N}\left(Y_{N} \mid \boldsymbol{\theta}\right)=\prod_{k=1}^{N} p\left(y_{k} \mid \boldsymbol{\theta}\right)$. Then, as the number of data tends to infinity, $N \rightarrow \infty$, the maximum likelihood estimate given by (4.4) converges in distribution to a Gaussian centered around $\theta^{0}$ with covariance given by the inverse of the Fisher information matrix,

$$
\sqrt{N}\left(\widehat{\boldsymbol{\theta}}-\boldsymbol{\theta}^{0}\right) \sim A s \mathcal{N}\left(\mathbf{0}, \mathcal{F}\left(\boldsymbol{\theta}^{0}\right)^{-1}\right) .
$$

That is, the maximum likelihood estimate is asymptotically unbiased and has the smallest possible covariance, achieving the Cramér-Rao lower bound. The 
maximum likelihood estimate is illustrated in Example 4.3.

Example 4.3: Scalar linear regression under additive Gaussian noise

Let the model for each datum $y_{k}$ be described by a linear regression with additive uncertainty $v_{k}$,

$$
y_{k}=\phi(k)^{T} \theta^{0}+v_{k}
$$

where $\phi(k): \mathbb{R}^{d} \mapsto \mathbb{R}$ is a deterministic function and $\theta^{0} \in \mathbb{R}^{d}$ are the true parameters. For $N$ observations, $y \in \mathbb{R}^{N}$, the model can be written in vector form as

$$
y=\Phi \theta^{0}+v, \quad \Phi \triangleq[\phi(1), \quad \ldots, \quad \phi(N)]^{T} .
$$

Considering $v$ as a zero mean Gaussian uncertainty with covariance $Q^{-1}$, i.e., $v \sim \mathcal{N}\left(\mathbf{0}, Q^{-1}\right)$, the joint density is then $p_{N}\left(y \mid \theta^{0}\right)=\mathcal{N}\left(y ; \Phi \theta^{0}, Q^{-1}\right)$, where the Gaussian distribution is defined as

$$
\mathcal{N}(\boldsymbol{x} ; \boldsymbol{\mu}, \Sigma) \triangleq|2 \pi \Sigma|^{-1 / 2} e^{-\frac{1}{2}(\boldsymbol{x}-\boldsymbol{\mu})^{T} \Sigma^{-1}(\boldsymbol{x}-\boldsymbol{\mu})} .
$$

For a given observation $y^{*}$, the maximum likelihood estimate of the parameters reduces to a weighted linear least squares and is given by

$$
\begin{aligned}
\widehat{\boldsymbol{\theta}} & =\arg \max _{\boldsymbol{\theta}} \log \mathcal{N}\left(y^{*} ; \Phi \theta, Q^{-1}\right) \\
& =\arg \min _{\boldsymbol{\theta}} \frac{1}{2}\left(y^{*}-\Phi \theta\right)^{T} Q\left(y^{*}-\Phi \boldsymbol{\theta}\right)=\left(\Phi^{T} Q \Phi\right)^{-1} \Phi^{T} Q y^{*} .
\end{aligned}
$$

Because $\widehat{\theta}$ is a linear function of the data $y^{*}$, it is also a Gaussian distributed random variable and is given by

$$
\widehat{\boldsymbol{\theta}} \sim \mathcal{N}\left(\boldsymbol{\theta}^{0}, \Sigma_{\widehat{\boldsymbol{\theta}}}\right), \quad \Sigma_{\widehat{\boldsymbol{\theta}}} \triangleq\left(\Phi^{T} Q \Phi\right)^{-1},
$$

the estimate is thus unbiased and it is possible to show that $\Sigma_{\widehat{\theta}}$ achieves the Cramér-Rao lower bound even for finite $N$.

Behavior comparison. The result in (4.8) suggests the generation of test quantities in two different manners. Either the $d$ estimated parameters $\widehat{\theta} \in \mathbb{R}^{d}$ are compared to nominal values $\theta^{0}$ one by one, or they are compared jointly. In the first case, the following test quantity can be used for each of the $i$ th parameter

$$
q=\frac{[\widehat{\boldsymbol{\theta}}]_{i}-\left[\boldsymbol{\theta}^{0}\right]_{i}}{\sqrt{\left[\Sigma_{\widehat{\boldsymbol{\theta}}}\right]_{i i}}} \sim A s \mathcal{N}(0,1), \quad 1<i<d .
$$

In the second case, the following test quantity can be used

$$
q=\left(\widehat{\boldsymbol{\theta}}-\boldsymbol{\theta}^{0}\right)^{T} \Sigma_{\widehat{\boldsymbol{\theta}}}^{-1}\left(\widehat{\boldsymbol{\theta}}-\boldsymbol{\theta}^{0}\right) \sim A s \mathcal{X}_{d}^{2},
$$

where $\mathcal{X}_{d}^{2}$ is the chi-square distribution with $d$ degrees of freedom. Because the (asymptotic) distribution for the test quantities are known under the nominal 


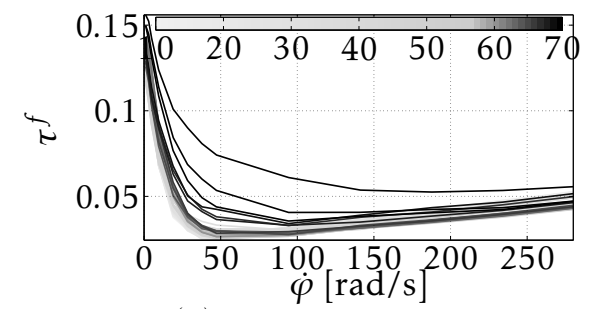

(a) Friction curves.

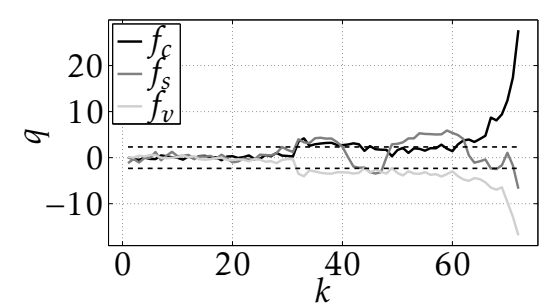

(b) Test quantities as in (4.14).

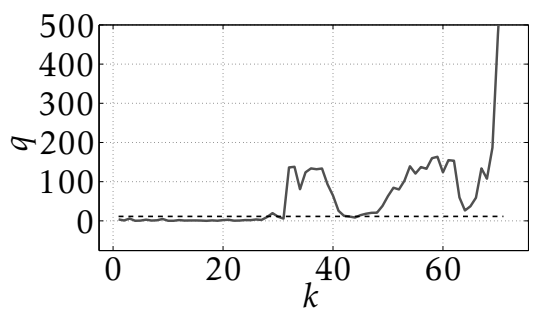

(c) Test quantity as in (4.15).

Figure 4.4: Test quantities for the detection of changes in the parameters of a friction model. The friction data are shown in (a) with colormap relating to the experimentation index $k$. For each friction curve, i.e., for each $k$, the parameters of a friction model are estimated and the test quantities defined in (4.14) and (4.15) are computed and shown in figures (b) and (c) respectively.

case, a decision rule is readily available from confidence intervals of the related distributions. In some cases, such as in Example 4.3, the confidence intervals are exact for finite $N$. A test based on (4.15) is used for example in Peretzki et al. (2011) to determine whether estimated parameters significantly deviate from zero, i.e., $\theta^{0}=\mathbf{0}$, and the test quantity is used as a measure of the data quality for identification. Example 4.4 illustrates the use of parameter estimates for detection of changes in the parameters of a friction model.

Example 4.4: Friction change detection for a robot joint under wear effects $\neg$ The Tustin friction model presented in Section 3.3 is a common choice to describe the behavior of friction. For a fixed Stribeck velocity, $\dot{\varphi}_{s}$, and for positive speed values, the Tustin model can be written as a linear regression

$$
\tau^{f}(\dot{\varphi}, \boldsymbol{\theta})=f_{c}+f_{s} e^{-\frac{\dot{\varphi}}{\dot{\varphi}_{s}}}+f_{v} \dot{\varphi}=\left[\begin{array}{lll}
1, & e^{-\frac{\dot{\varphi}}{\varphi_{s}}}, \quad \dot{\varphi}
\end{array}\right]^{T}\left[\begin{array}{lll}
f_{c}, & f_{s}, & f_{v}
\end{array}\right]=\phi(\dot{\varphi})^{T} \boldsymbol{\theta} .
$$

A dedicated experiment is used to collect friction data, $\tau^{f} \in \mathbb{R}^{N}$, from a robot joint. From this experiment $N=13$ friction data points are retrieved. It is assumed that these data can be described by (4.16) under an additive zero mean Gaussian uncertainty with covariance $Q^{-1}=\gamma I$ and where the Stribeck speed is fixed to $\dot{\varphi}_{s}=12.85$. The resulting data model is of the form given in Example 4.3.

The data collection experiment is repeated $K=72$ times during accelerated wear tests and the resulting friction curves are shown in Figure 4.4a. At time $k$, max- 
imum likelihood estimates of the parameters are found as given by (4.12b). A nominal parameter value $\theta^{0}$ is assigned from healthy data and the test quantities based on (4.14) and (4.15) are computed using an estimate of $\gamma$ found from the data. The resulting test quantities are shown in Figures $4.4 \mathrm{~b}$ and $4.4 \mathrm{c}$ together with $99 \%$ confidence intervals. For these data, it is known that no significant effects of wear are present before $k=60$. However, as can be seen, the test quantities leave the confidence intervals around $k=30$ and $k=50$. A possible explanation for this behavior is that another variable other than speed, such as temperature, is causing the variations to friction and a more reliable alternative is needed to avoid false alarms.

\subsubsection{Signal-driven methods}

In many applications, the available data are signals. It is possible to extract information about the system condition by only considering characteristics of these signals. In order to reveal relevant features of the signals for fault detection, transforms are widely used in signal-driven methods. A transform is used to "map" a signal from its original domain to an alternative domain. Features of the data in the alternative domain may reveal more information about the faults.

An integral transform is any transform $\mathbf{T}$ of the form

$$
\bar{y}(v)=\mathbf{T}\{y(t)\}=\int_{t_{0}}^{t_{1}} \kappa(t, v) y(t) \mathrm{d} t .
$$

where $y(t)$ is the original signal, $\bar{y}(v)=\mathbf{T}\{y(t)\}$ is the transformed signal, function of $v$, and $\kappa(t, v)$ is a kernel function. Several types of integral transforms and discrete transforms can be defined, e.g., Fourier transform, Wavelet transform, Karhunen-Loève transform, Radon transform, etc. Each transform will highlight different properties of the data in the transformed domain.

For example, the Fourier transform $\mathbf{F}\{y(t)\}$ is a transform with

$$
\kappa(t, v)=e^{-\mathrm{i} t v}, \quad t_{0}=-\infty, \quad t_{1}=\infty .
$$

When $t$ is time, $v$ is frequency. The transformed signal $\bar{y}(v)=\mathbf{F}\{y(t)\}$, is said to be the frequency representation of $y(t)$. The analysis of data in the frequencydomain has found particular success in the monitoring of rotating machines, Taylor (1994); de Silva (2007). Example 4.5 illustrates the use of frequency domain analysis for monitoring of backlash in gearboxes.

\footnotetext{
Example 4.5: Backlash monitoring in the frequency domain

This example is based on Sander-Tavallaey and Saarinen (2009) where backlash is studied in drives equipped with compact gearboxes. An increase of backlash will introduce additional resonance peaks to the frequency content of the drive response. Spectral analysis can therefore be used to indicate backlash changes.
}

A dedicated test-cycle, displayed in Figure 4.5a, is used to excite the drive unit. In 


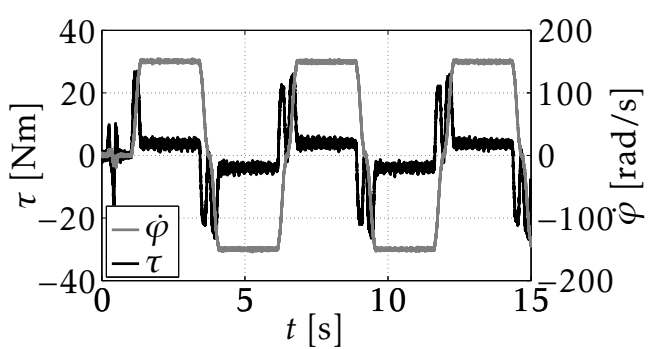

(a) Test-cycle excitation.

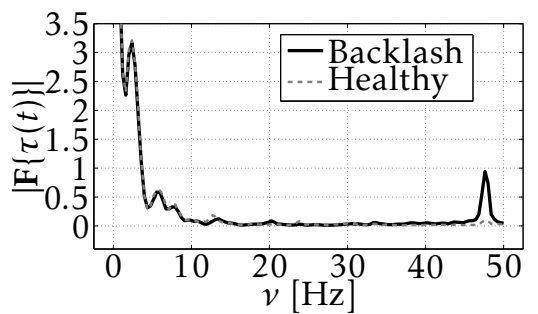

(b) Torque spectra.

Figure 4.5: Backlash monitoring through spectrum analysis. The drive is excited with a test-cycle in an off-line manner as displayed in (a). The frequency spectrum of the torque signals are shown in (b). Notice the increased resonance peak around $47 \mathrm{~Hz}$ for the unit with increased backlash compared to the healthy unit.

Figure 4.5b, spectra estimates for the torque signals are shown for a healthy unit and for a unit with increased backlash levels. As can be seen, there is an increase of the frequency response around $47 \mathrm{~Hz}$. In the paper, this deviation is used to generate a test quantity used for backlash monitoring. The proposed method takes only a few seconds to execute and does not consider additional vibration measurements, which are common for this type of method. Notice that this is an off-line active solution since it is based on a test-cycle.

Behavior comparison. As in Example 4.5, specific characteristics of the spectrum can be used as test quantities, allowing for an automated fault detection. With knowledge of the behavior of the entire spectrum under a certain condition, test quantities can also be defined by a direct comparison between spectra. For example, let $\bar{y}^{0}(v)$ be a spectrum representing a known behavior, e.g., fault-free, and $\bar{y}(v)$ be the spectrum found from incoming test data $y$, the log-spectral distance between them is

$$
q=\left\|\log \frac{\bar{y}^{0}(v)}{\bar{y}(v)}\right\|_{\delta},
$$

and can be used as a test quantity to determine conformity to the reference spectrum. The choice of norm will highlight different characteristics, e.g. $\delta=2$ leads to the mean quadratic distance and $\delta=\infty$ leads to the maximum deviation. See Basseville (1989) for more on spectral distances.

\subsubsection{Data-driven methods}

Statistical features extracted from the data are also valuable for fault diagnosis. Methods based on principal component analysis, partial least squares, linear discriminant analysis and classification methods have found success in many applications, see, e.g., Yin et al. (2012) for an overview of techniques. 
An important feature for data-driven methods is the distribution of the data $p(y)$, from which different test quantities can be defined. A density estimator relevant to this work is described next. The discussion follows with the presentation of methods defined in the distribution domain and a description of linear discriminant analysis.

\section{Kernel density estimator}

A nonparametric estimate of the distribution $p(y)$ based on the data vector $y$ can be achieved from the empirical characteristic function. For a scalar random variable with probability density function $p(y)$, the characteristic function $\vartheta(v): \mathbb{R} \rightarrow \mathbb{C}$ is defined as (Durrett, 2010):

$$
\vartheta(v)=\mathbb{E}\left[e^{\jmath v y}\right]=\int_{-\infty}^{\infty} e^{\jmath v y} p(y) \mathrm{d} y=\mathbf{F}^{-1}\{p(y)\} 2 \pi,
$$

where $\mathbf{F}^{-1}\{\cdot\}$ is the inverse Fourier transform. So the density function can be found from the characteristic function through its Fourier transform. Following Parzen (1962), given the sample $y \in \mathbb{R}^{N}$, the empirical estimate of $\vartheta(v)$ is

$$
\widehat{\vartheta}(v)=\frac{1}{N} \sum_{n=1}^{N} e^{\jmath v y_{n}},
$$

and the objective is to retrieve the density function from $\widehat{\vartheta}(v)$. This is essentially a spectrum estimation problem. A direct estimation of the density function from the Fourier transform of $\widehat{\vartheta}(v)$ will however lead to an estimate with variance that does not decrease with $N$ (Ljung, 1999, Section 6.4). To avoid this, $\widehat{\vartheta}(v)$ is multiplied with a weighting function $\psi_{h}(v)=\psi(h v)$. The weighting function is typically symmetric, satisfying $\psi(0)=1$ and tends to zero when $v$ tends to infinity. The density estimate is then given by

$$
\begin{aligned}
\widehat{p}(y) & =\frac{1}{2 \pi} \mathbf{F}\{\widehat{\vartheta}(v) \psi(h v)\}=\frac{1}{2 \pi} \int_{-\infty}^{\infty} e^{-\jmath v y} \widehat{\vartheta}(v) \psi(h v) \mathrm{d} v \\
& =\frac{1}{2 \pi} \int_{-\infty}^{\infty} \frac{1}{N} \sum_{n=1}^{N} e^{\jmath v\left(y_{n}-y\right)} \psi(h v) \mathrm{d} v=\frac{1}{N h} \sum_{n=1}^{N} \frac{1}{2 \pi} \int_{-\infty}^{\infty} e^{\jmath v\left(\frac{y_{n}-y}{h}\right)} \psi(h v) \mathrm{d}(h v) \\
& =\frac{1}{N h} \sum_{n=1}^{N} \kappa\left(\frac{y_{n}-y}{h}\right)=\frac{1}{N} \sum_{n=1}^{N} \kappa_{h}\left(y-y_{n}\right),
\end{aligned}
$$

where $\kappa_{h}(y) h=\mathbf{F}^{-1}\left\{\psi_{h}(v)\right\}$. The resulting estimate is known as a kernel density estimate (KDE) and can also be generalized to the multidimensional case (Cacoullos, 1966). The function $\kappa_{h}(y)$ is a kernel function, satisfying $\kappa_{h}(\cdot) \geq 0$ and that integrates to 1. Typical kernel functions and their Fourier transforms are shown in Figure 4.6. The bandwidth parameter $h$ controls the smoothness of the resulting estimate, increasing the smoothness for larger values of $h$. When $h \rightarrow 0$, the ker- 


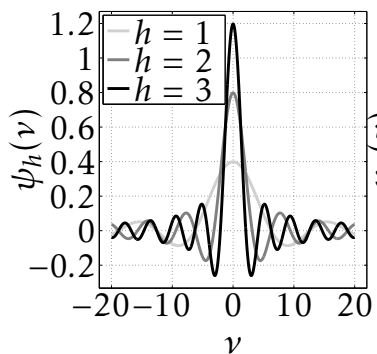

(a) Uniform.

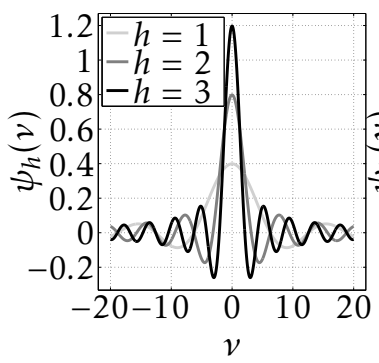

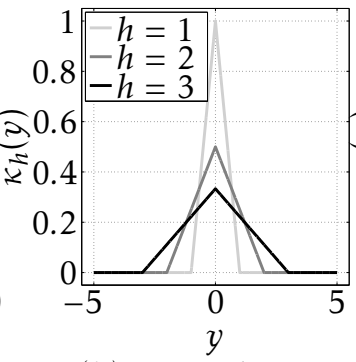

(b) Triangular.

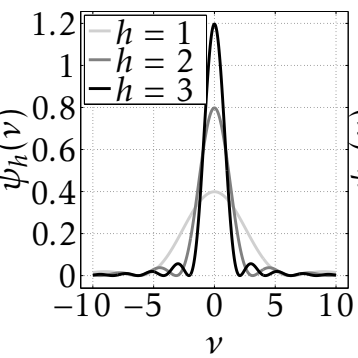

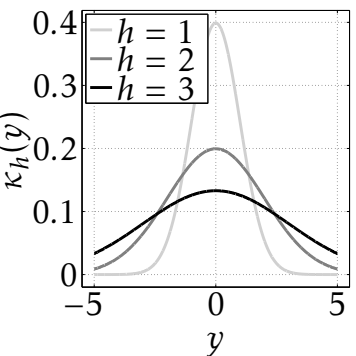

(c) Gaussian.

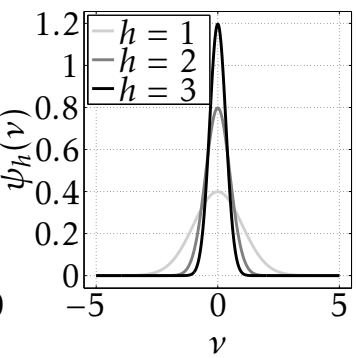

Figure 4.6: Kernel functions (upper row) and their respective Fourier transforms (bottom).

nel function approaches a Dirac delta and the resulting estimate will be a set of impulses located at the data points. For a detailed treatment of kernel density estimators and criteria/methods for choosing $h$ see Parzen (1962); Cacoullos (1966); Bowman and Azzalini (1997); Jones and Henderson (2009).

\section{Distribution domain methods}

Test quantities can be generated based on distribution domain features. This is a valid approach since the effects of faults often appear as changes in the data amplitude. Similar to spectrum analysis, test quantities can be generated from specific characteristics of the data distribution or from the entire distribution. Some approaches are discussed next.

Distribution peak. Rzeszucinski et al. (2012) propose the use of the peak of the Gaussian density as a test quantity for diagnosis of gearboxes based on vibration data. The test quantity is given by $q=1-\max _{y} p(y)$, where $p(y)$ is the (univariate) Gaussian density function. The Gaussian density has mode (maximum) at the mean $\mu$ and the test quantity simplifies to

$$
q=1-\max _{y} p(y)=1-p(\mu)=1-\frac{1}{\sqrt{2 \pi} \sigma},
$$

and depends only on an estimate of the standard deviation, $\sigma$, which is a measure of the data spread. 


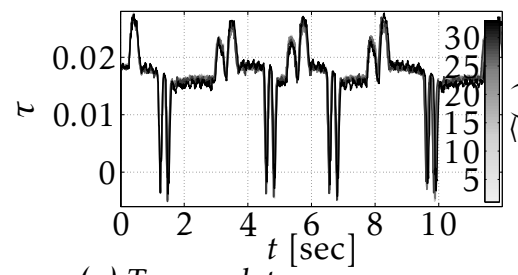

(a) Torque data sequences.

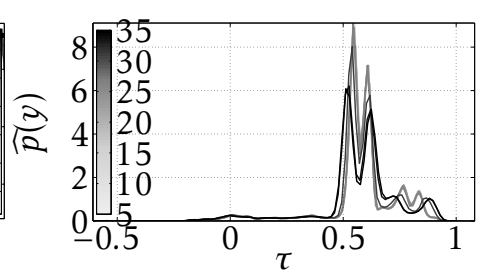

(b) Density estimates.

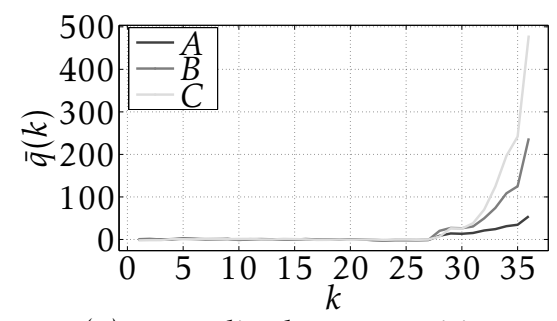

(c) Normalized test quantities.

Figure 4.7: Monitoring of a wear fault in an industrial robot joint in the distribution domain. The torque sequences are shown in (a) and their respective kernel density estimates are shown in (b); the colormaps relate to the experiment length $k$. The normalized response for the test quantities considered are shown in (c).

Data likelihood. Estimates of the data distribution can also be used to generate test quantities. With availability of a nominal data density estimate $\widehat{p}^{0}(y)$, test quantities can be generated based on the likelihood that incoming data $y$ present under this distribution. For batches of data $y \in \mathbb{R}^{N}$, a test quantity can be defined for instance as the (average negative log) likelihood under $\widehat{p}^{0}(y)$, i.e.

$$
q=-\frac{1}{N} \sum_{n}^{N} \log \widehat{p}^{0}\left(y_{n}\right) .
$$

Large values of this test quantity would indicate a change in behavior. This idea has been suggested by Agarwal (2007); Desforges et al. (2000); Yeung and Chow (2002) where flexible density models are used.

Comparison of distributions. An alternative is to compare a nominal density model $\widehat{p}^{0}(y)$ against a density estimate $\widehat{p}(y)$ achieved from test data. The comparison can be made with the use of distances between distributions, such as the symmetric Kullback-Leibler divergence, or Kullback-Leibler distance, given by

$$
q=\mathrm{KL}\left(\widehat{p}^{0}, \widehat{p}\right) \triangleq D_{\mathrm{KL}}\left(\widehat{p}^{0} \| \widehat{p}\right)+D_{\mathrm{KL}}\left(\widehat{p} \| \widehat{p}^{0}\right),
$$


where $D_{\mathrm{KL}}(\cdot \| \cdot)$ is the Kullback-Leibler divergence

$$
D_{\mathrm{KL}}\left(\widehat{p}^{0} \| \widehat{p}\right) \triangleq \int_{-\infty}^{\infty} \widehat{p}^{0}(y) \log \frac{\widehat{p}^{0}(y)}{\widehat{p}(y)} \mathrm{d} y .
$$

The test quantity in (4.23) is suggested in Paper $C$ for fault diagnosis. Other examples of divergences and distances are possible, see Reid and Williamson (2011); Basseville (1989) for more. Example (4.6) illustrates the use of different distribution domain test quantities for monitoring of wear in a robot joint.

Example 4.6: Distribution domain methods for wear monitoring in a robot Torque data collected from the execution of a test-cycle for a robot undergoing accelerated wear tests are considered. The data are shown in Figure 4.7a and contain $K=36$ batches of torque sequences, each containing $N=7440$ samples. For these data, it is known that no significant wear is present until batch $k=25$. Kernel density estimates are found for each data batch with a Gaussian kernel function and are displayed in Figure 4.7b. An inspection of the Figures 4.7a and $4.7 \mathrm{~b}$ reveals that the effects of the fault are more easily distinguishable in the distribution domain.

The test quantities described in Equations (4.21), (4.22) and (4.23) are computed for each batch $k$ and are denoted $A, B$ and $C$ respectively. For test quantities $B$ and $C$, the density estimates used are those shown in Figure 4.7b. The nominal density estimate $\widehat{p}^{0}(\tau)$ is assigned for the first batch $k=1$. For a comparison, each test quantity $q_{k}$ is normalized according to $\bar{q}_{k}=\left(q_{k}-\mu^{0}\right) / \sigma^{0}$ where $\mu^{0}$ and $\sigma^{0}$ are the mean and standard deviation of the test quantity computed for $k \leq 5$. The results are shown in Figure $4.7 c$, where can be seen that all test quantities respond to the wear changes while test quantity $C$ gives the clearest response.

\section{Linear discriminant analysis}

Given a data vector $y \in \mathbb{R}^{N}$, a discriminant function reduces the data dimension to $L<N$ according to a linear transformation

$$
\bar{y}=W^{T} y, \quad W=\left[\begin{array}{lll}
w_{1}, & \cdots, & w_{L}
\end{array}\right]
$$

where the weight matrix $W \in \mathbb{R}^{N \times L}$ is chosen based on a criterion to support the discrimination of different behaviors of the data, e.g., faulty or healthy. The resulting weighted data vector $\bar{y}$ can be used as test quantities for fault detection.

The weight matrix $W$ is chosen based on labeled data, i.e., data sets where the condition of the system is known. As an illustration, consider availability of data sets corresponding to the classes of normal and faulty operations

$$
Y_{K_{0}}^{0}=\left[\begin{array}{lll}
y_{1}^{0}, & \cdots, & y_{K_{0}}^{0}
\end{array}\right], \quad Y_{K_{f}}^{f}=\left[\begin{array}{lll}
y_{1}^{f}, & \cdots, & y_{K_{f}}^{f}
\end{array}\right]
$$

respectively. Take the case $L=1$ and $W=w$, the mean of $Y_{K_{0}}^{0}$ (and similarly for 
$\left.Y_{K_{f}}^{f}\right)$ in the transformed domain is

$$
\bar{\mu}^{0}=\frac{1}{K_{0}} \sum_{k}^{K_{0}} \bar{y}_{k}=\frac{1}{K_{0}} \sum_{k}^{K_{0}} w^{T} y_{k}^{0}=w^{T}\left(\frac{1}{K_{0}} \sum_{k}^{K_{0}} y_{k}^{0}\right)=w^{T} \mu^{0},
$$

where $\mu^{0}$ is the sample mean of the original data. The sample variance for $\bar{y}$ is given by

$$
\begin{aligned}
\bar{\sigma}_{0}^{2} & =\frac{1}{K_{0}} \sum_{k}^{K_{0}}\left(\bar{y}_{k}-\bar{\mu}^{0}\right)^{2}=\frac{1}{K_{0}} \sum_{k}^{K_{0}}\left(w^{T} y_{k}^{0}-\bar{\mu}^{0}\right)^{2}=\frac{1}{K_{0}} \sum_{k}^{K_{0}}\left(w^{T} y_{k}^{0}\right)^{2}-\left(\bar{\mu}^{0}\right)^{2} \\
& =\frac{1}{K_{0}} \sum_{k}^{K_{0}} w^{T}\left(y_{k}^{0} y_{k}^{0^{T}}\right) w-w^{T}\left(\mu \mu^{T}\right) w=w^{T}\left(\frac{1}{K_{0}} \sum_{k}^{K_{0}} y_{k}^{0} y_{k}^{0^{T}}-\mu^{0} \mu^{0^{T}}\right) w \\
& =w^{T}\left(\frac{1}{K_{0}} \sum_{k}^{K_{0}}\left(y_{k}^{0}-\mu^{0}\right)\left(y_{k}^{0}-\mu^{0}\right)^{T}\right) w=w^{T} \Sigma^{0} w .
\end{aligned}
$$

where $\Sigma^{0}$ is the sample covariance matrix. The Fisher linear discriminant chooses $w$ that maximizes the quotient between average class separation and total variation in the transformed domain, this criterion is written as

$$
V(w)=\frac{\left(\bar{\mu}^{f}-\bar{\mu}^{0}\right)^{2}}{\bar{\sigma}_{0}^{2}+\bar{\sigma}_{f}^{2}}=\frac{\left(w^{T}\left(\mu^{f}-\mu^{0}\right)\right)^{2}}{w^{T}\left(\Sigma^{0}+\sum f\right) w}=\frac{w^{T}\left(\mu^{f}-\mu^{0}\right)\left(\mu^{f}-\mu^{0}\right)^{T} w}{w^{T}\left(\Sigma^{0}+\sum f\right) w},
$$

which is a generalized Rayleigh quotient. Defining $\widetilde{\mu} \triangleq \mu^{f}-\mu^{0}$ and $\Sigma \triangleq \Sigma^{0}+\Sigma^{f}$, for a positive definite $\Sigma$, the maximum of $V(w)$ follows from the Cauchy-Schwarz inequality

$$
V(\boldsymbol{w})=\frac{\left(\boldsymbol{w}^{T} \widetilde{\boldsymbol{\mu}}\right)^{2}}{\boldsymbol{w}^{T} \sum \boldsymbol{w}}=\frac{\left(\left(\Sigma^{1 / 2} \boldsymbol{w}\right)^{T}\left(\Sigma^{-1 / 2} \widetilde{\boldsymbol{\mu}}\right)\right)^{2}}{\boldsymbol{w}^{T} \sum \boldsymbol{w}} \leq \frac{\left(\boldsymbol{w}^{T} \sum \boldsymbol{w}\right)\left(\widetilde{\boldsymbol{\mu}}^{T} \Sigma^{-1} \widetilde{\boldsymbol{\mu}}\right)}{\left(\boldsymbol{w}^{T} \sum \boldsymbol{w}\right)}=\widetilde{\boldsymbol{\mu}}^{T} \Sigma^{-1} \widetilde{\boldsymbol{\mu}},
$$

and the bound is attained for the optimum

$$
\boldsymbol{w}^{*}=\Sigma^{-1} \widetilde{\boldsymbol{\mu}}=\left(\Sigma^{0}+\Sigma^{f}\right)^{-1}\left(\boldsymbol{\mu}^{f}-\boldsymbol{\mu}^{0}\right) .
$$

In case $\Sigma$ is positive semi-definite, a regularization term can be added, see, e.g., Friedman (1989).

\subsection{Decision Rule}

Test quantities can be tested jointly or separately to generate symptoms. The choice will depend on the objectives of fault isolation and on how the generated symptoms can relate to faults. For the presentation here, it is considered that each test quantity is tested separately, so that as many symptoms as possible are generated. The presentation is mainly based on the literature of detection theory, see, e.g., Basseville and Nikiforov (1993); Van Trees (2001). 
A test quantity, $q$, is used to test whether the behavior of the extracted features conform to a nominal behavior or not. Each of these conjectures can be seen as a hypothesis. The null hypothesis $\mathcal{H}^{0}$ corresponds to the case where the features and their nominal behavior agree and the alternative hypothesis is $\mathcal{H}^{1}$. Due to the effects of noise and random disturbances, the test quantity will present a random behavior that will differ according to the hypothesis present. This can be modeled by the statistical behavior of the test quantity under the hypotheses,

$$
\mathcal{H}^{0}: q \sim p^{0}(q), \quad \mathcal{H}^{1}: q \sim p^{1}(q) .
$$

A decision rule takes the test quantity as input and chooses either of the hypotheses. A general model for the decision rule is thus $\phi(q): \mathbb{R} \mapsto\{0,1\}$ where $\phi(q)=0$ implies that $\mathcal{H}^{0}$ is accepted, otherwise $\mathcal{H}^{1}$ is chosen. Because only two outcomes are possible, the decision rule can be specified by considering the acceptance region, $\mathcal{R}_{0}$, where $\phi(q)=0$,

$$
\mathcal{R}_{0}=\{q: \phi(q)=0\}
$$

and its complement $\mathcal{R}_{0}^{c}$ is the set giving the alternative outcome $\phi(q)=1$.

Performance of the decision rule can be measured by the probabilities of making erroneous decisions, given by

$$
\begin{aligned}
& P_{f}=\operatorname{Pr}\left[\phi(q)=1 \mid \mathcal{H}^{0} \text { is true }\right]=\int_{\mathcal{R}_{0}^{c}} p^{0}(q) \mathrm{d} q=1-\int_{\mathcal{R}_{0}} p^{0}(q) \mathrm{d} q, \\
& P_{m}=\operatorname{Pr}\left[\phi(q)=0 \mid \mathcal{H}^{1} \text { is true }\right]=\int_{\mathcal{R}_{0}} p^{1}(q) \mathrm{d} q
\end{aligned}
$$

where $P_{f}$ is the probability of false alarm, i.e., deciding incorrectly for $\mathcal{H}^{1}$, and $P_{m}$ is the probability of missed detection, i.e., deciding incorrectly for $\mathcal{H}^{0}$. The complement $1-P_{m}$ is also denoted the probability of (correct) detection, $P_{d}$. In defining the decision function, $\phi(\cdot)$, different objectives are possible but in general low $P_{f}$ and $P_{m}$ are sought.

The Neyman-Pearson criteria attempt to minimize one of the error probabilities while the other is constrained to an upper bound, i.e.

$$
\begin{array}{lcl}
\min _{\mathcal{R}_{0}} P_{m} & \min _{\mathcal{R}_{0}} P_{f} \\
\text { s.t. } P_{f} \leq P_{f}^{\prime}, & \text { or, alternatively, } & \text { s.t. } P_{m} \leq P_{m}^{\prime},
\end{array}
$$

where $P_{f}^{\prime}$ and $P_{m}^{\prime}$ are pre-specified values. In general, the decision errors cannot be made arbitrarily small and a compromise must be made. The next section introduces a simple decision rule. 


\subsubsection{Thresholding}

The simplest and most common decision rule is to consider direct thresholding of the test quantity, in which case the acceptance region is given by

$$
\mathcal{R}_{0}=\{q \mid q \leq \hbar\} .
$$

For a threshold check, the error probabilities can be computed as

$$
P_{f}=\int_{\hbar}^{\infty} p^{0}(q) \mathrm{d} q, \quad P_{m}=\int_{-\infty}^{\hbar} p^{1}(q) \mathrm{d} q,
$$

and the choice of threshold $\hbar$ can be motivated from a Neyman-Pearson criterion or chosen based on available knowledge of the behavior of the test quantity. Example 4.7 illustrates the use of thresholding for a test quantity used to detect wear changes in a robot joint and illustrates some of the compromises in the threshold selection.

\section{Example 4.7: Detection of wear changes in a robot joint}

The test quantity defined in (4.23) is considered for torque data collected from a robot joint under accelerated wear tests as in Example 4.6. Given two batches of torque data $\tau^{m}$ and $\tau^{n}$, the test quantity is $q=\mathrm{KL}\left(\widehat{p}^{m}, \widehat{p}^{n}\right)$ where $\widehat{p}^{m}(\tau)$ and $\widehat{p}^{n}(\tau)$ are kernel density estimates from the respective torque batches.

As presented in Example 4.2, the available torque data are affect by the wear level in the joint, $\omega$, as well as the temperature in the joint, $\xi$, i.e., $\boldsymbol{\tau}(\omega, \xi)$. Temperature is considered as a disturbance since it is not measured. Based on simulations, the torque sequences are generated under the two setups (hypotheses)

$$
\begin{array}{ll}
\mathcal{H}^{0}: & \boldsymbol{\tau}^{m}(\omega=0, T) \text { and } \boldsymbol{\tau}^{n}(\omega=0, T), \\
\mathcal{H}^{1}: & \boldsymbol{\tau}^{m}(\omega=0, T) \text { and } \boldsymbol{\tau}^{n}\left(\omega=\omega_{c}, T\right),
\end{array}
$$

i.e., no wear effects are present for $\mathcal{H}^{0}$ and a critical wear increase of size $\omega_{c}=35$ is present for $\mathcal{H}^{1}$. For analysis of the effects of temperature disturbances, $\xi$ is considered random with a uniform distribution

$$
\xi \sim \mathcal{U}\left(\underline{\xi}, \underline{\xi}+\Delta_{\xi}\right),
$$

where $\underline{\xi}=30^{\circ} \mathrm{C}$ and the value of $\Delta_{\xi}$ relates to the spread of the disturbance. The distributions of the test quantity under each hypothesis, $p\left(q \mid \mathcal{H}^{0}\right)$ and $p\left(q \mid \mathcal{H}^{1}\right)$, are estimated using a kernel density estimator based on Monte Carlo simulations. They are shown for different levels of temperature disturbances $\Delta_{\xi}$ in Figures $4.8 \mathrm{a}$ to $4.8 \mathrm{c}$. As can be seen, the overlap between the hypotheses densities increases with $\Delta_{\xi}$, complicating a decision. The probabilities of error are shown in Figure $4.8 \mathrm{~d}$ for different values of the threshold, illustrating that performance can considerably vary depending on the disturbances for any given threshold choice. 


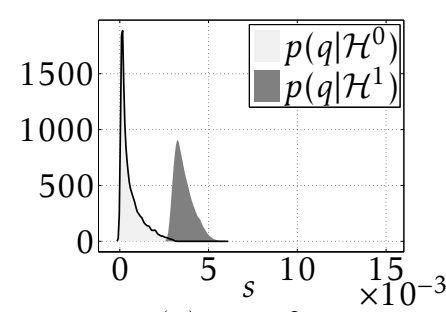

(a) $\Delta_{\xi}=6^{\circ} \mathrm{C}$.

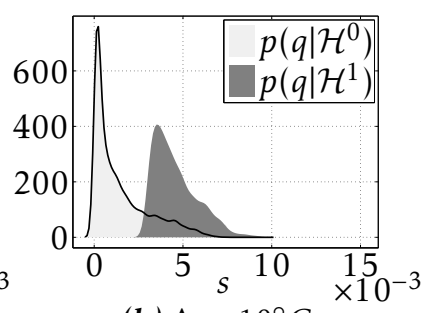

(b) $\Delta_{\xi}=10^{\circ} \mathrm{C}$.

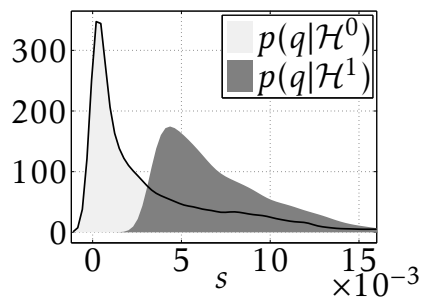

(c) $\Delta_{\xi}=20^{\circ} \mathrm{C}$.

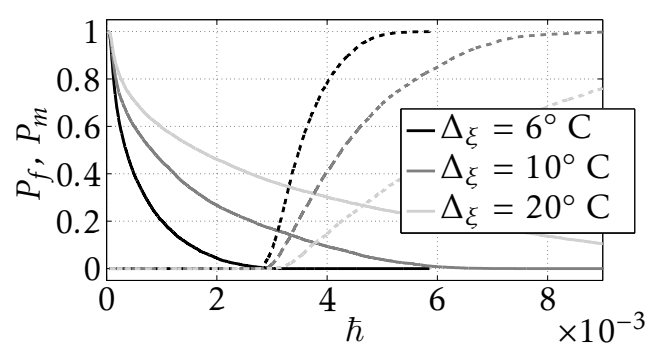

(d) $P_{f}$ (solid) and $P_{m}$ (dashed).

Figure 4.8: Kernel density estimates for the hypotheses' densities under different levels of temperature disturbance $\Delta_{\xi}$ (top row). The probabilities of error as a function of the threshold $\hbar$ (bottom).

\subsubsection{Likelihood ratio tests}

As depicted by (4.27), the performance for a decision rule is determined by the statistical behavior of the test quantity under the different hypotheses, described by (4.25). In case these models are known, it is thus natural to consider them when defining the decision rule. In this direction, the following result is fundamental.

Lemma 4.1 (Neyman-Pearson). Consider the hypotheses given by (4.25). Let the likelihood ratio function be defined as

$$
\Lambda(q) \triangleq \frac{p^{1}(q)}{p^{0}(q)} .
$$

For $\hbar \geq 0$, define the acceptance region

$$
\mathcal{R}_{0}=\{\Lambda(q) \leq \hbar\},
$$

with error probabilities

$$
P_{f}=\int_{\hbar}^{\infty} p\left(\Lambda \mid \mathcal{H}^{0}\right) \mathrm{d} \Lambda, \quad P_{m}=\int_{-\infty}^{\hbar} p\left(\Lambda \mid \mathcal{H}^{1}\right) \mathrm{d} \Lambda .
$$

Let another acceptance region $\mathcal{R}_{0}^{\prime}$ with error probabilities $P_{f}^{\prime}$ and $P_{m}^{\prime}$. If $P_{f}^{\prime} \leq P_{f}$ 
then $P_{m}^{\prime} \geq P_{m}$. Additionally, if $P_{m}^{\prime} \leq P_{m}$ then $P_{f}^{\prime} \geq P_{f}$.

Proof: See Theorem 11.7.1 in Cover and Thomas (2006).

The test based on the likelihood ratio in (4.29) is therefore optimal according to to a Neyman-Pearson criterion. Notice that the threshold $\hbar$ can be found for a Neyman-Pearson criterion by solving either of the equations in (4.30) for a prespecified left hand side.

\section{Generalized likelihood ratio tests}

In case the hypotheses densities are described by unknown parameters,

$$
\mathcal{H}^{0}: q \sim p\left(q \mid \boldsymbol{\theta}^{0}\right), \quad \mathcal{H}^{1}: q \sim p\left(q \mid \boldsymbol{\theta}^{1}\right),
$$

there is not a general test that is optimal in a Neyman-Pearson sense. A natural approximation is to first find the maximum likelihood estimate of the parameters and use the resulting likelihood ratio, i.e.,

$$
\widehat{\Lambda}(q)=\frac{\max ^{1} p^{1}\left(q \mid \theta^{1}\right)}{\max _{\boldsymbol{\theta}^{0}} p^{0}\left(q \mid \theta^{0}\right)} .
$$

The test with acceptance region given by $\mathcal{R}_{0}=\{\widehat{\Lambda}(q) \leq \hbar\}$ is called a generalized likelihood ratio test (GLR). Asymptotic optimality conditions (when the number of observations of the test quantity goes to infinity) of the generalized likelihood ratio test are studied in Zeitouni et al. (1992).

\subsubsection{Statistical significance tests}

In order to evaluate likelihood ratio tests, the distribution functions $p^{1}(q)$ and $p^{0}(q)$ (or their parametric description) must be known. In practice, this is often not possible, particularly for the alternative hypothesis $\mathcal{H}^{1}$ which describes abnormal behaviors which are typically unknown.

It is possible to define an acceptance region, $\mathcal{R}_{0}$, based only on a model for the null hypothesis $\mathcal{H}^{0}$. For a pre-defined $P_{f}^{\prime}$, the acceptance region can be found as the solution to

$$
P_{f}^{\prime}=1-\int_{\mathcal{R}_{0}} p^{0}(q) \mathrm{d} q .
$$

The resulting acceptance region tests whether the observations are consistent to its nominal behavior. There is not a unique $\mathcal{R}_{0}$ that satisfies this criterion. For example, a decision region defined over the entire space except for a value $q^{*}$, i.e., $\mathcal{R}_{0}=\mathbb{R}-q^{*}$, gives arbitrarily small $P_{f}$ for any choice of $q^{*}$. It is common to consider $\mathcal{R}_{0}$ as the smallest continuous interval in the observation space, when the decision region is known as the confidence interval. This type of decision rule was used in Example 4.4 to test for significant changes in parameter values. 


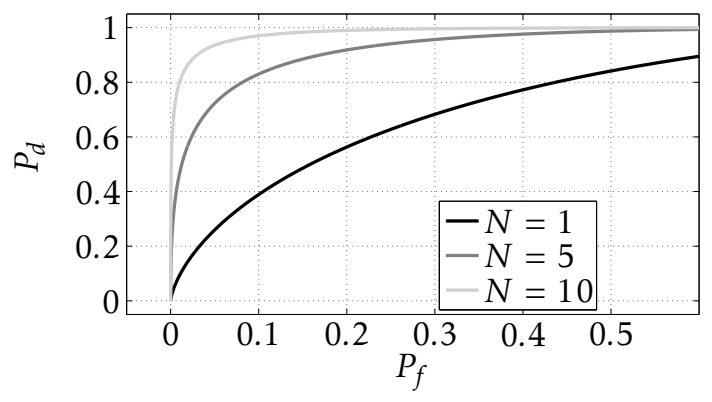

Figure 4.9: $R O C$ curves illustrating the compromise between detection error and sample size (detection time).

\subsubsection{Compromises between errors and time of detection}

Even optimal likelihood ratio tests may not achieve the performance requirements for a given application. The performance can be improved if it is possible to consider more data before making a decision. This is more easily described with an example.

Example 4.8: Likelihood ratio test for a change in the mean of a Gaussian $\neg$ Consider that $N$ observations of the test quantity are available to perform the decision, i.e., $\boldsymbol{q} \in \mathbb{R}^{N}$. As an example, take the following hypotheses

$$
\mathcal{H}^{0}: \boldsymbol{q} \sim \mathcal{N}(\mathbf{0}, I), \quad \mathcal{H}^{1}: \boldsymbol{q} \sim \mathcal{N}(\mathbf{1}, I),
$$

the acceptance region given by the likelihood ratio for this problem is

$$
\Lambda(\boldsymbol{q})=\frac{|2 \pi I|^{-1 / 2} e^{-\frac{1}{2}(\boldsymbol{q}-\mathbf{1})^{T}(\boldsymbol{q}-\mathbf{1})}}{|2 \pi I|^{-1 / 2} e^{-\frac{1}{2} \boldsymbol{q}^{T} \boldsymbol{q}}}=e^{-\frac{1}{2}\left(-2 \boldsymbol{q}^{T} \mathbf{1}+N\right)} \leq \hbar .
$$

Taking the logarithm (a monotonic transformation) does not affect the inequality and isolating $q$ on the left hand side gives the equivalent acceptance region

$$
s \triangleq \sum_{n=1}^{N} q_{n} \leq \hbar^{\prime}
$$

for a new threshold $\hbar^{\prime}$. The quantity $s$ is a sufficient statistic for the test since it describes it completely. According to (4.31), the distribution of $s$ under the different hypotheses are

$$
p\left(s \mid \mathcal{H}^{0}\right)=\mathcal{N}(s ; 0, N), \quad p\left(s \mid \mathcal{H}^{1}\right)=\mathcal{N}(s ; N, N)
$$

and the probabilities of error are

$$
P_{f}=\int_{\hbar^{\prime}}^{\infty} \frac{1}{\sqrt{2 \pi N}} e^{-\frac{s^{2}}{2 N}} \mathrm{~d} s, \quad P_{m}=1-P_{d}=1-\int_{\hbar^{\prime}}^{\infty} \frac{1}{\sqrt{2 \pi N}} e^{-\frac{(s-N)^{2}}{2 N}} \mathrm{~d} s
$$

which are functions of the number of observations $N$. By varying the threshold 
$\hbar^{\prime}$ from $-\infty$ to $\infty$ and plotting $P_{f}$ against $P_{d}$, a receiver operating characteristic (ROC) curve is achieved and can be used to evaluate decision rules. ROC curves are displayed for different values of $N$ in Figure 4.9 where the effects of the sample size is clear.

As illustrated by the example, increasing the number of observations can lead to an improved detection performance. The shortcoming is that an accurate enough decision will be postponed before enough data is collected. That is, an accurate enough decision is compromised with the detection time.

Heuristic approaches can also be used to improve the detection errors. For instance, the test quantity can be low pass filtered before input to the decision rule or a decision can be made after the test quantity exceeds the threshold for a number of times consecutively. These will however introduce delay to the detection (Adnan et al., 2011).

\subsection{Summary and Connections}

This chapter presented an overview of the diagnosis process. The presentation focused on describing different fault detection methods, with special attention to methods that are suitable to the main application considered, i.e., detecting friction and wear changes in industrial robots. A number of examples were presented to illustrate relevant methods and trade-offs.

Gathering knowledge about the disturbances and faults is an important aspect for the design and verification of fault detection methods. The achieved friction models from Papers A and B allow for a more realistic design and evaluation of solutions to wear diagnosis in industrial robot joints. These models were used in Example 4.7.

An off-line active method for wear monitoring is proposed in Paper B. The method is based on the maximum likelihood estimation of a wear related quantity and makes use of the developed friction models and a dedicated test-cycle. Experiment design is considered in detail as to achieve as accurate estimate of wear as possible for a limited experiment time.

In Paper $\mathrm{C}$, distribution domain methods are proposed for fault detection and isolation of repetitive systems. Changes in the distribution of data collected from a repetitive operation are monitored by test quantities similar to that of (4.23). In order to reduce sensitivity of the test quantities to disturbances, an approach is suggested based on the use of a weighting function which is found based on a criterion closely related to the Fisher linear discriminant in (4.23). Improvements in detection performance achieved with the use of the weights is illustrated based on simulations, in a similar approach as presented in Example 4.7.

The measures of performance defined in Section 4.3 are also used in Paper D when defining an approach to simulation based evaluation and comparison of fault detection algorithms. 
Paper E proposes studies the design of decision rules that only require nominal data of the test quantity. The approach is divided in two steps. First, a nonparametric density model for the nominal behavior of the test quantity, $\widehat{p}^{0}(q)$ is found based on the available data. Second, generalized likelihood ratio tests are defined based on the assumption that the alternative hypothesis is described by $\widehat{p}^{0}(q \mid \Delta)=\widehat{p}^{0}(q-\Delta)$, i.e., under the assumption that a fault will appear as a bias change. The unknown change size $\Delta$ is found based on maximum likelihood estimates. 



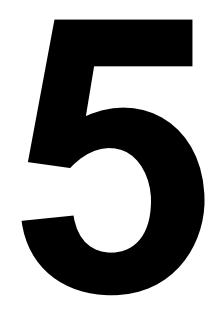

\section{Conclusions and Discussion}

The first part provided an introduction to the research fields that are relevant for this thesis: industrial robotics, tribology and diagnosis. This served as a preparation to Part II, motivating the research contributions and contextualizing them. The conclusions for Part I that relate to the research goals of the thesis are given in Section 5.1. The discussion is followed by a summary of the research contributions of Part II, overall conclusions of the thesis in Section 5.3 and recommendations of future research in Section 5.4. See also the included papers for details.

\subsection{Conclusions of Part I}

As presented in Chapter 2, there are different aspects of industrial robotics that challenge the research goals of this thesis.

Complex dynamics. Industrial robots are nonlinear, multi-variable, uncertain systems operating in closed-loop.

Limited sensory information. In a typical setup, only motor angular positions $\varphi^{m}$, and applied motor currents $\boldsymbol{i}^{m}$ are measured. From these measurements, estimates of angular speeds $\dot{\varphi}^{m}$ and the applied motor torques $\tau^{m}$ are possible.

Application-related limitations. Industrial robots are used in a wide range of applications. Depending on the installation, there will be restrictions on the available workspace and on how the robot can be used.

For various reasons, the use of models is important in industrial robotics. Due to the complexity of a robot joint, models describing, e.g., backlash and friction are difficult and are often motivated from empirical observations. With interest 
in the determination of critical wear changes based on its effect to friction, Chapter 3 presented an overview of friction in a robot joint. Important characteristics of friction and wear that should be considered when designing solutions for diagnosis are:

Small fault to disturbance ratio. The effects of temperature and load variations to friction are comparable, often larger, than the effects related to wear.

Complex and individual behavior. The friction behavior is determined by complex interactions at a surface level. As a result, although a similar qualitative behavior may be observed for different gearbox-motor pairs, its quantitative behavior will differ in general.

Lubricant. The use of different lubricants will considerably affect friction as well as its temperature dependencies. It is thus important to keep track of possible changes of lubricant and its condition.

Unpredictability of the wear processes. The effects of wear to friction will depend on how the wear takes place in the mechanical parts of the joint which are in general difficult to predict and determine.

Chapter 4 gave an overview of fault diagnosis and some of the challenges involved. In fault diagnosis, observations and available knowledge of the system are compared to infer the state present in the system. The knowledge embedded in the diagnosis solution can take different forms, e.g., a system model or expert knowledge of signals and data. The type of knowledge representation available will affect the design and application requirements. Since only limited knowledge can be embedded in a diagnosis solution, the following factors are important to consider.

Evaluation. Methods should be evaluated extensively based on real data as much as possible and complemented with realistic laboratory and simulation studies.

Detection compromises. A correct determination of fault presence cannot be performed with arbitrary accuracy and promptness and compromise must be made.

All of these aspects will delimit the applicability of possible solutions and should therefore be considered before deployment.

\subsection{Summary and Discussion for Part II}

\section{Paper A: Friction in a Robot Joint - Modeling and Identification of Load and Temperature Effects}

In Paper A, a detailed empirical study of friction in a robot joint is presented. The study was motivated by the complexity of friction in a robot (recall Figure 3.2). In the paper, the typical friction related phenomena and models used in robotics are reviewed. The effects of angular position, speed, load torques and 
temperature to friction in a robot joint are considered. Due to their relevance, a model to describe the effects of speed, load and temperature is suggested and validated. The proposed model considerably outperforms standard friction models and is important for design and evaluation of diagnosis methods but also for control and simulation. The requirements for the identification of the complete friction model are described below, together with a description of the suggested approaches for their accomplishment.

Rich friction data. Friction data collected under large variations of load, temperature and speed are needed in order to accurately find the parameters.

A simple and short experiment is suggested for the collection of a friction datum at a given speed value. The experiment can be repeated for different temperature, load and speed conditions until enough data are collected to accurately estimate the parameters.

As suggested in the paper, variations of load can be achieved by performing the experiment in different configurations of the robot but will require a large region of the robot workspace. Variations of temperature are more difficult since the thermal constant in a robot joint is of several hours.

Temperature and load estimates. Estimates or measurements of the joint temperature and of load torques are needed in order to find the associated friction parameters.

An estimate for the load torques is possible based on a robot model. In the experiments, temperature was measured with a temperature probe immersed in the gearbox lubricant oil.

The requirements for the identification procedure will possibly exceed the constraints posed by the robot user since extra sensory information, a large region of the workspace and a long experiment time are needed. For these reasons, the procedure is better suited for laboratory studies. For instance, the model can be found during commissioning of newly produced robots.

It is worth mentioning that alternatives to lubricant temperature measurements are possible. For instance, it is possible to find an estimate based on availability of environment and housing temperature sensors and a thermal model for the joint as described by Marton and van der Linden (2012). However, in order to find the thermal model for the joint, readings of the lubricant temperature are also needed.

\section{Paper B: Modeling and Experiment Design for Identification of Wear in a Robot Joint Under Load and Temperature Uncertainties}

In Paper B, the effects of wear to friction are modeled and identified. The model is based on empirical studies from accelerated wear tests performed in a robot joint. This model is combined with the friction model suggested in Paper A to achieve a description of friction as function of speed, temperature, load and wear. Using the resulting model, a method is proposed for wear monitoring. The method 
is based on the estimation of a wear related quantity from friction data and is formulated as a maximum likelihood problem.

To study the applicability of parameter estimation as a solution for wear monitoring, a simplified but realistic scenario is considered with availability of a friction model and friction data. These are the requirements for the method and, as suggested in the paper, they can be achieved as follows.

Estimates of load torques. It is considered that an uncertain estimate of the load torques at the joints is available. This can be achieved with the use of a robot model.

Friction model parameters related to temperature and load. These parameters describe the nominal behavior of friction and can be found based on laboratory experiments, as suggested for Paper A.

Friction model parameters related to the effects of wear. The effects of wear to friction will depend on the behavior of the fault, which can not be known a priori. To circumvent this, it is suggested that these effects are studied and modeled based on historical failure data, e.g., collected from accelerated wear tests. From such historical data, parameters can be found for the model structure suggested in the paper or, if needed, an alternative model structure can be developed that is better suited for the observed phenomena.

Friction data. The same experiment suggested for Paper A to collect a friction datum for a certain speed level can be used.

Imposed by the total allowed experiment time, there will be a fixed number of friction data points that can be be collected. To achieve as accurate as possible estimate of the wear quantity, a criterion for experiment design is used to select the speed values that will lead to a reduced uncertainty for the wear estimate. More accurately, the criterion aims at minimizing the Cramér-Rao lower bound for the covariance of unbiased wear estimators.

The approach is validated with special attention to limitations imposed by temperature and load. As it is shown from simulations and real data studies, for an experiment time constrained to less than one minute for the entire robot, it is possible to accurately estimate the wear quantity despite variations of load and temperature. This was possible even in scenarios where the parameters related to a wear fault were not optimized for the faults taking place. An important advantage with the approach is that the estimated wear parameter directly relates to effects of wear to friction and therefore allows for a natural interpretation.

\section{Paper C: A Data-driven Approach to Diagnostics of Repetitive Processes in the Distribution Domain}

In Paper C, data-driven methods are proposed for diagnosis of systems that behave in a repetitive manner. The basic idea is to compare data collected in different instances of time from a repetitive behavior to provide an estimate of how 
the system changed over the period.

A data-driven method is suggested where the distribution of incoming data is compared to the distribution of data collected under nominal conditions. A kernel density estimator is used, in combination with the Kullback-Leibler distance. An approach is also suggested to reduce sensitivity to disturbances with the use of a weighting function. The requirements for the methods are given below.

Data from a repetitive operation. A repetitive operation is commonly found in many applications, e,g, for industrial robots and in automated manufacturing. A repetitive behavior can also be forced with the execution of a diagnostic routine.

Nominal data. Data collected from the system under nominal conditions are needed. For example, these data can be collected from the system when it is new.

Weighting function (optional). As suggested in the paper, data can be weighted according to a weighting function to reduce sensitivity to disturbances.

A procedure is suggested to find the weights based on a criterion similar to that found for Fisher discriminant analysis. The procedure requires availability of fault-free as well as faulty data. In the paper, such data are achieved from simulations.

The methods are illustrated for the problems of wear monitoring in industrial robots and for the diagnosis of gear faults in a gearbox system. For the robotics problem, the effects of temperature are studied based on simulations, where it is shown that the use of the weighting function can considerably reduce sensitivity to temperature variations. An important advantage of the approach is that no models are needed and it is also simple to implement.

\section{Paper D: Simulation based Evaluation of Fault Detection Algorithms}

In Paper D, a framework is suggested for the evaluation of fault detection algorithms. The effects of faults and disturbances to the test quantities are studied and approaches are proposed in an attempt to determine,

i) which disturbances affect a method the most,

ii) which methods perform better in average to indicate presence of a fault,

iii) which combinations of fault change size and variations of disturbances are allowed to achieve satisfactory performance.

A simulation based approach is suggested since it allows for a more comprehensive study than it is often possible based on real data. This is particularly critical for studies of wear faults in industrial robots since wear tests are both cost and time critical.

Even with simulation data, some studies may take prohibitive amounts of time. An important idea suggested in the paper is to bypass the need for data by mak- 
ing a direct map from faults and disturbances to the test quantity. Such direct map is known as a surrogate model and includes both the simulator and fault detection algorithm. The advantage is that, once the surrogate models are found and validated, they can be used to calculate the response of the test quantities for different values of faults and disturbances in a very short time, thus allowing for extensive Monte Carlo simulations. For example, one of the studies in the paper was evaluated in twelve seconds with the surrogate models. The same study performed based on simulations of the entire system would have taken more than three years.

The use of the proposed approaches is illustrated for the problem of wear monitoring in industrial robots where three fault detection algorithms are evaluated. The study reveals interesting properties of the problem and of the candidate methods. For instance, it shows that variations of temperature significantly affect all methods considered. It also shows how much variations of load and temperature are allowed to clearly detect a wear fault of a certain size, revealing the scope of their applicability. Finally, it also gives a criterion for selection of the fault detection algorithm that performs best in average.

It should be stressed that conclusions drawn based on simulations or surrogate models should always be carried out carefully since these are a limited representation of reality. Nevertheless, this type of study can reveal valuable properties of the problem at hand and of the fault detection methods. For instance, they can be used to justify choices regarding further developments and selection of candidate solutions.

\section{Paper E: Data-Driven Anomaly Detection based on a Bias Change}

Paper E considers the problem of determination of a decision rule which,

i) requires minimal and meaningful specification parameters from the user,

ii) is flexible and can be used for different problems,

iii) can provide estimates for the probabilities of error in the decision.

The suggested approach only requires availability of nominal data of the test quantity, which is a common situation in practice. The user only needs to specify a desired probability of false detection, which is a natural design choice. No model or other parameters need to be pre-determined.

The suggested decision rule is achieved in two steps based on approximate models for the statistical behavior of the test quantity. First, the nominal data are used to find a density model to describe the nominal behavior (the null hypothesis) of the test quantity, $\widehat{p}^{0}(q)$. Non-parametric density models are used, giving the required flexibility.

In order to provide estimates of the probability of miss as in (4.27b), a statistical model for the behavior of the test quantity under presence of a change (the alternative hypothesis) is needed. An assumption is introduced that presumes that 
an abnormality will affect the test quantity as a bias change relative to its nominal statistical behavior, i.e., $\widehat{p}^{1}(q)=\widehat{p}^{0}(q-\Delta)$, which is a simple and intuitive model. The unknown change size $\Delta$ is estimated from incoming test data based on suggested maximum likelihood estimators.

The resulting models $\widehat{p}^{0}(q)$ and $\widehat{p}^{1}(q)=\widehat{p}^{0}(q-\widehat{\Delta})$ are then used to define generalized likelihood ratio tests assuming that these are the true models. Batch and sequential approaches are suggested which are important depending on the application sought. The proposed decision rule is illustrated for real data problems as well as simulations. When applied to the wear monitoring problem, it is shown how an early detection can be made with the suggested decision rule. It also provides estimates of the decision uncertainties, which are useful to support higher level service decisions.

\subsection{Conclusions}

Returning to the main research goals of the thesis,

Design and investigate the applicability of methods to detect critical changes of wear based on standard sensory information and limited intervention with the system operation to support service.

Considering the achieved results, the following conclusions are made.

Understanding. The studies and modeling of friction and wear presented in $\mathrm{Pa}$ pers $\mathrm{A}$ and $\mathrm{B}$ contribute to the overall understanding of the phenomena. Without these studies it would have been difficult to verify the applicability of the proposed solutions. Beyond the examples reported in the thesis, similar phenomena were observed for several robot units from studies carried out in field, laboratory and production environments, increasing the confidence in the generalization of the studies.

Design. Methods for wear monitoring were suggested in Paper B based on the identification of a wear quantity and in Paper $C$ based on a data-driven approach. Both are based on standard sensory information but have different requirements as discussed in the previous section.

The method based on the estimation of a wear parameter requires availability of a friction model as well as friction data which can be collected based on an experiment that takes one minute or less. The data-driven approach does not require a friction model and is very simple to implement but requires nominal data and the achieved test quantity does not carry a natural interpretation as for the wear estimates. In case a repetitive operation can be found during normal operation, the data-driven approach does not require dedicated experiments. Otherwise, repetitive data can be collected based on an experiment of similar length as for the wear estimation.

Applicability. The suggested methods for wear monitoring were evaluated with success based on real data as well as simulations in realistic scenarios in 
Papers B and C. The simulation based evaluation framework suggested in Paper D can also serve as a tool to investigate the applicability of the methods.

The methods were also successfully evaluated in a number of additional accelerated wear tests, increasing confidence in their applicability. However, it would be advisable to extend the verification based on more studies carried out in the field.

Furthermore, because the quantitative behavior of friction in a tribological system is rather individual, changes affecting it, such as the replacement of the lubricant or components in the joint need to be considered as they can affect the methods. For instance, they may require an update of the parameters for the friction model or the assignment of new nominal data.

Tools to support service. The methods for wear monitoring proposed in Papers $\mathrm{B}$ and $\mathrm{C}$ are important tools to support the service of industrial robots. The methods presented in Paper E also provide the service engineer with tools for an automatic determination of fault presence. The evaluation framework of Paper D provides valuable tools to support the design, evaluation and comparison of different methods.

The research goals of this thesis have therefore been addressed in multiple fronts.

\subsection{Recommendations for Future Research}

Several further developments are suggested in the end of each paper in Part II. Besides those, the following aspects are emphasized.

Understanding and modeling of friction. More extensive studies of friction will support further design and evaluation of possible solutions.

- Modeling of dynamic friction. Only static friction models were developed in this work. One possibility is to verify whether the static friction models developed can be extended to a dynamic description, e.g., based on the LuGre model.

- Validation over larger temperature ranges. The temperature effects were modeled in a temperature range of $35-80^{\circ} \mathrm{C}$. Robots may operate outside this range. It is therefore important to understand how friction would be affected.

- Verification of the developed models in other mechanical devices. Realistic friction models are important in many applications. The effects of load and temperature are often neglected, despite their importance. In principle, the developed models can be extended to other types of mechanical devices under lubricated friction. The use of the model structure proposed would simplify the time consuming task of developing new friction models. 
For instance, while the study of friction considered here focused on the main axes of the robot, it would be interesting to study friction also in the wrist axes.

Understanding and modeling of wear. Since it is costly and time consuming to perform wear experiments, wear models are very important for the design and verification of diagnosis solutions. The further study of wear and wear modeling in a robot joint is motivated by the following.

- Unpredictability of the wear processes. It is not possible to assure that a wear model developed based on an observed fault is representative of future faults. The wear processes in a joint are the results of complex phenomena that cannot be easily predicted. With more knowledge gathered about the faults, it is possible to describe them in a more detailed manner. Perhaps different wear models can be developed to describe the most common faulty behaviors. This would be useful for instance for the wear estimation method proposed in Paper B.

- Development of lifetime models. Understanding how wear evolves with time and usage is important for the design and verification of diagnosis solutions. For example, it supports the scheduling of diagnostic tests and the design of decision rules.

A lifetime model also allows for prognosis, which is very important for the scheduling of maintenance routines.

Design of alternative solutions. It would be interesting to investigate alternative solutions for wear monitoring with reduced requirements and/or improved accuracy and reliability.

- Monitoring based on operational data. The methods suggested in the thesis are, in general terms, better suited for the scenario where it is acceptable to perform dedicated experiments for diagnosis. Although these experiments are short in time and in the required use of the workspace, it would be interesting to further investigate methods where no dedicated experiments are needed. The applicability of such methods will however depend on how the system is being used. Furthermore, additional uncertainties may also appear, e.g., related to unmodeled flexible dynamics of the robot, and the evaluation of such methods in realistic scenarios is thus difficult.

Evaluation of the methods. It is important to thoroughly evaluate the approaches before use in real world applications. This will help to identify weaknesses and will provide leads to possible improvements. Three approaches are listed.

- Simulation studies. With the use of the developed friction models, it is possible to setup more realistic simulation models. In simulation studies, it is important to consider the different sources of uncertainties present in practice, e.g., flexibilities, torque ripple, temperature and load variations, closed-loop effects, etc. For on-line methods, trajectories that are used in real world applications should be considered. 
To allow for a comparison of different methods, a benchmark problem for robust wear diagnosis in a robot joint could be defined using such a model.

- Accelerated wear tests. Even though a realistic simulation model is important, it cannot substitute the validation through experiments. Since the wear effects may take several years to occur, accelerated wear tests, performed in a lab, can be used as a first verification. It is however difficult to reproduce scenarios that are representative for what happens in the field. For example, it is difficult to control temperature in the joints.

- Field tests. These are irreplaceable for the evaluation of diagnosis solutions. In order to be of significance, they must be verified with several robots and in different applications. This is however only possible in cooperation with robot users and can take considerable time. 


\section{Bibliography}

N. Adnan, I. Izadi, and T. Chen. Computing detection delays in industrial alarm systems. In Proceedings of the 2011 American Control Conference, pages 786 -791, July 2011.

D. Agarwal. Detecting anomalies in cross-classified streams: a Bayesian approach. Knowledge and Information Systems, 11(1):29-44, 2007.

F. Al-Bender and J. Swevers. Characterization of friction force dynamics. IEEE Control Systems Magazine, 28(6):64-81, 2008.

T. Ardeshiri, M. Norrlöf, J. Löfberg, and A. Hansson. Convex optimization approach for time-optimal path tracking of robots with speed dependent constraints. In Proceedings of 18th IFAC World Congress, pages 14648-14653, Milano, Italy, September 2011.

K. J. Åström and C. Canudas-de Wit. Revisiting the LuGre friction model. IEEE Control Systems Magazine, 28(6):101-114, December 2008.

P. Axelsson. Sensor Fusion and Control Applied to Industrial Manipulators. Linköping Studies in Science and Technology. Dissertations No. 1585, Linköping University, SE-581 83 Linköping, Sweden, May 2014.

M. Basseville. Distance measures for signal processing and pattern recognition. Signal Processing, 18(4):349-369, 1989.

M. Basseville and I. V. Nikiforov. Detection of abrupt changes: theory and application. Prentice-Hall, Inc., Upper Saddle River, NJ, USA, 1993.

A. C. Bittencourt and P. Axelsson. Modeling and experiment design for identification of wear in a robot joint under load and temperature uncertainties based on friction data. IEEE/ASME Transactions on Mechatronics, 19(5):1694-1706, October 2014.

A. C. Bittencourt and S. Gunnarsson. Static friction in a robot joint- Modeling and identification of load and temperature effects. Journal of Dynamic Systems, Measurement, and Control, 134(5), July 2012. 
A. C. Bittencourt and T. Schön. Data-driven anomaly detection based on a bias change. In Proceedings of the 19th IFAC World Congress, Cape Town, South Africa, 2014.

A. C. Bittencourt, K. Saarinen, S. Sander-Tavallaey, S. Gunnarsson, and M. Norrlöf. A data-driven approach to diagnostics of repetitive processes in the distribution domain - Applications to gearbox diagnostics in industrial robots and rotating machines. Mechatronics, -(0):-, 2014. available online.

P. J. Blau. Embedding wear models into friction models. Tribology Letters, 34(1), April 2009.

L. C. Bo and D. Pavelescu. The friction-speed relation and its influence on the critical velocity of stick-slip motion. Wear, 82(3):277-289, 1982.

B. Bona and M. Indri. Friction compensation in robotics: an overview. In Proceedings of the 44th IEEE International Conference on Decision and Control, Seville, Spain, December 2005.

A. W. Bowman and A. Azzalini. Applied Smoothing Techniques for Data Analysis: The Kernel Approach with S-Plus Illustrations (Oxford Statistical Science Series). Oxford University Press, USA, November 1997.

D. Brambilla, L. Capisani, A. Ferrara, and P. Pisu. Fault detection for robot manipulators via second-order sliding modes. IEEE Transactions on Industrial Electronics, 55(11):3954-3963, November 2008.

T. Brogårdh. Present and future robot control development - an industrial perspective. Annual Reviews in Control, 31:69-79, 2007.

T. Brogårdh. Robot Control Overview: An Industrial Perspective. Modeling, Identification and Control, 30(3):167-180, 2009.

M. D. Bryant. Entropy and dissipative processes of friction and wear. FME Transactions, 37(2):55-60, 2009.

F. Caccavale, P. Cilibrizzi, F. Pierri, and L. Villani. Actuators fault diagnosis for robot manipulators with uncertain model. Control Engineering Practice, 17(1): 146-157, 2009.

T. Cacoullos. Estimation of a multivariate density. Annals of the Institute of Statistical Mathematics, 18:179-189, 1966.

M.-O. Cordier, P. Dague, F. Levy, J. Montmain, M. Staroswiecki, and L. TraveMassuyes. Conflicts versus analytical redundancy relations: a comparative analysis of the model based diagnosis approach from the artificial intelligence and automatic control perspectives. IEEE Transactions on Systems, Man, and Cybernetics, Part B: Cybernetics, 34(5):2163-2177, Oct 2004. ISSN 1083-4419. doi: 10.1109/TSMCB.2004.835010.

T. M. Cover and J. A. Thomas. Elements of information theory. Wileyinterscience, 2006. 
J. De Kleer and B. C. Williams. Diagnosing multiple faults. Artificial intelligence, 32(1):97-130, 1987.

C. W. de Silva. Vibration Monitoring, Testing and Instrumentation. CRC Press, April 2007.

M. Desforges, P. Jacob, and A. Ball. Fault detection in rotating machinery using kernel-based probability density estimation. International Journal of Systems Science, 31(11):1411-1426, 2000.

S. X. Ding. Model-Based Fault-Diagnosis Techniques - Design Schemes, Algorithms and Tools. Springer, 2nd edition, 2013.

D. Dowson. History of Tribology. Professional Engineering Publishing, London, UK., 2nd edition, 1998.

P. Dupont, V. Hayward, B. Armstrong, and F. Altpeter. Single state elastoplastic friction models. IEEE Transactions on Automatic Control, 47(5):787 -792, May 2002.

R. Durrett. Probability: Theory and examples. Cambridge University Press, August 2010.

P. Frank and X. Ding. Survey of robust residual generation and evaluation methods in observer-based fault detection systems. Journal of Process Control, 7(6): 403-424, 1997.

B. Freyermuth. An approach to model based fault diagnosis of industrial robots. In Proceedings of the 1991 IEEE International Conference on Robotics and Automation, volume 2, pages 1350-1356, April 1991.

J. H. Friedman. Regularized discriminant analysis. Journal of the American statistical association, 84(405):165-175, 1989.

M. Gautier and P. Poignet. Extended Kalman filtering and weighted least squares dynamic identification of robot. Control Engineering Practice, 9(12):1361 1372, 2001.

A. Gogoussis and M. Donath. Coulomb friction effects on the dynamics of bearings and transmissions in precision robot mechanisms. In Proceedings of the 1998 IEEE International Conference on Robotics and Automation, pages 14401446, Leuven, Belgium, April 1988.

Y. Guo, Z. Qu, Y. Braiman, Z. Zhang, and J. Barhen. Nanotribology and nanoscale friction. IEEE Control Systems Magazine, 28(6):92-100, December 2008.

F. Gustafsson. Adaptive Filtering and Change Detection. Wiley, October 2000.

P. Hamon, M. Gautier, and P. Garrec. Dynamic identification of robots with a dry friction model depending on load and velocity. In Proceedings of the 2010 IEEE/RSJ International Conference on Intelligent Robots and Systems, pages 6187-6193, Taipei, Taiwan, October 2010. 
A. Harnoy, B. Friedland, and S. Cohn. Modeling and measuring friction effects. IEEE Control Systems Magazine, 28(6), December 2008.

J. M. Hollerbach. A survey of kinematic calibration, pages 207-242. MIT Press, Cambridge, MA, USA, 1989.

A. Isaksson, A. C. Bittencourt, K. Forsman, and D. Peretzki. Method for controlling an industrial process, October 2010. URL http://patentscope. wipo.int/search/en/WO2012048734.

R. Isermann. Fault-Diagnosis Systems - An Introduction from Fault Detection to Fault Tolerance. Springer, 1st edition, 2006.

ISO 8373. Manipulating industrial robots - Vocabulary. ISO, Geneva, Switzerland, 1994.

B. Jacobson. The Stribeck memorial lecture. Tribology International, 36(11):781789, 2003.

T. M. Jahns and W. L. Soong. Pulsating torque minimization techniques for permanent magnet AC motor drives - a review. IEEE Transactions on Industrial Electronics, 43(2):321-330, 1996.

M. Jones and D. Henderson. Maximum likelihood kernel density estimation: On the potential of convolution sieves. Computational Statistics \& Data Analysis, 53(10):3726-3733, 2009.

M. Jonsson, A. Stolt, A. Robertsson, S. von Gegerfelt, and K. Nilsson. On force control for assembly and deburring of castings. Production Engineering, 7(4): 351-360, 2013.

F. I. Khan and S. A. Abbasi. Major accidents in process industries and an analysis of causes and consequences. Journal of Loss Prevention in the Process Industries, 12(5):361-378, 1999.

H. M. Kim, S. H. Park, and S. I. Han. Precise friction control for the nonlinear friction system using the friction state observer and sliding mode control with recurrent fuzzy neural networks. Mechatronics, 19(6):805-815, 2009.

S. Kotsiantis. Supervised machine learning: A review of classification techniques. Informatica, 31:249-268, 2007.

A. R. Lansdown, A. L. Price, and J. Larsen-Basse. Materials to resist wear - a guide to their selection and use. Journal of Tribology, 109(2):379-380, 1987.

N. Liu and K. Zhou. Optimal robust fault detection for linear discrete time systems. Journal of Control Science and Engineering, 7:1-16, January 2008.

L. Ljung. System Identification: Theory for the User. Prentice Hall PTR, 2nd edition, December 1999.

J. Machalíková, M. Sejkorová, J. Chýlková, and E. Schmidová. Application of tribodiagnostics in the maintenance of vehicles. In Proceedings of the 5th Inter- 
national Conference on Theoretical and Practical Issues in Transport, February 2010.

L. Marton and F. van der Linden. Temperature dependent friction estimation: Application to lubricant health monitoring. Mechatronics, 22(8):1078-1084, 2012.

R. Mattone and A. D. Luca. Relaxed fault detection and isolation: An application to a nonlinear case study. Automatica, 42(1):109 - 116, 2009.

M. McIntyre, W. Dixon, D. Dawson, and I. Walker. Fault identification for robot manipulators. IEEE Transactions on Robotics, 21(5):1028-1034, October 2005.

S. Moberg. Modeling and Control of Flexible Manipulators. Linköping studies in science and technology. Dissertations, no. 1349, Linköping Studies in Science and Technology, SE-581 83 Linköping, Sweden, December 2010.

Y.-R. Mohamed and E. El-Saadany. A current control scheme with an adaptive internal model for torque ripple minimization and robust current regulation in PMSM drive systems. IEEE Transactions on Energy Conversion, 23(1):92 -100 , March 2008.

M. Namvar and F. Aghili. Failure detection and isolation in robotic manipulators using joint torque sensors. Robotica, 2009.

H. Olsson, K. J. Åström, C. C. de Wit, M. Gafvert, and P. Lischinsky. Friction models and friction compensation. European Journal of Control, 4(3):176-195, 1998.

E. Parzen. On estimation of a probability density function and mode. The Annals of Mathematical Statistics, 33(3):1065-1076, 1962.

D. Peretzki, A. J. Isaksson, A. C. Bittencourt, and K. Forsman. Data mining of historic data for process identification. In Proceedings of the 2011 AIChE Annual Meeting, October 2011.

A. Proca, A. Keyhani, A. El-Antably, W. Lu, and M. Dai. Analytical model for permanent magnet motors with surface mounted magnets. IEEE Transactions on Energy Conversion, 18(3):386 - 391, September 2003.

B. K. N. Rao. Condition monitoring and the integrity of industrial systems. In A. Davies, editor, Part 1: Introduction to Condition Monitoring, Handbook of Condition Monitoring - Techniques and Methodology, chapter 1, pages 3-34. Chapman \& Hall, London, UK, 1998.

M. D. Reid and R. C. Williamson. Information, divergence and risk for binary experiments. Journal of Machine Learning Research, 12:731-817, 2011.

R. Reiter. A theory of diagnosis from first principles. Artificial Intelligence, 32 (1):57 - 95, 1987. ISSN 0004-3702.

B. Roylance. Ferrography - then and now. Tribology International, 38(10):857 862, 2005. 
P. Rzeszucinski, J. Sinha, R. Edwards, A. Starr, and B. Allen. Amplitude of probability density function (APDF) of vibration response as a robust tool for gearbox diagnosis. Strain, 48(6):510-516, 2012.

A. Samuelsson, A. C. Bittencourt, K. Saarinen, S. S. Tavallaey, M. Norrlöf, H. Andersson, and S. Gunnarsson. Simulation based evaluation of fault detection algorithms with applications to wear diagnosis in manipulators. In Proceedings of the 19th IFAC World Congress, Cape Town, South Africa, 2014.

S. Sander-Tavallaey and K. Saarinen. Backlash identification in transmission unit. In Proceedings of the 2009 IEEE Control Applications \& Intelligent Control, pages 1325 -1331, July 2009. doi: 10.1109/CCA.2009.5281173.

S. Sander-Tavallaey, K. Saarinen, H. Andersson, and A. C. Bittencourt. Condition monitoring of an industrial robot, October 2012. URL http:// patentscope.wipo.int/search/en/WO2013050314.

L. Sciavicco and B. Siciliano. Modelling and control of robot manipulators. Advanced textbooks in control and signal processing. Springer, 2000.

T. Sebastian. Temperature effects on torque production and efficiency of pm motors using ndfeb magnets. IEEE Transactions on Industry Applications, 31(2): 353-357, October 1995.

C. J. Seeton. Viscosity-temperature correlation for liquids. Tribology Letters, 22 (1):67-78, March 2006.

W. Seifert and V. Westcott. A method for the study of wear particles in lubricating oil. Wear, 21(1):27 - 42, 1972.

SKF. Interactive engineering catalogue, August 2011. URL http://www.skf . $\mathrm{com} /$ portal/skf/home/products? newlink=first\&lang=en.

M. Spong, S. Hutchinson, and M. Vidyasagar. Robot modeling and control. John Wiley \& Sons, 2006.

M. W. Spong. Modeling and control of elastic joint robots. Journal of Dynamic Systems, Measurement, and Control, 109(4):310-318, 1987.

R. Stribeck. Die wesentlichen eigenschaften der gleit- und rollenlager - The key qualities of sliding and roller bearings. Zeitschrift Des Vereins Deutscher Ingenieure, 46(38-39):1342-1437, 1902.

W. Susanto, R. Babuska, F. Liefhebber, and T. van der Weiden. Adaptive friction compensation: application to a robotic manipulator. In Proceedings of 17th IFAC World Congress, Seoul, South Korea, December 2008.

J. Swevers, C. Ganseman, D. Tukel, J. de Schutter, and H. Van Brussel. Optimal robot excitation and identification. IEEE Transactions on Robotics and $\mathrm{Au}$ tomation, 13(5):730 - 740, October 1997.

J. I. Taylor. The Vibration Analysis Handbook. Vibration Consultants, February 1994. 
D. Tencer. Global robot population grew $12 \%$ in 2013 , and that's just the beginning, June 2013. URL http://www.huffingtonpost.ca/2014/06/12/ global-robot-population_n_5489454.html.

M. Tenerz. Parameter Estimation in a Permanent Magnet Synchronous Motor. Msc. thesis LiTH-ISY-EX-11/4495-SE, Department of Electrical Engineering, Linköping University, 2011.

G. Thompson. Improving maintainability and reliability through design. Wiley, 1st edition, January 1999.

A. Tustin. The effects of backlash and of speed-dependent friction on the stability of closed-cycle control systems. Journal of the Institution of Electrical Engineers - Part IIA: Automatic Regulators and Servo Mechanisms, 94(1):143151, May 1947.

H. L. Van Trees. Detection, Estimation and Modulation Theory, Part I. Wiley, New York, 1st edition, 2001.

A. T. Vemuri and M. M. Polycarpou. A methodology for fault diagnosis in robotic systems using neural networks. Robotica, 22(04):419-438, 2004.

R. Waiboer, R. Aarts, and B. Jonker. Velocity dependence of joint friction in robotic manipulators with gear transmissions. In Proceedings of the 2005 ECCOMAS Thematic Conference Multibody Dynamics, pages 1-19, Madrid, Spain, 2005.

E. Wernholt. Multivariable Frequency-Domain Identification of Industrial Robots. Linköping studies in science and technology. Dissertations. no. 1138, Linköping Studies in Science and Technology, SE-581 83 Linköping, Sweden, November 2007.

E. Wernholt and S. Moberg. Nonlinear gray-box identification using local models applied to industrial robots. Automatica, 47(4):650 - 660, 2011.

L. Westerlund. The extended arm of man - a history of the industrial robot. Technical report, Informationsförlaget, Stockholm, Sweden, 2000.

M. Woydt and R. Wäsche. The history of the Stribeck curve and ball bearing steels: The role of Adolf Martens. Wear, 268(11-12):1542-1546, 2010.

J. Wu, J. Wang, and Z. You. An overview of dynamic parameter identification of robots. Robotics and Computer-Integrated Manufacturing, 26(5):414 - 419, 2010.

D.-Y. Yeung and C. Chow. Parzen-window network intrusion detectors. In Proceedings of the 16th International Conference on Pattern Recognition, volume 4, pages 385-388, Quebec, Canada, 2002. IEEE.

S. Yin, S. X. Ding, A. Haghani, H. Hao, and P. Zhang. A comparison study of basic data-driven fault diagnosis and process monitoring methods on the bench- 
mark Tennessee Eastman process. Journal of Process Control, 22(9):1567-1581, 2012.

O. Zeitouni, J. Ziv, and N. Merhav. When is the generalized likelihood ratio test optimal? IEEE Transactions on Information Theory, 38(5):1597-1602, 1992. 


\section{Part II}

\section{Publications}





\section{Paper A}

\section{Friction in a Robot Joint - Modeling and Identification of Load and Temperature Effects}

Authors:

André Carvalho Bittencourt and Svante Gunnarsson.

Edited version of the paper

A. C. Bittencourt and S. Gunnarsson. Static friction in a robot joint modeling and identification of load and temperature effects. Journal of Dynamic Systems, Measurement, and Control, 134(5), July 2012.

Parts of this paper were previously published in:

A. C. Bittencourt, E. Wernholt, S. Sander-Tavallaey, and T. Brogårdh. An extended friction model to capture load and temperature effects in robot joints. In Proceedings of the 2010 IEEE/RSJ International Conference on Intelligent Robots and Systems, pages 6161-6167, Taipei, Taiwan, October 2010.

Preliminary version:

Technical Report LiTH-ISY-R-3038, Dept. of Electrical Engineering, Linköping University, SE-581 83 Linköping, Sweden. 



\title{
Friction in a Robot Joint - Modeling and Identification of Load and Temperature Effects
}

\author{
André Carvalho Bittencourt and Svante Gunnarsson \\ Dept. of Electrical Engineering, \\ Linköping University, \\ SE-581 83 Linköping, Sweden \\ \{andrecb, svante\} @isy.liu.se
}

\begin{abstract}
Friction is the result of complex interactions between contacting surfaces in down to a nanoscale perspective. Depending on the application, the different models available are more or less suitable. Friction models in robotics are typically considered to be dependent only on joint speed. However, it is known that friction can be affected by other factors.

In this paper, the typical friction phenomena and models used in robotics are reviewed. It is shown how such models can be represented as a sum of functions of relevant states which are linear and nonlinear in the parameters. The identification method described in Golub and Pereyra (1973) is suggested for parameter identification when all states are available. The discussion follows with a detailed experimental study of friction in a robot joint under changes of joint angle, load torque and temperature. Justified by their significance, load torque and temperature are included in an extended friction model. The proposed model is validated in a wide operating range, considerably improving the prediction performance compared to a standard model.
\end{abstract}

\section{Introduction}

Friction exists in all mechanisms to some extent. It can be defined as the tangential reaction force between two surfaces in contact. It is a nonlinear phenomenon which is physically dependent on contact geometry, topology, properties of the materials, relative velocity, lubricant, etc. (Al-Bender and Swevers, 2008). Friction has been constantly investigated by researchers due to its importance in several fields, see e.g. Dowson (1998). In this paper, friction has been studied in industrial robot joints based on experiments. 
In robotics, one reason for the interest in friction is the need to model friction for control purposes, see e.g. Kim et al. (2009); Guo et al. (2008); Olsson et al. (1998); Bona and Indri (2005); Susanto et al. (2008). A precise friction model can considerably improve the overall performance of a manipulator with respect to accuracy and control stability. Since friction can relate to the wear processes of mechanical systems (Blau, 2009), including robot joints (Bittencourt et al., 2011), there is also interest in friction modeling for robot condition monitoring and fault detection, see e.g. Freyermuth (1991); Vemuri and Polycarpou (2004); McIntyre et al. (2005); Mattone and Luca (2009); Brambilla et al. (2008); Caccavale et al. (2009); Namvar and Aghili (2010); Bittencourt et al. (2011).

A friction model consistent with real experiments is necessary for successful simulation, design and evaluation. Due to the complexity of friction, it is often difficult to obtain models that can describe all the empirical observations. See Al-Bender and Swevers (2008) for a comprehensive discussion on friction physics and first principle friction modeling. In a robot joint, the complex interactions of components such as gears, bearings and shafts which are rotating/sliding at different velocities, makes physical modeling difficult. An example of an approach to model friction of complex transmissions can be found in Waiboer (2007), where the author designs joint friction models based on physical models of elementary joint components such as helical gear pairs and pre-stressed roller bearings.

Empirically motivated friction models have been successfully used in many applications, including robotics, see e.g. Armstrong-Hélouvry (1991); Olsson et al. (1998); Åström and Canudas-de Wit (2008); Harnoy et al. (2008). This category of models was developed through time according to empirical observations of the phenomenon. Considering a set of states $x$, and parameters $\theta$, these model structures $\mathcal{M}$, can be described as,

$$
f(\boldsymbol{x}, \boldsymbol{\theta})=\sum_{j=1}^{M} \phi_{j}(\boldsymbol{x}, \boldsymbol{\theta}),
$$

where $f$ is the generalized friction and $\phi_{j}$ are general nonlinear functions or regressors. This static nonlinear relation can be extended further to include dynamics by considering additional differential equations

$$
\dot{x}=\sum_{j=1}^{M_{d}} \phi_{j}^{d}(\boldsymbol{x}, \boldsymbol{\theta}) .
$$

The choice $x=[z, \dot{q}, q]^{T}$, where $q$ is a generalized coordinate and $z$ is an internal state vector related to the dynamic behavior of friction, with dynamics described by a first-order differential equation, gives the set of Generalized Empirical Friction Model structures (GEFM), see Al-Bender and Swevers (2008).

Among the GEFM model structures, the LuGre model (Olsson et al., 1998) is a common choice in the robotics community. For a revolute joint, the LuGre model 
structure $\mathcal{M}^{L}$, can be described as

$$
\begin{aligned}
\tau^{f} & =\sigma_{0} z+\sigma_{1} \dot{z}+h(\dot{\varphi}) \\
\dot{z} & =\dot{\varphi}-\sigma_{0} \frac{|\dot{\varphi}|}{g(\dot{\varphi})} z,
\end{aligned}
$$

where $\tau^{f}$ is the friction torque, $\varphi$ is the joint motor angle. The state $z$ is related to the dynamic behavior of asperities in the interacting surfaces and can be interpreted as their average deflection, with stiffness $\sigma_{0}$ and damping $\sigma_{1}$.

The function $h(\dot{\varphi})$ represents the velocity strengthening (viscous) friction and is dependent on the stress versus strain rate relationship of the lubricant. For Newtonian fluids, the shear stress follows a linear dependency to the shear rate, $\tau=\mu \frac{\partial u}{\partial y}$, where $\tau$ is the shear stress, $\frac{\partial u}{\partial y}$ is the local shear rate and $\mu$ is the viscosity. It is typical to consider a Newtonian behavior, yielding the relationship

$$
h(\dot{\varphi})=f_{v} \dot{\varphi}
$$

for the viscous behavior of friction.

The function $g(\dot{\varphi})$ captures the velocity weakening of friction. Motivated by the observations mainly attributed to Stribeck (Jacobson, 2003; Woydt and Wäsche, 2010; Bo and Pavelescu, 1982), $g(\dot{\varphi})$ is commonly modeled as

$$
g(\dot{\varphi})=f_{c}+f_{s} e^{-\left|\frac{\dot{\varphi}}{\varphi_{s}}\right|^{\alpha}},
$$

where $f_{c}$ is the Coulomb friction, $f_{s}$ is called the standstill friction parameter ${ }^{\mathrm{i}}, \dot{\varphi}_{s}$ is the Stribeck velocity and $\alpha$ is the exponent of the Stribeck nonlinearity. The model structure $\mathcal{M}^{L}$ with the $h$ - and $g$ functions given by (1) and (2) is a GEFM with

$$
\boldsymbol{x}=\left[\begin{array}{ll}
z, & \dot{\varphi}
\end{array}\right]^{T}, \quad \boldsymbol{\theta}=\left[\begin{array}{lllllll}
\sigma_{0}, & \sigma_{1}, & f_{c}, & f_{s}, & f_{v}, & \dot{\varphi}_{s}, & \alpha
\end{array}\right]^{T} .
$$

According to Åström and Canudas-de Wit (2008), the LuGre model can successfully describe many of the friction characteristics.

Since $z$ is not measurable, a difficulty with $\mathcal{M}^{L}$ is the estimation of the dynamic parameters $\left[\sigma_{0}, \sigma_{1}\right]$. In Olsson et al. (1998), these parameters are estimated in a robot joint by means of open loop experiments. Open-loop experiments are not always possible, and it is common to accept only a static description of $\mathcal{M}^{L}$. In steady-state, $\mathcal{M}^{L}$ is equivalent to the steady-state model $\mathcal{M}^{S}$ :

$$
\tau^{f}(\dot{\varphi})=g(\dot{\varphi}) \operatorname{sign}(\dot{\varphi})+h(\dot{\varphi})
$$

which is fully described by the $g$ - and $h$ functions. In fact, $\mathcal{M}^{L}$ adds dynamics to $\mathcal{M}^{S}$. The typical choice for $g-$ and $h$, as defined in (2) and (1), yields the static

\footnotetext{
${ }^{\mathrm{i}}$ The parameter $f_{s}$ is commonly denoted static friction, describing the friction value close to zero speed. An alternative nomenclature was adopted to make a distinction between the dynamic (differential) and static (algebraic) description of friction.
} 
model structure $\mathcal{M}^{0}$ :

$$
\tau^{f}(\dot{\varphi})=\left[f_{c}+f_{s} e^{-\left|\frac{\dot{\varphi}}{\varphi_{s}}\right|^{\alpha}}\right] \operatorname{sign}(\dot{\varphi})+f_{v} \dot{\varphi} .
$$

This model structure is commonly used and was described in Bo and Pavelescu (1982). For the fixed $\alpha=1, \mathcal{M}^{0}$ simplifies to the Tustin model, first introduced in Tustin (1947). The model $\mathcal{M}^{0}$ requires a total of four parameters to describe the velocity weakening regime, $g(\dot{\varphi})$, and one parameter to capture viscous friction, $h(\dot{\varphi})$. See Section 3 for a description of friction velocity regimes and an interpretation of the parameters.

From empirical observations, it is known that friction can be affected by several factors, e.g.:

- temperature,

- force/torque levels,

- position,
- velocity,

- acceleration,

- lubricant properties.

A shortcoming with the LuGre model structure, as with any GEFM, is the dependence only of the states $x=[z, \dot{q}, q]$. In more demanding applications, the effects of the remaining variables can not be neglected. In Waiboer et al. (2005), the author observes a strong temperature dependence, while in Olsson et al. (1998), joint load torque and temperature are considered as disturbances and estimated in an adaptive framework. In Gogoussis and Donath (1988); Dohring et al. (1993), the effects of load are modeled as a linear effect on $f_{c}$, in a model structure similar to $\mathcal{M}^{0}$. In the recent contribution of Hamon et al. (2010) the effects of load are revisited to include also a linear dependency on $f_{s}$. However, more work is needed in order to understand the influence of the different factors to the friction properties. A more comprehensive friction model is needed to improve tasks related to design, control, simulation and evaluation in industrial robotics.

The objective of this paper is to analyze and model the effects of speed, joint angle, load torques and temperature to friction in a robot joint. Only the steadystate behavior of friction is studied and possible dynamic effects of friction are not considered. The phenomena are studied in joint 2 of an ABB IRB 6620 industrial robot, see Figure 1a. Two load torque components are examined, the torque aligned to the joint degree of freedom (DOF) and the resulting torque perpendicular to the joint's DOF. These torques are in the paper named manipulation load torque $\tau^{\ell}$ and perpendicular load torque $\tau^{p}$, see Figure $1 \mathrm{~b}$.

By means of experiments, these variables are analyzed and an empirically motivated model is proposed. The task of modeling is to find a suitable static model structure according to:

$$
\begin{aligned}
\tau^{f}\left(\boldsymbol{x}^{*}, \boldsymbol{\theta}\right) & =\sum_{j=1}^{M} \phi_{j}\left(\boldsymbol{x}^{*}, \boldsymbol{\theta}\right) \\
\boldsymbol{x}^{*} & =\left[\dot{\varphi}, \varphi^{a}, \tau^{p}, \tau^{\ell}, \xi\right],
\end{aligned}
$$




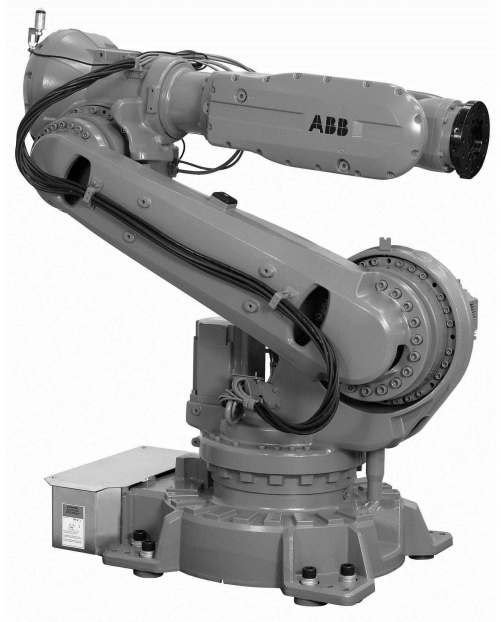

(a) $A B B$ IRB 6620 robot with $150 \mathrm{~kg}$ payload and $2.2 \mathrm{~m}$ reach.

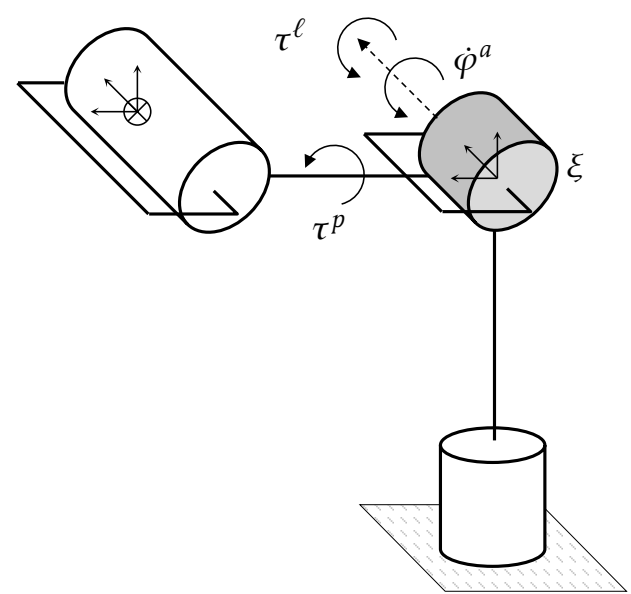

(b) Schematics of the three first joints including the torque definitions for joint 2.

Figure 1: The experiments were performed for joint 2 of an $A B B$ robot IRB 6620. The variable $\varphi^{a}$ is the joint angle, $\xi$ is the joint lubricant temperature, $\tau^{\ell}$ is the manipulation load torque and $\tau^{p}$ is the perpendicular load torque.

where $\xi$ is the joint (more precisely, lubricant $\mathrm{t}^{\mathrm{i}}$ ) temperature and $\varphi^{a}$ is the joint angle at the arm side, i.e. after the gearbox.

Ideally, the chosen model should be coherent with the empirical observations and, simultaneously, with the lowest dimension of $\theta$, the parameter vector, and with the lowest number of describing functions, i.e. small $M$. For practical purposes, the choice of $\phi_{j}(\cdot)$ should also be suitable for a simple identification procedure.

The work presented here is based on Bittencourt et al. (2010), where a friction model was proposed to describe the effects of load and temperature in a robot joint. More detailed analysis of the modeling assumptions are presented, together with a more general framework for identification of friction models. The paper is organized as follows. Section 2 presents an identification method for general friction models when all states are available. Section 3 reviews basic friction phenomena and models for robot joints and presents an experiment to retrieve friction data from a robot joint. In Section 4, a velocity-dependent friction model is extended based on empirical observations to propose and validate a model structure for $\mathcal{M}^{*}$. Conclusions and future work are discussed in Section 5.

\footnotetext{
${ }^{\mathrm{i}}$ In the studies, the robot gearbox was lubricated with oil, not grease, which gave an opportunity to obtain well defined temperature readings by having a temperature sensor in the circulating oil.
} 


\section{Identification of Friction Models}

For a given value of the friction states $x_{k}$, the associated friction value $\tau_{k}^{f}$ can be predicted by a static model description $\mathcal{M}$. Denoting $\widehat{\tau}^{f}(k \mid \theta)$ the predicted value with model parameters $\theta$, the error $\epsilon(k, \theta) \triangleq \tau_{k}^{f}-\widehat{\tau}^{f}(k \mid \theta)$ measures deviations between the model and observations. A common criterion for the choice of $\theta$ is the value giving the least sum of squared errors. For $N$ observations, the least squares estimate is given by

$$
\widehat{\boldsymbol{\theta}}_{N}=\arg \min _{\theta} V_{N}(\boldsymbol{\theta})=\arg \min _{\theta} \frac{1}{N} \sum_{k=1}^{N} \frac{1}{2} \epsilon^{2}(k, \theta) .
$$

The minimum of (3) occurs where the gradient of the cost function $V_{N}(\theta)$ is zero, i.e.

$$
\frac{\partial}{\partial \theta} V_{N}(\boldsymbol{\theta})=-\frac{1}{N} \sum_{k=1}^{N} \psi(k \theta) \epsilon(k, \theta)=\mathbf{0},
$$

where $\psi(k, \theta) \triangleq-\frac{\partial}{\partial \theta} \varepsilon(k, \theta)$, and a nonlinear system of equations needs to be solved.

In many cases, friction models will contain subsets of parameters that appear linearly in the functions $\phi_{j}(\cdot)$. Denoting this subset of parameters $\eta$, an alternative description of a friction model is

$$
\tau^{f}(\boldsymbol{x}, \boldsymbol{\theta})=\sum_{j=1}^{N_{\eta}} \phi_{j}(\boldsymbol{x}, \boldsymbol{\rho}) \eta_{j}=\phi(\boldsymbol{x}, \boldsymbol{\rho})^{T} \eta
$$

where the parameter vector $\theta^{T}=\left[\begin{array}{ll}\eta^{T}, & \rho^{T}\end{array}\right]$ has dimension $\left(N_{\eta}+N_{\rho}\right)$ and is divided according to the manner they appear in the model, respectively linearly or nonlinearly. For the model in (4), the gradient $\psi(k, \theta)$ takes the form

$$
\psi(k, \theta)=\left[\phi\left(x_{k}, \rho\right)^{T}, \quad\left(\frac{\partial}{\partial \rho} \phi\left(x_{k}, \rho\right)^{T} \eta\right)^{T}\right]^{T},
$$

where it is easy to realize the separable nature of the model. As presented in Golub and Pereyra (1973), the separable structure of the model can be explored for identification. Defining the matrix $[\Phi(\rho)]_{k, j}=\phi_{j}\left(\boldsymbol{x}_{k}, \boldsymbol{\rho}\right) \eta_{j}$, for any given $\boldsymbol{\rho}$, the solution for $\eta$ is given by the ordinary least squares estimate

$$
\widehat{\eta}=\Phi^{\dagger}(\rho) \tau^{f}, \quad \Phi^{\dagger}(\rho) \triangleq\left(\Phi^{T}(\rho) \Phi(\rho)\right)^{-1} \Phi^{T}(\rho), \quad \tau^{f} \triangleq\left[\tau_{1}^{f}, \quad \cdots, \quad \tau_{N}^{f}\right]^{T}
$$

where $\Phi^{\dagger}(\rho)$ is the Moore-Penrose pseudoinverse of $\Phi(\rho)$ and $\tau^{f}$ is the vector of observations. Substituting this back in (3), the problem can be rewritten as a function only of $\rho$, thus reducing the amount of parameters in the nonlinear 
minimization. The resulting nonlinear problem is given as

$$
\widehat{\boldsymbol{\rho}}=\arg \min _{\rho}\left\|\boldsymbol{\tau}^{f}-\Phi(\rho) \widehat{\eta}\right\|^{2}=\arg \min _{\rho}\left\|P_{\Phi(\boldsymbol{\rho})}^{\perp} \boldsymbol{\tau}^{f}\right\|^{2}, \quad P_{\Phi(\boldsymbol{\rho})}^{\perp} \triangleq I-\Phi(\rho) \Phi^{\dagger}(\boldsymbol{\rho})
$$

where $P_{\Phi(\rho)}^{\perp}$ is the projector on the orthogonal complement of the column space of $\Phi(\rho)$. The idea for an identification procedure is thus to first find $\widehat{\rho}$ from $(7)$, and then plug it back in (6) to find $\widehat{\eta}$. This illustrates the algorithm proposed in Golub and Pereyra (1973), where it is also shown that the resulting point $\widehat{\theta}^{T}=$ $\left[\widehat{\eta}^{T}, \widehat{\rho}^{T}\right]$ is a minimum of (3).

The nonlinear minimization in (7) can be solved using gradient based methods. In Golub and Pereyra (1973), it is shown that the gradient of $P_{\Phi(\rho)}^{\perp} \tau^{f}$ requires only computation of derivatives of $\Phi(\rho)$, as in (5), see Golub and Pereyra (1973) for a detailed treatment. In this work, (7) is solved using a trust-region reflective algorithm available in the Matlab's Optmization Toolbox with initial estimates given by a coarse grid search. The resulting estimate $\widehat{\rho}$ is plugged in (6) to find $\widehat{\eta}$.

\subsection{Covariance estimate}

As presented in Ljung (1999), in case there is a parameter value $\theta^{0}$ such that $\epsilon\left(k, \theta^{0}\right)$ is a sequence of independent zero mean random variables with variance $\gamma^{0}$, then as the number of data points $N$ tends to infinity, the least squares estimate $\widehat{\boldsymbol{\theta}}_{N}$ will converge in distribution as

$$
\sqrt{N}\left(\widehat{\boldsymbol{\theta}}_{N}-\boldsymbol{\theta}^{0}\right) \in A s \mathcal{N}\left(0, P_{\boldsymbol{\theta}}\right)
$$

where $P_{\boldsymbol{\theta}}$ is given by

$$
P_{\boldsymbol{\theta}}=\gamma^{0}\left[\lim _{N \rightarrow \infty} \frac{1}{N} \sum_{k=1}^{N} \mathbb{E} \boldsymbol{\psi}\left(k, \boldsymbol{\theta}^{0}\right) \boldsymbol{\psi}\left(k, \boldsymbol{\theta}^{0}\right)^{T}\right]
$$

and the asymptotic covariance for $\widehat{\boldsymbol{\theta}}_{N}$ is thus $\Sigma_{\boldsymbol{\theta}}=\frac{1}{N} P_{\boldsymbol{\theta}}$. The finite sample estimate of $\Sigma_{\boldsymbol{\theta}}$ is given by

$$
\begin{aligned}
\widehat{\Sigma}_{\widehat{\boldsymbol{\theta}}_{N}} & =\widehat{\gamma}_{N}\left[\sum_{k=1}^{N} \psi\left(k, \widehat{\boldsymbol{\theta}}_{N}\right) \psi\left(k, \widehat{\boldsymbol{\theta}}_{N}\right)^{T}\right]^{-1} \\
\widehat{\gamma}_{N} & =\frac{1}{N} \sum_{k=1}^{N} \epsilon^{2}\left(k, \widehat{\boldsymbol{\theta}}_{N}\right) .
\end{aligned}
$$

The quantity in (10) is used throughout this work as a covariance estimate for $\widehat{\boldsymbol{\theta}}_{N}$ and provides a measure of accuracy for the resulting estimates. 


\section{Basics of Friction Phenomena in a Robot Joint}

Friction is typically presented in a friction curve, a plot of friction levels against speed achieved in stationary conditions. The friction curve is related to the Stribeck curve (Woydt and Wäsche, 2010) under the simplification that viscosity and load are constant. An example of a friction curve for a robot joint can be seen in Figure 3.

From a phenomenological perspective, a friction curve can be divided into three regimes, according to the lubrication characteristics: boundary (BL), mixed (ML) and elasto-hydrodynamic lubrication (EHL). The phenomena present in very low velocities (BL) is mostly related to interactions between the asperities of the surfaces in contact. With the increase of velocity, there is a consequent increase of the lubrication film between the surfaces and a decrease of friction (ML) until it reaches a full lubrication profile (EHL) with a separation of the surfaces by the lubricant. In EHL, friction is proportional to the force needed to shear the lubricant layer, thus dependent on the lubricant properties, in particular its viscosity. Recalling the steady-state friction model $\mathcal{M}^{S}$, the BL and ML regimes are described by the velocity weakening function $g$ - and the EHL regime is described by $h$.

In the next subsection, an experimental procedure is suggested to retrieve the friction curve of a robot joint based on constant-speed experiments. Using the identification method described in Section 2, different models to describe the observed speed dependency are found and some model simplifications are motivated.

\subsection{A procedure to estimate friction at a fixed speed level}

A manipulator is a multivariable, nonlinear system that can be described in a general manner through the rigid multi-body dynamic model

$$
M(\varphi) \ddot{\varphi}+C(\varphi, \dot{\varphi}) \dot{\varphi}+\tau^{g}(\varphi)+\tau^{f}(\dot{\varphi})=\tau,
$$

where $M(\varphi)$ is the inertia matrix, $C(\varphi, \dot{\varphi})$ relates to speed dependent terms (Coriolis and centrifugal), $\tau^{g}(\varphi)$ are the gravity-induced torques and $\boldsymbol{\tau}^{f}$ contains the friction torques. The system is controlled by the input torque, $\tau$, applied by the joint motor (in the experiments the torque reference from the servo was measured $\left.^{\mathrm{i}}\right)$.

For single joint movements (so that centrifugal forces are zero at that joint) and under constant speed (so that inertial torques are zero), the applied torque at the joint under actuation simplifies to

$$
\tau^{g}(\varphi)+\tau^{f}(\dot{\varphi})=\tau
$$

and drives only friction and gravity-induced torques. The required torques to drive a joint in forward, $\tau^{+}$, and reverse, $\tau^{-}$, directions at the constant speed

\footnotetext{
${ }^{\mathrm{i}}$ It is known that using the torque reference from the servo as a measure of the joint torque might not always hold because of the temperature dependence of the torque constant of the motors. The deviations are however considered to be small and are neglected during the experiments.
} 


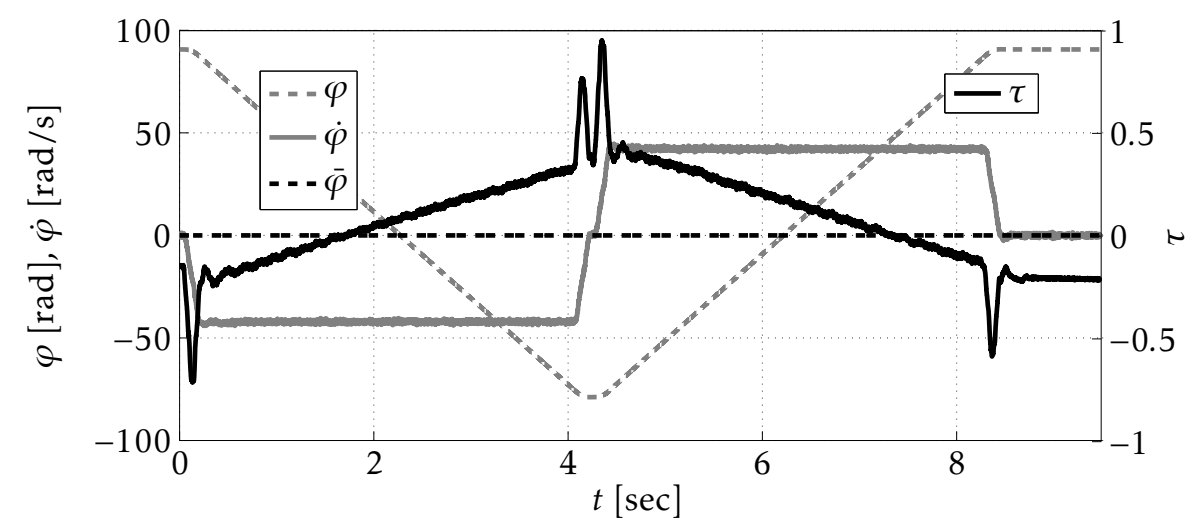

Figure 2: Excitation signals used for the friction estimation at $\overline{\dot{\varphi}}=42 \mathrm{rad} / \mathrm{s}$ and $\bar{\varphi}=0 \mathrm{rad}$.

level $\overline{\dot{\varphi}}$, and at a joint angle value $\bar{\varphi}$ (so that $\tau^{g}(\bar{\varphi})$ is equal in both directions), are

$$
\tau^{f}(\overline{\dot{\varphi}})+\tau^{g}(\bar{\varphi})=\tau^{+} \quad \tau^{f}(-\overline{\dot{\varphi}})+\tau^{g}(\bar{\varphi})=\tau^{-} .
$$

In the case an estimate of $\tau^{g}(\bar{\varphi})$ is available, it is possible to isolate the friction component in each direction using Equation (14). If such estimate is not possible (e.g. not all masses are completely known), $\tau^{f}$ can still be retrieved in the case that $\tau^{f}$ is independent of the rotation direction. Subtracting the equations yields

$$
\tau^{f}(\overline{\dot{\varphi}})-\tau^{f}(-\overline{\dot{\varphi}})=\tau^{+}-\tau^{-}
$$

and if $\tau^{f}(-\overline{\dot{\varphi}})=-\tau^{f}(\overline{\dot{\varphi}})$, the resulting direction independent friction is:

$$
\tau^{f}(\overline{\dot{\varphi}})=\frac{\tau^{+}-\tau^{-}}{2} \text {. }
$$

In the experiments, each joint is moved separately with the desired speed in both directions around a given joint angle $\bar{\varphi}$. Figure 2 shows the measured joint angle-, speed- and torque ${ }^{i}$ signals for $\overline{\dot{\varphi}}=42 \mathrm{rad} / \mathrm{s}$ around $\bar{\varphi}=0 \mathrm{rad}$. The constant speed data is segmented around $\bar{\varphi}$ and the friction levels can be achieved using Equation (14) or (15).

The procedure can be repeated for several different speeds and a friction curve can be drawn, as displayed by the circles in Figure 3. As shown in Figure 3, there is only a small direction dependency of friction for the investigated joint. Therefore, in this paper, friction levels are achieved using Equation (15), which is not influenced by deviations in the gravity model of the robot.

\footnotetext{
${ }^{\mathrm{i}}$ Throughout the paper all torques are normalized to the maximum manipulation torque at low speed and are therefore presented as dimensionless quantities.
} 
Table 1: Identified $\mathcal{M}^{0}$ parameters for the data shown in Figure 3.

\begin{tabular}{ccccc}
$f_{c}\left[10^{-2}\right]$ & $f_{s}\left[10^{-2}\right]$ & $f_{v}\left[10^{-4}\right]$ & $\dot{\varphi}_{s}$ & $\alpha$ \\
$3.4 \pm 0.176$ & $4.6 \pm 0.48$ & $3.68 \pm 0.12$ & $10.68 \pm 1.08$ & $1.93 \pm 0.60$ \\
\hline
\end{tabular}

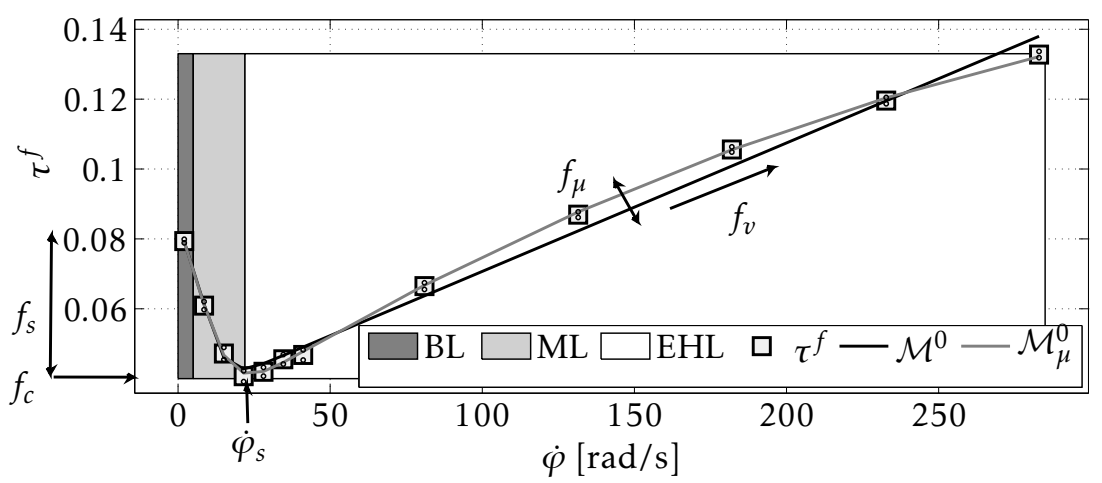

Figure 3: Friction curve with lubrication regimes and model-based predictions. Squares indicate friction levels achieved using Equation (15), the (almost indistinguishable) superimposed circles are friction levels achieved using Equation (14).

\subsection{Modeling of velocity dependencies}

Model structure $\mathcal{M}^{0}$ is a common choice to describe the friction dependency with speed. For a direction independent friction, it suffices to describe friction for positive velocities, which is a convention adopted from here and on. For positive velocities, $\mathcal{M}^{0}$ can be written as in Equation (4) with

$$
\begin{aligned}
& x=\dot{\varphi}, \\
& \eta=\left[\begin{array}{lll}
f_{c}, & f_{s}, & f_{v}
\end{array}\right]^{T}, \quad \boldsymbol{\rho}=\left[\begin{array}{ll}
\dot{\varphi}_{s}, & \alpha
\end{array}\right]^{T} .
\end{aligned}
$$

The model parameters are identified using the direction independent data (circles) in Figure 3. The resulting identified parameters values are shown in Table 1 with one standard deviation. The dark solid line in Figure 3 is obtained by modelbased predictions of the resulting model, with sum of absolute errors smaller than $3.010^{-2}$.

A closer investigation of the friction curve in Figure 3 reveals that the behavior of friction at high speeds is slightly nonlinear with speed. This feature is related to the non-Newtonian behavior of the lubricant at high speeds, see e.g. Waiboer et al. (2005). In this case, the fluid presents a pseudoplastic behavior, with a decrease of the apparent viscosity with shear rate. The behavior motivates 


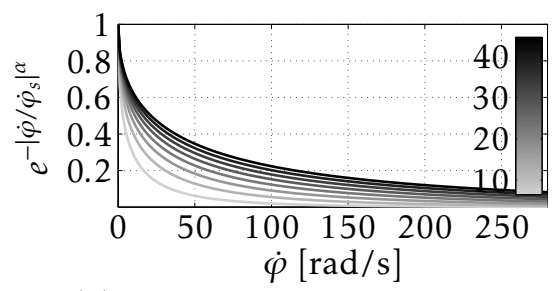

(a) $\alpha=0.5, \dot{\varphi}_{s} \in(1,50) \mathrm{rad} / \mathrm{s}$.

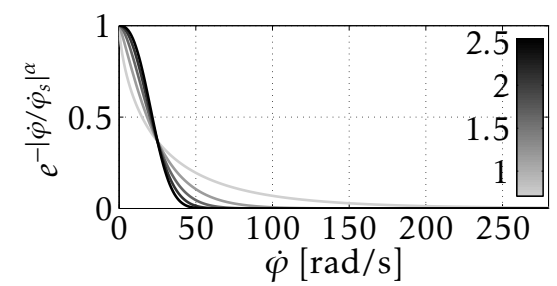

(c) $\dot{\varphi}_{s}=25 \mathrm{rad} / \mathrm{s}, \alpha \in(0.02,3.00)$.

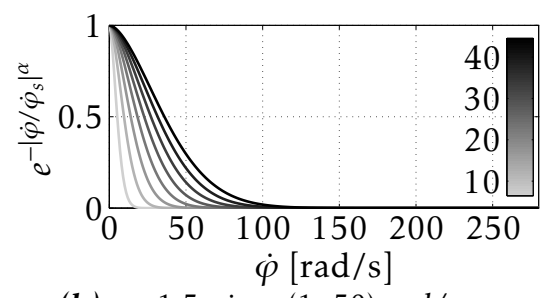

(b) $\alpha=1.5, \dot{\varphi}_{s} \in(1,50) \mathrm{rad} / \mathrm{s}$.

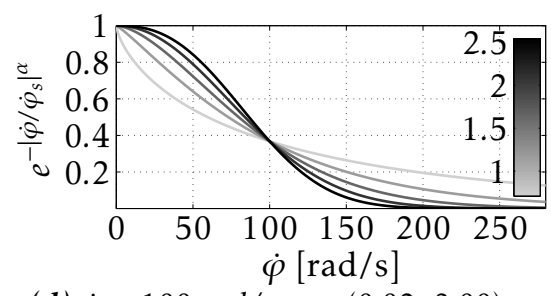

(d) $\dot{\varphi}_{s}=100 \mathrm{rad} / \mathrm{s}, \alpha \in(0.02,3.00)$.

Figure 4: Effects of changes of $\dot{\varphi}_{s}$ and $\alpha$ to the friction curve.

the suggestion of an alternative model structure

$$
\tau^{f}(\dot{\varphi})=f_{c}+f_{s} e^{-\left|\frac{\dot{\varphi}}{\varphi_{s}}\right|^{\alpha}}+f_{v} \dot{\varphi}+f_{\mu} \dot{\varphi}^{\beta},
$$

where $f_{\mu}$ and $\beta$ relate to the non-Newtonian part of the viscous friction behavior and capture the deviation from a Newtonian behavior. The parameters for this model are identified for the friction curve in Figure 3. The resulting predictions are shown by the gray solid line in Figure 3, with sum of absolute prediction error of $5.510^{-3}$.

Despite the non-Newtonian behavior of the lubricant, the increase in accuracy achieved with $\mathcal{M}_{\mu}^{0}$ is relatively small compared to $\mathcal{M}^{0}$. As it will be shown, other effects are considerably more significant. For simplicity of the resulting model, $\mathcal{M}^{0}$ is considered as a basis to describe the dependencies with speed and the nonNewtonian behavior is not considered further in this paper.

\section{Fixing $\alpha$}

The model $\mathcal{M}^{0}$ describes the velocity weakening regime, $g(\cdot)$, through the exponential term $e^{-\left|\frac{\dot{\varphi}}{\varphi_{s}}\right|^{\alpha}}$ and takes two nonlinear parameters $\alpha$ and $\dot{\varphi}_{s}$. To further simplify the description, it is common to accept $\alpha$ as a constant between 0.5 and 2 (Åström and Canudas-de Wit, 2008; Olsson et al., 1998; Susanto et al., 2008). As seen in Figure $4, \dot{\varphi}_{s}$ changes the constant of the decay while $\alpha$ changes its curvature. Notice from Figures $4 \mathrm{a}$ and $4 \mathrm{~b}$ that small choices of $\alpha$ can considerably affect friction at high speeds. This is not desirable since the high speed effects should be described by the velocity strengthening function $h(\cdot)$. For these reasons, $\alpha$ is fixed as presented next. 


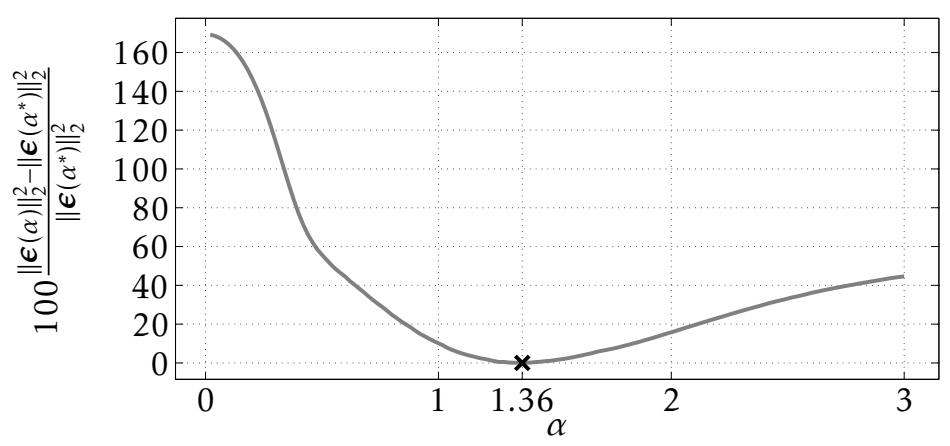

Figure 5: Relative cost increase as a function of $\alpha$ for $\mathcal{M}^{0}$.

Considering all friction data presented in this work, in a total of 488 friction curves with more than 5800 samples, $\alpha$ is chosen as the value minimizing Equation (3) for the model structure $\mathcal{M}^{0}$ when all other parameters are free at each friction curve. Figure 5 presents the resulting relative increase in the cost for different values of $\alpha$. The value with minimal cost gives $\alpha^{*}=1.36 \pm 0.011$.

\section{Empirically Motivated Modeling}

Using the friction estimation method described in Section 3.1, it is possible to design a set of experiments to analyze how the states $x^{*}$ affect friction. As shown in Section 3.2, the model structure $\mathcal{M}^{0}$ can represent the friction dependence on speed, $\dot{\varphi}$, fairly well. $\mathcal{M}^{0}$ is therefore taken as a primary choice, with $\alpha$ fixed at $\alpha^{*}=1.36$. Whenever a single instance of $\mathcal{M}^{0}$ can not describe the observed friction behavior, extra terms $\phi_{j}\left(\boldsymbol{x}^{*}, \boldsymbol{\theta}\right)$ are proposed and included in $\mathcal{M}^{0}$ to achieve a satisfactory model structure $\mathcal{M}^{*}$.

\subsection{Guidelines for the experiments}

It is important to isolate the influences of the different variables considered when modeling. The situation is particularly critical regarding temperature as it is difficult to control inside a robot joint. Moreover, due to the complex structure of an industrial robot, changes in joint angle might move the mass center of the robot arm system, causing variations of the load torques. To avoid undesired effects, the guidelines below were followed during the experiments.

\section{Isolating joint load torque dependency from joint angle dependency}

Using an accurate robot model ${ }^{\mathrm{i}}$, it is possible to predict the load torques at the joints for a given robot configuration (a set of all joints' angles). For example, Figure 6 shows the resulting $\tau^{\ell}$ and $\tau^{p}$ at joint two for configurations depending on the achievable angles for joints 2 and $4\left(\varphi_{2}^{a}\right.$ and $\left.\varphi_{4}^{a}\right)$. Using this information, $\mathrm{a}$

${ }^{\mathrm{i}} \mathrm{An}$ ABB internal tool was used for simulation purposes. 


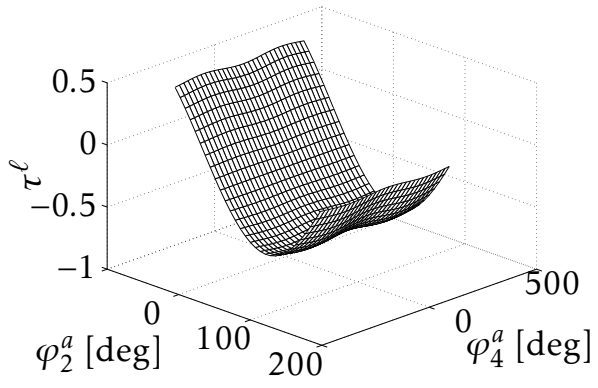

(a) Simulated $\tau^{\ell}$.

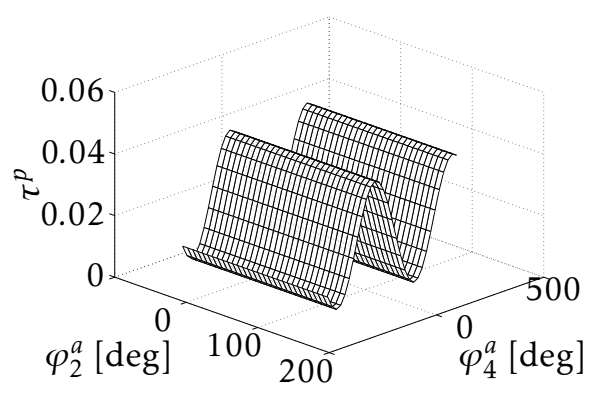

(b) Simulated $\tau^{p}$.

Figure 6: Simulated load torques at joint two caused by angle variations of joints 2 and $4, \varphi_{2}^{a}$ and $\varphi_{4}^{a}$ respectively. Notice the larger achievable range of values for $\tau^{\ell}$ compared to $\tau^{p}$.

set of configurations can be selected a priori in which it is possible to isolate these effects.

\section{Isolating temperature effects}

Using joint lubricant temperature measurements, the joint thermal decay constant $\kappa$ was estimated to $3.04 \mathrm{~h}$. By executing the friction curve estimation experiment periodically, for longer time than $2 \kappa$ (i.e. $>6.08 \mathrm{~h}$ ), the joint temperature is expected to have reached an equilibrium. Only data collected under an expected thermal equilibrium was considered for the analysis.

\subsection{Effects of joint angles}

Due to asymmetries in the contact surfaces, it has been observed that friction in rotating machines can depend on the angular position (Al-Bender and Swevers, 2008). It is therefore expected that this dependency occurs also in a robot joint. Following the experiment guidelines from the previous section, a total of 50 friction curves were estimated with variations of the joint angle in the range $\varphi^{a} \in(8.40,59.00)$ deg. As seen in Figure 7a, only small effects can be observed. The subtle deviations are comparable to the errors of the friction curve identified under constant values of $\left[\varphi^{a}, \tau^{p}, \tau^{\ell}, \xi\right]$. In fact, even a constant instance of $\mathcal{M}^{0}$ can describe the friction curves satisfactorily, no extra terms are thus required.

\subsection{Effects of load torques}

Since friction is related to the interaction between contacting surfaces, one of the first phenomenon observed was that friction varies according to the applied normal force. This can be explained by the increase of the true contact area between the surfaces under large normal forces. A similar reasoning can be extended to load torques in a revolute robot joint. Due to the elaborated gear- and bearing de- 


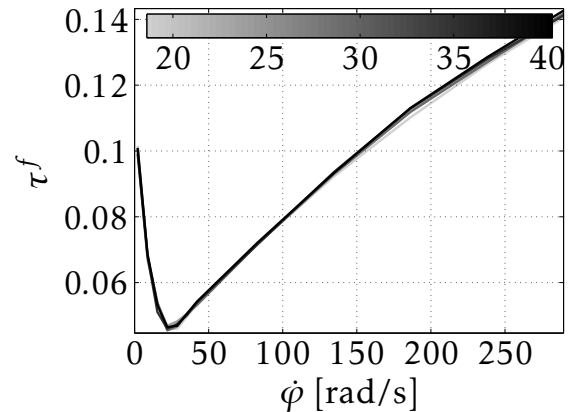

(a) Effects of $\varphi^{a}$ at $\tau^{\ell}=-0.39, \xi=34^{\circ} \mathrm{C}$.

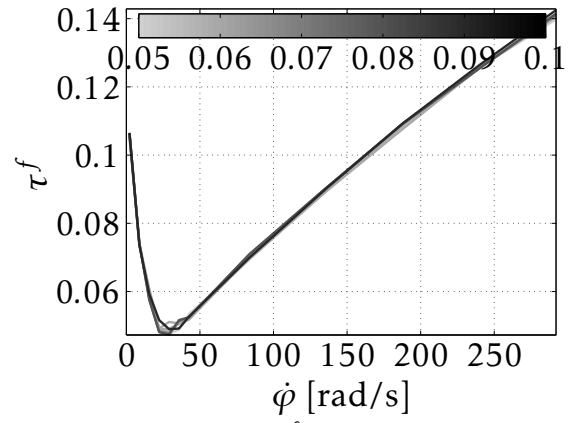

(b) Effects of $\tau^{p}$ at $\tau^{\ell}=-0.39, \xi=36^{\circ} \mathrm{C}$.

Figure 7: Friction curves for experiments related to $\varphi^{a}$ and $\tau^{p}$.

sign of the joint, it is also expected that torques in different directions will have different effects to the friction curve ${ }^{\mathrm{i}}$.

Only small variations of $\tau^{p}$, the perpendicular load torque, are achievable because of the mechanical construction of the robot, see Figure 6b. A total of 20 friction curves achieved at constant temperature were retrieved for joint two, where $\tau^{p}$ was varied in the range $\tau^{p} \in(0.04,0.10)$. As Figure $7 \mathrm{~b}$ shows, the influences of $\tau^{p}$ for the achievable range did not cause large changes to the friction curves. The model $\mathcal{M}^{0}$ is thus considered sufficient.

Large variations of $\tau^{\ell}$, the manipulation load torque, are possible by simply varying the arm configuration as seen in Figure 6a. A total of 50 friction curves were estimated where $\tau^{\ell}$ was varied over the range $\tau^{\ell} \in(-0.73,0.44)$. As seen in Figure 8 , the effects are significant.

Clearly, a single instance of $\mathcal{M}^{0}$ can not describe the observed phenomena. A careful analysis of the effects reveals that the main changes occur in the velocity weakening part of the curve. From Figure $8 \mathrm{c}$, it is possible to observe a linear bias-like $\left(f_{c}\right)$ increase and a linear increase of the standstill friction $\left(f_{s}\right)$ with $\left|\tau^{\ell}\right|$. Furthermore, as seen in Figure $8 \mathrm{~b}$, the Stribeck velocity $\dot{\varphi}_{s}$ is maintained fairly constant. The observations support an extension of $\mathcal{M}^{0}$ to

$$
\tau^{f}\left(\dot{\varphi}, \tau^{\ell}\right)=\left\{f_{c, 0}+f_{c, \ell}\left|\tau^{\ell}\right|\right\}+\left\{f_{s, 0}+f_{s, \ell}\left|\tau^{\ell}\right|\right\} e^{-\left|\frac{\dot{\varphi}}{\dot{\varphi}_{s, \ell}}\right|^{\alpha^{*}}}+f_{v} \dot{\varphi} .
$$

The model structure $\mathcal{M}^{\ell}$ is similar to the one presented in Hamon et al. (2010), where the changes in $f_{c}$ and $f_{s}$ appear as linear functions of $\left|\tau^{\ell}\right|$.

\subsection{Effects of temperature}

In lubricated mechanisms, the friction properties are related to both the thickness of the lubricant layer and its viscosity which, in turn, can vary with temper-

\footnotetext{
${ }^{\mathrm{i}}$ In fact, a full joint load description would require three torque and three force components.
} 


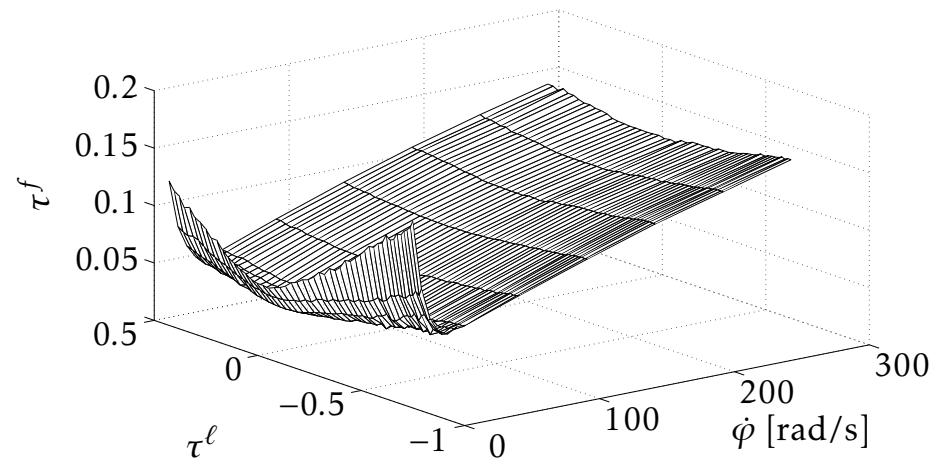

(a) Friction levels as a function of $\dot{\varphi}$ and $\tau^{\ell}$.

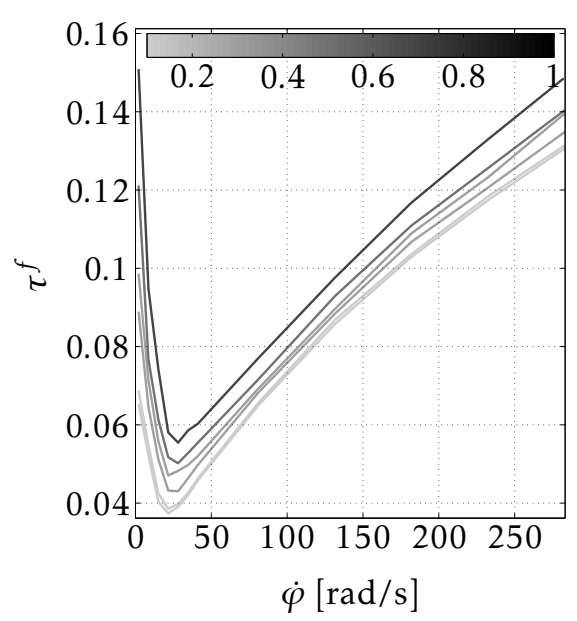

(b) Friction levels as a function of $\dot{\varphi}$ for different values of $\tau^{\ell}$.

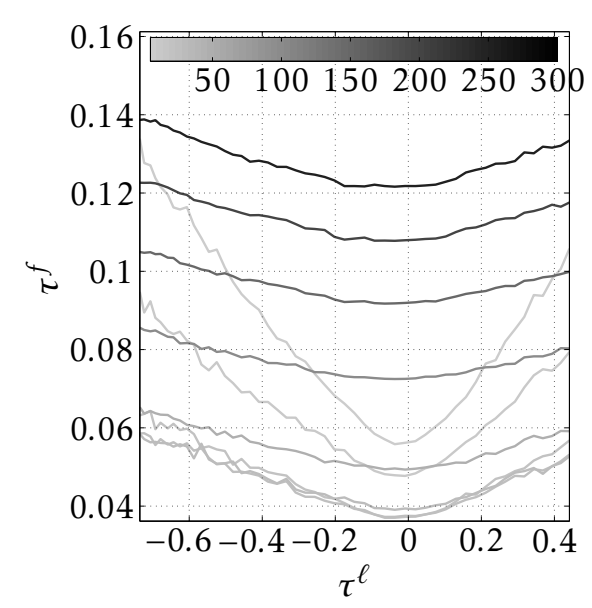

(c) Friction levels as a function of $\tau^{\ell}$ for different values of $\dot{\varphi}$.

Figure 8: The dependencies of friction with the manipulation load torque, $\tau^{\ell}$, at $\xi=34^{\circ} \mathrm{C}$. 
ature (Seeton, 2006). Dedicated experiments were made to analyze the effects of temperature. At first, the joint was warmed up to $81.2^{\circ} \mathrm{C}$ by running the joint continuously back and forth. Then, while the robot cooled, 50 friction curves were retrieved with variations of $\xi$ over the range $\xi \in(38.00,81.20)^{\circ} \mathrm{C}$. In order to resolve combined effects of $\xi$ and $\tau^{\ell}$, two manipulation torque levels were used, $\tau^{\ell}=-0.02$, and $\tau^{\ell}=-0.72$. As it can be seen in Figure 9 , the effects of $\xi$ are significant.

Temperature has an influence on both velocity regions of the friction curves. In the velocity-weakening region, a linear increase of the standstill friction $\left(f_{s}\right)$ with temperature can be observed according to Figure 9b. In Figure 9c, it can be seen that the Stribeck velocity $\left(\dot{\varphi}_{s}\right)$ increases linearly with temperature. The effects in the velocity-strengthening region appear as a nonlinear, exponential-like, decrease of the velocity-dependent slope $\left(f_{v}\right)$, as seen in Figures $9 \mathrm{~b}$ and $9 \mathrm{c}$.

Combined effects of $\tau^{\ell}$ and $\xi$

It is also important to study possible combined effects of load and temperature. To visualize possible co-effects, the friction surfaces in Figure 9a are subtracted from each other, yielding $\widetilde{\tau}^{f}$. As it can be seen in Figure 10a, the result is fairly temperature independent. This can be interpreted as an indication that the variables are additively separable, i.e. they appear in separate additive terms. Under this modeling assumption, it is possible to subtract the $\tau^{\ell}$-effects from the surfaces in Figure 9a and solely obtain temperature related phenomena. The previously proposed terms to describe the $\tau^{\ell}$-effects in $\mathcal{M}^{\ell}$ were

$$
\tau^{f}\left(\tau^{\ell}\right)=f_{c, \ell}\left|\tau^{\ell}\right|+f_{s, \ell}\left|\tau^{\ell}\right| e^{-\left|\frac{\dot{\varphi}}{\dot{\varphi}_{s}}\right|^{\alpha^{*}}} .
$$

The parameters for this model were found from an identification of model $\mathcal{M}^{\ell}$ using the data set from Figure 8. With the achieved parameters, the computed effects of the load $\tau^{f}\left(\tau^{\ell}\right)$ were subtracted from the friction data of Figure 9 a, i.e., the quantities $\tau^{f}-\widehat{\tau}^{f}\left(\tau^{\ell}\right)$ were computed. The resulting friction levels are shown in Figure 10b. As can be seen, the resulting friction levels become very similar, independent of the manipulation load torques used. This further supports the modeling assumption that the effects of temperature and load are additively separable. Even after removing the load effects, the original model structure cannot fully characterize all observed phenomena and new terms should be added to describe the temperature effects. 


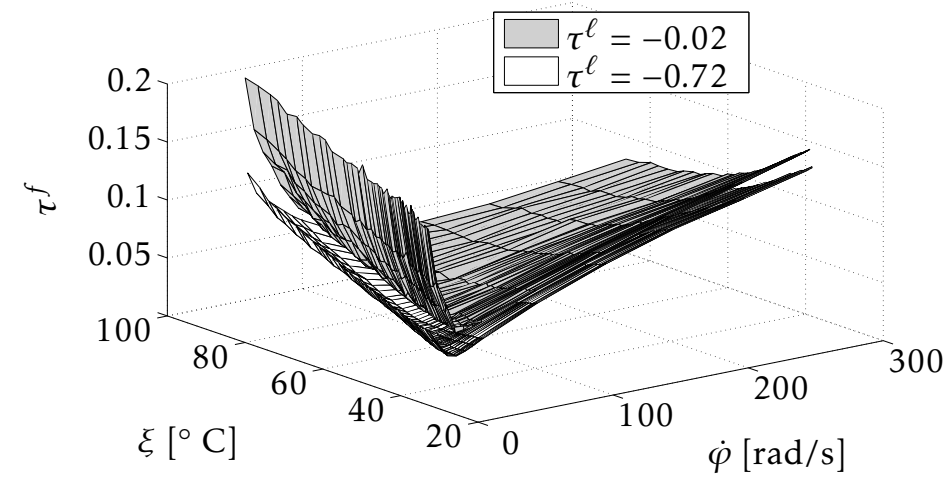

(a) Friction levels as a function of $\dot{\varphi}$ and $\xi$ for two different levels of $\tau^{\ell}$.

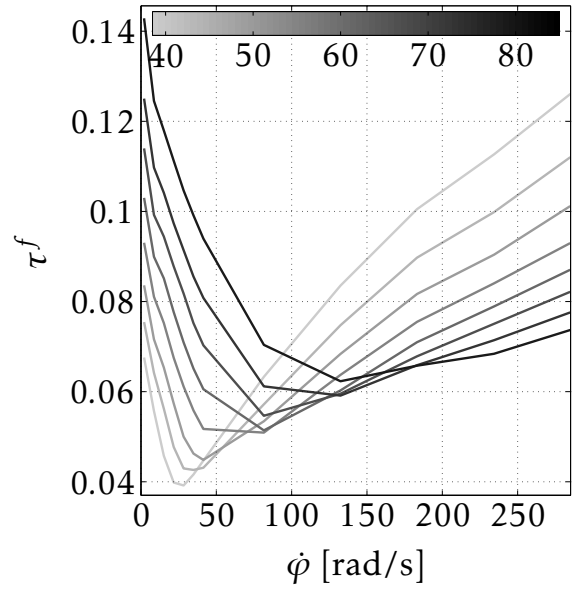

(b) Friction levels as a function of $\dot{\varphi}$ for different values of $\xi$ at $\tau^{\ell}=-0.02$.

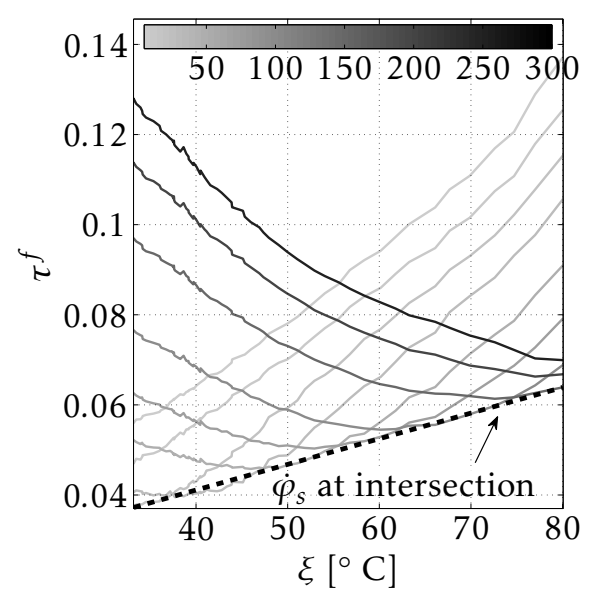

(c) Friction levels as a function of $\xi$ for different values of $\dot{\varphi}$ at $\tau^{\ell}=-0.02$.

Figure 9: The dependencies of friction with temperature, $\xi$. 


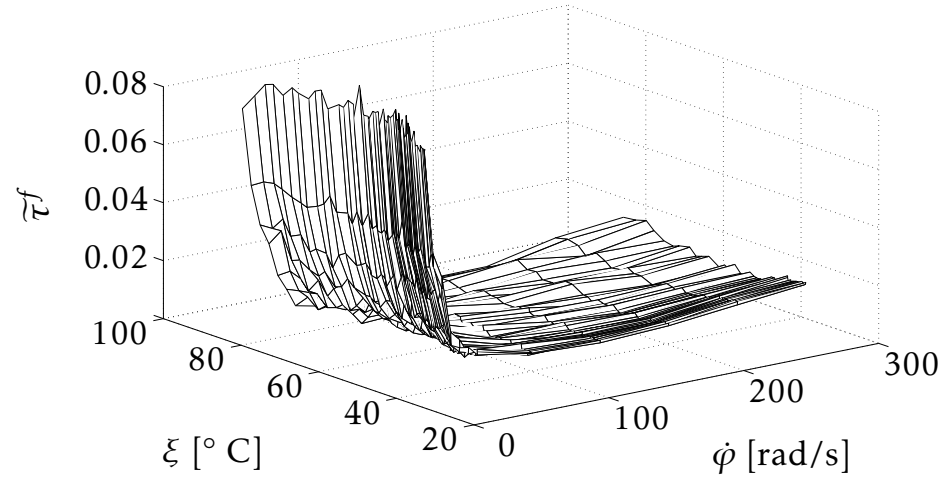

(a) Difference $\widetilde{\tau}^{f}$ between the friction data in Figure 9a.

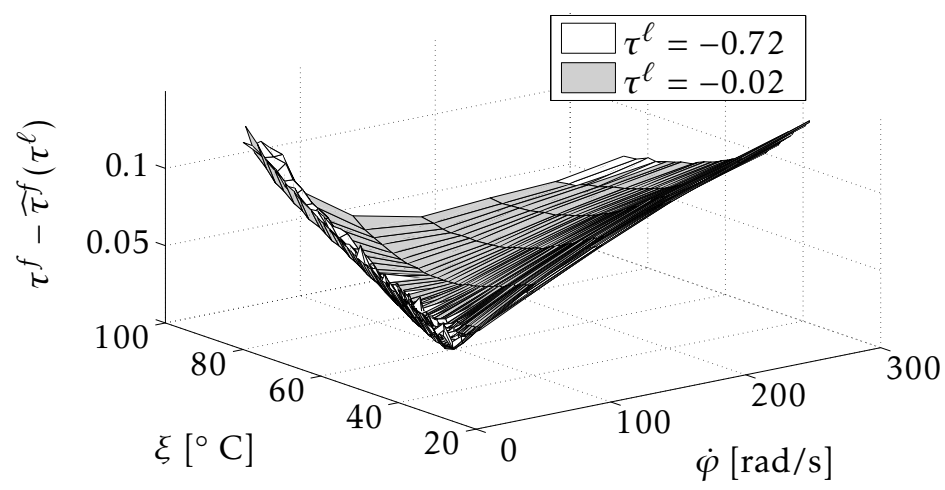

(b) Friction data of Figure 9a after subtraction of the $\tau^{\ell}$ dependent terms.

Figure 10: Indication that the effects of $\xi$ and $\tau^{\ell}$ are additively separable. 
Table 2: Identified parameters for $\mathcal{M}^{*}$.

\begin{tabular}{cccc}
\hline$f_{c, 0}\left[10^{-2}\right]$ & $f_{c, \ell}\left[10^{-2}\right]$ & $f_{s, \ell}\left[10^{-1}\right]$ & $\dot{\varphi}_{s, \ell}$ \\
$3.11 \pm 0.028$ & $2.34 \pm 0.071$ & $1.26 \pm 0.025$ & $9.22 \pm 0.12$ \\
\hline$f_{s, 0}\left[10^{-2}\right]$ & $f_{s, \xi}\left[10^{-3}\right]$ & $\dot{\varphi}_{s, 0}$ & $\dot{\varphi}_{s, \xi}$ \\
$-2.50 \pm 0.12$ & $1.60 \pm 0.022$ & $-24.81 \pm 0.87$ & $0.98 \pm 0.018$ \\
\hline$f_{v, 0}\left[10^{-4}\right]$ & $f_{v, 0}\left[10^{-4}\right]$ & $\xi_{\mathrm{Vo}}$ & $\alpha^{*}$ (fixed) \\
$1.30 \pm 0.056$ & $1.30 \pm 0.056$ & $20.71 \pm 0.91$ & 1.36 \\
\hline
\end{tabular}

\subsection{A proposal for $\mathcal{M}^{*}$}

From the characteristics of the $\xi$-related effects and the already discussed $\tau^{\ell}$ effects, $\mathcal{M}^{\ell}$ is extended to:

$$
\begin{aligned}
\tau^{f}\left(\dot{\varphi}, \tau^{\ell}, \xi\right) & =\left\{f_{c, 0}+f_{c, \ell}\left|\tau^{\ell}\right|\right\}+f_{s, \ell}\left|\tau^{\ell}\right| e^{-\left|\frac{\dot{\varphi}}{\dot{\varphi}_{s, \ell}}\right|^{\alpha^{*}}} & \left(\mathcal{M}_{g, \ell}^{*}\right) \\
& +\left\{f_{s, 0}+f_{s, \xi} \xi\right\} e^{-\left|\frac{\dot{\varphi}}{\left\{\dot{\varphi}_{s, 0}+\dot{\varphi}_{s, \xi \xi}\right.}\right|^{\alpha^{*}}} & \left(\mathcal{M}_{g, \xi}^{*}\right) \\
& +\left\{f_{v, 0}+f_{v, \xi} e^{\frac{-\xi}{\xi_{\mathrm{V}}}}\right\} \dot{\varphi} . & \left(\mathcal{M}_{h, \xi}^{*}\right)
\end{aligned}
$$

In the above equation, the parameters are written with subscripts ${ }_{-0}, \ell$ or $\_\xi$ in order to clarify its origin related to $\mathcal{M}^{0}, \tau^{\ell}$ or $\xi$. The first $\mathcal{M}_{g}^{*}$ expressions relate to the velocity-weakening friction while $\mathcal{M}_{h}^{*}$ relates to the velocity-strengthening regime. The load $\tau^{\ell}$ only affects the velocity-weakening regime and requires a total of three parameters, $\left[f_{c, \ell}, f_{s, \ell}, \dot{\varphi}_{s, \ell}\right]$. The temperature $\xi$ affects both regimes and requires four parameters, $\left[f_{s, \xi}, \dot{\varphi}_{s, \xi}, f_{v, \xi}, \xi_{V_{0}}\right]$. The four remaining parameters, $\left[f_{c, 0}, f_{s, 0}, \dot{\varphi}_{s, 0}, f_{v, 0}\right]$, relate to the original friction model structure $\mathcal{M}^{0}$. Notice that under the modeling assumption that $\tau^{\ell}$ - and $\xi$ effects are additively separable, their respective expressions appear as separated sums in $\mathcal{M}^{*}$.

The term $f_{v, \xi} e^{-\xi / \xi_{\text {Vo }}}$ in $\mathcal{M}_{h, \xi}^{*}$ is motivated by the exponential-like behavior of viscous friction (recall Figure 9c). In fact, the parameter $\xi_{V_{0}}$ is a reference to the Vogel-Fulcher-Tamman exponential description of viscosity and temperature. This description is valid for the temperature range considered here and more complex expressions may be needed for larger temperature variations, see e.g. Seeton (2006).

\subsection{Validation}

The parameters for the proposed model are identified based on friction data from Figures 8 and 9 and are presented in Table 2. A separate data set is used for the validation of the proposed model structure $\mathcal{M}^{*}$. It consists of several friction curves retrieved at different $\tau^{\ell}$ - and $\xi$ values, as seen in Figure 11 . The distribution of the prediction errors, $p(\epsilon)$, achieved with the validation data set is shown in Figure 12. For a comparison, the distribution of the errors related to an instance of $\mathcal{M}^{0}$ with parameters given in Table 1 is also shown. As it can be seen, 


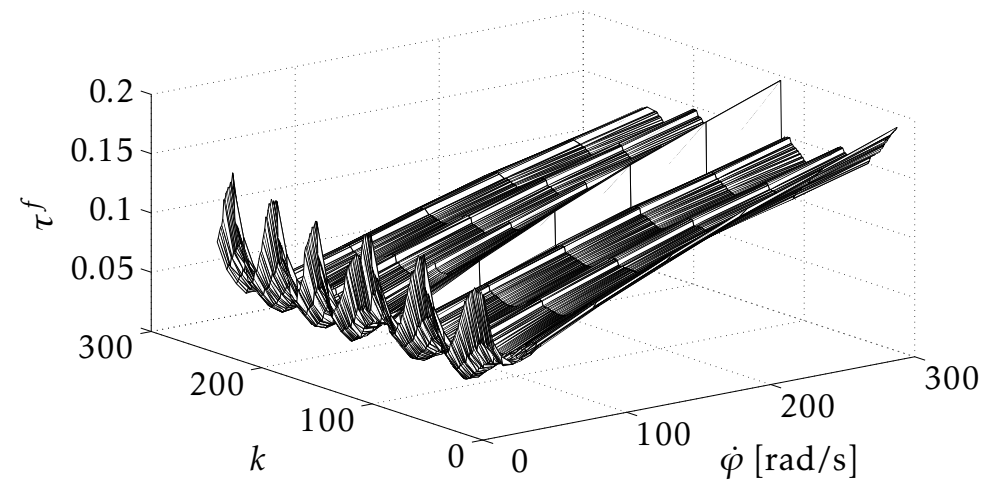

(a) Friction data used for validation.

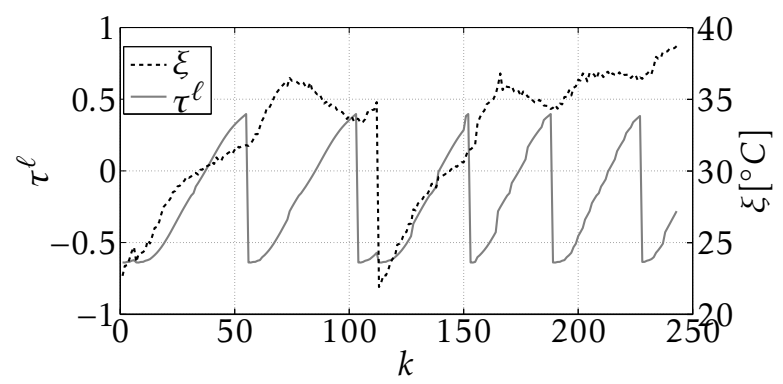

(b) $\tau^{\ell}$ - and $\xi$ conditions used in each friction curve.

Figure 11: Validation data set. Notice the large variations of $\xi$-and $\tau^{\ell}$ in (b) when registering the friction curves in (a).

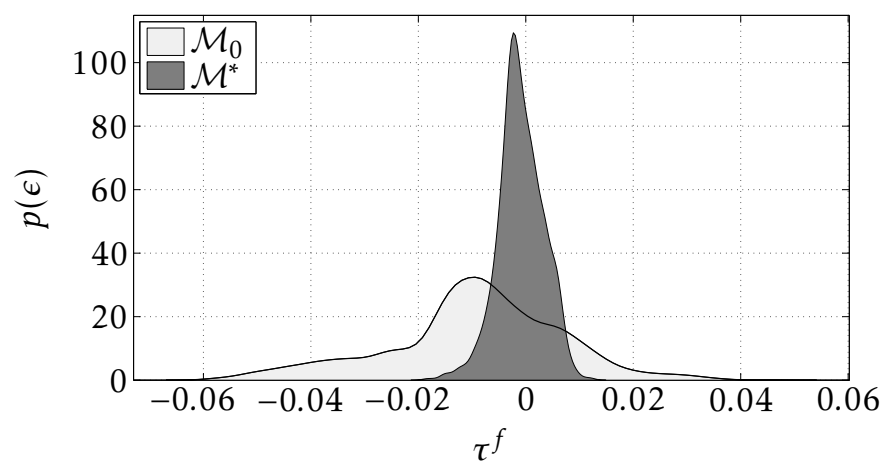

Figure 12: Distribution of the prediction errors for the models $\mathcal{M}^{0}$ and $\mathcal{M}^{*}$ achieved using the validation data set. Notice the considerable improved performance for $\mathcal{M}^{*}$. 
$\mathcal{M}^{*}$ is able to capture considerably more of the friction behavior than $\mathcal{M}^{0}$, with only speed dependence. The mean, standard deviation and largest absolute error for $\mathcal{M}^{*}$ are respectively $-9.2410^{-4}, 4.2310^{-3}, 1.8810^{-2}$, compared to $1.0910^{-2}$, $1.3410^{-2}, 7.5810^{-2}$ for $\mathcal{M}^{0}$. The proposed model structure has also been successfully validated in other joints with similar gearboxes, but it might be interesting to validate it in other robot types and even other types of rotating mechanisms.

\section{Conclusions and Further Research}

The main contribution of this paper is the empirical analysis of the effects of position, speed, load and temperature to friction in a robot joint and the proposed model to describe the most significant effects of speed, load and temperature. As shown in the validation results, a model that includes a description of load and temperature might be needed for a more accurate representation of friction.

In the studies, the friction phenomena were fairly direction independent. If this was not the case, two instances of the proposed model could be used to describe the whole speed range, but requiring two times more parameters. The proposed model has a total of 11 parameters, four of those enter the model in a nonlinear fashion. The identification of such a model is computationally costly and requires data from several different operating conditions. Studies on defining sound identification excitation routines are therefore important.

Only steady-state friction (measured when transients caused by velocity changes have disappeared) was considered in the studies. It would be interesting to investigate if a dynamic model, for instance given by the LuGre model structure, could be used to describe dynamic friction with extensions from the proposed model. This task presents practical experimental challenges and should perhaps be performed in a robot joint mounted in a test bench instead of on a robot arm system which has complex dynamics.

A practical limitation of the proposed model is the requirement on availability of load torque and temperature estimates. Up to date, torque- and joint temperature sensors are not available in standard industrial robots. As mentioned in Section 4.1 , the joint torque components can be estimated from the torque reference to the drive system by means of an accurate robot model. In this situation, it is important to have correct load parameters in the model to calculate the components of the load torques.

Despite these experimental challenges, there is a great potential for the use of the proposed model for simulation-, design- and evaluation purposes. The designer of control algorithms, the diagnosis engineer, the gearbox manufacturer, etc. would benefit with the use of more realistic friction models. 


\section{Bibliography}

F. Al-Bender and J. Swevers. Characterization of friction force dynamics. IEEE Control Systems Magazine, 28(6):64-81, 2008.

B. Armstrong-Hélouvry. Control of Machines with Friction. Springer, 1991.

K. J. Åström and C. Canudas-de Wit. Revisiting the LuGre friction model. IEEE Control Systems Magazine, 28(6):101-114, December 2008.

A. C. Bittencourt, E. Wernholt, S. Sander-Tavallaey, and T. Brogårdh. An extended friction model to capture load and temperature effects in robot joints. In Proceedings of the 2010 IEEE/RSJ International Conference on Intelligent Robots and Systems, pages 6161-6167, Taipei, Taiwan, October 2010.

A. C. Bittencourt, P. Axelsson, Y. Jung, and T. Brogårdh. Modeling and identification of wear in a robot joint under temperature disturbances. In Proceedings of the 18th IFAC World Congress, volume 18, Milan, Italy, August 2011.

P. J. Blau. Embedding wear models into friction models. Tribology Letters, 34(1), April 2009.

L. C. Bo and D. Pavelescu. The friction-speed relation and its influence on the critical velocity of stick-slip motion. Wear, 82(3):277-289, 1982.

B. Bona and M. Indri. Friction compensation in robotics: an overview. In Proceedings of the 44th IEEE International Conference on Decision and Control, Seville, Spain, December 2005.

D. Brambilla, L. Capisani, A. Ferrara, and P. Pisu. Fault detection for robot manipulators via second-order sliding modes. IEEE Transactions on Industrial Electronics, 55(11):3954-3963, November 2008.

F. Caccavale, P. Cilibrizzi, F. Pierri, and L. Villani. Actuators fault diagnosis for robot manipulators with uncertain model. Control Engineering Practice, 17(1): 146-157, 2009.

M. Dohring, E. Lee, and W. Newman. A load-dependent transmission friction model: theory and experiments. In Proceedings of the 1993 IEEE International Conference on Robotics and Automation, pages 430-436, Atlanta, USA, May 1993.

D. Dowson. History of Tribology. Professional Engineering Publishing, London, UK., 2nd edition, 1998.

B. Freyermuth. An approach to model based fault diagnosis of industrial robots. In Proceedings of the 1991 IEEE International Conference on Robotics and Automation, volume 2, pages 1350-1356, April 1991.

A. Gogoussis and M. Donath. Coulomb friction effects on the dynamics of bearings and transmissions in precision robot mechanisms. In Proceedings of the 
1998 IEEE International Conference on Robotics and Automation, pages 14401446, Leuven, Belgium, April 1988.

G. H. Golub and V. Pereyra. The differentiation of pseudo-inverses and nonlinear least squares problems whose variables separate. SIAM Journal on Numerical Analysis, 10(2):413-432, April 1973.

Y. Guo, Z. Qu, Y. Braiman, Z. Zhang, and J. Barhen. Nanotribology and nanoscale friction. IEEE Control Systems Magazine, 28(6):92-100, December 2008.

P. Hamon, M. Gautier, and P. Garrec. Dynamic identification of robots with a dry friction model depending on load and velocity. In Proceedings of the 2010 IEEE/RSJ International Conference on Intelligent Robots and Systems, pages 6187-6193, Taipei, Taiwan, October 2010.

A. Harnoy, B. Friedland, and S. Cohn. Modeling and measuring friction effects. IEEE Control Systems Magazine, 28(6), December 2008.

B. Jacobson. The Stribeck memorial lecture. Tribology International, 36(11):781789, 2003.

H. M. Kim, S. H. Park, and S. I. Han. Precise friction control for the nonlinear friction system using the friction state observer and sliding mode control with recurrent fuzzy neural networks. Mechatronics, 19(6):805-815, 2009.

L. Ljung. System Identification: Theory for the User. Prentice Hall PTR, 2nd edition, December 1999.

R. Mattone and A. D. Luca. Relaxed fault detection and isolation: An application to a nonlinear case study. Automatica, 42(1):109 - 116, 2009.

M. McIntyre, W. Dixon, D. Dawson, and I. Walker. Fault identification for robot manipulators. IEEE Transactions on Robotics, 21(5):1028-1034, October 2005.

M. Namvar and F. Aghili. Failure detection and isolation in robotic manipulators using joint torque sensors. Robotica, 28(4):549-561, 2010.

H. Olsson, K. J. Åström, C. C. de Wit, M. Gafvert, and P. Lischinsky. Friction models and friction compensation. European Journal of Control, 4(3):176-195, 1998.

C. J. Seeton. Viscosity-temperature correlation for liquids. Tribology Letters, 22 (1):67-78, March 2006.

W. Susanto, R. Babuska, F. Liefhebber, and T. van der Weiden. Adaptive friction compensation: application to a robotic manipulator. In Proceedings of 17th IFAC World Congress, Seoul, South Korea, December 2008.

A. Tustin. The effects of backlash and of speed-dependent friction on the stability of closed-cycle control systems. Journal of the Institution of Electrical Engineers - Part IIA: Automatic Regulators and Servo Mechanisms, 94(1):143151, May 1947. 
A. T. Vemuri and M. M. Polycarpou. A methodology for fault diagnosis in robotic systems using neural networks. Robotica, 22(04):419-438, 2004.

R. Waiboer. Dynamic Modelling, Identification and Simulation of Industrial Robots. PhD thesis, University of Twente, 2007.

R. Waiboer, R. Aarts, and B. Jonker. Velocity dependence of joint friction in robotic manipulators with gear transmissions. In Proceedings of the 2005 ECCOMAS Thematic Conference Multibody Dynamics, pages 1-19, Madrid, Spain, 2005.

M. Woydt and R. Wäsche. The history of the Stribeck curve and ball bearing steels: The role of Adolf Martens. Wear, 268(11-12):1542-1546, 2010. 


\section{Paper B}

\section{Modeling and Experiment Design for Identification of Wear in a Robot Joint under Load and Temperature Uncertainties based on Friction Data}

Authors:

André Carvalho Bittencourt and Patrik Axelsson.

Edited version of the paper:

A. C. Bittencourt and P. Axelsson. Modeling and experiment design for identification of wear in a robot joint under load and temperature uncertainties based on friction data. IEEE/ASME Transactions on Mechatronics, 19(5):1694-1706, October 2014.

Parts of this paper were previously published in:

A. C. Bittencourt, P. Axelsson, Y. Jung, and T. Brogårdh. Modeling and identification of wear in a robot joint under temperature disturbances. In Proceedings of the 18th IFAC World Congress, volume 18, Milan, Italy, August 2011. 



\title{
Modeling and Experiment Design for Identification of Wear in a Robot Joint under Load and Temperature Uncertainties based on Friction Data
}

\author{
André Carvalho Bittencourt and Patrik Axelsson \\ Dept. of Electrical Engineering, \\ Linköping University, \\ SE-581 83 Linköping, Sweden \\ \{andrecb, axelsson\} @isy.liu.se
}

\begin{abstract}
The effects of wear to friction are studied based on constant-speed friction data collected from dedicated experiments during accelerated wear tests. It is shown how the effects of temperature and load uncertainties produce larger changes to friction than those caused by wear, motivating the consideration of these effects. Based on empirical observations, an extended friction model is proposed to describe the effects of speed, load, temperature and wear. Assuming availability of such model and constant-speed friction data, a maximum likelihood wear estimator is proposed. The performance of the wear estimator under load and temperature uncertainties is found by means of simulations and verified under three case studies based on real data. Practical issues related to experiment length are considered based on a criterion for optimal selection of speed points to collect friction data that minimizes the mean square estimation error for any unbiased wear estimator. As it is shown, reliable wear estimates can be achieved even under load and temperature uncertainties, making condition based maintenance of industrial robots possible.
\end{abstract}

\section{Introduction}

Industrial robots are used as a key factor to improve productivity, quality and safety in automated manufacturing. Robot installations are many times of crucial importance in the processes they are used and an unexpected robot stop or malfunction may lead to production and economical losses. Increased safety, reliability, availability and maintainability (SRAM) are therefore critical for industrial robots. Preventive scheduled maintenance is a common approach to guarantee the requirements on SRAM in the manufacturing industry. Such scheduling is 
often determined from the estimated lifespan of robot components, with considerable margins. Because preventive maintenance is not determined by the actual robot condition, unnecessary maintenance actions might take place when utilizing this strategy.

In the current scenario, maintainability of industrial robots can be greatly improved with the use of methods to determine its condition, allowing for condition based maintenance (СВM). With focus on service, it is important that a change in condition is detected before a critical degradation takes place, so that timely maintenance actions can take place. Wear in a robot joint may lead to a degradation of performance and to an eventual failure. Because wear typically develops slowly with time and usage, it might be detectable in an early stage, making CBM possible.

According to Lansdown et al. (1987) wear can be defined as "the progressive loss of material from the operating surface of a body occurring as a result of relative motion at its surface". The need for relative motion between surfaces implies that the wear mechanisms are related to mechanical action between surfaces. This is an important distinction to other processes with a similar outcome and very different nature, e.g. corrosion (Williams, 2005). Wear is naturally related to friction since friction can be defined as the tangential reaction force between two surfaces in contact. Friction opposes motion, dissipating kinetic energy. A part of the work produced by friction appears as heat transfer, vibrations and acoustic emissions. Other outcomes of friction are plastic deformation, adhesion and fracture which can relate to wear.

The accumulated wear in a tribosystem may lead to variations in friction (Kato, 2000; Bittencourt et al., 2011). Alternatives for wear monitoring are thus possible provided it is applicable to observe friction and the relation between friction and wear is known. Monitoring friction to infer about wear is however challenging since friction is significantly affected by other factors such as temperature and load. The effects of temperature are specially difficult since temperature is not measured in typical robot applications. These co-effects should nevertheless be considered when verifying the reliability of a solution.

In the literature, little can be found about wear estimation for industrial robots. This may be attributed to the lack of wear models available and the high costs and time required to perform wear experiments. There are related approaches used for fault detection, where the objective is to decide whether a change from nominal is present. Faults are typically considered as actuator malfunctions, modeled as changes in the output torque signals or in the parameters of a robot model. The latter includes the case of friction changes, which is important since they can relate to wear.

Considering the nonlinear nature of a manipulator, the use of nonlinear observers is a common approach for fault detection. Different design approaches have been suggested, see e.g. Filaretov et al. (1999); McIntyre et al. (2005); Caccavale et al. 
(2009); Guo et al. (2012); Dixon et al. (2000); De Luca and Mattone (2003), and the observer stability is typically guaranteed by analysis of the decay rate of a candidate Lyapunov function. Due to uncertainties in the modeling assumptions, approaches have been suggested to improve robustness of observer-based solutions. In Brambilla et al. (2008); De Luca and Mattone (2003); Guo et al. (2012), nonlinear observers are used together with adaptive schemes while in Caccavale et al. (2009), support vector machines are trained to model the uncertainties. A nonlinear fault observer is suggested by Vemuri and Polycarpou (2004) based on a neural network model for the abnormal robot behavior and defines a robust adaptation rule based on known bounds for the uncertainties. In Ray et al. (2001), an observer is used to estimate friction torques in a rotating machine; the presence of a friction change is detected based on a multiple hypothesis test where each hypothesis is associated to a known friction model.

In Chen (2011), the passivity property of Lagrangian systems is used to define energy balance equations which are monitored for fault detection and isolation; the framework is illustrated with a simulation study of a robot manipulator with faults in dissipative components (e.g. friction changes) and energy-storing components (e.g. load changes). Because the energy balance is also affected by disturbances, knowledge of these effects to the system's energy can be used to achieve robustness; some approaches are discussed in Chen (2011), see also Marton (2012).

The vibration patterns generated from a robot joint also contain valuable information about its condition. In Eski et al. (2011), neural networks are used to learn the vibration patterns of a robot based on accelerometers' measurements. Similarly, the acoustic emissions of the robot joints may change under a fault. In Olsson et al. (2004), features of sound measurements, i.e. peaks of a wavelet transformation, are monitored and determination of a fault is achieved based on labeled data using a nearest neighbor classifier. Besides the extra sensors needed, these approaches require data from a pre-defined trajectory.

The estimation of friction parameters in a robot model from measured data is a natural approach for fault detection because of the physical interpretation of these parameters. In Freyermuth (1991), estimates of the Coulomb and viscous friction parameters are compared to confidence values of their nominal behavior. In the experimental study presented, these parameters could indicate some of the faults but could not readily distinguish between them; e.g. the increase of joint temperature had a similar effect as a fault in the drive-chain. The friction model used in Freyermuth (1991) did not consider the effects of temperature which, as illustrated here, can be larger than those caused by wear. The relation between temperature and friction was considered in Marton and van der Linden (2012), where estimates of the viscous friction parameter are used to monitor the lubricant health in a mechanical transmission. The lubricant temperature is estimated based on a Kalman filter using environment temperature measurements and a heat transfer model. A similar approach but based on an observer of the viscous friction torque is also presented in Marton (2011) with simulation studies for a robot joint. 
In this paper, the effects of wear to friction are studied based on empirical observations. By introducing a variable to describe the effects of wear to friction, an extended friction model is proposed to describe wear-related effects. The suggested friction model is used to define a maximum-likelihood wear estimator based on constant-speed friction data which are collected from dedicated experiments, in an off-line manner. Off-line solutions will decrease the robot availability which is undesirable. The trade-off between experiment length and the estimator accuracy is therefore important and is studied in detail. The main contributions leading to the proposed solutions are listed

- first, the effects of wear to friction are modeled based on empirical observations;

- an extended friction model describing the effects of speed, temperature, load and wear is proposed and identified;

- with a known friction model, maximum likelihood wear estimators are proposed;

- experiment design is considered based on the achievable performance for any unbiased wear estimator;

- the estimator is validated through simulations and case studies based on real data.

These results are presented through Sections 2.3 to 5. Sections 2.1 and 2.2 review earlier results presented in Bittencourt and Gunnarsson (2012) which are used in this paper; namely, an experiment routine used to provide constant-speed friction data and a friction model to describe the nominal behavior of friction, i.e. under no significant presence of wear. The conclusions and proposals for further research are presented in Section 6. The studies presented in the paper are based on observed friction for joint two of ABB IRB 6620 industrial robots. Joint two is chosen for the study as it endures great stress variations for the type of robot considered. The joint is equipped with a rotary vector type of gearbox which is commonly found in industrial robots of similar sizes.

A preliminary version of a wear estimation approach based on constant-speed friction data was presented in Bittencourt et al. (2011) where the wear model was first presented and a wear estimator was suggested and verified. Here, wear estimators are suggested based on a statistical framework, with a more in-depth study of experiment design, achievable performance and verification studies.

\section{Steady-State Friction in a Robot Joint}

Friction is a dynamic phenomenon; at a contact level, the surfaces' asperities can be compared to (very stiff) bristles in a brush, each of which can be seen as a body with its own dynamics connected by the same bulk (Al-Bender and Swevers, 2008; De Moerlooze et al., 2010). Because the internal friction states are not measurable, it is common to study friction in steady-state, when friction presents a static behavior. As presented in Bittencourt and Gunnarsson (2012), experimental data show that friction data collected under constant speed can be described 
by a static nonlinear function.

The simplified behavior of steady-state friction ${ }^{\mathrm{i}}$ facilitates the modeling task and the determination of the source of changes to friction, e.g. caused by wear or temperature. A shortcoming is that constant-speed data are not readily available from a robot's normal operation. This type of data can however be collected based on the experimental procedure described in Bittencourt and Gunnarsson (2012). Data collected from such an experiment will be used as input to the wear estimators suggested here and the procedure is briefly described in Section 2.1. Using constant-speed friction data, the behavior of friction is studied in detail in Bittencourt and Gunnarsson (2012) where a static nonlinear model was suggested to describe the effects of speed, temperature and load. This model is reviewed in Section 2.2 and extended in Sections 2.3 and 2.4 to include a description of observed effects caused by wear.

\subsection{A procedure to estimate friction at a fixed speed level}

A manipulator is a multivariable, nonlinear system that can be described in a general manner through the rigid multi-body dynamic model

$$
M(\varphi) \ddot{\varphi}+C(\varphi, \dot{\varphi}) \dot{\varphi}+\tau^{g}(\varphi)+\tau^{f}=\tau
$$

where $\varphi$ is the vector of motor position, $M(\varphi)$ is the inertia matrix, $C(\varphi, \dot{\varphi})$ relates to Coriolis and centrifugal terms, $\tau^{g}(\varphi)$ are the gravity-induced joint torques and $\tau^{f}$ contains the joint friction components. The system is controlled by the input torque, $\tau$, applied by the motors (in the experiments the torque reference from the servo was measured) ${ }^{\mathrm{ii}}$. For single joint movements (so that centrifugal forces are zero at that joint) and under constant speed (so that inertial torques are zero), the applied torque at the joint under actuation drives only gravity induced torques and friction, i.e.

$$
\tau^{g}(\varphi)+\tau^{f}=\tau
$$

By considering forward and backward movements for a speed level $\overline{\dot{\varphi}}$ around a configuration $\bar{\varphi}$ (so that $\tau^{g}(\bar{\varphi})$ is the same in both directions), a direction independent estimate of friction can be achieved as

$$
\tau^{f}=\left(\tau^{+}-\tau^{-}\right) / 2
$$

where $\tau^{+}$and $\tau^{-}$are the resulting torques when the joint is moved forwards, respectively backwards. In the experiments, each joint is moved separately with the desired speed $\overline{\dot{\varphi}}$ in both directions around a given joint angle $\bar{\varphi}$. As an example,

\footnotetext{
${ }^{\mathrm{i}}$ In this paper, the term steady-state friction is used as a synonym of the friction observed in constant-speed conditions.

${ }^{\mathrm{ii}}$ It is known that using the torque reference from the controller as a measure of the joint torque might not always hold. The torque controller for the robot considered in the studies has a steadystate error of maximum 5\%. The variations are because of the temperature dependence of the torque constant of the motors.
} 


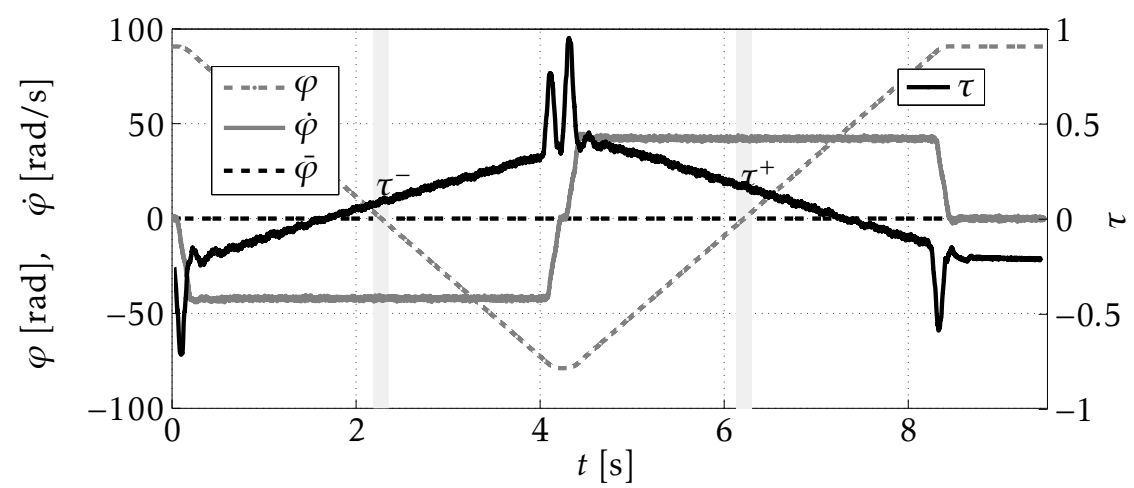

(a) Data collected for the estimation of the friction level at $\overline{\dot{\varphi}}=42$ and $\bar{\varphi}=0$.

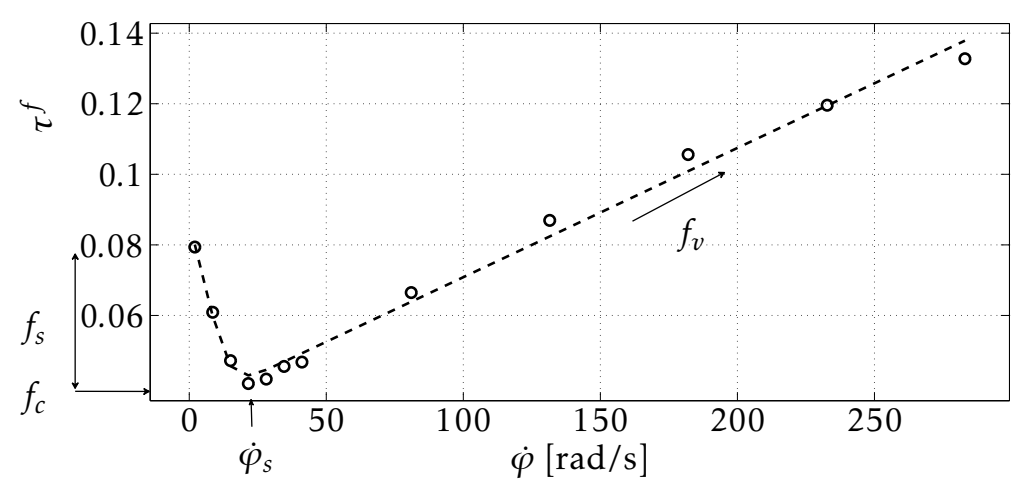

(b) Estimated friction levels (circles) and predictions based on (4).

Figure 1: Experimental procedure for the estimation of constant-speed friction. Data are collected for single joint movements back and forth around a position $\bar{\varphi}$ for a desired speed $\dot{\dot{\varphi}}$, as shown in (a). The constant speed torque levels for the forward and backward movements, $\tau^{+}$and $\tau^{-}$respectively, are segmented and used for estimation of $\tau^{f}$ according to (3). The procedure can be repeated for different speed levels and plotted against speed in a friction curve as shown by the circles in (b). The dashed line in (b) corresponds to predictions computed based on the model in (4) with an interpretation of the model parameters. 
Figure 1a shows the measured joint angle-, speed- and torque ${ }^{i}$ data generated from such experiment in joint two of an ABB IRB 6620. The constant speed data are segmented around $\bar{\varphi}$ and the constant-speed friction levels are achieved based on (3). The procedure can be repeated for several $\overline{\dot{\varphi}}$ 's and a friction curve can be drawn, which contains steady-state friction values plotted against speed, see Figure $1 \mathrm{~b}$. The average time required to execute the trajectory to estimate friction at one speed was optimized down to $2.5 \mathrm{~s}$.

Friction data collected using such procedure simplifies the determination of wear related effects since the experiment is performed in a controlled manner, reducing the effects of external disturbances (found e.g. in contact applications) and it does not depend on a robot model, which may contain uncertainties. The fact that it does not account for possible direction dependencies of friction is not critical considering that wear would cause a generalized increase of friction which is captured by (3). Considering that performing experiments with the robot will reduce its availability, it is important to reduce the number of friction data required to provide accurate wear estimates. As it will be shown, the choice of which and how many speed levels where friction data are collected are important design parameters, affecting the quality of the wear estimates and the length of the experiments.

\subsection{A model for the nominal behavior of friction}

The behavior of friction in a robot joint is considerably affected by other variables than wear. To allow for a reliable discrimination of wear-related phenomena, it is therefore important that the effects caused by other variables are well understood. A common description of a direction independent friction curve is given according to

$$
\tau^{f}(\dot{\varphi})=f_{c}+f_{s} e^{-\left|\frac{\dot{\varphi}}{\varphi_{s}}\right|^{\alpha}}+f_{v} \dot{\varphi}
$$

which is valid for $\dot{\varphi}>0$ and where $f_{c}, f_{s}, f_{v}, \varphi_{s}$ and $\alpha$ are model parameters. The offset term $f_{c}$ is known as the Coulomb parameter; $f_{s} e^{-\left|\frac{\dot{\varphi}}{\varphi_{s}}\right|^{\alpha}}$ describes the decay of friction at intermediate speeds (Stribeck phenomenon) which is common in lubricated friction and tends to zero with speed according to the Stribeck speed parameter $\dot{\varphi}_{s}$ and exponent $\alpha$; the term $f_{v} \dot{\varphi}$ represents the viscous behavior of friction, increasing friction at high speeds, see Figure 1b. Based on a comprehensive experimental study of steady-state friction in an industrial robot joint, this model was extended in Bittencourt and Gunnarsson (2012) to include a descrip-

\footnotetext{
${ }^{\mathrm{i}}$ Throughout the paper all torques are normalized to the maximum manipulation torque at low speed and are therefore displayed as dimensionless quantities. All velocity measurements have values shown in the motor side, before the reduction.
} 
Table 1: Identified parameters for the model (5), values taken from Bittencourt and Gunnarsson (2012).

\begin{tabular}{cccccc}
\hline$f_{c, 0}$ & $f_{c, \ell}$ & $f_{s, 0}$ & $f_{s, \ell}$ & $f_{s, \xi}$ & $f_{v, 0}$ \\
$3.1110^{-2}$ & $2.3410^{-2}$ & $-2.5010^{-2}$ & $1.2610^{-1}$ & $1.6010^{-3}$ & $1.3010^{-4}$ \\
\hline$f_{v, \xi}$ & $\dot{\varphi}_{s, 0}$ & $\dot{\varphi}_{s, \ell}$ & $\dot{\varphi}_{s, \xi}$ & $\xi_{\mathrm{Vo}}$ & $\alpha$ \\
$1.3210^{-3}$ & -24.81 & 9.22 & 0.98 & 20.71 & 1.36 \\
\hline
\end{tabular}

tion of temperature and load according to

$$
\begin{aligned}
\tau_{0}^{f}\left(\dot{\varphi}, \tau^{\ell}, \xi\right) & =\left\{f_{c, 0}+f_{c, \ell} \tau^{\ell}\right\}+f_{s, \ell} \tau^{\ell} e^{-\left|\frac{\dot{\varphi}}{\dot{\varphi}_{s, \ell}}\right|^{\alpha}}+ \\
& \left\{f_{s, 0}+f_{s, \xi} \xi\right\} e^{-\left|\frac{\dot{\varphi}}{\left\{\dot{\varphi}_{s, 0}+\dot{\varphi}_{s, \xi} \xi\right\}}\right|^{\alpha}}+ \\
& \left\{f_{v, 0}+f_{v, \xi} e^{\frac{-\xi}{\xi}}\right\} \dot{\varphi},
\end{aligned}
$$

where $\tau^{\ell}$ is the absolute value of the manipulated load torque and $\xi$ is the joint temperature, the remaining variables are parameters used to model the friction behavior. The model (5) extends the parameters $f_{c}, f_{s}, \dot{\varphi}_{s}$ in (4) as a linear function of $\xi$ and $\tau^{\ell}$, where the exponential terms present a different behavior for $\tau^{\ell}$ and $\xi$; the viscous slope parameter $f_{v}$ is extended as a nonlinear function of $\xi$. A similar description of load has also been reported for different devices by Hamon et al. (2010); Kammerer and Garrec (2013). Marton and van der Linden (2012) also reported an exponential dependency of viscous friction with temperature.

In Bittencourt and Gunnarsson (2012), the parameters in (5) were found for joint two of an ABB IRB 6620 industrial robot with the use of joint temperature measurements and an estimate of $\tau^{\ell}$ based on a robot model; the parameter values are given in Table 1. Figure $2 \mathrm{a}$ presents observed and model-based predictions of friction curves for high and low values of $\tau^{\ell}$ and $\xi$. Notice the effects of $\tau^{\ell}$, which give an offset increase of the whole curve together with an exponentiallike increase at speeds below $25 \mathrm{rad} / \mathrm{s}$. The effects of $\xi$ can be seen as an exponential increase at speeds below $80 \mathrm{rad} / \mathrm{s}$ and a decrease of the curve slope at higher speeds. Notice further that for such temperature and load values, there is a speed range where the effects are less pronounced, in this case around $80 \mathrm{rad} / \mathrm{s}$.

\section{Validation}

As shown in Bittencourt and Gunnarsson (2012), the model in (5) can be used to predict the behavior of steady-state friction under broad operation conditions. This model can thus be used as a description of the nominal behavior of friction. The mean and standard deviation of the prediction error for the model in (5), denominated here as $\epsilon$, were estimated based on more than 5800 steady-state friction data points collected under different speed, temperature and load conditions as $\left[\mu_{\epsilon}, \sigma_{\epsilon}\right]=\left[-9.2410^{-4}, 4.2310^{-3}\right]$. The same evaluation for a model based on (4), dependent only on speed, gave a mean and standard deviation for the error 


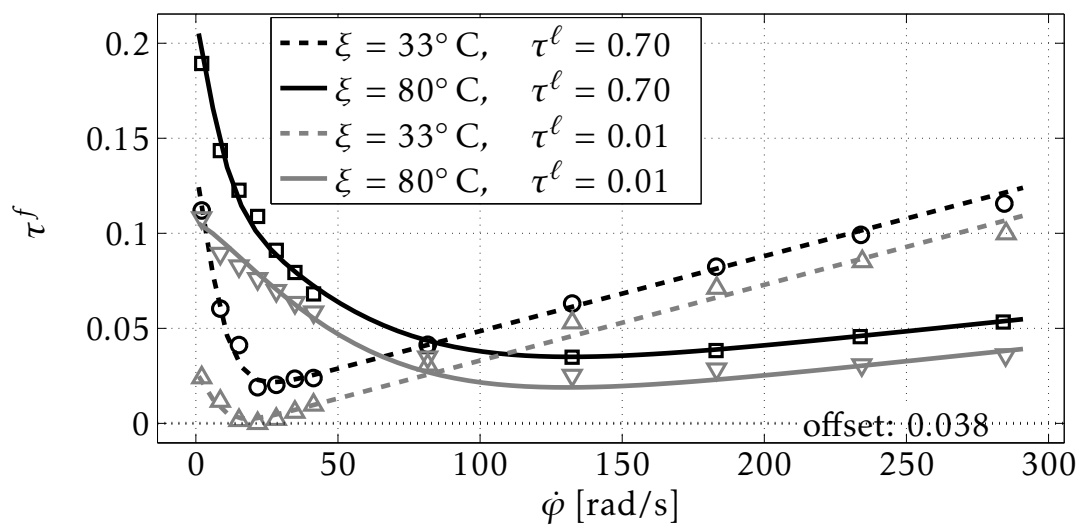

(a) Observed friction curves (markers) and model-based predictions (lines) given by (5) for low and high values of $\xi$ and $\tau^{\ell}$ and no significant wear.

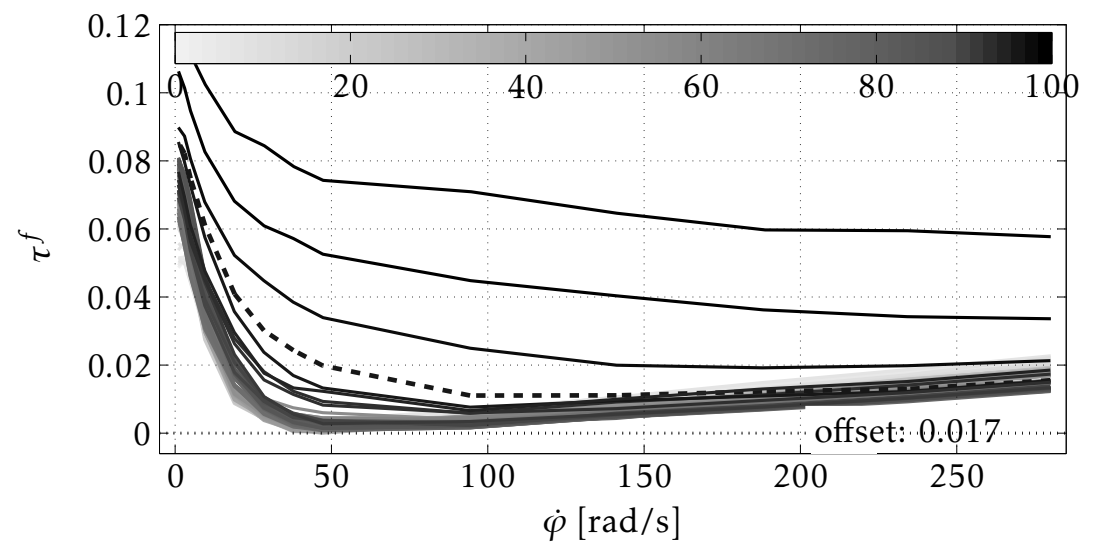

(b) Wear effects from accelerated tests. The colormap is related to the length of the tests with values between 0 and 100. The dashed line relates to a wear level critical for CBM.

Figure 2: Friction dependencies in a robot joint based on experimental studies. The offset values were removed for a comparison, their values are shown in the dotted lines. The data were collected for similar gearboxes and are presented in directly comparable scales. Notice the larger amplitude of effects caused by temperature and load compared to those caused by wear but the different speed dependencies. 
as $\left[1.0910^{-2}, 1.3410^{-2}\right]$ which are considerably larger.

\subsection{A model for the effects of wear to friction}

Monitoring a robot until a failure takes place is a costly and time consuming task and it is thus difficult to fully comprehend the effects of wear in a robot joint. An alternative is considered here based on data collected from accelerated wear tests, where the robot is run continuously under high load and stress levels for several months or years until failure. The resulting friction curves from such experiment at joint two of an ABB IRB 6620 robot are shown in Figure 2b, which were obtained under constant or nearly constant load-and temperature levels. The different speed dependencies of these effects compared to those caused by temperature and load in Figure $2 \mathrm{a}$ is an important characteristic of the problem. It shows that a careful selection of speed levels is needed to obtain an accurate determination of wear based on friction data.

Resolving for coupled effects between wear, temperature, load and other parameters would require costly long term experiments which are inviable even for accelerated tests. A simplifying assumption is taken that considers the effects of load and temperature to be additively separable from those caused by wear. Under this assumption, the effects of wear can be isolated in friction data collected under constant load and temperature conditions, such as the friction curves of Figure $2 \mathrm{~b}$. From such data, a wear profile quantity, $\widetilde{\tau}^{f}$, is defined by subtracting nominal friction data, observed before the accelerated wear tests started, $\tau_{0}^{f}$, from the ones obtained thereafter i.e.,

$$
\widetilde{\tau}^{f} \triangleq \tau^{f}-\tau_{0}^{f}
$$

The resulting wear profile from the accelerated wear tests in Figure $2 \mathrm{~b}$ can be seen in Figure 3, where friction is presented along speed $\dot{\varphi}$ and the experiment length $k$ with values between 0 and 100 .

As can be noticed, the effects of wear appear as an increase of friction in the low to intermediate speed region, and a small decrease of the viscous friction velocity slope. Introducing $\omega$ as a wear parameter, the observations support the choice of a model structure for the wear profile as

$$
\widetilde{\tau}^{f}(\dot{\varphi}, \omega)=f_{s, \omega} \omega e^{-\left|\frac{\dot{\varphi}}{\dot{\varphi}_{s, \omega} \omega}\right|^{\alpha}}+f_{v, \omega} \omega \dot{\varphi},
$$

where $f_{S, \omega}, \dot{\varphi}_{s, \omega}, f_{v, \omega}$ and $\alpha$ are model parameters. Except for the Coulomb term, the model has a similar structure as (4) with coefficients dependent on $\omega$. The variable $\omega$ relates to the degree of which the wear effects appear in the observed friction and it is not a physical quantity nor can it be measured. The wear parameter $\omega$ is defined by convention with values in the interval $(0,100)$, relative to a failure state, and is a dimensionless quantity.

The value $\omega=100$, denoting a failure state, should be defined as the point where the robot fails to perform according to the requirements imposed by the application. Clearly, a failure is always present in case the robot condition leads to a 


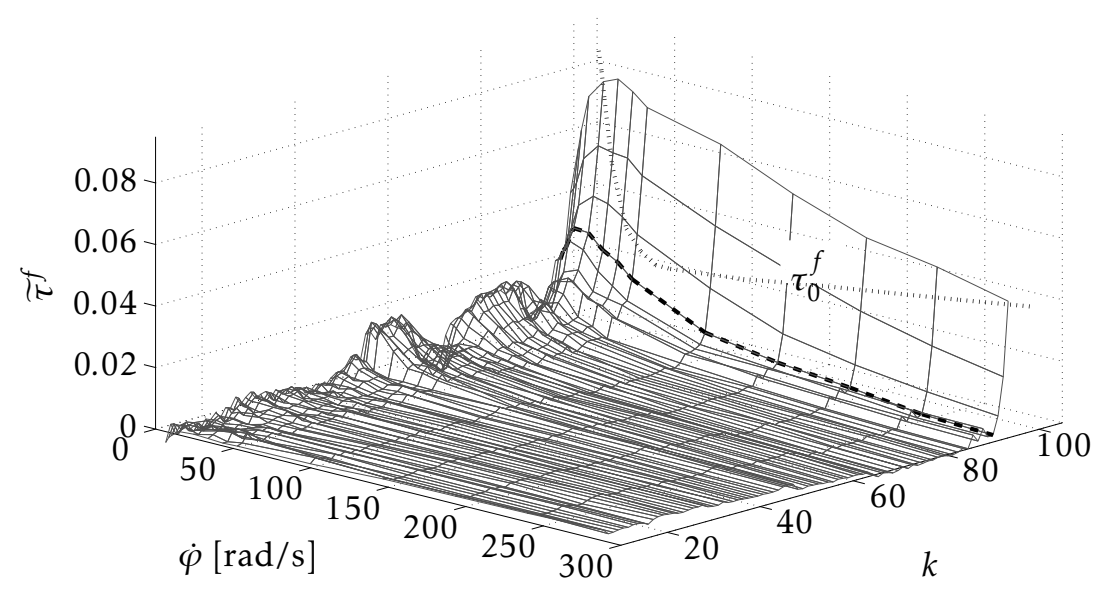

Figure 3: Friction wear profile $\widetilde{\tau}^{f}$ computed from the data in Figure $2 b a c$ cording to (6). The dotted line relates to the nominal friction curve $\tau_{0}^{f}$ removed from the friction data. The dashed line indicates a wear level considered important to be detected.

robot stop. While a robot stop may be caused by a total mechanical failure of the gearbox components, a more common situation is to have stops triggered by the safety supervision of the robot. The safety supervision may be triggered due to the presence of torque levels exceeding a maximum allowed level. This torque limit can thus be used to find the level of $\omega$ which would cause a robot stop by the safety supervision system.

In order to allow for condition based maintenance, the wear changes should be detected before a failure takes place. In fact, an alarm should be generated early enough so that appropriate maintenance actions can take place with minimal interference. Because wear will develop with time depending on how the robot is used, it is difficult to determinate a priori a critical wear level to be detected. This can be addressed with the development of lifetime models for prognosis which is outside the scope of this work. Often, lifetime models are developed based on the statistical behavior of failure data (Chick and Mendel, 1996) and are typically found during product development.

\section{Identification}

The model in (7) is identified with the wear profile data of Figure 3. For these data, a robot stop triggered by the safety supervision occurs at $k=100$ which is considered as a failure state. Based on a lifetime model developed for this robot, the robot manufacturer decided that in order to allow for CBM, it is critical to detect the wear level at $k=96.77$. Because it is important that the wear model is most accurate for this critical level, the data collected at $k=96.77$ are used 
Table 2: Parameters for the model in (7) and one standard deviation identified using the wear profile data at $k=96.77$ with wear fixed at $\omega=35$.

\begin{tabular}{ccc}
\hline$f_{s, \omega}\left[10^{-4}\right]$ & $f_{v, \omega}\left[10^{-7}\right]$ & $\dot{\varphi}_{s, \omega}$ \\
$9.02 \pm 0.19$ & $-5.15 \pm 1.00$ & $2.19 \pm 0.15$ \\
\hline
\end{tabular}

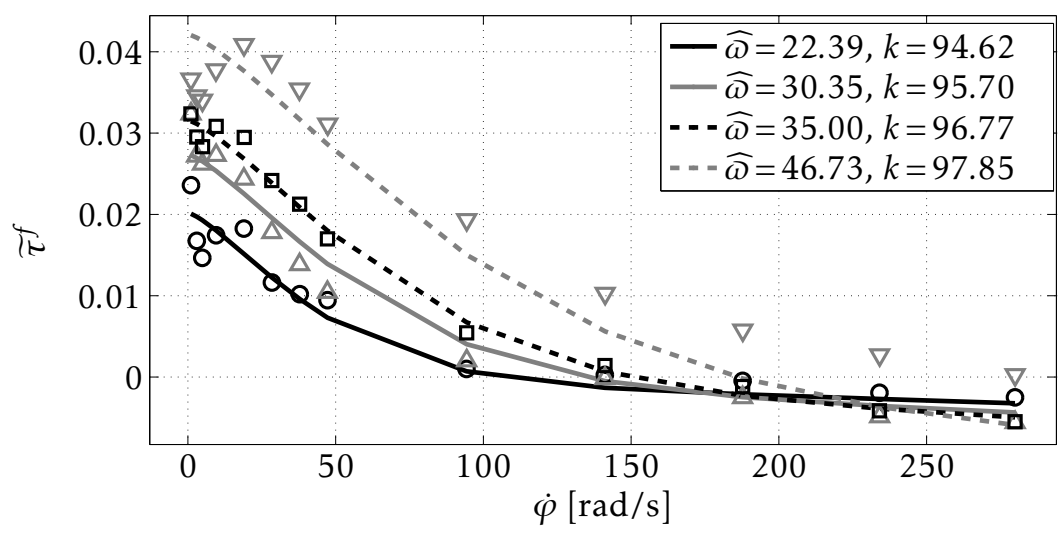

Figure 4: Observed wear profile data (circles) and model predictions (lines).

for the identification of the parameters of the wear model under the convention that $\omega=35$. This convention is adopted because the value of $\widetilde{\tau}^{f}$ at $k=96.77$ and $\dot{\varphi}=28 \mathrm{rad} / \mathrm{s}$ is around $35 \%$ of the maximum value of $\widetilde{\tau}^{f}$ for the entire data, which occurs at $k=100$ and same speed. The parameter $\alpha$ is fixed to 1.36 for consistency with the parameters found for (5), given in Table 1. The identification method described in Bittencourt and Gunnarsson (2012) is used to find the remaining parameters, which are shown in Table 2.

\section{Validation}

Considering the identified parameters for the model in (7), the wear levels of Figure 3 are identified for each $k$. With the identified wear values, the wear profile given by model predictions from (7) and observations are presented for the interval $k \in(94,98)$ in Figure 4 . As can be noticed, the model can predict well the behavior of $\widetilde{\tau}^{f}$. The estimated mean and standard deviation for the prediction error of the wear model in $(7)$, denoted here as $\widetilde{\epsilon}$, are $\left[\mu_{\widetilde{\epsilon}}, \sigma_{\widetilde{\epsilon}}\right]=\left[9.7210^{-4}, 3.8210^{-3}\right]$.

\subsection{A complete model of steady-state friction}

Under the assumption that the effects of load/temperature and wear are additively separable, it is possible to extend the model given in (5) to include the effects of wear as

$$
\tau^{f}\left(\dot{\varphi}, \tau^{\ell}, \xi, \omega\right)=\tau_{0}^{f}\left(\dot{\varphi}, \tau^{\ell}, \xi\right)+\widetilde{\tau}^{f}(\dot{\varphi}, \omega),
$$




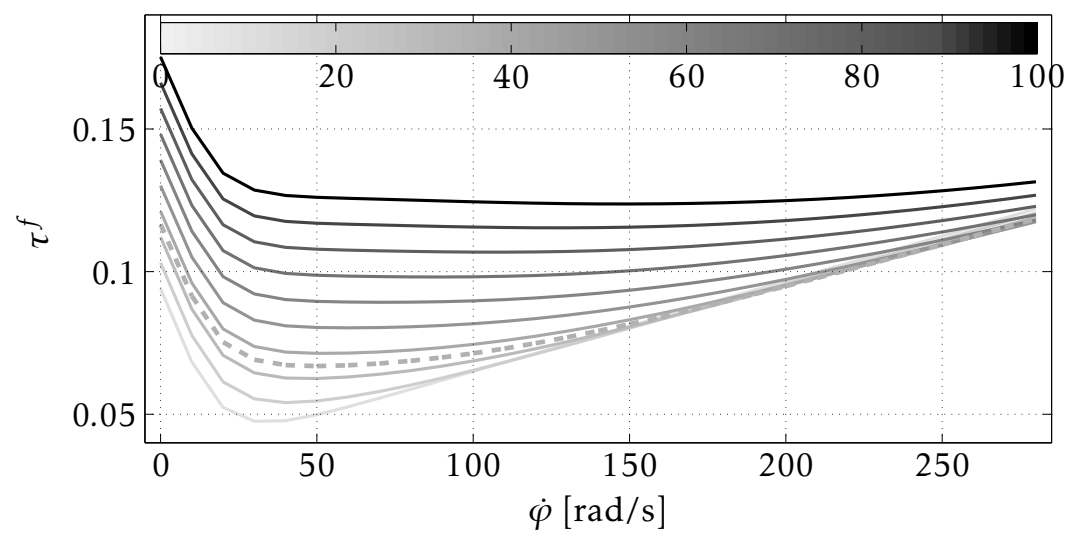

Figure 5: Friction curves for different wear levels given by the model (8) with temperature and load fixed at $\xi=40^{\circ} \mathrm{C}$ and $\tau^{\ell}=0.10$ respectively. The colormap indicates $\omega$ and the dashed line relates to the critical wear level $\omega=35$.

where $\tau_{0}^{f}\left(\dot{\varphi}, \tau^{\ell}, \xi\right)$ is given by (5) and $\widetilde{\tau}^{f}(\dot{\varphi}, \omega)$ is described in (7). Figure 5 presents the friction values given by the proposed model for $\xi=40^{\circ} \mathrm{C}$ and $\tau^{\ell}=$ 0.10 for wear values in the interval $\omega \in(0,100)$ when the parameters given in Tables 1 and 2 are used. Notice that the effects are appear first in the speed range between $0-150 \mathrm{rad} / \mathrm{s}$ and also that the resulting friction curves show good resemblance to Figure 2b. As previously, the dashed line in Figure 5 indicates an alarm level for the wear with $\omega=35$.

\section{Model-Based Wear Estimation}

Consider that the experiment described in Section 2.1 is repeated $N$ times independently at the speed levels

$$
\dot{\varphi}=\left[\begin{array}{llll}
\dot{\varphi}_{1}, & \dot{\varphi}_{2}, & \cdots, & \dot{\varphi}_{N}
\end{array}\right]^{T}
$$

resulting in the steady-state friction data points

$$
\tau^{f}=\left[\begin{array}{llll}
\tau_{1}^{f}, & \tau_{2}^{f}, & \cdots, & \tau_{N}^{f}
\end{array}\right]^{T} .
$$

To illustrate the situation where these experiments are performed at once, during the execution of a test-cycle, it is considered that the load, temperature and wear conditions are the same for these data. A model for each $i$ th steady-state friction datum $\tau_{i}^{f}$ can be achieved by including an additive uncertainty term to the model in (8). Assuming that the prediction errors for models (5) and (7) follow independent Gaussian distributions, $\epsilon \sim \mathcal{N}\left(\mu_{\epsilon}, \sigma_{\epsilon}^{2}\right)$ and $\widetilde{\epsilon} \sim \mathcal{N}\left(\mu_{\widetilde{\epsilon}}, \sigma_{\widetilde{\epsilon}}^{2}\right)$, the resulting data 
generation model is

$$
\tau_{i}^{f}=\tau_{0}^{f}\left(\dot{\varphi}_{i}, \tau^{\ell}, \xi\right)+\epsilon+\widetilde{\tau}^{f}\left(\dot{\varphi}_{i}, \omega\right)+\widetilde{\epsilon}=\tau^{f}\left(\dot{\varphi}_{i}, \tau^{\ell}, \xi, \omega\right)+\bar{\epsilon}
$$

where the resulting noise properties are given as

$$
\bar{\epsilon} \sim \mathcal{N}\left(\mu_{\bar{\epsilon}}, \sigma_{\bar{\epsilon}}^{2}\right), \quad \mu_{\bar{\epsilon}}=\mu_{\epsilon}+\mu_{\widetilde{\epsilon}}, \quad \sigma_{\bar{\epsilon}}^{2}=\sigma_{\epsilon}^{2}+\sigma_{\widetilde{\epsilon}}^{2} .
$$

Considering $\mu_{\bar{\epsilon}}^{-} \approx 0$, the joint density function for the friction data $\tau^{f}$ is

$$
p\left(\tau^{f} \mid \tau^{\ell}, \xi, \omega\right)=\mathcal{N}\left(\tau^{f} ; \tau^{f}\left(\dot{\varphi}, \tau^{\ell}, \xi, \omega\right), \Sigma\right)
$$

where $\Sigma=I \sigma_{\bar{\epsilon}}^{2}$ and

$$
\tau^{f}\left(\dot{\varphi}, \tau^{\ell}, \xi, \omega\right)=\left[\tau^{f}\left(\dot{\varphi}_{1}, \tau^{\ell}, \xi, \omega\right), \quad \tau^{f}\left(\dot{\varphi}_{2}, \tau^{\ell}, \xi, \omega\right), \quad \cdots, \quad \tau^{f}\left(\dot{\varphi}_{N}, \tau^{\ell}, \xi, \omega\right)\right]^{T} .
$$

An unbiased estimate of the load torque $\tau^{\ell}$ is considered available (e.g. achieved using a robot model) with distribution $\mathcal{N}\left(\tau^{\ell} ; \mu_{\ell}, \sigma_{\ell}^{2}\right)$. The information from this estimate is included in the model by considering the marginal density function

$$
\bar{p}\left(\tau^{f} \mid \xi, \omega\right)=\int_{-\infty}^{\infty} p\left(\tau^{f} \mid \tau^{\ell}, \xi, \omega\right) \mathcal{N}\left(\tau^{\ell} ; \mu_{\ell}, \sigma_{\ell}^{2}\right) \mathrm{d} \tau^{\ell}
$$

which for the Gaussian distribution $p\left(\tau^{f} \mid \tau^{\ell}, \xi, \omega\right)$ given in (10) can be found explicitly since the dependence of the mean $\tau^{f}\left(\dot{\varphi}, \tau^{\ell}, \xi, \omega\right)$ is linear on $\tau^{\ell}$, recall (5). The marginal density function is given by (Bishop, 2006, p. 93)

$$
\bar{p}\left(\tau^{f} \mid \xi, \omega\right)=\mathcal{N}\left(\tau^{f} ; \tau^{f}(\dot{\varphi}, \xi, \omega), \Sigma(\dot{\varphi})\right)
$$

where

$$
\begin{aligned}
\tau^{f}(\dot{\varphi}, \xi, \omega) & \triangleq \tau^{f}\left(\dot{\varphi}, \mu_{\ell}, \xi, \omega\right) \\
\Sigma(\dot{\varphi}) & =\Sigma+s(\dot{\varphi}) s(\dot{\varphi})^{T} \sigma_{\ell}^{2} \\
s(\dot{\varphi}) & \triangleq\left[s\left(\dot{\varphi}_{1}\right), \quad s\left(\dot{\varphi}_{2}\right), \quad \cdots, s\left(\dot{\varphi}_{N}\right)\right]^{T} \\
s(\dot{\varphi}) & \triangleq f_{c, \ell}+f_{s, \ell} e^{-\left|\frac{\dot{\varphi}}{\dot{\varphi}_{s, \ell}}\right|^{\alpha}} .
\end{aligned}
$$

It is further considered that the model parameters are known. In this setting, the vector of unknowns is $\theta=[\xi, \Theta]^{T}$ and has the log-likelihood function

$$
\log \mathcal{L}(\theta)=\log \mathcal{N}\left(\tau^{f} ; \tau^{f}(\dot{\varphi}, \theta), \Sigma(\dot{\varphi})\right) .
$$

Based on the achieved likelihood function, Section 3.1 discusses maximum likelihood estimators of $\omega$. The estimate is dependent on the data $\tau^{f}$ and thus on the choice of speed levels $\dot{\varphi}$. For a limited number of friction observations $N$, the problem of experiment design is to choose $\dot{\varphi}$ such that the estimated wear level is as accurate as possible. Experiment design is described in Section 3.2. 


\subsection{Maximum likelihood estimation}

The maximum likelihood estimate of $\theta$ given the data vector $\tau^{f}$ is the value for which the log-likelihood function, given in (15), has a maximum, i.e.

$$
\widehat{\boldsymbol{\theta}}=\arg \max _{\boldsymbol{\theta}} \log \mathcal{L}(\boldsymbol{\theta}) \text {. }
$$

The terms dependent on $\theta$ in the $\log$-likelihood function have the form

$$
\log \mathcal{L}(\boldsymbol{\theta}) \propto-\left[\boldsymbol{\tau}^{f}-\boldsymbol{\tau}^{f}(\dot{\boldsymbol{\varphi}}, \boldsymbol{\theta})\right]^{T} \sum(\dot{\boldsymbol{\varphi}})^{-1}\left[\boldsymbol{\tau}^{f}-\tau^{f}(\dot{\boldsymbol{\varphi}}, \boldsymbol{\theta})\right],
$$

and the problem is therefore a weighted nonlinear least-squares, where $\xi$ and $\omega$ are estimated jointly. To restrict the search space, it is possible to add constraints to the problem according to available knowledge of the unknowns. Naturally, $\omega \geq 0$, and it is also possible to include lower and upper limits for the temperature, denoted $\underline{\xi}$ and $\bar{\xi}$ respectively. For a robot operating in a controlled indoor environment, $\underline{\xi}$ would be the minimum room temperature while $\bar{\xi}$ is given by the maximum room temperature and self heating of the joint due to actuator losses. This gives the problem

$$
\begin{aligned}
& {[\widehat{\xi}, \widehat{\omega}]=\arg \min _{\xi, \omega}\left[\tau^{f}-\tau^{f}(\dot{\varphi}, \xi, \omega)\right]^{T} \sum(\dot{\varphi})^{-1}\left[\tau^{f}-\tau^{f}(\dot{\varphi}, \xi, \omega)\right]} \\
& \text { s.t. } \quad 0 \leq \omega \\
& \quad \underline{\xi} \leq \xi \leq \bar{\xi},
\end{aligned}
$$

which is solved using lsqnonlin available in Matlab's Optimization Toolbox with initial values found from a coarse grid search.

The estimator in (16) is valid for $N \geq 2$ since at least two equations are needed to solve for the two unknowns. For $N=1$, the effects of temperature can be marginalized away. Considering that temperature $\xi$ can occur with equal probability over its domain, i.e. $\xi \sim \mathcal{U}(\underline{\xi}, \bar{\xi})$, the marginalized likelihood function is,

$$
\bar{p}\left(\tau^{f} \mid \omega\right)=\frac{1}{\bar{\xi}-\underline{\xi}} \int_{\underline{\xi}}^{\bar{\xi}} \bar{p}\left(\tau^{f} \mid \xi, \omega\right) \mathrm{d} \xi .
$$

Since there is no analytical solution for (17), Monte Carlo Integration (MCI) is used to approximate it in a symbolic expression in $\omega$ as

$$
\widehat{p}\left(\boldsymbol{\tau}^{f} \mid \omega\right)=\frac{1}{N_{\xi}} \sum_{i=1}^{N_{\xi}} \bar{p}\left(\boldsymbol{\tau}^{f} \mid, \xi^{(i)}, \omega\right)
$$

for $N_{\xi}$ randomly generated samples $\xi^{(i)} \sim \mathcal{U}(\underline{\xi}, \bar{\xi})$.

Using the approximated marginalized likelihood function of (18) leads to the 
problem

$$
\begin{gathered}
\widehat{\omega}=\arg \max _{\omega} \log \widehat{p}\left(\tau^{f} \mid \omega\right) \\
\text { s.t. } \quad 0 \leq \omega .
\end{gathered}
$$

This nonlinear constrained minimization is solved using fmincon from the Optimization Toolbox in Matlab with initial values taken from a coarse grid search.

\subsection{Experiment design}

An estimate $\widehat{\theta}$ of $\theta$ is dependent on the data $\tau^{f}$, the associated $\dot{\varphi}$ and on the estimator used. The mean square error (MSE) of an estimate can be used as a criterion to assess how the choice of $\dot{\varphi}$ affects the performance. Let the bias of an estimate $\widehat{\boldsymbol{\theta}}$ be denoted $\boldsymbol{b}(\widehat{\boldsymbol{\theta}}) \triangleq \mathbb{E}[\widehat{\boldsymbol{\theta}}]-\boldsymbol{\theta}^{\prime}$ then

$$
\operatorname{MSE}(\widehat{\boldsymbol{\theta}})=\mathbb{E}\left[\left(\widehat{\boldsymbol{\theta}}-\boldsymbol{\theta}^{\prime}\right)^{T}\left(\widehat{\boldsymbol{\theta}}-\boldsymbol{\theta}^{\prime}\right)\right]=\operatorname{tr}(\operatorname{Cov}(\widehat{\boldsymbol{\theta}}))+\boldsymbol{b}(\widehat{\boldsymbol{\theta}})^{T} \boldsymbol{b}(\widehat{\boldsymbol{\theta}}) .
$$

Considering unbiased estimators, the Cramér-Rao lower bound for unbiased estimators gives

$$
\operatorname{MSE}(\widehat{\boldsymbol{\theta}}) \geq \operatorname{tr}\left(\mathcal{F}\left(\boldsymbol{\theta}^{\prime}\right)^{-1}\right)
$$

where

$$
\mathcal{F}\left(\boldsymbol{\theta}^{\prime}\right) \triangleq \mathbb{E}\left[\left.\left(\frac{\partial \log \mathcal{L}(\boldsymbol{\theta})}{\partial \boldsymbol{\theta}}\right)\left(\frac{\partial \log \mathcal{L}(\boldsymbol{\theta})}{\partial \boldsymbol{\theta}}\right)^{T}\right|_{\boldsymbol{\theta}=\boldsymbol{\theta}^{\prime}}\right]
$$

is the Fisher information matrix. The achieved bound can be minimized by affecting the inverse of the Fisher information matrix, improving the achievable performance for any unbiased estimator. For the log-likelihood function in (15), the Fisher information matrix is given by (Porat and Friedlander, 1986)

$$
\mathcal{F}\left(\dot{\varphi}, \theta^{\prime}\right)=\left.\left(\frac{\partial \tau^{f}(\dot{\varphi}, \theta)}{\partial \theta}\right)^{T} \Sigma(\dot{\varphi})^{-1}\left(\frac{\partial \tau^{f}(\dot{\varphi}, \theta)}{\partial \theta}\right)\right|_{\boldsymbol{\theta}=\boldsymbol{\theta}^{\prime}}
$$

where the dependence on $\dot{\varphi}$ is highlighted.

The objective of the experiment is to determine the wear level $\omega$ as accurately as possible. This can be achieved by an appropriate choice of the speed levels $\dot{\varphi}$, which are the design variables. For $\theta=[\xi, \omega]^{T}$, the lower bound on $\operatorname{MSE}(\widehat{\omega})$ corresponds to the 2,2-element of the inverse of the information matrix given by (23). Using this bound as a criterion for experiment design gives the problem

$$
\dot{\varphi}^{*}=\arg \min _{\dot{\varphi}}\left[\mathcal{F}\left(\dot{\varphi}, \theta^{\prime}\right)^{-1}\right]_{2,2},
$$

where $[\cdot]_{i, j}$ denotes the $i, j$-element of a matrix. Dropping the arguments for $\mathcal{F}\left(\dot{\varphi}, \theta^{\prime}\right)$, the analytical expression for $\left[\mathcal{F}^{-1}\right]_{2,2}$ is given by

$$
\left[\mathcal{F}^{-1}\right]_{2,2}=\frac{[\mathcal{F}]_{1,1}}{[\mathcal{F}]_{1,1}[\mathcal{F}]_{2,2}-[\mathcal{F}]_{1,2}^{2}}
$$


For a positive definite $\Sigma(\dot{\varphi})$, the problem is well-posed only if $\frac{\partial \boldsymbol{\tau}^{f}(\dot{\boldsymbol{\varphi}}, \boldsymbol{\theta})}{\partial \boldsymbol{\theta}}$ has rank equal to the number of unknowns, i.e. two. This can only be achieved for $N \geq 2$ and if there are at least two linear independent columns in $\frac{\partial \tau^{f}(\dot{\varphi}, \theta)}{\partial \theta}$. A necessary condition for the latter is that at least two of the speed levels chosen are different, sufficiency will depend on the specific choice of friction model and value of $\theta^{\prime}$. To ensure that no same speed is chosen, additional constraints are included in (24) to require a minimum separation, $\delta_{\dot{\varphi}}$, between speed levels in $\dot{\varphi}$. Furthermore, the search is limited to the minimum, $\dot{\varphi}$, and maximum, $\overline{\dot{\varphi}}$, speed levels for which the experiment of Section 2.1 can be performed. The optimal speed values are therefore given as the solution to the problem

$$
\begin{array}{ll}
\dot{\varphi}^{*}= & \arg \min _{\dot{\varphi}}\left[\mathcal{F}\left(\dot{\varphi}, \theta^{\prime}\right)^{-1}\right]_{2,2} \\
\text { s.t. } & \dot{\varphi}_{i}-\dot{\varphi}_{j} \leq-\delta_{\dot{\varphi}}, \quad(i<j) \\
& \underline{\dot{\varphi}} \leq \dot{\varphi}_{i} \leq \overline{\dot{\varphi}}
\end{array}
$$

This is a constrained nonlinear minimization which is solved here using fmincon in Matlab with initial values found from a coarse grid search.

The case where $N=1$ can be considered by using the approximated marginalized likelihood function given by (18). Using this approximation, the Fisher information matrix is

$$
\mathcal{F}\left(\dot{\varphi}, \omega^{\prime}\right) \triangleq \mathbb{E}\left[\left.\left(\frac{\partial \log \widehat{p}\left(\tau^{f} \mid \omega\right)}{\partial \omega}\right)^{2}\right|_{\omega=\omega^{\prime}}\right] .
$$

The differentiation of $\widehat{p}(\cdot)$ is performed symbolically and the expectation is computed using MCI with $N_{f}$ samples taken from $\widehat{p}\left(\tau^{f} \mid \omega\right)$ in (18), leading to the estimate $\widehat{\mathcal{F}}\left(\dot{\varphi}, \omega^{\prime}\right)$ of $\mathcal{F}\left(\dot{\varphi}, \omega^{\prime}\right)$. The associated optimization problem is thus

$$
\begin{aligned}
& \dot{\varphi}^{*}=\underset{\dot{\varphi}}{\arg \min _{\dot{\varphi}} \widehat{\mathcal{F}}\left(\dot{\varphi}, \omega^{\prime}\right)^{-1}} \\
& \text { s.t. } \quad \dot{\varphi} \leq \dot{\varphi}_{i} \leq \overline{\dot{\varphi}}
\end{aligned}
$$

which is also a constrained nonlinear minimization problem and is solved in the same manner as (26).

\section{Simulation Study}

A simulation study is first considered to illustrate the use of the experiment design criteria defined in Section 3.2 and wear estimators proposed in Section 3.1.

\subsection{Definition of parameters used}

The framework of Section 3 requires knowledge of the friction model parameters in the data generation model (9). The parameters for the nominal part given 
Table 3: Optimization parameters.

\begin{tabular}{ccccccc}
\multicolumn{2}{c}{ Experiment design } & \multicolumn{2}{c}{ Identification } & \multicolumn{2}{c}{ Approximations } \\
\hline$\dot{\varphi}$ & $\overline{\dot{\varphi}}$ & $\delta_{\dot{\varphi}}$ & $\underline{\xi}$ & $\bar{\xi}$ & $N_{\xi}$ & $N_{f}$ \\
$\overline{1}$ & 280 & 5 & 30 & 50 & 100 & 200 \\
\hline
\end{tabular}

Table 4: Choice of optimal speed values for different values of $N$. "Cost" is the value of the objective function in $(27)(N=1)$ or $(26)(N \geq 2)$ computed at $\dot{\varphi}^{*}$.

\begin{tabular}{ccc}
$N$ & Cost & $\dot{\varphi}^{*}$ \\
\hline 1 & 45.91 & 33.78 \\
2 & 26.01 & {$[35.84,40.84]^{T}$} \\
3 & 19.65 & {$[33.68,38.68,43.68]^{T}$} \\
4 & 16.50 & {$[31.65,36.65,41.65,46.65]^{T}$} \\
\hline
\end{tabular}

in (5) can be identified for a new robot using joint temperature measurements and an estimate of the joint load torques, see e.g. Bittencourt and Gunnarsson (2012). The parameters for (7), describing the wear behavior, are more difficult because failure data are required. For CBM, wear estimates are needed before a failure of the system, in which case the parameters for (7) cannot be known in advance. This can be overcome with the use of historical failure data. The simulation studies that follows illustrate the case where these model parameters are known, focusing on the effects of temperature and load uncertainties. In Section 5, the effects of uncertainties in the wear model are studied based on real data.

Here, the friction parameters used are given in Tables 1 and 2 which were identified for joint two of an ABB IRB 6620 industrial robot. The noise properties of (9b) are taken from the model validation in Sections 2.2 and 2.3. Applying (9b) to these values gives $\mu_{\bar{\epsilon}}^{-}=4.8010^{-5} \approx 0$ and $\sigma_{\bar{\epsilon}}^{-}=5.7010^{-3}$. The mean and standard deviation for the load estimate used in (11) are chosen as $\mu_{\ell}=0.5$ and $\sigma_{\ell}=0.1$. Finally, the optimization parameters used in the identification and experiment design problems are given in Table 3.

\subsection{Experiment design}

As discussed in Section 3, the objective of experiment design is to choose $\dot{\varphi}$ that gives as high accuracy as possible for the wear estimate. From a practical perspective, it is also important to limit the number of friction data points $N$. Here, the experiment design will be considered for $N \in\{1,2,3,4\}$. For friction data collected according to the experiment defined in Section 2.1, this would give up to one minute of total experimentation time for a six axis robot. The problems (27) and (26) are solved for $N=1$ and $N \in\{2,3,4\}$ respectively when temperature and wear are fixed to $\xi=40^{\circ} \mathrm{C}$ and $\omega=35$. The optimal speed values found are shown in Table 4 , which have values in the interval $(30,50) \mathrm{rad} / \mathrm{s}$. 


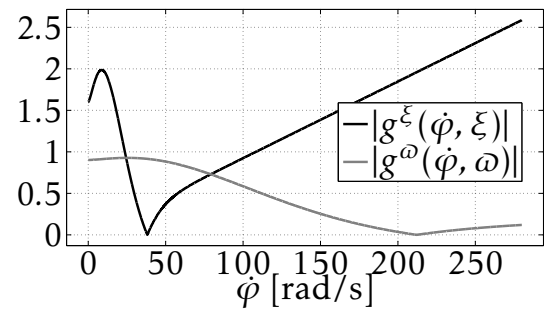

(a) Absolute value of the derivatives $\left|g^{\xi}(\dot{\varphi}, \xi)\right|$ and $\left|g^{\Theta}(\dot{\varphi}, \omega)\right|$.

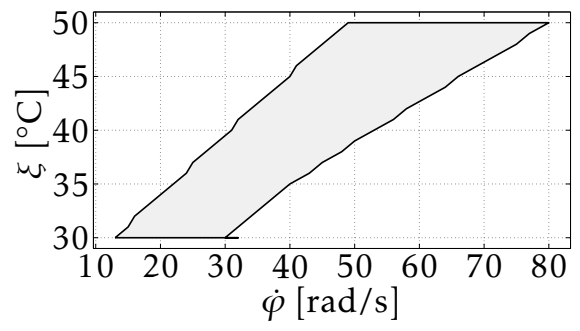

(b) Speed region where $\left|g^{\oplus}(\dot{\varphi}, \omega)\right|>$ $2\left|g^{\xi}(\dot{\varphi}, \xi)\right|$.

Figure 6: (a) Behavior of $g^{\omega}(\dot{\varphi}, \omega)$ and $g^{\xi}(\dot{\varphi}, \xi)$ with respect to speed evaluated at $\xi=40^{\circ} \mathrm{C}$ and $\omega=35$. (b) The speed regions which give $\left|g^{\omega}(\dot{\varphi}, \omega)\right|>$ $2\left|g^{\xi}(\dot{\varphi}, \xi)\right|$ when $\omega=35$ and $\xi \in(30,50)^{\circ} \mathrm{C}$.

To provide more insights on the experiment design problem, note that the information matrix used in the optimal solution for (26) is dependent on products of the derivatives

$$
\left.g^{\xi}\left(\dot{\varphi}, \xi^{\prime}\right) \triangleq \frac{\partial \tau^{f}(\dot{\varphi}, \xi, \omega)}{\partial \xi}\right|_{\xi=\xi^{\prime}},\left.\quad g^{\omega}\left(\dot{\varphi}, \omega^{\prime}\right) \triangleq \frac{\partial \tau^{f}(\dot{\varphi}, \xi, \omega)}{\partial \omega}\right|_{\omega=\omega^{\prime}}
$$

which, because of the model structure, recall (13) and (8), are function only of $\dot{\varphi}$ and the differentiation variable. These derivatives relate to the information about temperature, $\xi$, and wear, $\omega$, contained in the model. For instance, if $\Sigma(\dot{\varphi})=I$, the objective function $\left[\mathcal{F}^{-1}\right]_{2,2}$ in $(25)$ is

$$
\frac{\sum_{i=1}^{N} g^{\xi}\left(\dot{\varphi}_{i}, \xi\right)^{2}}{\left(\sum_{i=1}^{N} g^{\xi}\left(\dot{\varphi}_{i}, \xi\right)^{2}\right)\left(\sum_{i=1}^{N} g^{\omega}\left(\dot{\varphi}_{i}, \omega\right)^{2}\right)-\left(\sum_{i=1}^{N} g^{\xi}\left(\dot{\varphi}_{i}, \xi\right) g^{\omega}\left(\dot{\varphi}_{i}, \omega\right)\right)^{2}} .
$$

The objective of the experiment design is to gather information about $\omega$, i.e. minimize $\left[\mathcal{F}^{-1}\right]_{2,2}$, and it is hence natural that speed points are selected where $\left|g^{\omega}(\dot{\varphi}, \omega)\right|$ is larger than $\left|g^{\xi}(\dot{\varphi}, \xi)\right|$. These gradients are shown in Figure 6a as a function of speed $\dot{\varphi}$ for the fixed $\xi=40^{\circ} \mathrm{C}$ and $\omega=35$. For speed levels in the interval $(30,50) \mathrm{rad} / \mathrm{s}$, it is possible to note that $\left|g^{\omega}(\dot{\varphi}, \omega)\right|$ is always at least twice as large as $\left|g^{\xi}(\dot{\varphi}, \xi)\right|$.

The model derivatives (28) are dependent on the operating point for $\xi$ and $\omega$ and it is not possible to select $\dot{\varphi}$ that is optimal globally. To illustrate these dependencies, Figure 7 shows contour plots of $\left|g^{\xi}(\dot{\varphi}, \xi)\right|$ and $\left|g^{\omega}(\dot{\varphi}, \omega)\right|$ for a wide range of speed, temperature and wear values. The dashed lines in Figure 7 relate to the value where the derivatives are zero. In both sub figures, the derivatives have negative values to the right of the dashed lines and are otherwise positive. The 

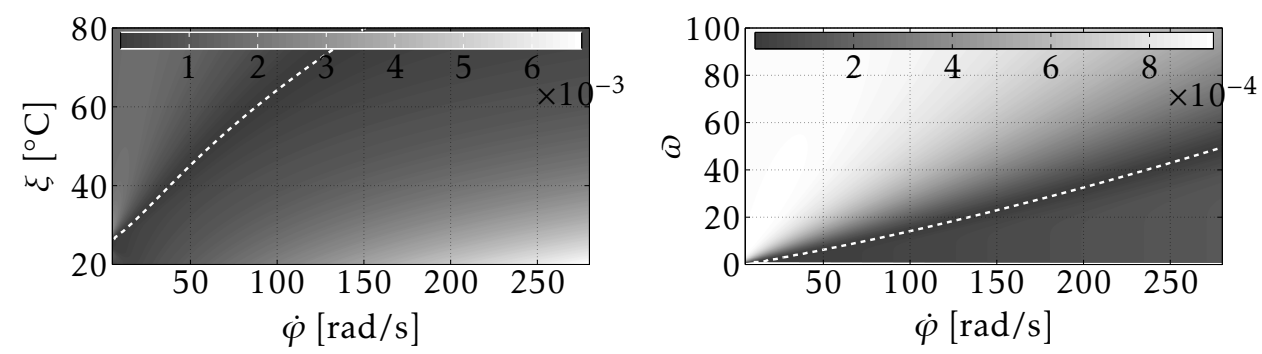

Figure 7: Contours of $\left|g^{\xi}(\dot{\varphi}, \xi)\right|$ (left) and $\left|g^{\Theta}(\dot{\varphi}, \omega)\right|$ (right) as a function of speed and the differentiation variable. Notice the difference in the scales.

derivative for temperature is often larger than that for wear. Nevertheless, the different speed dependencies allow for a selective choice of $\dot{\varphi}$ to improve performance of the wear estimates.

To illustrate how the optimal speed region can vary with operating points, Figure $6 \mathrm{~b}$ displays the speed region where $\left|g^{\omega}(\dot{\varphi}, \omega)\right|>2\left|g^{\xi}(\dot{\varphi}, \xi)\right|$ when $\omega=35$, i.e. the critical value to be detected, and $\xi \in(30,50)^{\circ} \mathrm{C}$. Notice that this speed region is not optimal in the sense of (26) or (27), but relates to a region where the information for $\omega$ is considerably larger than for $\xi$. As it can be seen, only a narrow band of speed values contain useful information for the estimation of $\omega$. The speed band also varies with temperature, with no overlap over all temperature values considered.

It is important to emphasize that the characteristics shown here are valid for the specific model parameters used and different properties are expected for different robots and gearboxes. Nevertheless, a similar behavior of temperature and wear has been observed for various robot units equipped with a similar type of gearbox.

\subsection{Bias and variance properties of the wear estimators}

With the optimal speed values found in Table 4, the bias and variance properties of the proposed estimators are assessed based on Monte Carlo simulations. The true wear level is fixed at $\omega=35$ and temperature is varied in the interval $\xi \in$ $(30,50)^{\circ} \mathrm{C}$. The data generated by (9) are input to (19) or (16) for $N=1$ and $N \in$ $\{2,3,4\}$ respectively and the estimation is repeated a total of $N_{\mathrm{MC}}=110^{3}$ times per operating point.

Figure 8 shows the simulation results for the bias and variance of the estimators as a function of the temperature level $\xi$. As it can be seen, the bias and variance are reduced with $N$. The reduction in the variance is specially large for $N=2$ compared to $N=1$, which is related to marginalization effects of $\xi$. The bias presents a nonlinear behavior with $\xi$ while the variance seems unaffected by it. 

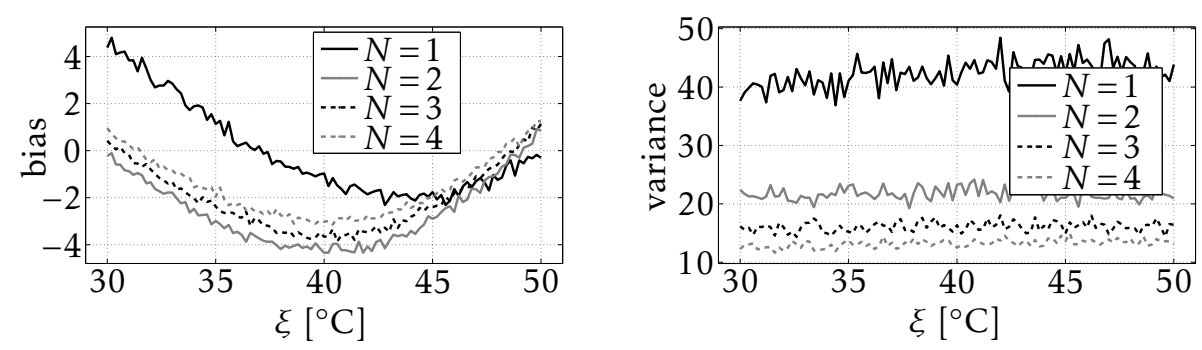

Figure 8: Monte Carlo based estimates of the bias (left) and the variance (right) for the wear estimators (19) and (16) $(N=1$ and $N \in\{2,3,4\}$ respectively) evaluated for $\omega=35$ and $\xi \in(30,50)^{\circ} \mathrm{C}$.

\section{Studies based on Real Data}

Gathering enough informative data related to wear from the field would have been inviable since wear faults take a long time to develop and are infrequent. Even in accelerated wear tests, it may take several months or years before wear effects become significant. Another difficulty with such tests is the high cost of running several robots to obtain reliable statistics. Temperature studies are also challenging since the thermal constant of a large robot is of several hours.

Other than simulation studies, the only viable alternative in the research project was to combine nominal friction data (with no acute wear present) and wear profile data collected from a different robot of the same type. These data were collected from axis two of ABB IRB 6620 industrial robots equipped with rotary vector gearboxes. Each of these data sets are matrices where each row contains data from a friction curve collected in the following velocity values

$$
\begin{array}{r}
\dot{\varphi}=[2.1,8.7,15.3,21.9,28.5,35.1,41.7, \\
82.2,133.5,184.7,236.2,287.1],
\end{array}
$$

i.e., a total of twelve different speed values are possible from these data sets. The nominal friction data matrix, $F^{0}$, has rows associated to different load and temperature conditions which are stored in matrices of the same size as $F^{0}$ denoted $L$ and $T$ respectively. The wear profile data matrix, $\widetilde{F}$, contains data collected from accelerated wear tests under constant load and temperature conditions and has rows associated to the experimentation index $k$.

The wear profile data set determines the behavior of friction as a function of $k$. For a given wear profile data set, the objective is to emulate friction data collected under varying conditions of load and temperature. First, the desired load and temperature behaviors are pre-defined as a function of $k$ according to $\xi(k) \in T$ and $\tau^{\ell}(k) \in L$. Second, the data sets are combined as a function of $k$ and the 
desired speed $\dot{\varphi} \in \dot{\varphi}$ to provide friction data according to

$$
\begin{aligned}
\tau^{f}(k, \dot{\varphi}) & =\left[F^{0}\right]_{i_{k}, j_{\dot{\varphi}}}+[\widetilde{F}]_{k, j_{\dot{\varphi}}} \\
j_{\dot{\varphi}} & \triangleq\left\{j:[\dot{\varphi}]_{j}=\dot{\varphi}\right\}, \\
i_{k} & \triangleq\left\{i:[T]_{i, j_{\dot{\varphi}}}=\xi(k) \text { and }[L]_{i, j_{\dot{\varphi}}}=\tau^{\ell}(k)\right\} .
\end{aligned}
$$

Notice that these data are not analytically generated, but actually based on friction data collected with the experiment described in Section 2.1. Furthermore, the combination of data according to (30a) is consistent to the model structure in (8) and with the assumption that the effects of load/temperature and wear are additively separable.

\subsection{Description of scenarios}

Three different wear profile data sets are considered, they are assigned as $\widetilde{F}^{0}, \widetilde{F}^{1}$ and $\widetilde{F}^{2}$. The data set $\widetilde{F}^{0}$ was used for the wear modeling presented in Section 2.3, and is shown in Figure 3 and the other two are shown in Figure 9. Some relevant characteristics of the wear profile data sets are listed next where the quantity

$$
r_{\max }(F, G) \triangleq \frac{\max _{i j}\left|F_{i j}\right|}{\max _{i j}\left|G_{i j}\right|}
$$

denotes the ratio of maximum absolute values of the matrices $F$ and $G$ and is used for a comparison.

$\widetilde{F}^{0}$ Presents small random variations, remaining around 0 up to $k=90$ followed by an exponential increase thereafter.

$\widetilde{F}^{1}$ Presents medium random variations, remaining around 0 up to $k=70$ followed by large increases. Relative to $\widetilde{F}^{0}$ it gives $r_{\max }\left(\widetilde{F}^{1}, \widetilde{F}^{0}\right)=56 \%$.

$\widetilde{F}^{2}$ Presents small random variations, remaining stationary up to $k=30$ followed by small increases up to $k=97$ from where it increases steeply. Relative to $\widetilde{F}^{0}$ it gives $r_{\max }\left(\widetilde{F}^{2}, \widetilde{F}^{0}\right)=106 \%$.

Three scenarios are considered in the study based on the data set pairs $\left(F^{0}, \widetilde{F}^{0}\right)$, $\left(F^{0}, \widetilde{F}^{1}\right)$ and $\left(F^{0}, \widetilde{F}^{2}\right)$. The scenarios are denoted 0,1 or 2 according to the selected data set for the wear profile. The same model and optimization parameters considered in Section 4.1 are used for all scenarios. The parameters for the friction model were identified based on the data sets $F^{0}$ and $\widetilde{F}^{0}$. Since these parameters are used for all scenarios, it can be considered that the parameters for the nominal behavior of friction are consistent for all scenarios. This illustrates the situation where the nominal friction parameters are found based on experiments performed when the joint is healthy. The wear-related parameters are only consistent for Scenario 0 and the other scenarios illustrate the situation where the wear parameters are based on historical failure data. 
Table 5: Choice of optimal speed values for different values of friction observations $N$.

\begin{tabular}{ccc}
$N$ & Cost & $\dot{\varphi}^{*}$ \\
\hline 1 & 46.58 & 35.1 \\
2 & 26.20 & {$[35.1,41.7]^{T}$} \\
3 & 22.60 & {$[28.5,41.7,82.2]^{T}$} \\
4 & 18.00 & {$[2.1,28.5,35.1,41.7]^{T}$} \\
\hline
\end{tabular}

To simplify the presentation of the results, the behavior of $\xi(k)$ and $\tau^{\ell}(k)$ are the same for the three scenarios, they are shown in Figure 10. Notice that the amplitude of the friction changes due to temperature and load are considerably larger than of those caused by wear for any of the scenarios.

\subsection{Results and discussion}

The choice of speed values for experiment design is limited to the speed levels available from the data sets as given in (29). The problems (26) and (27) are solved by considering every possible combination of speed levels for $N \in$ $\{1,2,3,4\}$. The resulting optimal values are given in Table 5 and relate well to those found in Table 4 . Notice that the optimal speed values depend on the wear model parameters used. Since these parameters were found using $\widetilde{F}^{0}$, optimality of $\dot{\varphi}^{*}$ is only expected for Scenario 0 .

The resulting wear estimates for the different scenarios are shown in Figures 11a to 11c. The shaded areas in the figures highlight a region which should be easily distinguishable from the rest in order to allow for an early detection of excessive wear. Noticeably, the wear estimates are consistent to the wear profile data used in all scenarios, even for Scenarios 1 and 2 when the wear model is uncertain. For each $i$ th scenario, the wear estimates achieved at $k=100$ show good correspondence to $r_{\max }\left(\widetilde{F}^{i}, \widetilde{F}^{0}\right)$. These observations indicate positively to the viability of the determination of the wear related parameters based on historical data.

The wear estimates become smoother for larger $N$, which is in line with the simulation study of Section 3.1. For all scenarios, the larger wear estimates for $k>90$ allows for a distinction of the critical (shaded) regions. For Scenarios 0 and 1, the detection of a critical wear change could be achieved with a threshold set at the critical value of 35 . The same threshold would however give an early detection for Scenario 2. An early detection is understood as less critical than a failure but may lead to unnecessary maintenance actions. A more careful analysis of the wear estimates may therefore be needed in order to support accurate maintenance decisions.

The fact that the wear estimates do not differ much with $N$ might lead to the conclusion that $N=1$ should be used, but the behavior of the estimates degrade if non-optimal speed levels are chosen. To illustrate this, two wear estimates were achieved for Scenario 2 using only one measurement at $\dot{\varphi}=82.2$ and $\dot{\varphi}=133.5$. 


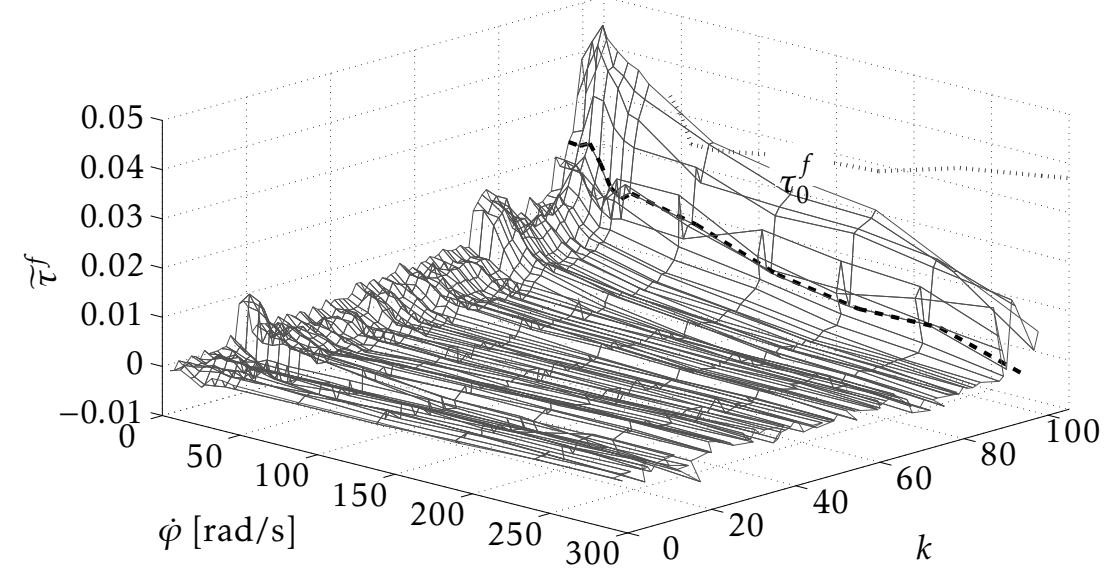

(a) Wear profile data $\widetilde{F}^{1}$.

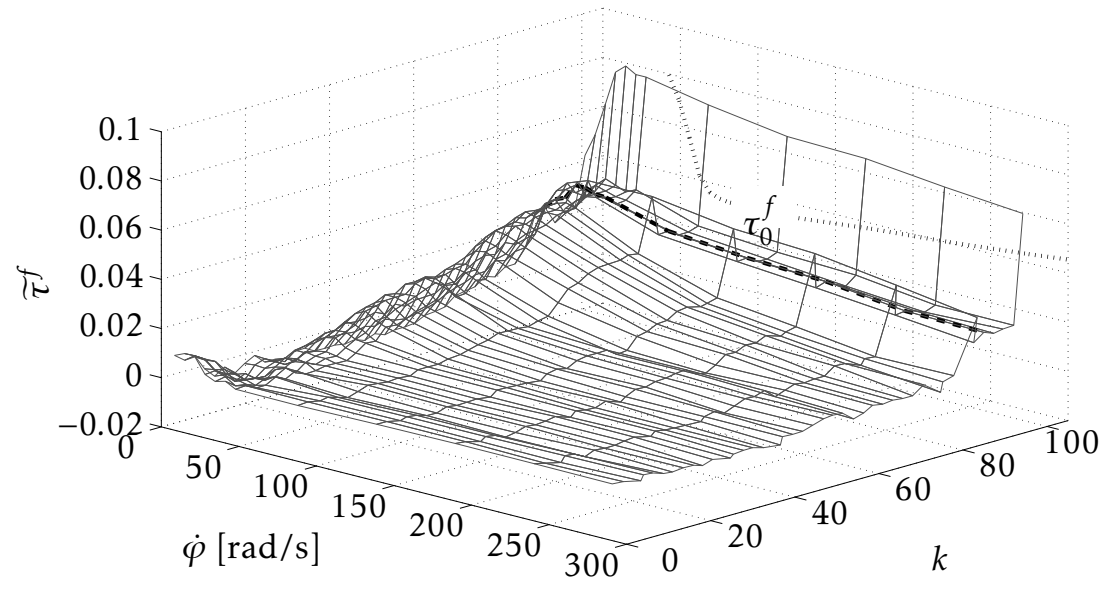

(b) Wear profile data $\widetilde{F}^{2}$.

Figure 9: Friction wear profile data used in Scenarios 1 (a) and 2 (b). The dashed lines indicate a critical wear level to be found. The dotted lines relate to the nominal friction curve $\tau_{0}^{f}$ that was removed from the friction data according to (6). 


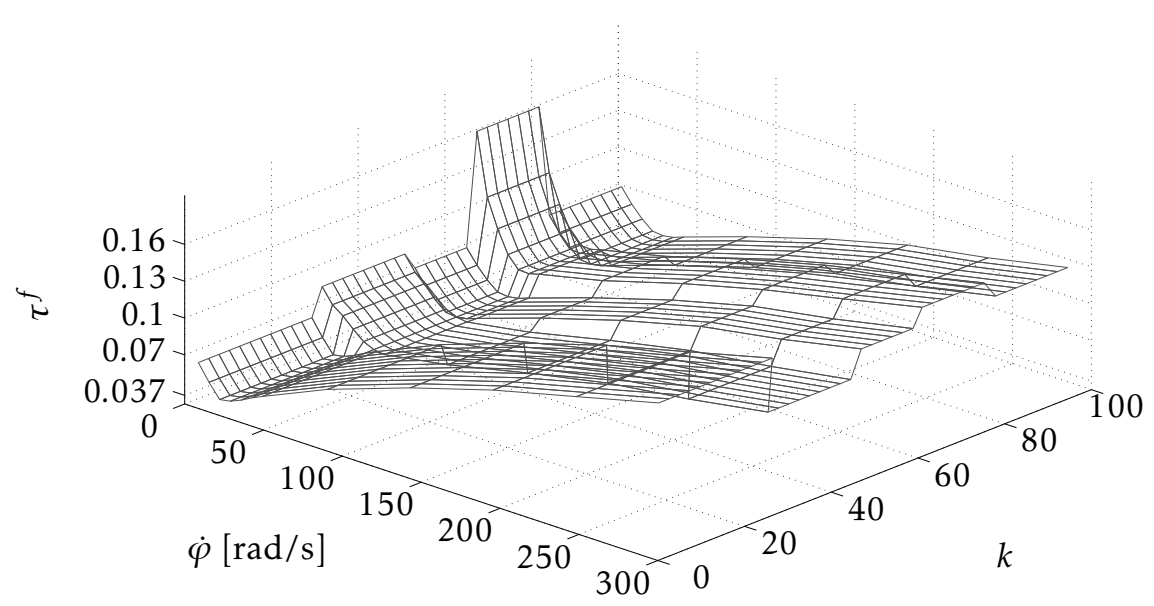

(a) Nominal friction behavior for all scenarios.

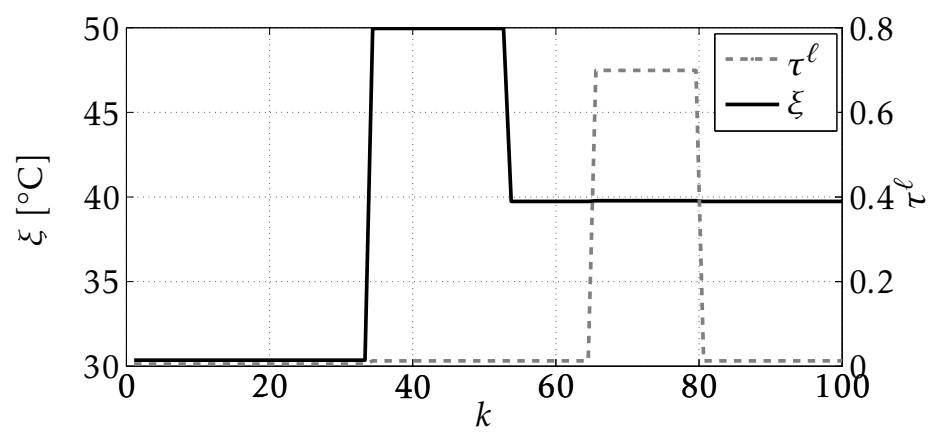

(b) Associated temperature, $\xi$, and load, $\tau^{\ell}$, values.

Figure 10: (a) Behavior of nominal friction as a function of $\dot{\varphi}$ and $k$ for the scenarios considered; an offset value corresponding to the smallest friction value in the data set was removed for a comparison to the wear profile data. The associated temperature and load values are shown in (b). 


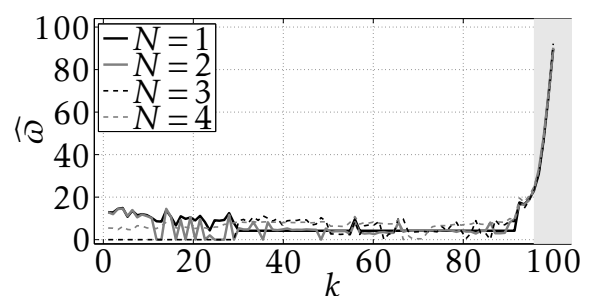

(a) Scenario 0 .

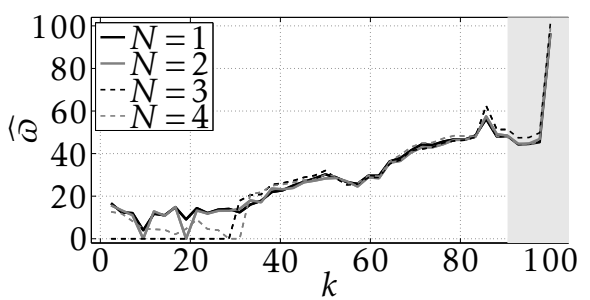

(c) Scenario 2.

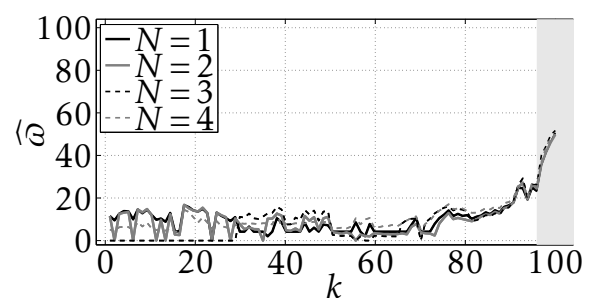

(b) Scenario 1.

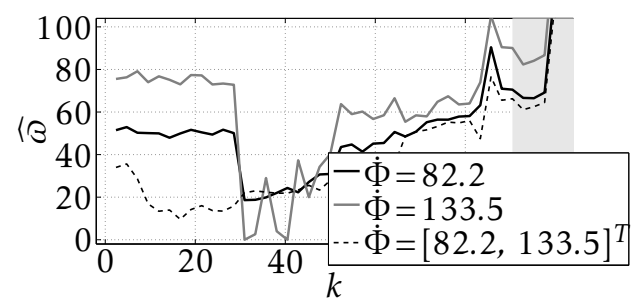

(d) Scenario 2 with non-optimal speed values.

Figure 11: Wear estimates for the different scenarios investigated. Figures (a) to (c) present the estimates for $N \in\{1,2,3,4\}$ using the optimal speed values. Figure (d) illustrates Scenario 2 when non-optimal speed values are used for $N=1$ and $N=2$. The shaded areas in the figures relate to a region where a detection should be made. 
As can be noticed, the wear estimates are considerably affected by changes in temperature when these speed values are used. However, when these two measurements are used together, the estimate improves significantly. The inclusion of measurements around the optimal speed values should also increase robustness to uncertainties in the wear model.

\section{Conclusions and Future Work}

A model-based maximum likelihood wear estimator was proposed based on a known friction model and constant-speed friction data collected from experiments. Because friction is considerably affected by other factors than wear, in particular temperature, a friction model that can describe these effects was suggested. Experiment design was considered to support the choice of speed levels for the friction data which reveals more information about wear. Simulations and case studies based on real data were considered to evaluate the approach. The wear estimates achieved in the studies showed a clear response to wear-related effects to friction, indicating that the approach may open up for condition based maintenance of industrial robots.

The studies presented here are restricted to one type of robot/gearbox and in an experimental verification performed in a lab. To verify the applicability of the proposed solutions in an industrial scenario, a more extensive experimental campaign is needed. Also interesting is to consider other types of variations and how they can affect the models and framework presented. For example, a change of lubricant may require the re-estimation of all or some of the friction parameters.

It should be stressed that different characteristics of the problem are expected for different devices, gearboxes and fault mechanisms. The results and discussion presented here may however provide useful guidance for those interested in using similar approaches for different devices. Of key importance to the proposed approach are the friction models used. The same ideas suggested for experiment design and wear identification can in principle be extended to other devices and model structures.

An extension to this work is to consider on-line wear estimation. This could perhaps be achieved by considering data from a friction observer, e.g. as presented in Ray et al. (2001); Marton (2011). The sensitivity of such approach to unmodeled phenomena, e.g. due to dynamic friction and external disturbances, should be considered carefully based on experiments performed on a real robot and different scenarios. 


\section{Bibliography}

F. Al-Bender and J. Swevers. Characterization of friction force dynamics. IEEE Control Systems Magazine, 28(6):64-81, 2008.

C. M. Bishop. Pattern Recognition and Machine Learning. Springer, New York, USA, 1st edition, 2006.

A. C. Bittencourt and S. Gunnarsson. Static friction in a robot joint- Modeling and identification of load and temperature effects. Journal of Dynamic Systems, Measurement, and Control, 134(5), July 2012.

A. C. Bittencourt, P. Axelsson, Y. Jung, and T. Brogårdh. Modeling and identification of wear in a robot joint under temperature disturbances. In Proceedings of the 18th IFAC World Congress, volume 18, Milan, Italy, August 2011.

D. Brambilla, L. Capisani, A. Ferrara, and P. Pisu. Fault detection for robot manipulators via second-order sliding modes. IEEE Transactions on Industrial Electronics, 55(11):3954-3963, November 2008.

F. Caccavale, P. Cilibrizzi, F. Pierri, and L. Villani. Actuators fault diagnosis for robot manipulators with uncertain model. Control Engineering Practice, 17(1): 146-157, 2009.

W. Chen. Fault detection and isolation in nonlinear systems: observer and energy-balance based approaches. Dissertation, Faculty of Eng. Automatic Control and Complex Systems, Duisubug-Essen University, October 2011.

S. E. Chick and M. B. Mendel. An Engineering basis for statistical lifetime models with an application to tribology. IEEE Transactions on Reliability, 45(2):208215, 1996.

A. De Luca and R. Mattone. Actuator failure detection and isolation using generalized momenta. In Proceedings of the 2003 IEEE International Conference on Robotics and Automation (ICRA), volume 1, pages 634-639, Taipei, Taiwan, September 2003.

K. De Moerlooze, F. Al-Bender, and H. Van Brussel. A generalised asperity-based friction model. Tribology Letters, 40:113-130, 2010.

W. E. Dixon, I. D. Walker, D. M. Dawson, and J. P. Hartranft. Fault detection for robot manipulators with parametric uncertainty: A prediction-error-based approach. IEEE Transactions on Robotics and Automation, 16(6):3628-3634, 2000.

I. Eski, S. Erkaya, S. Savas, and S. Yildirim. Fault detection on robot manipulators using artificial neural networks. Robotics and Computer-Integrated Manufacturing, 27(1):115-123, July 2011.

V. Filaretov, M. Vukobratovic, and A. Zhirabok. Observer-based fault diagnosis in manipulation robots. Mechatronics, 9(8):929-939, 1999. 
B. Freyermuth. An approach to model based fault diagnosis of industrial robots. In Proceedings of the 1991 IEEE International Conference on Robotics and Automation, volume 2, pages 1350-1356, April 1991.

S. C. Guo, M. H. Yang, Z. R. Xing, Y. Li, and J. Q. Qiu. Actuator fault detection and isolation for robot manipulators with the adaptive observer. Advanced Materials Research, 482 - 484(8):529-532, 2012.

P. Hamon, M. Gautier, and P. Garrec. Dynamic identification of robots with a dry friction model depending on load and velocity. In Proceedings of the 2010 IEEE/RSJ International Conference on Intelligent Robots and Systems, pages 6187-6193, Taipei, Taiwan, October 2010.

N. Kammerer and P. Garrec. Dry friction modeling in dynamic identification for robot manipulators: Theory and experiments. In Proceedings of the 2013 IEEE International Conference on Mechatronics, pages 422-429, 2013.

K. Kato. Wear in relation to friction - a review. Wear, 241(2):151-157, 2000.

A. R. Lansdown, A. L. Price, and J. Larsen-Basse. Materials to resist wear - a guide to their selection and use. Journal of Tribology, 109(2):379-380, 1987.

L. Marton. On-line lubricant health monitoring in robot actuators. In Proceedings of the 2011 Australian Control Conference (AUCC), pages 167-172, Melbourne, Australia, November 2011.

L. Marton. Energetic approach to deal with faults in robot actuators. In Proceedings of the 20th Mediterranean Conference on Control Automation (MED), pages 85-90, Barcelona, Spain, July 2012.

L. Marton and F. van der Linden. Temperature dependent friction estimation: Application to lubricant health monitoring. Mechatronics, 22(8):1078-1084, 2012.

M. McIntyre, W. Dixon, D. Dawson, and I. Walker. Fault identification for robot manipulators. IEEE Transactions on Robotics, 21(5):1028-1034, October 2005.

E. Olsson, P. Funk, and M. Bengtsson. Fault diagnosis of industrial robots using acoustic signals and case-based reasoning. In P. Funk and P. A. González Calero, editors, Advances in Case-Based Reasoning, volume 3155 of Lecture Notes in Computer Science, pages 13-15. Springer Berlin / Heidelberg, 2004.

B. Porat and B. Friedlander. Computation of the exact information matrix of Gaussian time series with stationary random components. IEEE Transactions on Acoustics, Speech and Signal Processing, 34(1):118-130, February 1986.

L. R. Ray, J. R. Townsend, and A. Ramasubramanian. Optimal filtering and Bayesian detection for friction-based diagnostics in machines. ISA Transactions, 40(3):207-221, 2001.

A. T. Vemuri and M. M. Polycarpou. A methodology for fault diagnosis in robotic systems using neural networks. Robotica, 22(04):419-438, 2004. 
J. A. Williams. Wear and wear particles - some fundamentals. Tribology International, 38(10):863-870, 2005. 


\section{Paper C}

\section{A Data-Driven Approach to Diagnostics of Repetitive Processes in the Distribution Domain}

Authors:

André Carvalho Bittencourt, Kari Saarinen, Shiva Sander-Tavallaey, Svante

Gunnarsson and Mikael Norrlöf .

Edited version of the paper:

A. C. Bittencourt, K. Saarinen, S. Sander-Tavallaey, S. Gunnarsson, and M. Norrlöf. A data-driven approach to diagnostics of repetitive processes in the distribution domain - Applications to gearbox diagnostics in industrial robots and rotating machines. Mechatronics, -(0):-, 2014. Available online.

Parts of this paper were previously published in:

A. C. Bittencourt, K. Saarinen, and S. Sander-Tavallaey. A data-driven method for monitoring systems that operate repetitively - applications to wear monitoring in an industrial robot joint. In Proceedings of the 8th IFAC SAFEPROCESS, volume 8, Mexico City, Mexico, 2012. 



\title{
A Data-Driven Approach to Diagnostics of Repetitive Processes in the Distribution Domain
}

\author{
André Carvalho Bittencourt* Kari Saarinen ${ }^{* *}$, Shiva Sander-Tavallaey ${ }^{* *}$, Svante \\ Gunnarsson* and Mikael Norrlöf***
}

${ }^{*}$ Dept. of Electrical Engineering,

Linköping University,

SE-581 83 Linköping, Sweden

\author{
${ }^{* *} \mathrm{ABB} \mathrm{AB}$ \\ Västerås, Sweden
}

\begin{abstract}
This paper presents a data-driven approach to diagnostics of systems that operate in a repetitive manner. Considering that data batches collected from a repetitive operation will be similar unless in the presence of an abnormality, a condition change is inferred by comparing the monitored data against an available nominal batch. The method proposed considers the comparison of data in the distribution domain, which reveals information of the data amplitude. This is achieved with the use of kernel density estimates and the Kullback-Leibler distance. To decrease sensitivity to disturbances while increasing sensitivity to faults, the use of a weighting vector is suggested which is chosen based on a labeled dataset. The framework is simple to implement and can be used without process interruption, in a batch manner. The approach is demonstrated with successful experimental and simulation applications to wear diagnostics in an industrial robot joint and for the diagnostics of gear faults in a rotating machine.
\end{abstract}

\section{Introduction}

In the manufacturing industry, preventive scheduled maintenance is a common approach used to improve equipment's safety, reliability and availability. This setup delivers high availability, reducing operational costs (e.g., small downtimes) with the drawback of high maintenance costs since unnecessary maintenance actions might take place. Condition based maintenance (СBM), "maintenance when required", can deliver a good compromise between operational and maintenance costs, reducing the overall cost of service. The extra challenge of СBM is to define methods to determine the condition of the equipment. This can be done by comparing the observed against the expected (known) behaviors of the system through an algorithm. The output of such algorithm is a test quantity, i.e., a 
quantity that can be tested to determine the current fault ${ }^{\mathrm{i}}$ state of the system (e.g., healthy/broken).

A common approach to generate test quantities is based on the use of residuals, i.e., test quantities that are achieved based on deviations between measurements and the output of a system model, see, e.g., Isermann (2011); Li and Zhou (2009). A system model is a map from input to output data and provides important information about the behavior of the system, facilitating the generation of test quantities. Different approaches for residual generation are based on, e.g., observers, parity-space and parameter identification. When a model of the system is not available or it is too costly to be developed, alternatives are still possible. These alternatives will typically require extra (redundant) sensory information or expert knowledge about the measured data, e.g., their nominal frequency content or the use of labeled data. Essentially, however, any method will attempt to generate quantities that can be used to infer the actual condition of the system given the available knowledge and observations, i.e., data.

The use of model-based approaches is common for the diagnostics of machines. In robotics, many approaches have been suggested based on the use of nonlinear observers, where the observer stability is typically guaranteed by analysis of the decay rate of a candidate Lyapunov function, see e.g. Filaretov et al. (1999); McIntyre et al. (2005); Caccavale et al. (2009); Guo et al. (2012); Brambilla et al. (2008); De Luca and Mattone (2003); Dixon et al. (2000). Observers can also be designed based only on data, without a first principles description of the system. Data-driven design of observers is typically based on subspace identification of linear models and have been suggested for fault detection by Ding et al. (2011); Wang et al. (2011); Dong et al. (2012); Yin et al. (2013). Parameter estimation is also a natural approach to model-based diagnostics because of the physical interpretation of the system parameters, see e.g. Bittencourt and Axelsson (2014); Freyermuth (1991); Marton and van der Linden (2012).

In cases where the data are ordered in time, signal-driven methods are common for machinery diagnostics. These are typically based on the use of integral transforms, e.g., Fourier, Radon, Karhunen-Loève or Wavelet. Each transform will enhance different properties in the transformed domain and are suitable depending on the characteristics of the signal, e.g., periodic, stationary, etc. The analysis of data in the frequency or time-frequency domains has found particular success for the monitoring of rotating machines, see, e.g., Taylor (1994); de Silva (2007); Fan and Zuo (2006); Halim et al. (2008); Sander-Tavallaey and Saarinen (2009); Isermann $(2006,2011)$. Some approaches have also been proposed for the diagnostics of industrial robots with the use of additional sensory information Olsson et al. (2004); Eski et al. (2011).

A common challenge to data-driven methods is that the characteristics of the data will vary depending on the operating points, which may complicate the determination of a fault presence. This is particularly restricting for an indus-

\footnotetext{
${ }^{\mathrm{i}} \mathrm{A}$ fault is defined as a deviation of at least one characteristic property of the system from the acceptable/usual/nominal condition.
} 
trial robot where the kinematic configuration of the robot may give varying load torques at the joints during motion. This shortcoming can be circumvented by considering data from a specific operation of the system, e.g., under repetition. A repetitive operation is found in various applications, e.g., in automated manufacturing. Repetition can also be forced with the execution of specific diagnostic routines but with the drawback of reduced availability. Much attention has been given recently to repetitive processes (Rogers et al., 2013a,b). Study of repetitive processes have mainly focused on control (Rogers et al., 2007; Sulikowski et al., 2004) and estimation problems (Aguilar-Lopez and Martinez-Guerra, 2007). Some approaches have been also suggested for model-based diagnostics, e.g., proposed by Wu et al. (2011).

In this paper, a data-driven approach is proposed for the diagnostics of systems that operate in a repetitive manner. It is considered that in case the condition of the system is nominal, data batches collected from a repetitive behavior of the system will be similar to each other and will differ if the condition changes. The comparison of a given data batch against a nominal one can thus be used to infer whether an abnormality is present. The test quantity proposed here relates to changes in the distribution of these batches of data. This is made possible with the use of kernel density estimators and the Kullback-Leibler distance between distributions. A distribution domain approach does not consider the dynamics of the system generating the data as is the case in, e.g., observer-based approaches. Nevertheless, as it will be presented, this leads to very simple diagnostic solutions that can perform well in practical setups.

The proposed framework was initially developed with the interest focused on the diagnostics of wear in industrial robots and a preliminary version of the work can be found in Bittencourt et al. (2012). Here, more aspects are covered, including approaches to detection, isolation and reduction of sensitivity to disturbances. More experimental and simulation results are also presented for the robotics application. An additional application is included for the diagnostics of rotating machinery based on vibration data collected from an accelerometer. The paper is organized as follows; a general presentation of data-driven diagnostics and repetitive systems is given in Section 2, followed by the presentation of the proposed approach for diagnostics in the distribution domain in Section 3. The applications are presented in Sections 4 and 5. Conclusions and future work are given in Section 6.

\section{Data-Driven Diagnostics and Repetitive Systems}

Consider a general system from which it is possible to extract a sequence of data batches,

$$
Y_{K}=\left[y_{1}, \cdots, y_{k}, \cdots, y_{K}\right] \text {, }
$$

where $y_{k}=\left[y_{k, 1}, \cdots, y_{k, n}, \cdots, y_{k, N}\right]^{T}$ denotes the $k$ th data batch in $\mathbb{R}^{N}$ (e.g. measurements, known inputs) with batch index $k$ and element index $n$. The sequence 
$y_{k}$ could have been generated as the result of deterministic and stochastic inputs, $z_{k}$ and $\boldsymbol{v}_{k}$ respectively, where $\boldsymbol{v}_{k}$ is unknown and $z_{k}$ can have known and unknown components. For example, the data generation mechanism could be modeled as

$$
y_{k}=\boldsymbol{h}\left(z_{k}, \boldsymbol{v}_{k}\right) \text {, }
$$

where $\boldsymbol{h}(\cdot)$ is an unknown function. The random inputs $\boldsymbol{v}_{k}$ are unknown, e.g., noise. The known components of $z_{k}$ include control inputs $\boldsymbol{u}_{k}$ and references $\boldsymbol{r}_{k}$. Unknown components of $z_{k}$ include disturbances $\boldsymbol{d}_{k}$ and faults $f_{k}$.

The objective is to define a data-driven framework for the generation of test quantities to determine the presence of a fault $f_{k}$. Because a data-driven approach depends on availability of data, it is assumed that data generated under no fault is available. Let $\mathcal{Y}^{0}=\left\{y_{k}: f_{k}=\mathbf{0}\right\}$ denote the set of data batches that were generated under no fault, the following assumption is made.

A-1 (Nominal data are available) A labeled sequence $y^{0} \in \mathcal{Y}^{0}$ is available.

The rationale is then to generate test quantities from the comparison of the nominal data $y^{0}$ (available from Assumption A-1) against the remaining sequences $y_{k}$. In order to generate test quantities for $y_{k}$ using the nominal data $y^{0}$, two basic questions arise:

Q-1 How to characterize a sequence $y_{k}$ ?

Q-2 How to compare the sequence $y^{0}$ against $y_{k}$ ?

The first question targets the issue of finding a data processing mechanism of $y_{k}$, written in a general form as $g(k) \triangleq g\left(y_{k}\right): \mathbb{R}^{N} \mapsto \mathbb{G}$ with domain $\mathbb{G}$, whose output are data features that can enhance the ability to discriminate the presence of nonzero $f_{k}$. Given the nominal data in the transformed domain, $g^{0} \triangleq g\left(y^{0}\right)$, test quantities, $q(k)$, can be achieved from the comparison between $g^{0}$ and $g(k)$. This is typically, but not necessarily, done with the use of a distance function and is represented as

$$
q(k) \triangleq d\left(g^{0}, g(k)\right): \mathbb{G} \times \mathbb{G} \mapsto \mathbb{R}_{0}^{+} .
$$

Different distances are possible depending on the domain $\mathbb{G}$. For example, for diagnostics of rotating machines $g(k)$ could be the spectra of $y_{k}$ and $d(\cdot, \cdot)$ a spectral distance, see, e.g., Basseville (1989) for more on distances between spectra.

\subsection{Detection, performance and isolation}

Let $\mathcal{Q}^{m, n}=\left\{q=d(g(i), g(j)): y_{i} \in \mathcal{Y}^{m}, y_{j} \in \mathcal{Y}^{n}\right\}$, then the set $\mathcal{Q}^{0,0}$ describes the behavior of the test quantity when no fault is present and $\mathcal{Q}^{0, f}$, where $\mathcal{Y}^{f}=\left\{y_{k}\right.$ : $\left.f_{k} \neq 0\right\}$, describes all possible faulty behaviors. A criterion for detectability of an abnormality is that $\mathcal{Q}^{0, f}$ is not completely contained in $\mathcal{Q}^{0,0}$, i.e., $\mathcal{Q}^{0, f} \backslash \mathcal{Q}^{0,0} \neq \emptyset$.

Since the test quantity $q(k)=d\left(g^{0}, g(k)\right)$ measures how far $g(k)$ is from the nomi- 
nal $g^{0}$, it is expected that it will remain close to zero if $q(k) \in \mathcal{Q}^{0,0}$ and to deviate to positive values if $q(k) \in \mathcal{Q}^{0, f}$. Suppose that it is possible to find a threshold $\hbar$ such that $q(k) \leq \hbar$ most of the times when $q(k) \in \mathcal{Q}^{0,0}$, a simple criterion for detection is then to consider a threshold check. Let $\mathcal{H}^{0}$ denote the hypothesis that no fault is present in $y_{k}$, i.e., $q(k) \in \mathcal{Q}^{0,0}$, and $\mathcal{H}^{1}$ denote the alternative hypothesis that a fault is present, i.e., $q(k) \in \mathcal{Q}^{0, f}$, then the decision mechanism is

$$
q(k) \underset{\mathcal{H}^{0}}{\stackrel{\mathcal{H}^{1}}{\gtrless} \hbar}
$$

and reads, decide for $\mathcal{H}^{0}$ if $q(k) \leq \hbar$ otherwise decide for $\mathcal{H}^{1}$.

The probabilities of error for this decision rule can be quantified given the probability distribution of the test quantity under the different hypotheses, denoted $p\left(q \mid \mathcal{H}^{0}\right)$ and $p\left(q \mid \mathcal{H}^{1}\right)$. The probability of a false detection $P_{f}$, i.e., deciding for a fault when none is present, and of correct detection $P_{d}$, i.e., deciding for a fault when it is present can be evaluated by

$$
P_{f}=\int_{\hbar}^{\infty} p\left(q \mid \mathcal{H}^{0}\right) \mathrm{d} q, \quad P_{d}=\int_{\hbar}^{\infty} p\left(q \mid \mathcal{H}^{1}\right) \mathrm{d} q .
$$

Notice that for a fixed $P_{f}$ there is an associated $\hbar$ (this is known as the NeymanPearson criterion for threshold selection (Van Trees, 2001)) and therefore a $P_{d}$. For a satisfactory performance of the test quantity, low $P_{f}$ and high $P_{d}$ are typically desirable.

Since this is a data-driven framework, data from the different fault types are needed a priori to address the isolation problem, i.e., determination of the fault type present. Let $\mathcal{Y}^{m}=\left\{y_{k}: f_{k}=f^{m}\right\}$ denote the set of data generated under the $m$ th fault type with $M$ possible fault modes, i.e., $m \in \mathbb{M}=\{1,2, \cdots, M\}$. For fault isolation it is assumed:

A-2 (Data from each fault type are available) A sequence $y^{m} \in \mathcal{Y}^{m}$ is available for each $m$ th fault type.

Once a fault is detected, the fault type can be determined by choosing $y^{m}$ that is closest to $y_{k}$ in the sense of the transformation $g(\cdot)$ and distance $d(\cdot, \cdot)$. That is, decide for presence of the $m^{*}$ fault type according to

$$
m^{*}=\arg \min _{m \in \mathbb{M}} d\left(g^{m}, g(k)\right) \text {. }
$$

\subsection{Repetitive systems}

The amount of overlap between the sets $\mathcal{Q}^{0,0}$ and $\mathcal{Q}^{0, f}$ relates to how difficult it will be to determine the presence of a fault. Ultimately, no overlap is present, i.e., $\left|\mathcal{Q}^{0,0} \cap \mathcal{Q}^{0, f}\right|=0$, when it is possible to determine the presence of a fault with no errors. This is particularly difficult to achieve because $y_{k}$, and thus $\mathcal{Q}^{0,0}$ and $\mathcal{Q}^{0, f}$, are affected by other inputs than faults. Since $\left|\mathcal{Q}^{0,0} \cap \mathcal{Q}^{0, f}\right| \leq \min \left|\mathcal{Q}^{0,0}\right|,\left|\mathcal{Q}^{0, f}\right|$, an 
attempt to try to reduce the overlaps is to reduce the size of the sets themselves. This can be achieved by restricting the behavior of the data $y_{k}$ or, conversely, by restricting the possible input space. This paper focuses on monitoring data collected from a repetitive operation of the system. Such data is achieved by a system that is commanded to execute the same trajectory $\mho$ for each batch of monitored data. For a system operating in closed loop, this type of data is represented by the set

$$
\mathcal{Y}_{r}=\left\{y_{k}: r_{k}=\mho\right\} \text {. }
$$

Alternatively, for open-loop systems the definition $\mathcal{Y}_{r}=\left\{y_{k}: \boldsymbol{u}_{k}=\mho \delta\right\}$ applies. Monitoring data $y_{k} \in \mathcal{Y}_{r}$ simplifies the problem since the behavior of the data will be more predictable. Examples of systems that behave repetitively are common in automation applications. A repetitive operation can also be forced in case the input commands can be chosen freely; for instance data can be collected based on the execution of scheduled diagnostic tests. This setup is also commonly found in vibration analysis and in signal-based diagnostics, where data are collected under particular operating conditions, e.g., of speed, load and acceleration.

Considering that faults are detectable for a system operating under repetition, there may still be overlaps between the sets $\mathcal{Q}_{r}^{0,0}$ and $\mathcal{Q}_{r}^{0, f}$ defined by $\mathcal{Y}_{r}^{0}=\mathcal{Y}^{0} \cap \mathcal{Y}_{r}$ and $\mathcal{Y}_{r}^{f}=\mathcal{Y}^{f} \cap \mathcal{Y}_{r}$. The presence of disturbances $\boldsymbol{d}_{k}$ and noise $\boldsymbol{v}_{k}$ may be important causes of overlaps. By collecting data in a controllable manner, it might be possible to ensure repetition of the disturbance term, i.e., to have $\boldsymbol{d}_{k}=\boldsymbol{d}$ for all batches. This is however too restrictive in many applications. Even if $\boldsymbol{d}_{k}=\boldsymbol{d}$ is possible, the noise components will always affect the data. To broaden the scope of the framework, a clear determination of the fault presence despite variations of $\boldsymbol{d}_{k}$ and $\boldsymbol{v}_{k}$ is desirable, leading to the question:

Q-3 How to handle non-repetitive disturbances $\boldsymbol{d}_{k}$ and noise $\boldsymbol{v}_{k}$ ?

Questions Q-1 to Q-3 are addressed in the next section which defines the suggested approach for diagnostics of repetitive systems in the distribution domain.

\section{A Distribution Domain Approach}

\subsection{Characterizing the data - Kernel Density Estimate}

The alternative pursued in this work is to consider the distribution of $y_{k}$, which contains information about the amplitude behavior of the data. Even though information contained in the ordering may be lost, this is a valid approach since the effects of faults often appear as changes in amplitude. Since data batches from a repetitive operation are considered, i.e., $y_{k} \in \mathcal{Y}_{r}$, it is expected that the data distribution will remain similar in case no fault is present. Because the mechanisms that generated the data are considered unknown, the use of a nonparametric estimate of the distribution of $y_{k}$ is a suitable alternative. A nonparametric estimate of the distribution $p(y)$ based on the data vector $y$ can be achieved from the empirical characteristic function. For a scalar random variable with probability den- 
sity function $p(y)$, the characteristic function $\vartheta(v): \mathbb{R} \rightarrow \mathbb{C}$ is defined as (Durrett, 2010):

$$
\vartheta(v)=\mathbb{E}\left[e^{\jmath v y}\right]=\int_{-\infty}^{\infty} e^{\jmath v y} p(y) \mathrm{d} y=\mathbf{F}^{-1}\{p(y)\} 2 \pi,
$$

where $\mathbf{F}^{-1}\{\cdot\}$ is the inverse Fourier transform. So the density function can be found from the characteristic function through its Fourier transform. Following Parzen (1962), given the sample $\mathbf{y}=\left[y_{1}, \cdots, y_{N}\right]^{T}$, the empirical estimate of $\vartheta(v)$ is given by

$$
\widehat{\vartheta}(v)=\frac{1}{N} \sum_{n=1}^{N} e^{j v y_{n}},
$$

the objective is then to estimate the density function from $\widehat{\vartheta}(v)$. This is essentially a spectrum estimation problem. A direct estimation of the density function from the Fourier transform of $\widehat{\vartheta}(v)$ will however lead to an estimate with variance that does not decrease with $N$ (Ljung, 1999, Section 6.4). To avoid this problem, the empirical estimate of the characteristic function is multiplied with a weighting function $\psi_{h}(v)=\psi(h v)$. The weighting function is typically symmetric, satisfying $\psi(0)=1$ and tends to zero when $v$ tends to infinity. The density estimate is then given by

$$
\begin{aligned}
\widehat{p}(y) & =\frac{1}{2 \pi} \mathbf{F}\{\widehat{\vartheta}(v) \psi(h v)\}=\frac{1}{2 \pi} \int_{-\infty}^{\infty} e^{-\jmath v y} \widehat{\vartheta}(v) \psi(h v) \mathrm{d} v \\
& =\frac{1}{2 \pi} \int_{-\infty}^{\infty} \frac{1}{N} \sum_{n=1}^{N} e^{\jmath v\left(y_{n}-y\right)} \psi(h v) \mathrm{d} v=\frac{1}{N h} \sum_{n=1}^{N} \frac{1}{2 \pi} \int_{-\infty}^{\infty} e^{\jmath v\left(\frac{y_{n}-y}{h}\right)} \psi(h v) \mathrm{d}(h v) \\
& =\frac{1}{N h} \sum_{n=1}^{N} \kappa\left(\frac{y_{n}-y}{h}\right)=\frac{1}{N} \sum_{n=1}^{N} \kappa_{h}\left(y-y_{n}\right),
\end{aligned}
$$

where $\kappa_{h}(y) h=\mathbf{F}^{-1}\left\{\psi_{h}(v)\right\}$. The resulting estimate is known as a kernel density estimate (KDE) and can also be generalized to the multidimensional case (Cacoullos, 1966). For a given sequence $y_{k}$ the resulting KDE estimate is denoted $\widehat{p}_{k}(y)$ and from the notation of Section $2, g(k)=\widehat{p}_{k}(y)$, i.e., the KDE is the data feature.

The function $\kappa_{h}(y)$ is a kernel function, satisfying $\kappa_{h}(\cdot) \geq 0$ and that integrates to one. Typical kernel functions and their Fourier transforms are shown in Figure 1. The bandwidth parameter $h$ controls the smoothness of the resulting estimate, increasing the smoothness for larger values of $h$. When $h \rightarrow 0$, the kernel function approaches a Dirac delta and the resulting estimate will be a set of impulses located at the data points. In this work, a Gaussian kernel is used with $h$ optimized for Gaussian distributions as described by Bowman and Azzalini (1997). For a detailed treatment of kernel density estimators and criteria/methods for choosing $h$ see Parzen (1962); Cacoullos (1966); Bowman and Azzalini (1997); 


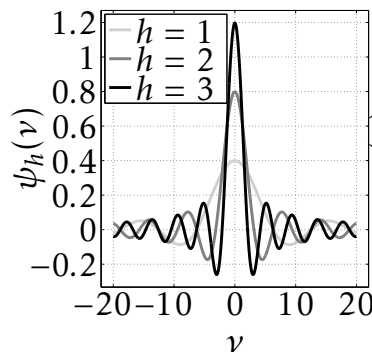

(a) Uniform.

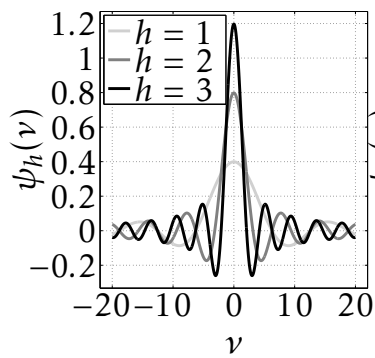

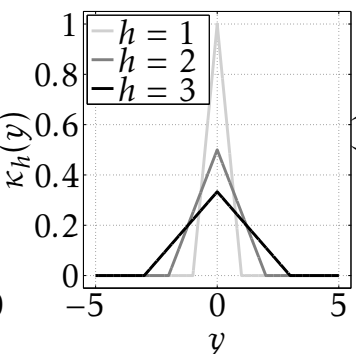

(b) Triangular.

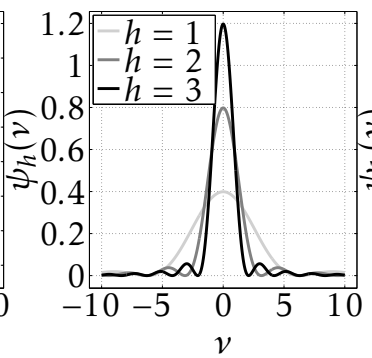

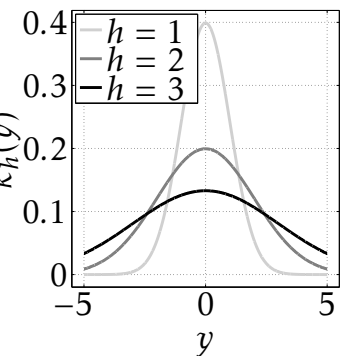

(c) Gaussian.

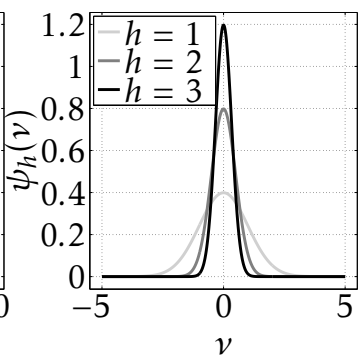

Figure 1: Kernel functions (upper row) and their respective Fourier transforms (bottom row).

Jones and Henderson (2009).

\subsection{Comparing sequences - Kullback-Leibler distance}

In statistics and information theory, the Kullback-Leibler divergence (KLD) is commonly used as a measure of difference between two probability distributions. For two continuous distributions on $y, p^{A}(y)$ and $p^{B}(y)$, it is defined as (Reid and Williamson, 2011)

$$
D_{\mathrm{KL}}\left(p^{A} \| p^{B}\right) \triangleq \int_{-\infty}^{\infty} p^{A}(y) \log \frac{p^{A}(y)}{p^{B}(y)} \mathrm{d} y
$$

The KLD satisfies $D_{\mathrm{KL}}\left(p^{A} \| p^{B}\right) \geq 0$ (Gibbs inequality), with equality if and only if $p^{A}(y)=p^{B}(y)$. The KLD is not a distance as it is not symmetric in general. The quantity

$$
\mathrm{KL}\left(p^{A}, p^{B}\right) \triangleq D_{\mathrm{KL}}\left(p^{A} \| p^{B}\right)+D_{\mathrm{KL}}\left(p^{B} \| p^{A}\right),
$$

is however symmetric and is known as the symmetric Kullback-Leibler divergence or Kullback-Leibler distance. An answer to Question Q-2 can therefore be given with the use of the KL distance defined in (11). From Assumption A-1, fault-free data are always available, so that $y^{0}$ is known and $\widehat{p}^{0}(y)$ can be computed. The KL distance can thus be used as test quantities, i.e., $q(k)=\operatorname{KL}\left(\widehat{p}^{0}, \widehat{p}_{k}\right)$, 
remaining close to 0 in case $\widehat{p}^{0}(y)$ is close to $\widehat{p}_{k}(y)$ and otherwise deviating to positive values.

\subsection{Handling non-repetitive disturbances and noise - data weighting}

One approach to address Question Q-3 is to weight the raw data $y_{k}$ according to prior knowledge of the effects of faults, disturbances and noise in order to give more relevance to parts of the data that relate to a fault. The approach considered here will assume availability of a labeled dataset, where the fault status (present or not) is known to each batch $y$ and is the same to each of its elements $y_{n}$. The disturbance and noise components should contain variations that are expected to be found during the system's operation.

The labeled dataset is given by

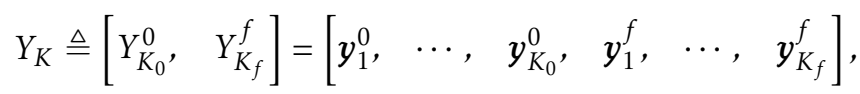

with $K_{0}$ fault-free data, $y^{0} \in \mathcal{Y}_{r}^{0}$, and $K_{f}=K-K_{0}$ faulty data $y^{f} \in \mathcal{Y}_{r}^{f}$. Each batch $y_{k}$ is weighted as

$$
\bar{y}_{k} \triangleq w \odot y_{k},
$$

where $\odot$ denotes the Hadamard product (element-wise multiplication). This yields the weighted dataset

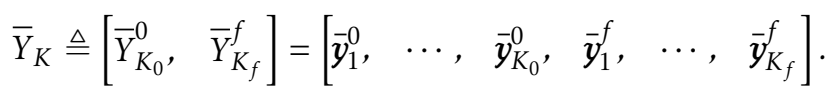

The objective is to choose $w$ to maximize the sensitivity to faults while decreasing sensitivity to disturbances and noise.

In this work, simple criteria are considered in a compromise to explicit solutions. As it will be shown, the results are related to linear discriminant analysis (LDA) used for dimensionality reduction and classification problems, see, e.g., Bishop (2006). In LDA, the inner product $w^{T} y$ is used instead of the Hadamard product and the objective is to reduce the dimension of the data. While the data dimension is reduced in LDA, the use of the Hadamard product keeps the data dimensionality and therefore the KDE can still be computed, yielding the estimates $\widehat{\bar{p}}_{k}$.

Notice that once the weights are chosen, the same vector $w$ is used for new data batches. For consistency, it is thus required that the data sequences are synchronized. This can however be overcome in case the weights are strongly correlated to measured data. In such case, an approximate function can be used to describe the weights relation to the data, e.g., described as a static function $w(\cdot)$. The use of such representation of the weights is illustrated in Section 4.2. 


\section{Choosing $w$ - Linear Discriminant Analysis}

A criterion is to choose $w$ that maximizes the distance between the averages of the weighted datasets $\bar{Y}_{K_{0}}^{0}$ and $\bar{Y}_{K_{f}}^{f}$. The average weighted vector for $Y_{K_{0}}^{0}$ is

$$
\bar{\mu}^{0}=\frac{1}{K_{0}} \sum_{k=1}^{K_{0}} w \odot y_{k}^{0}=w \odot \underbrace{\left(\frac{1}{K_{0}} \sum_{k=1}^{K_{0}} y_{k}^{0}\right)}_{\triangleq \boldsymbol{\mu}^{0}}=w \odot \mu^{0}
$$

and similarly $\bar{\mu}^{f}=w \odot \mu^{f}$. Based on the resulting average vectors, a natural criterion would be to find their kernel density estimates and choose $w$ that maximizes the KL distance between them. A general solution to this problem is not possible since it depends on how the KDE is computed (e.g. the kernel function chosen) and optimization over (11). Instead, a simpler criterion is considered, where $w$ is chosen to maximize the difference between the means of $\bar{\mu}^{f}$ and $\bar{\mu}^{0}$, i.e.,

$$
\frac{1}{N} \sum_{n=1}^{N} w_{n} \mu_{n}^{f}-\frac{1}{N} \sum_{n=1}^{N} w_{n} \mu_{n}^{0}=\frac{1}{N} w^{T}\left(\mu^{f}-\mu^{0}\right) .
$$

Constraining $w$ to unit length $w^{T} w=1$ (otherwise the criterion can be made arbitrarily large), it is possible to find that (16) is maximized for (see e.g. (Bishop, 2006, Exercise 4.4)),

$$
w^{*} \propto\left(\mu^{f}-\mu^{0}\right) .
$$

The criterion (16) does not account for the variability found within each dataset, e.g., caused by disturbances and noise. An alternative is to consider maximum separation between the means of $\bar{\mu}^{0}$ and $\bar{\mu}^{f}$ while giving small variability within each dataset and thus avoiding overlaps. The sample covariance for $\bar{Y}_{K_{0}}^{0}$ (and similarly for $\bar{Y}_{K_{f}}^{f}$ ) is given by

$$
\begin{aligned}
\bar{\Sigma}^{0} & =\frac{1}{K_{0}} \sum_{k=1}^{K_{0}}\left(w \odot y_{k}-w \odot \mu^{0}\right)\left(w \odot y_{k}-w \odot \mu^{0}\right)^{T} \\
& =\frac{1}{K_{0}} \sum_{k=1}^{K_{0}}\left(w \odot\left(y_{k}-\mu^{0}\right)\right)\left(w \odot\left(y_{k}-\mu^{0}\right)\right)^{T} \\
& =\left(w w^{T}\right) \odot \underbrace{\frac{1}{K_{0}} \sum_{k=1}^{K_{0}}\left(y_{k}-\mu^{0}\right)\left(y_{k}-\mu^{0}\right)^{T}}_{\triangleq \Sigma^{0}}=\left(w w^{T}\right) \odot \Sigma^{0} .
\end{aligned}
$$

The total covariance is thus $\bar{\Sigma} \triangleq\left(w w^{T}\right) \odot\left(\Sigma^{f}+\Sigma^{0}\right)$. Considering the trace of $\bar{\Sigma}$ as a measure for the variability of the weighted data, an alternative criterion to (16) 
is to consider maximization of the ratio

$$
\frac{\left(w^{T}\left(\mu^{f}-\mu^{0}\right)\right)^{2}}{\operatorname{tr}(\bar{\Sigma})}=\frac{w^{T}\left(\mu^{f}-\mu^{0}\right)\left(\mu^{f}-\mu^{0}\right)^{T} w}{\sum_{n=1}^{N} w_{n}^{2}\left(\left[\Sigma^{0}\right]_{n n}+\left[\Sigma^{f}\right]_{n n}\right)}=\frac{w^{T}\left(\mu^{f}-\mu^{0}\right)\left(\mu^{f}-\mu^{0}\right)^{T} w}{w^{T}\left(I \odot\left(\Sigma^{f}+\Sigma^{0}\right)\right) w} .
$$

This is a special case of the Fisher criterion in LDA. It can be shown, see e.g. (Bishop, 2006, Section 4.1.4), that the solution for this problem is given by

$$
w^{*}=\left(I \odot\left(\Sigma^{f}+\Sigma^{0}\right)\right)^{-1}\left(\mu^{f}-\mu^{0}\right) .
$$

That is, each weight $w_{n}^{*}$ is proportional to the ratio between the average changes caused by faults, $\mu_{n}^{f}-\mu_{n}^{0}$, and the total variability found in the data, $\left[\Sigma^{0}\right]_{n n}+$ $\left[\Sigma^{f}\right]_{n n}$, caused by disturbances and noise.

\section{Wear Monitoring in an Industrial Robot Joint}

In this first application, the objective is to determine the presence of excessive levels of wear, $\omega$, in the gearbox of a robot joint. Because increased wear levels may lead to increased friction in the gearbox, it is possible to monitor friction to infer about wear. Since the friction torques must be overcome by the applied motor torques, $\tau$, during its operation, it is possible to extract information about friction from available data. Friction is however not only affected by wear, but also by unknown disturbances, such as variations of load torques, $\tau^{\ell}$, and the lubricant temperature, $\xi$, see Figure $2^{i}$. While it may be simpler to ensure constant load conditions, temperature is the result of complicated losses mechanisms in the joint and heat exchanges with the environment which are difficult to control. The effects of $\tau^{\ell}$ and $\xi$ to friction are in fact comparable to those caused by $\omega$ (recall Figure 2) and the problem is therefore challenging. In Bittencourt and Axelsson (2014), a model-based approach was suggested for this problem based on the identification of a wear parameter from friction data collected under dedicated experiments. A shortcoming with this approach lies in the need of a detailed friction model, which requires a considerable amount of experiments to be found. The data-driven approach suggested here is considered as an alternative which requires little design effort since no model development is needed.

Since $\tau$ is affected by friction, and thus by wear, torque (current ${ }^{\mathrm{ii}}$ ) data are considered for the generation of test quantities. The monitored data are collected from repetitive executions of a trajectory $\mho$. Relating to the notation introduced in Section 2, the deterministic unknown input of interest, $f$, is the wear level $\omega$ and the monitored data, $y$, is $\tau$ which is affected by disturbances, $d$, caused by load $\tau^{\ell}$ and temperature $\xi$ and by measurement noise $\boldsymbol{v}$. A trajectory, $\mho$, is a known deter-

\footnotetext{
${ }^{\mathrm{i}}$ Throughout the paper, all torque quantities are normalized to the maximum allowed torque and are therefore dimensionless.

${ }^{i i}$ In the application, a torque estimate based on a constant relationship between current measurements is used. This simplification is commonly used for control purposes since the current controller has much faster dynamics compared to the dynamics of the robot arm and such estimate is therefore perceived as the control input signal.
} 


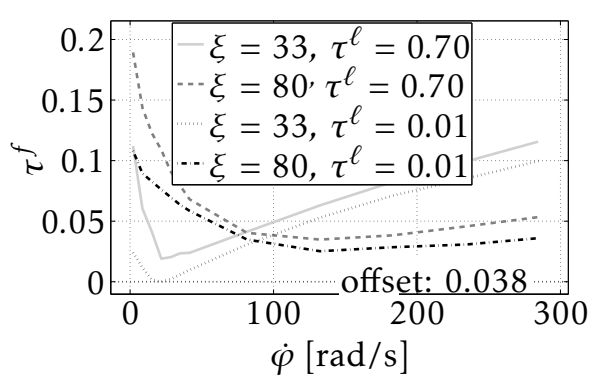

(a) Effects of load and temperature.

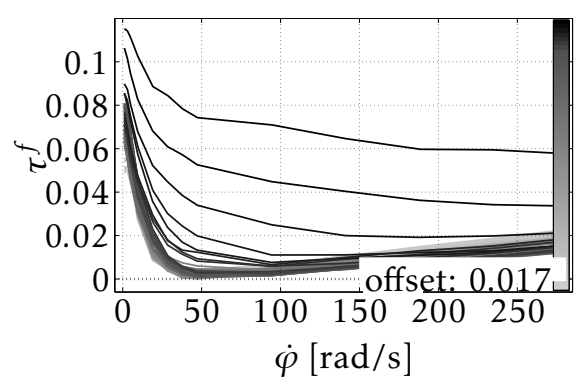

(b) Effects of wear.

Figure 2: Friction levels $\tau^{f}$ in a robot joint as a function of motor speed $\dot{\varphi}$ under different conditions of wear, temperature and load. The offset values were removed for a comparison, their values are shown by the dotted lines. The data were collected from similar gearboxes and are directly comparable. Notice the different scales used and the larger amplitude of effects caused by temperature and load compared to those caused by wear. In (b), the colormap relates to the length of accelerated wear tests during which the curves were registered.

ministic sequence used as a reference to the motion control, i.e., it relates to $r$. In many applications, the same trajectory is executed over and over again, ensuring a repetitive behavior of the robot. For the results presented here, data collected from the execution of a trajectory $\mho$ based on a test-cycle are used. Torque data collected form this trajectory can be seen in Figure 3a. Nominal torque data, $\tau^{0}$, are achieved from the execution of $\mho \checkmark$ when the gearbox is new and no significant wear is present.

Section 4.1 presents experimental results for the wear monitoring problem when the changes in disturbances are kept small. In Section 4.2, temperature disturbances are introduced in simulation studies and the use of the weights described in Section 3.3 is illustrated to reduce sensitivity to disturbances.

\subsection{Experimental studies under constant disturbances}

Accelerated wear tests were performed with ABB IRB 6620 industrial robots with the objective of studying the wear effects. In an accelerated wear test, the robot is run under high load and stress levels for several months or years until the wear levels become significant and maintenance is required. Throughout the tests, the trajectory $\mho$ was executed regularly a total of $K$ times yielding a dataset $\left[\tau_{0}, \cdots, \tau_{K-1}\right]$. The data were collected from axis two of the robot which is equipped with a rotary vector gearbox type. The experiments were performed in a lab, in a setup to avoid temperature variations ${ }^{i}$ and no load variations were present. It is thus considered that the disturbances had a repetitive behavior, i.e.,

\footnotetext{
${ }^{\mathrm{i}}$ The environment temperature was controlled and the experiments were only performed after the robot temperature was expected to be in equilibrium with the environment.
} 
$\boldsymbol{d}_{k}=\boldsymbol{d}$ over all batches. The data batch taken from the start of the operation of a gearbox is considered to be fault-free and is labeled as nominal, $\tau^{0}$. The test quantities $q(k)=\mathrm{KL}\left(\widehat{p}^{0}, \widehat{p}_{k}\right)$ are computed for $k=1, \ldots, K-1$ and used to indicate presence of faults. Data collected from two accelerated wear tests are considered here. For an illustration of the wear behavior during the experiments, the friction levels of the joint were estimated using a dedicated experiment (see Bittencourt and Gunnarsson (2012) for a description of such experiment) at each $k$ th execution of $\mho$ and are shown as function of motor speed $\dot{\varphi}$.

For the first case, displayed in Figure 3, $K=36$ batches of data are considered. From analysis of the friction levels in Figure $3 c$, it is possible to note that wear only starts to considerably affect friction after $k=25$. The effects of wear to the torque sequences, shown in Figure $3 a$, appear as small variations in amplitude due to increased friction. The variations in the torque sequences are more easily distinguishable in the distribution domain as seen in Figure 3b. Wear affects the location and size of the peaks of the distributions. Notice further that the distributions are similar for $k \leq 25$ when the robot condition has not significantly changed. The resulting test quantity, shown in Figure $3 d$, shows a clear response to the changes in friction, remaining close to 0 for $k \leq 25$ and increasing thereafter. To allow for CBM, it is considered that, in this test, a fault should be detected before $k=30$. Using data for $k \leq 25$, the mean and standard deviation for the (considered) nominal behavior of the test quantity are estimated as $\left[\mu_{0}, \sigma_{0}\right]=\left[1.1910^{-2}, 5.0910^{-3}\right]$. The dashed line in Figure $3 \mathrm{~d}$ shows the value of $\hbar=\mu_{0}+3 \sigma_{0}$ which could be used as a threshold.

The second case, shown in Figure 4, illustrates the situation where a gearbox is replaced after a wear related failure takes place. A total of $K=111$ data batches are collected during accelerated wear tests using the same test-cycle. A gearbox failure occurs at $k=73$ when it is replaced by a new one. The friction curves related to the faulty gearbox are shown in Figure $4 c$, where can be noticed that the changes due to wear start to appear around $k=64$. The related distribution estimates for this gearbox are shown in Figure $4 \mathrm{a}$ where a similar behavior as in the previous case can be noticed, with changes in the size and position of the distributions' peaks. The data densities for the replaced gearbox can be seen in Figure $4 \mathrm{~b}$ where it is possible to notice that no significant variations are present. The test quantity is shown in Figure $4 \mathrm{~d}$, where, as in the previous case, $\hbar=\mu_{0}+3 \sigma_{0}$. The filled circle highlights the moment when the gearbox was replaced. As can be seen in these studies, an early detection of the increased wear is made possible with the use of the proposed test quantity, allowing for СВM.

\subsection{Simulation studies under temperature disturbances}

Simulation studies were carried out to illustrate the ideas to reduce sensitivity to disturbances and noise presented in Section 3.3. The use of simulations allow for more detailed studies of the effects of the disturbances compared to what could be achieved based on experiments in a feasible manner. The simulation model is based on the two link manipulator with elastic gear transmission presented in the 


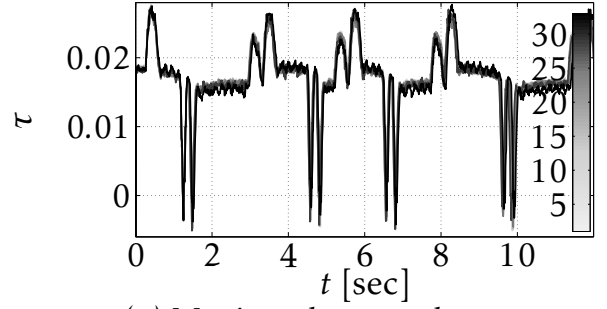

(a) Monitored torque data.

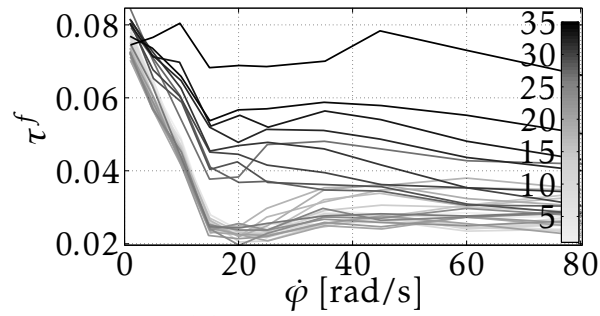

(c) Friction curves.

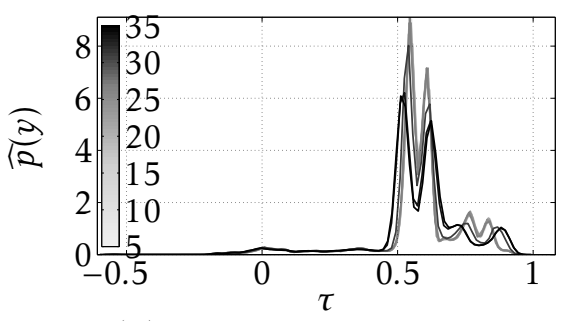

(b) Estimated distributions.

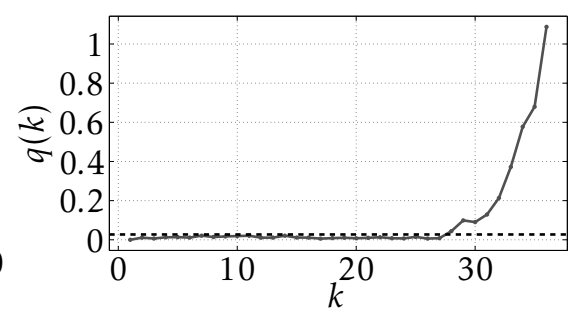

(d) Test quantity, $q(k)=K L\left(\widehat{p}^{0}, \widehat{p}_{k}\right)$.

Figure 3: Monitoring of a wear fault in an industrial robot joint under accelerated wear tests and controlled load and temperature disturbances. The colormaps relate to the experiment length $k$. The friction changes caused by wear were estimated during the experiments and are shown in (c) for a comparison. The monitored torque data from the execution of the trajectory $v$ are shown in (a), their respective KDEs were computed using a Gaussian kernel and are shown in (b). At $k=0$, it is considered that the robot is fault-free and the test quantity given by $q(k)=K L\left(\widehat{p}^{0}, \widehat{p}_{k}\right)$ is shown in $(d)$ where the dashed line represents an upper limit for its nominal behavior. Notice the clear response of the test quantity to the wear changes. 


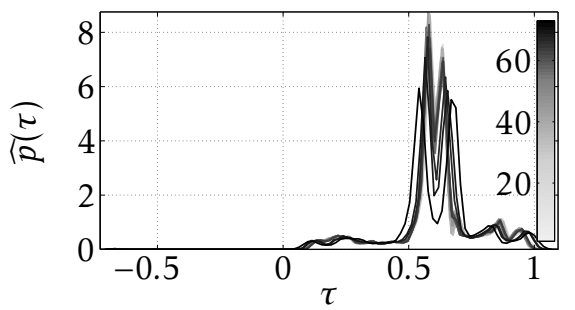

(a) Estimated distributions for $0 \leq k \leq$ 72 .

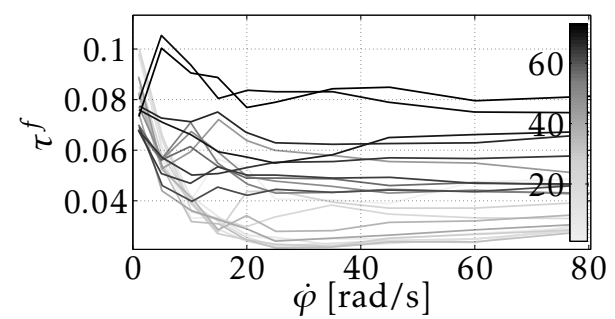

(c) Friction curves for $0 \leq k \leq 72$.

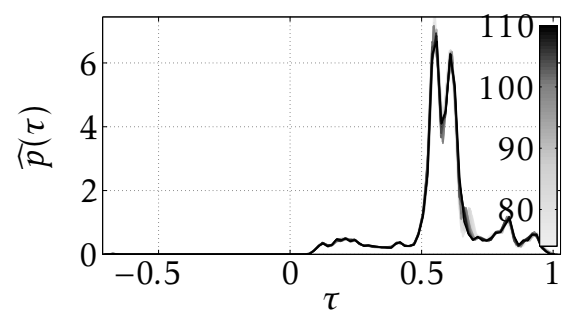

(b) Estimated distributions for $73 \leq k \leq$ 110.

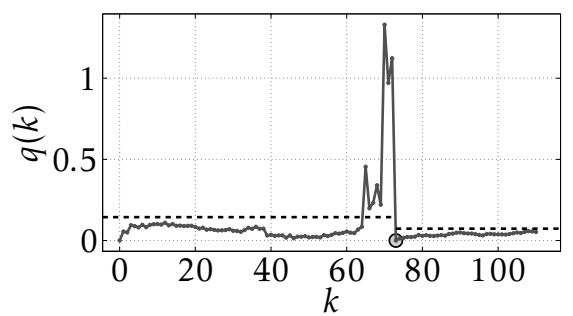

(d) Test quantity, $q(k)=K L\left(\widehat{p}^{0}, \widehat{p}_{k}\right)$.

Figure 4: Monitoring of a wear fault in an industrial robot joint under accelerated wear tests and controlled load and temperature disturbances. Data collected from the same trajectory $\mho$ used in Figure 3 are considered. A wear fault develops in the gearbox from $k=0$ to $k=72$, whereafter the faulty gearbox is replaced by a new one. The data distribution estimates for the faulty gearbox are shown in (a), which present similar behaviors as for the previous case, recall Figure $3 b$; the respective friction curves are shown in (c). The data distributions for the new gearbox are shown in (b), where only small deviations are visible. The nominal data are assigned at the start of operation for the gearboxes at $k=0$ and at $k=73$. The resulting test quantities are shown in Figure (d), with a clear response to the friction changes and a regular behavior when no fault is present; the circle in the figure highlights when the gearbox replacement took place and the dashed lines represent an upper limit for the nominal behavior of the test quantity. 
benchmark problem of Moberg et al. (2008). With the objective of studying friction changes related to wear in a robot joint, the static friction model described in Bittencourt and Axelsson (2014) is included in the simulation model. The friction model included was developed from empirical studies in a robot joint and describes the effects of angular speed $\dot{\varphi}$, manipulated load torque $\tau^{\ell}$, temperature $\xi$, and wear $\omega$.

\section{Finding the weights $w$}

According to the procedures described in Section 3.3, a labeled dataset is needed in order to find the optimal weights. The dataset is achieved here based on simulations of the same test-cycle trajectory $\mho$ used in Section 4.1. Each labeled dataset $Y_{K_{0}}^{0}$ and $Y_{K_{f}}^{f}$ contains $K_{0}=K_{f}=100$ batches with torque data generated from

$$
\begin{aligned}
& \mathcal{Y}_{r}^{0}=\left\{\tau_{k}: \omega_{k}=0, \xi_{k}=\xi^{(k)}, \mho_{k}=\mho\right\} \\
& \mathcal{Y}_{r}^{f}=\left\{\tau_{k}: \omega_{k}=\omega_{c}, \xi_{k}=\xi^{(k)}, \mho_{k}=\mho\right\}
\end{aligned}
$$

respectively, where $\omega_{c}=35$ is a wear level considered critical to generate an alarm (see Bittencourt and Axelsson (2014) for details of the wear model). Here, $\xi_{k}$ is considered constant for each $k$ th run with value $\xi^{(k)}$ determined randomly from a uniform distribution

$$
\xi^{(k)} \sim \mathcal{U}\left[\underline{\xi}, \underline{\xi}+\Delta_{\xi}\right]
$$

with lower level given by $\underline{\xi}=30^{\circ} \mathrm{C}$ and width $\Delta_{\xi}=40^{\circ} \mathrm{C}$. This assumption is carried out for analysis purposes and allows for great variations of temperature.

The optimal weights given in (17) and (18) depend on the average changes found in the data, $\mu_{n}^{f}-\mu_{n}^{0}$, and the total variability, $\left[\Sigma^{0}\right]_{n n}+\left[\Sigma^{f}\right]_{n n}$. These quantities are computed based on the labeled dataset and are displayed in Figure $5 \mathrm{a}$ as a function of the motor speed $\dot{\varphi}$. As can be seen, the optimal weights present a strong correlation with $\dot{\varphi}$. This is not a surprise since the effects of $\omega$ and $\xi$ depend on $\dot{\varphi}$, recall Figure 2. In the same figure, worst case estimates along speed are also shown (solid lines), giveb by $\mu_{n}^{f}-\mu_{n}^{0}$ closest to zero and largest $\left[\Sigma^{0}\right]_{n n}+$ $\left[\Sigma^{f}\right]_{n n}$. Figure $5 \mathrm{~b}$ presents the ratio for such worst case estimates, which are considered as the optimal weights according to (18). The solid line in Figure 5b is a function approximation of the optimal weights given by

$$
w(\dot{\varphi})=\operatorname{sech}(\beta \dot{\varphi}) \tanh (\alpha \dot{\varphi})
$$

with $\alpha=1.4510^{-2}$ and $\beta=4.5510^{-2}$. The parametrization of the weight vector as a function of $\dot{\varphi}$ allows for a more general use of the optimal weights since the same weighting function can be used for other trajectories. The optimal weighting function selects a speed region that is more relevant for wear monitoring, giving more emphasis to data in low to intermediate speed regions. A similar behavior was also found in Bittencourt and Axelsson (2014) for the achievable quality of a wear estimate for different speeds. 


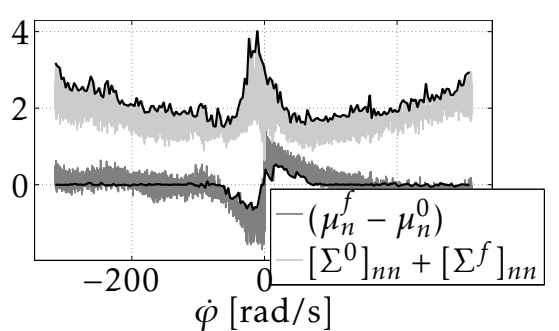

(a) Average effects.

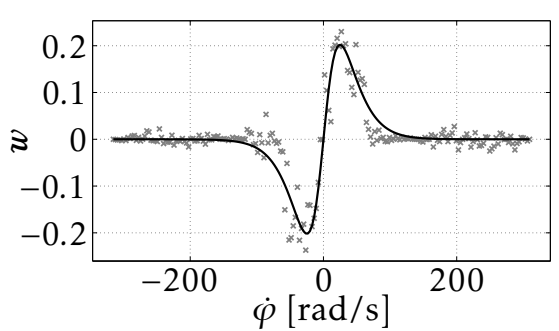

(b) Optimal weights.

Figure 5: Choice of optimal weights $w$. The effects of disturbances by temperature and faults are shown in (a), together with worst case estimates (black lines). The optimal weights for the worst case estimates are shown in (b) together with a function approximation (solid). Notice how the optimal region for wear monitoring is concentrated in a narrow speed range.

\section{Improvements in detection performance}

To illustrate the possible improvements achieved with the use of the weighting function, an abrupt change detection is considered. Given a nominal data batch $\tau^{0} \in \mathcal{Y}_{r}^{0}$, the detection problem is to decide whether a test batch $\boldsymbol{\tau}_{k}$ belongs to $\mathcal{Y}_{r}^{0}$ or $\mathcal{Y}_{r}^{f}$ based on the test quantity $q(k)=\operatorname{KL}\left(\widehat{p}^{0}, \widehat{p}_{k}\right)$ and where the sets $\mathcal{Y}_{r}^{0}$ and $\mathcal{Y}_{r}^{f}$ are given by (19). This corresponds to the following hypotheses

$$
\mathcal{H}^{0}: \tau_{k} \in \mathcal{Y}_{r}^{0}, \quad \mathcal{H}^{1}: \tau_{k} \in \mathcal{Y}_{r}^{f}
$$

where $\mathcal{H}^{0}$ indicates that no wear fault is present, with $\omega=0$, and $\mathcal{H}^{1}$ indicates presence of a wear fault of size $\omega_{c}$. For given values of $\omega_{c}, \xi$ and $\Delta_{\xi}$, the testcycle trajectory $\mho$ is simulated to generate data according to (19). The probability densities of the test quantity under each hypothesis are estimated based on 5000 Monte Carlo runs with and without the use of the weighting function. Based on the hypotheses densities', the probability of detection $P_{d}$ is computed according to (5) for a threshold check when the probability of false alarm is $P_{f}=0.01$.

Figure 6a presents the achieved $P_{d}$ as a function of $\Delta_{\xi}$ for the fixed $\omega_{\mathrm{c}}=35$ and $\underline{\xi}=$ $30^{\circ} \mathrm{C}$ with and without the use of the weighting function. Notice that the use of the weighting function considerably improves $P_{d}$ under temperature variations, but for too large $\Delta_{\xi}$ it becomes difficult to distinguish the effects. A similar study is performed to illustrate how $\omega_{c}$ affects the performance. Figure $6 \mathrm{~b}$ presents $P_{d}$ as a function of $\omega_{\mathrm{c}}$ for the fixed $\Delta_{\xi}=25^{\circ} \mathrm{C}$ and $\underline{\xi}=30^{\circ} \mathrm{C}$. The improvements achieved using the weighted data are clear.

\section{Gearbox Monitoring based on Vibration Data}

In this application, vibration data collected from the gearbox test rig described in Fan and Zuo (2006); Halim et al. (2008) are considered. The test rig is com- 


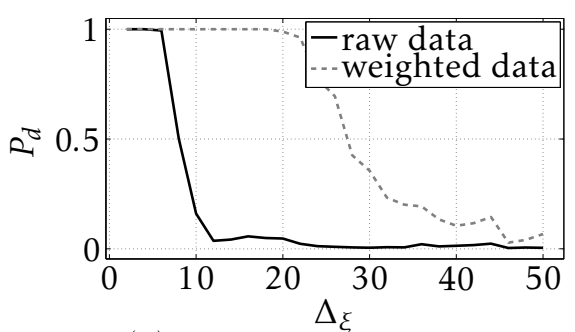

(a) Temperature variations.

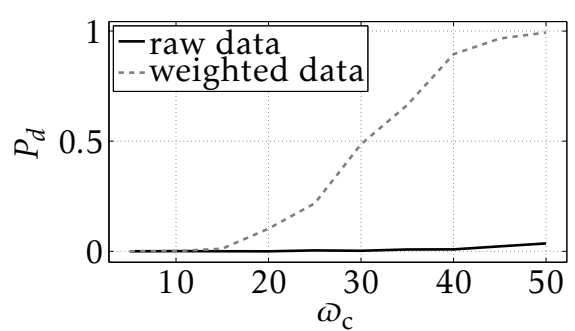

(b) Fault size.

Figure 6: Probability of detection $P_{d}$ when $P_{f}=0.01$ for an abrupt fault with $\omega_{c}=35$ as a function of temperature variations $\Delta_{\xi}$ (a) and as function of the wear change size $\omega_{c}$ for $\Delta_{\xi}=25^{\circ} \mathrm{C}(\mathrm{b})$. Notice the considerable improvements when using the weighted data.

posed of a motor coupled to a gearbox with three shafts and four spur gears. It is possible to study the effects of different types of gear faults in the rig by replacing healthy gears with damaged ones. Four different behavioral modes are considered:

- $m=0$ : healthy gears are used,

- $m=1$ : a gear at the input shaft is damaged,

- $m=2$ : a gear at the output shaft is damaged,

- $m=3$ : a gear at the input and a gear at the output shafts are damaged.

Fault detection approaches for this problem have been proposed in Fan and Zuo (2006) with the use of Hilbert and Wavelet packet transform and in Halim et al. (2008) with a combination of Wavelet transform and time domain averaging. In these approaches, the data are transformed to a time-frequency domain where different faults can be distinguished based on inspection of the transformed data. The distribution domain approach proposed here is considered as an alternative for diagnostics.

Data collected from an accelerometer placed close to the output shaft are considered for the analysis. All data collection was performed under constant load and speed conditions in all settings, ensuring a repetitive behavior. For each different condition, $8 \times 1024$ samples are available with data sampled at $2.56 \mathrm{KHz}$. The datasets are divided in $K=8$ batches with $N=1024$ samples to form $Y_{K}^{m}$ for each mode $m$. Figure 7 a shows the fist data batch $y_{1}^{m}$ for each mode $m$, notice that it is difficult to distinguish differences in the data sequences. In Figure $7 \mathrm{~b}$, the density estimates $\widehat{p}_{k}^{m}(y)$ for every batch in every mode are displayed. Notice the smaller variability of the distribution estimates within each mode compared to the variability found between modes.

To evaluate the detection performance, $q(k)=\operatorname{KL}\left(\widehat{p}^{0}, \widehat{p}_{k}\right)$ is computed for every possible pair $y^{0}$ and $y^{m}$ from $Y_{K}^{0}$ and $Y_{K}^{m}$ respectively for $m \in\{0,1,2,3\}$. Since the distances are computed in pairs, this gives 28 samples from the nominal set 


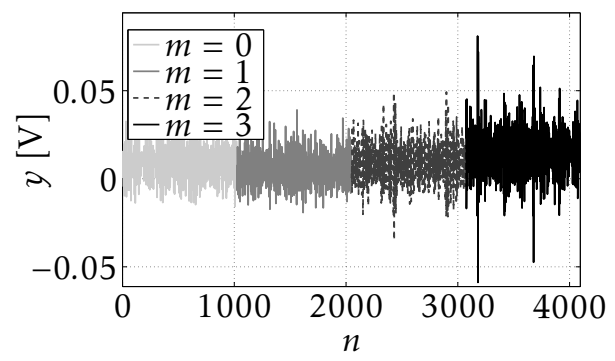

(a) Data sequences.

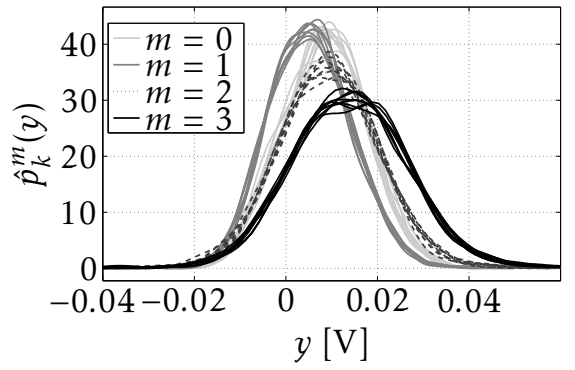

(b) Density estimates.

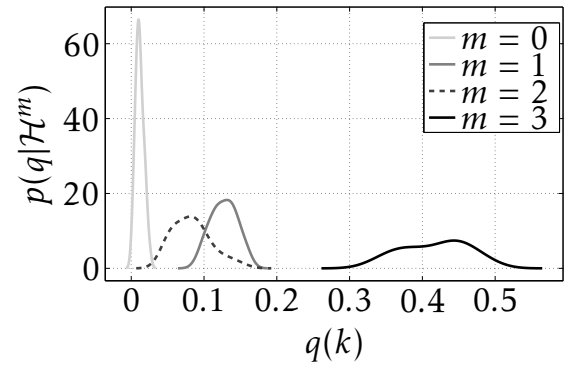

(c) Test quantity behavior.

Figure 7: Diagnostics of a gearbox based on vibration data. A total of four different modes $m$ are possible. An example of data sequence from each mode is shown in (a). The data behavior in the distribution domain is shown in (b). The statistical behavior of the test quantity given by $K L\left(\widehat{p}^{0}, \widehat{p}_{k}\right)$ is shown for $y_{k} \in \mathcal{H}^{m}$ in (c). Notice that, despite the densities in (b) being similar, the test quantity clearly indicates the presence of a change in (c). 
$\mathcal{Q}^{0,0}$ ( $K=8$ combined two by two) and $K^{2}=64$ samples from the sets $\mathcal{Q}^{0, m}$ for $m \in\{1,2,3\}$. The hypotheses densities $p\left(q \mid \mathcal{H}^{m}\right)$ are estimated based on these samples and are shown in Figure $7 \mathrm{c}$. Notice the clear separation between the null hypothesis density, $p\left(q \mid \mathcal{H}^{0}\right)$, from the alternatives. For $P_{f}=0.01, P_{d}$ is computed according to (5) for a threshold check when deciding between $\mathcal{H}^{0}$ and $\mathcal{H}^{m}$ for $m \in\{1,2,3\}$; the achieved values are $1,0.991$ and 1 respectively. To illustrate the approach to isolation as given in (6), the first data batch in each mode, $y_{1}^{m}$, is considered available. The isolation criterion given by

$$
m^{*}=\arg \min _{m \in\{0,1,2,3\}} \operatorname{KL}\left(\widehat{p}_{1}^{m}, \widehat{p}_{k}\right)
$$

chooses the correct mode for all $(K-1) \times 4$ remaining data batches.

\section{Conclusions and Future Work}

The suggested framework considers the monitoring of changes in the distribution of data batches. Because no prior knowledge is assumed about the data behavior, nonparametric kernel density estimates are used which give great flexibility, are simple to implement and have an inherent smoothing behavior. The validity of the framework and methods were illustrated with promising results on real case studies and simulations for gearbox monitoring in robotics and rotating machines. An important advantage of the framework presented is that no model or expert knowledge of the system are required. Furthermore, it gives an alternative for systems where faults affect the data amplitude but where stationary, periodic or linear behaviors are difficult or not possible, as in the robotics application.

In case sets of labeled data batches are available rather than a single one, the generation of test quantities can be performed by considering the distance between a test batch and the labeled sets. When the sets of labeled data batches contain data from broad operations of the system, it is likely that using this simple extension will result in test quantities that present better performance compared to those generated based on a single labeled data batch.

The determination of a change in data collected under repetitive conditions simplifies the diagnostics problem considerably. However, it might not possible to ensure the same repetitive behavior of the system. This is the case, for example, in the industrial robotics application where trajectories are normally only repeated trough a certain period, depending on a manufacturing plan. It is thus relevant to study approaches to handle systems with a varying repetitive behavior. The effects of different kernel functions for the KDE, choice of bandwidth parameter and use of different distances between densities are also important.

\section{Acknowledgments}

The authors would like to thank Professor Sirish L. Shah from the University of Alberta, Canada, for sharing the data used in the studies of Section 5. 


\section{Bibliography}

R. Aguilar-Lopez and R. Martinez-Guerra. Robust state estimation for repetitive operating mode process: Application to sequencing batch reactors. Chemical Engineering Journal, 126(2):155-161, 2007.

M. Basseville. Distance measures for signal processing and pattern recognition. Signal Processing, 18(4):349-369, 1989.

C. M. Bishop. Pattern Recognition and Machine Learning. Springer, New York, USA, 1st edition, 2006.

A. C. Bittencourt and P. Axelsson. Modeling and experiment design for identification of wear in a robot joint under load and temperature uncertainties based on friction data. IEEE/ASME Transactions on Mechatronics, 19(5):1694-1706, October 2014.

A. C. Bittencourt and S. Gunnarsson. Static friction in a robot joint- Modeling and identification of load and temperature effects. Journal of Dynamic Systems, Measurement, and Control, 134(5), July 2012.

A. C. Bittencourt, K. Saarinen, and S. Sander-Tavallaey. A data-driven method for monitoring systems that operate repetitively - applications to wear monitoring in an industrial robot joint. In Proceedings of the 8th IFAC SAFEPROCESS, volume 8, Mexico City, Mexico, 2012.

A. W. Bowman and A. Azzalini. Applied Smoothing Techniques for Data Analysis: The Kernel Approach with S-Plus Illustrations (Oxford Statistical Science Series). Oxford University Press, USA, November 1997.

D. Brambilla, L. Capisani, A. Ferrara, and P. Pisu. Fault detection for robot manipulators via second-order sliding modes. IEEE Transactions on Industrial Electronics, 55(11):3954-3963, November 2008.

F. Caccavale, P. Cilibrizzi, F. Pierri, and L. Villani. Actuators fault diagnosis for robot manipulators with uncertain model. Control Engineering Practice, 17(1): 146-157, 2009.

T. Cacoullos. Estimation of a multivariate density. Annals of the Institute of Statistical Mathematics, 18:179-189, 1966.

A. De Luca and R. Mattone. Actuator failure detection and isolation using generalized momenta. In Proceedings of the 2003 IEEE International Conference on Robotics and Automation (ICRA), volume 1, pages 634-639, Taipei, Taiwan, September 2003.

C. W. de Silva. Vibration Monitoring, Testing and Instrumentation. CRC Press, April 2007.

S. X. Ding, S. Yin, Y. Wang, Y. Wang, Y. Yang, and B. Ni. Data-driven design of observers and its applications. In Proceedings of the 18th IFAC World Congress, Milan, Italy, 2011. 
W. E. Dixon, I. D. Walker, D. M. Dawson, and J. P. Hartranft. Fault detection for robot manipulators with parametric uncertainty: A prediction-error-based approach. IEEE Transactions on Robotics and Automation, 16(6):3628-3634, 2000.

J. Dong, M. Verhaegen, and F. Gustafsson. Robust fault detection with statistical uncertainty in identified parameters. IEEE Transactions on Signal Processing, 60(10):5064-5076, 2012.

R. Durrett. Probability: Theory and examples. Cambridge University Press, August 2010.

I. Eski, S. Erkaya, S. Savas, and S. Yildirim. Fault detection on robot manipulators using artificial neural networks. Robotics and Computer-Integrated Manufacturing, 27(1):115-123, July 2011.

X. Fan and M. J. Zuo. Gearbox fault detection using Hilbert and Wavelet packet transform. Mechanical Systems and Signal Processing, 20(4):966-982, 2006.

V. Filaretov, M. Vukobratovic, and A. Zhirabok. Observer-based fault diagnosis in manipulation robots. Mechatronics, 9(8):929-939, 1999.

B. Freyermuth. An approach to model based fault diagnosis of industrial robots. In Proceedings of the 1991 IEEE International Conference on Robotics and Automation, volume 2, pages 1350-1356, April 1991.

S. C. Guo, M. H. Yang, Z. R. Xing, Y. Li, and J. Q. Qiu. Actuator fault detection and isolation for robot manipulators with the adaptive observer. Advanced Materials Research, 482 - 484(8):529-532, 2012.

E. B. Halim, M. S. Choudhury, S. L. Shah, and M. J. Zuo. Time domain averaging across all scales: A novel method for detection of gearbox faults. Mechanical Systems and Signal Processing, 22(2):261-278, 2008.

R. Isermann. Fault-Diagnosis Systems - An Introduction from Fault Detection to Fault Tolerance. Springer, 1st edition, 2006.

R. Isermann. Fault-diagnosis applications - model-based condition monitoring: actuators, drives, machinery, plants, sensors, and fault-tolerant systems. Springer, New York, 2011.

M. Jones and D. Henderson. Maximum likelihood kernel density estimation: On the potential of convolution sieves. Computational Statistics \& Data Analysis, 53(10):3726-3733, 2009.

X. Li and K. Zhou. A time domain approach to robust fault detection of linear time-varying systems. Automatica, 45(1):94-102, 2009.

L. Ljung. System Identification: Theory for the User. Prentice Hall PTR, 2nd edition, December 1999.

L. Marton and F. van der Linden. Temperature dependent friction estimation: 
Application to lubricant health monitoring. Mechatronics, 22(8):1078-1084, 2012.

M. McIntyre, W. Dixon, D. Dawson, and I. Walker. Fault identification for robot manipulators. IEEE Transactions on Robotics, 21(5):1028-1034, October 2005.

S. Moberg, J. Öhr, and S. Gunnarsson. A benchmark problem for robust control of a multivariable nonlinear flexible manipulator. In Proceedings of the 17th IFAC World Congress, Seoul, South Korea, March 2008.

E. Olsson, P. Funk, and N. Xiong. Fault diagnosis in industry using sensor readings and case-based reasoning. Journal of Intelligent \& Fuzzy Systems, 15: 41-46, December 2004.

E. Parzen. On estimation of a probability density function and mode. The Annals of Mathematical Statistics, 33(3):1065-1076, 1962.

M. D. Reid and R. C. Williamson. Information, divergence and risk for binary experiments. Journal of Machine Learning Research, 12:731-817, 2011.

E. Rogers, K. Galkowski, and D. H. Owens. Control systems theory and applications for linear repetitive processes. Springer, 2007.

E. Rogers, K. Galkowski, and D. H. Owens. Two decades of research on linear repetitive processes part i: Theory. In Proceedings of the 8th International Workshop on Multidimensional Systems (nDS), Erlanger, Germany, 2013a.

E. Rogers, K. Galkowski, W. Paszke, and D. H. Owens. Two decades of research on linear repetitive processes part ii: Applications. In Proceedings of the 8th International Workshop on Multidimensional Systems (nDS), Erlanger, Germany, 2013b.

S. Sander-Tavallaey and K. Saarinen. Backlash identification in transmission unit. In Proceedings of the 2009 IEEE Control Applications \& Intelligent Control, pages 1325-1331, Singapore, Singapore, July 2009.

B. Sulikowski, K. Gałkowski, E. Rogers, and D. H. Owens. Output feedback control of discrete linear repetitive processes. Automatica, 40(12):2167-2173, 2004.

J. I. Taylor. The Vibration Analysis Handbook. Vibration Consultants, February 1994.

H. L. Van Trees. Detection, Estimation and Modulation Theory, Part I. Wiley, New York, 1st edition, 2001.

Y. Wang, G. Ma, S. X. Ding, and C. Li. Subspace aided data-driven design of robust fault detection and isolation systems. Automatica, 47(11):2474-2480, 2011.

L. Wu, X. Su, and P. Shi. Mixed $H_{2} / H_{\infty}$ approach to fault detection of discrete linear repetitive processes. Journal of the Franklin Institute, 348(2):393-414, 2011. 
S. Yin, S. X. Ding, A. H. Abandan Sari, and H. Hao. Data-driven monitoring for stochastic systems and its application on batch process. International Journal of Systems Science, 44(7):1366-1376, 2013. 


\section{Paper D}

\section{Simulation based Evaluation of Fault Detection Algorithms with Applications to Wear Diagnosis in Manipulators}

Authors:

Andreas Samuelsson, André Carvalho Bittencourt, Kari Saarinen, Shiva Sander-Tavallaey, Mikael Norrlöf , Hans Andersson and Svante Gunnarsson.

Edited version of the paper:

A. Samuelsson, A. C. Bittencourt, K. Saarinen, H. Andersson, S. S. Tavallaey, M. Norrlöf, and S. Gunnarsson. Simulation based evaluation of fault detection algorithms with applications to wear diagnosis in manipulators. In Proceedings of the 19th IFAC World Congress, Cape Town, South Africa, 2014. 



\title{
Simulation based Evaluation of Fault Detection Algorithms with Applications to Wear Diagnosis in Manipulators
}

\author{
Andreas Samuelsson ${ }^{* *}$, André Carvalho Bittencourt* Kari Saarinen ${ }^{* *}$, Shiva \\ Sander-Tavallaey $^{* *}$, Mikael Norrlöf ${ }^{* *}$, Hans Andersson ${ }^{* *}$ and \\ Svante Gunnarsson* \\ *Dept. of Electrical Engineering, \\ ${ }^{* *} \mathrm{ABB} \mathrm{AB}$, \\ Linköping University, \\ Västerås, Sweden \\ SE-581 83 Linköping, Sweden
}

\begin{abstract}
Fault detection algorithms (FDAs) process data to generate a test quantity. Test quantities are used to determine the presence of faults in a monitored system, despite disturbances. Because only limited knowledge of the system can be embedded in an FDA, it is important to evaluate it in scenarios of practical relevance. In this paper, simulation based approaches are proposed in an attempt to determine: $i$ ) which disturbances affect the output of an FDA the most; ii) how to compare the performance of different FDAs; and iii) which combinations of fault change size and disturbances variations are allowed to achieve satisfactory performance. The ideas presented are inspired by the literature of design of experiments, surrogate models, sensitivity analysis and change detection. The approaches are illustrated for the problem of wear diagnosis in manipulators where three FDAs are considered. The application study reveals that disturbances caused by variations in temperature and payload mass error affect the FDAs the most. It is also shown how the size of these disturbances delimits the capacity of an FDA to relate to wear changes. Further comparison of the FDAs reveal which performs "best" in average.
\end{abstract}

\section{Introduction}

Fault detection and fault diagnosis can be used to improve safety, reliability, availability, and maintainability of technical systems (Isermann, 2006). In fault detection, observations from the system, e.g., data, are processed and compared to available knowledge of the system to generate symptoms. Symptoms are a partial diagnosis of the system, i.e., a statement about which states of the system could possibly explain the current observations. The diagnosis of complex systems typ- 


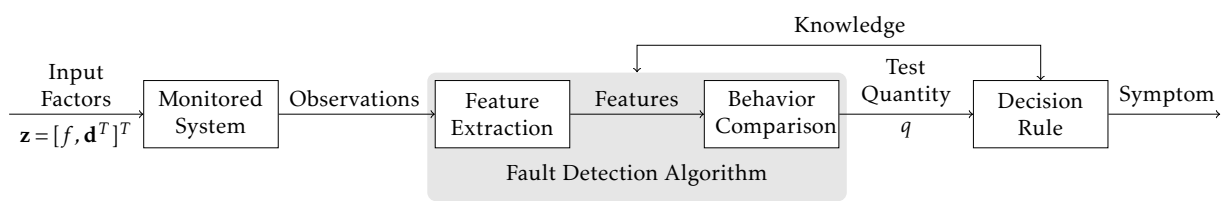

Figure 1: Overview of a fault detection scheme. The monitored system is affected by input factors and generates observations. Features are extracted from the observations which are compared against reference (known) behaviors of the features to generate test quantities. A decision rule determines which behaviors better explain the observations, i.e., it generates a symptom.

ically makes use of several fault detection methods, each containing partial information of the system. In fault diagnosis, the different symptoms are processed to generate a statement of the state (condition) consistent to all observations and knowledge embedded in the diagnosis solution.

While increasing the amount of symptoms used for fault diagnosis may increase the quality of the diagnosis process, it is clear that the accuracy of the symptoms is crucial. Design and evaluation of fault detection methods are therefore important. Figure 1 shows an overall scheme of a fault detection scheme. The monitored system is affected by input factors which are relevant for the diagnosis, e.g., faults and disturbances, and generates observations. The observations are processed to extract relevant features that can describe the status of the system (e.g., parameters, residuals, signal spectra). The behavior of the features are then compared to (known) reference behaviors (e.g., based on distances) to generate a test quantity. The combined tasks of feature extraction and behavior comparison is denoted fault detection algorithm (FDA). Finally, a decision rule (e.g., a threshold check or a statistical test) is used to accept or reject the reference behaviors that the test quantity can explain, i.e., it generates a symptom.

\subsection{Problem description and motivation}

The accuracy of the symptoms generated by the fault detection is determined by the ability of the test quantity generated by the FDA to relate to changes in the system behavior. It is thus natural to evaluate fault detection methods based on the test quantities alone, independent of the decision rule used. This is for instance in line with the theory of statistical hypothesis testing, when an optimal test is given by the likelihood ratio test and is determined only by the statistical behavior of the test quantity, see, e.g., Basseville and Nikiforov (1993).

A test quantity, denoted $q$, measures deviations from one or more reference behaviors. The reference behaviors are associated to states of the system, e.g., healthy or faulty. In this work, the focus is on the analysis of a single fault, denoted $f$. Rather than considering test quantities which are time sequences, e.g., model residuals, the focus is restricted to batch fault detection algorithms, which produce a scalar 
$q$ for an entire data batch. Batch methods are common for signal/data-driven approaches and parameter estimation, but similar ideas could be used also for time sequences by summarizing the sequence to a scalar, e.g., by considering steadystate values or some norm.

In practice, the data input to fault detection (and thus $q$ ) are not only affected by the fault $f$ but by a collection of $n$ factors $z$. In this paper, the input factors considered in the examples are a composition of the fault $f$ and sources of disturbances $\boldsymbol{d}$, i.e., $\boldsymbol{z}=\left[f, \boldsymbol{d}^{T}\right]^{T}$, where $\boldsymbol{d}=\left[d_{1}, \cdots, d_{i}, \cdots, d_{n-1}\right]^{T}$. The disturbances, $\boldsymbol{d}$, may cause undesired variations to $q$, deteriorating its capacity to distinguish changes in $f$ and thus complicating a decision. Under specified conditions and assumptions, optimality of FDAs might be possible, see, e.g., Liu and Zhou (2008); Li and Zhou (2009); Frank and Ding (1997); Wei and Verhaegen (2011), and it may be possible to compare different schemes (Isermann, 1994, 2006). However, since only partial knowledge of the system can be embedded in any FDA, it is important to evaluate it in scenarios which are relevant for its practical use. From a practical perspective, given a complex system and candidate FDAs, the following questions are of relevance:

Q-1 Which factors in $\boldsymbol{d}$ affect $q$ the most? And should therefore be given more relevance for further development of the FDA.

Q-2 How can test quantities generated from different FDAs be compared and evaluated against each other to enable selection of the "best" FDAs?

Q-3 What is the effective scope of an FDA? That is, for what region in the $z$ space is the ability of $q$ to relate to $f$ satisfactory?

Notice that the focus is not on properties of a particular FDA but to define approaches to evaluate and compare any FDA.

These questions can be addressed at different levels of closeness to the real application. Level 0 corresponds to the ideal case where the FDAs are evaluated with operational data. This is particularly difficult since it may take extremely long times for faults to appear. To overcome this, data can be collected from experiments performed in a lab, where faults and disturbances are induced, corresponding to Level 1 studies. Even at Level 1, an extensive evaluation is often inviable due to the extreme costs and time required. Furthermore, it is often the case that all (or parts of) the factors $z$ are unmeasurable and therefore a complete analysis based on real data is difficult. At Level 2, data are generated based on simulations of the monitored system, which is a more viable alternative. The simulation study must, on the other hand, be designed carefully so that it is representative of practical scenarios.

\subsection{Main contributions and outline}

In this paper, ideas inspired by the literature of design of experiments, surrogate models, sensitivity analysis and change detection are presented to address these questions based on simulation studies. Even in simulation studies, an extensive analysis of the effects of $z$ to $q$ may exhaust the computational resources and time available. An important idea considered here is to bypass the need for 
simulation/experimental data using a surrogate (or meta) model. Different types of surrogate models are possible, e.g., based on neural networks and Gaussian processes. For its simplicity and tractability, the surrogate models considered here will take the form of a linear regression,

$$
q=\phi(z)^{T} \boldsymbol{\theta}+\epsilon, \quad \phi(\cdot): \mathbb{R}^{n} \mapsto \mathbb{R}^{n_{\theta}}, \quad q, \epsilon \in \mathbb{R}
$$

where the regressors function $\phi(\cdot)$ makes a direct map from $z$ to $q$ through the regression coefficients $\theta$ and $\epsilon$ is an additive uncertainty term. The surrogate model incorporates both the monitored system and FDA. Studies based on surrogate models are denoted as Level 3. In such approach, the choice of factors $z$ and regressors, the identification of $\theta$ and model validation are important and are subject of study in the field known as design of experiments (DOE) which is briefly described in Section 2.

An answer to Q-1 is presented in Section 3, where the coefficients $\theta$ of the regression models are studied using sensitivity analysis to determine which factors in $\boldsymbol{d}$ affect $q$ the most. A main advantage with the use of surrogate models is that Monte Carlo (MC) simulations can be performed efficiently. MC runs are used in Section 4 to evaluate a measure of average effects of changes in $q$ by $f$ which is used to address Q-2. In Section 5, a measure of satisfactory performance is suggested which is evaluated with MC runs under various combinations of $z$ in an attempt to answer Q-3. In Section 6, the ideas are illustrated for the evaluation of methods used for wear diagnosis in industrial robots. Relevant characteristics of the problem and methods are revealed from the study. Concluding remarks are given in Section 7.

\section{Design of Experiments}

Design of experiments (DOE) can be applied to any system where the experimenter has control over the input variables, or input factors, and that the output can be measured (Kleijnen et al., 2005). Here, the object of study is an FDA applied in combination to a monitored system. The input factors are, e.g., faults and disturbances that may affect the behavior of the monitored system, and the output is the test quantity $q$. The next sections are organized to give an introduction to the field, for more details see, e.g., Box et al. (1978); Kleijnen et al. (2005); Sanchez (2006).

\subsection{Choice of input factors}

The first task in designing an experiment is the selection of the input factors $z$ and their possible range of values. Factors can be included according to the objectives of the study, to verify or falsify assumptions about the behavior of the test quantity and to study their relations in detail. The choice of factors should be performed carefully, with the help of experts in the application, since a poor specification may generate misleading results. For the examples presented here, input factors include the fault, $f$, and disturbance factors, $\boldsymbol{d}$, but other types of 
factors could be included such as initial conditions, tuning variables, etc.

Once the factors are chosen, the experimenter must decide their range of values and a discrete set of factor levels that shall be considered in the study. A more detailed study is possible by increasing the number of levels, $m$, in a compromise with the number of experiments required. The factor levels chosen will have an impact on the study and it is therefore important to choose levels which are extreme but not impossible for realistic situations. Two representations of factor levels are typically used:

- natural levels are the values for the factors that are used in the experiment or simulation;

- coded levels, all factors are normalized to the same scale. Used when identifying the surrogate models.

The normalization of the coded levels is important because otherwise the parameters of the surrogate model would be affected by the scaling of the factors.

\subsection{Surrogate models as linear regressions}

Linear regression models as in (1) are simple, tractable and easy to interpret. For these reasons, they are a popular choice in the DOE literature. A limitation is that they may misrepresent the relations between $z$ and $q$. To circumvent this, more complex model structures, such as neural networks and Gaussian processes could be considered, see, e.g., Oakley and O'Hagan (2004). Compared to linear regressions, more complex models may be less interpretable and tractable which are important characteristics for surrogate models. Easy to interpret models are particularly important for sensitivity analysis, studied in Section 3.

Many different model structures of linear regressions can be considered. From the DOE literature, two structures are commonly used. A main effects model has regressors that are directly dependent on the inputs factors, i.e.,

$$
\begin{aligned}
& \phi(z)^{T}=\left[\begin{array}{ll}
1, & z^{T}
\end{array}\right]=\left[\begin{array}{lllll}
1, & f, & d_{1}, & \cdots, & d_{n-1}
\end{array}\right] \\
& \boldsymbol{\theta}^{T}=\left[b, \quad\left\{\eta_{i}\right\}\right]
\end{aligned}
$$

where $b$ is a bias term and $\eta_{i}$ has indices $i \in\{0, \cdots, n-1\}$. Since this is a simple model, it may not be a realistic representation of the system. A second-order model extends the main-effects model with interaction (cross) and quadratic terms as

$$
\begin{aligned}
\phi(z)^{T} & =\left[\begin{array}{lll}
1, & z^{T}, & \operatorname{svec}\left(z z^{T}\right)^{T}
\end{array}\right] \\
\boldsymbol{\theta}^{T} & =\left[\begin{array}{llll}
b, & \left\{\eta_{i}\right\}, & \left\{\eta_{i j}\right\}, & \left\{\eta_{i i}\right\}
\end{array}\right]
\end{aligned}
$$

where $\operatorname{svec}(\cdot)$ maps a symmetric matrix of size $N$ to a vector of length $N(N+1) / 2$ and $i, j \in\{0,1, \cdots n-1\}$ with $i>j$. A second order model can capture more complex relations between the factors than a main effects model. However, since each factor is included in several terms, it is more difficult to analyze the effects of different factors to $q$. Notice that the models can be extended further with any type of relation between the factors. 


\subsection{Identification}

Consider that $N$ experiments are performed with inputs

$$
Z_{N}=\left[z_{1}, \cdots, z_{N}\right]^{T} \in \mathbb{R}^{N \times n}
$$

and outputs $q \in \mathbb{R}^{N}$. Given that the test quantities can be described by (1), the resulting model is $\boldsymbol{q}=\Phi\left(Z_{N}\right) \boldsymbol{\theta}+\boldsymbol{\epsilon}$ with $\Phi\left(Z_{N}\right) \triangleq\left[\phi\left(z_{1}\right), \cdots, \phi\left(z_{N}\right)\right]^{T}$. To find the coefficients $\theta$, a least-squares error criterion gives

$$
\begin{aligned}
\widehat{\boldsymbol{\theta}} & =\arg \min _{\boldsymbol{\theta}}\left\|\boldsymbol{q}-\Phi\left(Z_{N}\right) \boldsymbol{\theta}\right\|_{2}^{2} \\
& =R_{N}\left(Z_{N}\right)^{-1} \Phi\left(Z_{N}\right)^{T} \boldsymbol{q}, \\
R_{N}\left(Z_{N}\right) & \triangleq \Phi\left(Z_{N}\right)^{T} \Phi\left(Z_{N}\right) .
\end{aligned}
$$

The estimate is unbiased and consistent in case the errors are uncorrelated with the regressors and has finite variance (see Ljung (1999)). For $N$ observations, an estimate of the covariance of $\widehat{\boldsymbol{\theta}}$ is found through the expression (see Ljung (1999))

$$
\begin{aligned}
& \widehat{P_{\widehat{\theta}}}=\widehat{\gamma} R_{N}\left(Z_{N}\right)^{-1} \\
& \widehat{\gamma} \triangleq \frac{1}{N-n-1}\left\|\boldsymbol{q}-\Phi\left(Z_{N}\right) \widehat{\boldsymbol{\theta}}\right\|_{2}^{2},
\end{aligned}
$$

where $\widehat{\gamma}$ is an estimate of the error variance. Notice again that the coded levels should be used when identifying the regression coefficients. Otherwise, the scaling of the variables will hinder some of the analysis presented further.

\subsection{Design matrix}

A design matrix represents the user choice of simulation experiments to be performed. Typically, the columns correspond to the factor levels and rows are design points, i.e., a specific choice of the coded levels $z$. Using the previously introduced notation, a design matrix corresponds to a specific choice of $Z_{N}$ in (4). Some designs are briefly described below.

Full Factorial Design, $N=m^{n}$. In this design, the $m$ possible levels for the $n$ factors are combined exhaustively, so the design matrix has $N=m^{n}$ design points. The number of experiments increases rapidly with $n$, e.g., for $m=5, n=6$, a total of $N=15625$ experiments are needed. This design is rather conservative and more efficient designs are available.

Fractional Factorial Design, $N=m^{n-f}$. To reduce the complexity of a full factorial design, it is possible to carefully select subsets of a full design, where $m^{f}$ is the fraction removed.

Central Composite Design. These designs are useful to efficiently identify higher order models (Box and Wilson, 1951). The design is usually based on a full or fractional factorial. The base factorial design is then extended by adding a center point and two "star points" for each factor. This design is illustrated by the following example. 
Example. A central composite design for $n=3$ factors based on $2^{3}$ full factorial design (black), a center point and star points at the faces (gray).

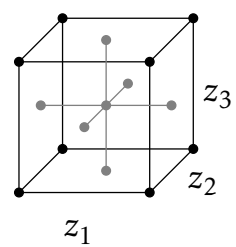

Optimal Designs. By noting that the covariance of $\widehat{\boldsymbol{\theta}}$ given in (7) depends on $R_{N}\left(Z_{N}\right)$, it is natural to define minimum-variance designs, that minimize some measure of $R_{N}\left(Z_{N}\right)$. Different types of optimal designs are possible depending on the measure used, a D-optimal design will minimize the determinant of $R_{N}\left(Z_{N}\right)$ while an A-optimal design will minimize its trace. For more on optimal designs, see, e.g., Fedorov (1972); Atkinson et al. (2007).

\subsection{Design parameters}

The validity of surrogate models is limited since the behavior of the test quantities can differ considerably depending on different variables. For example, it should not be expected that the same surrogate model can be used to describe test quantities from different FDAs or when applied to different monitored systems. The settings that determine the validity of the surrogate models are called design parameters and one surrogate model should be identified for each different combination of design parameters considered in the study.

\subsection{Model validation}

The analysis performed in this paper are based on surrogate models and it is therefore important to validate them. Model validation is used to assess whether the model will generalize to input values independent of those used during the model identification. Model validation can be done by cross-validation, where a fresh dataset, denoted $Z_{N_{v}}^{v}$, is used with the sole purpose of validation.

The discrepancies between the model and the system behaviors can be studied through the residuals, $\varepsilon$, which are defined as the differences between the system and model outputs,

$$
\varepsilon=\boldsymbol{q}-\widehat{\boldsymbol{q}}, \quad \widehat{\boldsymbol{q}} \triangleq \Phi\left(Z_{N_{v}}^{v}\right) \widehat{\boldsymbol{\theta}},
$$

where $Z_{N_{v}}^{v}$ represents a validation data set. If the residuals are small, the model is considered valid, otherwise it is invalidated and perhaps a different model structure should be considered.

The model fit (Ljung, 1999) can be used as a criterion to assess the validity of a model. It is defined as

$$
\text { fit }=100\left(1-\frac{\|\varepsilon\|_{2}^{2}}{\|\boldsymbol{q}-\bar{s}\|_{2}^{2}}\right), \quad \bar{s} \triangleq \frac{1}{N_{v}} \sum_{i}^{N_{v}} s_{i} .
$$

The model fit relates to how well the model predicts the output in average. 


\section{Determining Relevant Factors}

An approach to address Q-1 is to study how changes in a factor affect the test quantity $q$. The partial derivatives of the surrogate model with respect to the factors $z$ reveal how the first order properties of $q$ are affected by $z$. This type of study is part of sensitivity analysis (Saltelli et al., 2008). For the main-effects model (2), the partial derivatives are given by

$$
\frac{\partial}{\partial z} q(z)=\frac{\partial}{\partial z} \phi(z)^{T} \boldsymbol{\theta}=\frac{\partial}{\partial z}[1, \quad z]^{T}\left[\begin{array}{ll}
b, & \eta
\end{array}\right]=\eta^{T}
$$

and the first order effects of factor $z_{i}$ to $q$ are given directly by the associated $\eta_{i}$. Since coded (normalized) levels of $z$ are used, an inspection of the magnitude of the elements of $\eta$ can be used to find which factors affect $q$ the most.

For more complex models, such as the second-order model (3), the partial derivatives depend not only on $\eta$ but also on the values of $z$ where they are evaluated. Therefore, a direct comparison of the coefficients $\eta$ does not have the same character as for a main effects model. However, for simple regression models, an inspection of $\eta$ can still provide useful insights about the effects of $z$ to $q$.

\subsection{Normalization of coefficients}

For regression models where the fault has a direct term as

$$
\begin{aligned}
\phi(z)^{T} & =\left[\begin{array}{lll}
1, & f, & \cdots
\end{array}\right] \\
\boldsymbol{\theta}^{T} & =\left[\begin{array}{lll}
b, & \eta_{0}, & \{\eta\}_{i>0}
\end{array}\right]
\end{aligned}
$$

the coefficient $\eta_{0}$ relates to the direct effect of the fault $f$ to $q$. To facilitate the study and comparison of coefficients, the identified coefficient vector $\widehat{\eta}$ for models of the form (11) can be normalized as

$$
\bar{\eta}=\widehat{\eta} / \widehat{\eta}_{0} \text {. }
$$

In this manner, the normalized coefficients have values relative to the direct effect of the fault $f$. A normalized coefficient with $\left|\bar{\eta}_{i}\right|<1$ would thus mean that $f$ has a direct effect to $q$ which is larger than that caused by the regressor associated with $\bar{\eta}_{i}$. The case $\left|\bar{\eta}_{i}\right|>1$ is possible but undesirable (unless $\bar{\eta}_{i}$ also relates to $f$ ). Notice that $\bar{\eta}_{0}=1$.

\subsection{Group analysis}

The normalized coefficients in (12) can be grouped together over a subset of the design parameters to investigate different aspects of the problem. For example, consider a problem with two design parameters corresponding to the FDA used and the monitored system. Groups formed for each FDA over all monitored systems would allow for an overall comparison of the FDAs sensitivity. On the other hand, groups formed for each monitored system could be used to reveal which systems are more difficult to perform fault detection, independent of the FDA chosen. 
Suppose there are $K$ groups, where each $k$ th group has $N_{k}$ regression models. The following matrix can be formed for the $k$ th group

$$
H_{k}=\left[\begin{array}{lll}
\bar{\eta}_{1}, & \cdots, \bar{\eta}_{N_{k}}
\end{array}\right]^{T} \in \mathbb{R}^{N_{k} \times N_{\eta}} .
$$

Each group can be analyzed using box plots for each column of $H_{k}$, i.e., each type of coefficient. This type of analysis is illustrated further in Section 6.2.

\section{Comparing Fault Detection Algorithms}

A simple approach to address Q-2 is to analyze the average effects a change in $f$ gives to $q$ when random changes of the disturbance factors $d$ are present. To proceed, a change is defined in terms of hypotheses in Section 4.1 and a measure of average change to the test quantity is defined in Section 4.2.

\subsection{Two hypotheses}

The performance of a test quantity is associated to how well it can be used to relate the presence of a change from nominal in $f$, irrespective of variations in the disturbances $\boldsymbol{d}$. Given a test quantity $q$, two hypotheses are considered. The null hypothesis, $\mathcal{H}^{0}$, represents the case where $q$ is collected when $f$ is nominal and the alternative hypothesis, $\mathcal{H}^{1}$, states that an abnormal change in $f$ is present. These hypotheses can be described by the particular choices of input factors

$$
\begin{array}{lll}
\mathcal{H}_{0}: & f=f^{0}, & \boldsymbol{d} \sim p(\boldsymbol{d}), \\
\mathcal{H}_{1}: & f=f^{0}+\Delta, & \boldsymbol{d} \sim p(\boldsymbol{d}),
\end{array}
$$

where $f^{0}$ is the nominal value of $f, \Delta$ is the fault change size and $p(\boldsymbol{d})$ is a distribution for the (considered random) disturbance factors $\boldsymbol{d}$. Test quantities collected under the different hypotheses are denoted as $q \mid \mathcal{H}^{0}$ and $q \mid \mathcal{H}^{1}$.

\subsection{A measure of average effects}

Denoting $\mu^{i}, \sigma^{i}$ the mean and standard deviation of $q \mid \mathcal{H}^{i}$, for the hypotheses given in (14) the signal to noise ratio (SNR) is defined as

$$
\mathrm{SNR} \triangleq \frac{\mu^{1}-\mu^{0}}{\sigma^{1}},
$$

and relates to the average effects a change of size $\Delta$ in $f$ causes to the test quantity in relation to effects of random variations in $\boldsymbol{d}$. The larger the SNR value, the easier it will be to distinguish the change in $f$. In order to find the quantities used in the computation of the SNR, Monte Carlo runs can be performed for different realizations of $\boldsymbol{d}$ until enough samples of $q \mid \mathcal{H}^{0}$ and $q \mid \mathcal{H}^{1}$ are collected for the estimation of $\mu^{0}, \mu^{1}, \sigma^{1}$. Here, the use of surrogate models instead of experiments allows for efficient MC runs, and the quantities can be found accurately and in short time. Notice that, in some cases, the SNR can be found analytically, e.g., when a main-effects model is used and $p(\boldsymbol{d})$ is the Gaussian distribution. 


\subsection{Group analysis}

In a similar manner as discussed in Section 3.2, the SNRs can be grouped over subsets of design parameters to asses different aspects of the problem. Notice that the SNRs are already normalized quantities. The use of SNRs for comparison of FDAs is illustrated in Section 6.3.

\section{Determining the Effective Scope}

To address Q-3, a measure of satisfactory performance of a test quantity should be defined. Once the performance criterion is defined, it is possible to investigate what region in the $z$ space is the criterion fulfilled. That is, the effective scope of the test quantity can be found.

\subsection{A measure of satisfactory performance}

The behavior of a test quantity should be such that it allows for an accurate decision of whether a change in $f$ is present or not. Presence or absence of a change in $f$ can be described by the two hypotheses in (14). The SNR, introduced in Section 4 , can be used as a performance measure since it relates to how accurate a decision can be made. The performance of an FDA can be considered satisfactory in case the SNR is large enough, e.g., written as

$$
\text { pass }= \begin{cases}1, & \text { if } \mathrm{SNR} \geq \mathrm{SNR}^{\prime}, \\ 0, & \text { otherwise }\end{cases}
$$

where SNR' is a lower limit for the SNR. This criterion is simple to evaluate but since no decision mechanism is defined, it is an indirect measure of performance for the fault detection.

For a given decision rule, the accuracy of the fault detection can be defined in terms of the probabilities of false detection $P_{f}$, i.e., accepting $\mathcal{H}^{1}$ when $\mathcal{H}^{0}$ is true, and correct detection $P_{d}$, i.e., accepting $\mathcal{H}^{1}$ when $\mathcal{H}^{1}$ is true. A natural performance criterion is thus defined according to acceptable levels of $P_{d}$ and $P_{f}$. This can be tested with the function

$$
\text { pass }= \begin{cases}1, & \text { if } P_{f} \leq P_{f}^{\prime} \text { and } P_{d} \geq P_{d}^{\prime}, \\ 0, & \text { otherwise }\end{cases}
$$

where $P_{f}^{\prime}$ and $P_{d}^{\prime}$ are the chosen performance requirements. For a satisfactory performance of the test quantity, low $P_{f}$ and high $P_{d}$ are typically desirable.

The probabilities $P_{f}$ and $P_{d}$ are however dependent on the decision rule used. Different decision rules are possible, see, e.g., Gustafsson (2000). Here, a threshold check is considered since it is one of simplest and is also a common choice. It is defined as

$$
\left\{\text { Choose } \mathcal{H}^{0} \text { if: } s \leq \hbar \text {. Otherwise, choose } \mathcal{H}^{1}\right\} \text {, }
$$


where $\hbar$ is a threshold. For the threshold check (18) with threshold value $\hbar, P_{f}$ and $P_{d}$ can be computed as

$$
P_{f}=\int_{\hbar}^{\infty} p\left(q \mid \mathcal{H}^{0}\right) \mathrm{d} q, \quad P_{d}=\int_{\hbar}^{\infty} p\left(q \mid \mathcal{H}^{1}\right) \mathrm{d} q .
$$

where $p\left(q \mid \mathcal{H}^{0}\right)$ and $p\left(q \mid \mathcal{H}^{1}\right)$ denote the probability densities of $q$ under the different hypotheses. Notice that according to (19), for a fixed $P_{f}$ there is an associated $\hbar$ and thus a $P_{d}$. The criterion (17) can therefore be verified by first finding $\hbar$ for the limiting value $P_{f}^{\prime}$, computing the associated $P_{d}$ and checking whether it is larger than $P_{d}^{\prime}$. The hypotheses densities can be estimated given a large number of observations for $q \mid \mathcal{H}^{0}$ and $q \mid \mathcal{H}^{1}$, which can be achieved efficiently with MC runs using surrogate models.

\subsection{Finding the effective scope}

To find the scope of a test quantity, the chosen criterion for satisfactory performance, e.g., (16) or (17), can be verified for multiple setups of the hypotheses in (14). In order to simplify the analysis, one disturbance factor is varied randomly at a time while the others are kept constant. This setup can be described by the hypotheses

$$
\begin{array}{lll}
\mathcal{H}^{0}: f=f^{0}, & d_{j \neq i}=d_{j}^{\prime}, & d_{i} \sim p(d) \\
\mathcal{H}^{1}: f=f^{0}+\Delta, & d_{j \neq i}=d_{j}^{\prime}, & d_{i} \sim p(d)
\end{array}
$$

i.e., the $i$ th disturbance factor is varied randomly while the remaining are kept constant. By checking the criterion for different choices of $\Delta$ and $p(d)$ in (20), it is possible to gather understanding of the effective scope of the test quantity.

With this purpose, it might be useful to restrict how the distribution $p(d)$ can be varied. Consider for instance that $p(d)$ has zero mean and variance $\sigma^{2}$. By varying $\sigma$, it is then possible to study how much variability of $d_{i}$ is allowed for a satisfactory performance. Considering that $\Delta$ and $\sigma$ can be chosen from the vectors

$$
\Delta=\left[\begin{array}{lll}
\Delta_{1}, & \cdots, & \Delta_{N_{\Delta}}
\end{array}\right]^{T}, \quad \sigma=\left[\begin{array}{lll}
\sigma_{1}, & \cdots, & \sigma_{N_{d}}
\end{array}\right]^{T},
$$

all possible combinations of $\Delta$ and $\sigma$ define a grid of size $N_{\Delta} \times N_{d}$. The satisfactory performance criterion for each pair $(\Delta, \sigma)$ in the grid can be stored in a binary matrix of the same size, denoted scope matrix. Because each entry in a scope matrix relates to whether the performance criterion is achieved, its inspection allows for a straightforward analysis of the scope of a test quantity.

\subsection{Group analysis}

Scope matrices can be found for each regression model. In a similar manner as discussed in Sections 3.2 and 4.3, scope matrices can be grouped over subsets of design parameters. Because each entry in the matrices is either zero or one, the 
information in the group can be summarized by summing over its scope matrices. In this case, the entry values of the resulting group scope matrix will correspond to how many times has successful performance been achieved for the corresponding combination of $\Delta$ and $\sigma$ over the design parameters in the group. This type of analysis is illustrated in Section 6.4 for the robotics application.

\section{Evaluation of Fault Detection Algorithms for Wear Monitoring in Robots}

The framework is illustrated for the problem of wear diagnosis in an industrial robot joint. As empirically shown in Bittencourt and Axelsson (2014) from accelerated wear tests (Level 1 studies), wear in a robot joint can lead to variations of friction. Since the friction torques must be overcome by the motor torques during operation, it is possible to extract information about friction (and wear) from available signals. Friction is however dependent on other factors than wear, such as temperature and load. The effects of temperature are specially difficult since temperature is not measured in typical robot applications. These effects should nevertheless be considered when evaluating different fault detection algorithms.

To simplify the presentation and due to confidentiality issues, the FDAs considered in the study are treated as black-boxes, processing data to generate a test quantity $q$, recall Figure 1. The focus is placed on the evaluation and comparison of the FDAs. The FDAs considered in this study share the following characteristics, which are relevant for the presentation of the paper.

C-1 Process data batches collected from a test-cycle.

C-2 Output a scalar quantity for each data batch.

C-3 Require nominal (wear-free) data.

C-4 Process data for a single axis and should indicate wear changes only for that axis.

C-5 The behavior of a test quantity depends on a combination of FDA, robot, axis and test-cycle.

Data for the study are collected at Level 2, i.e., based on simulation experiments, using an $A B B$ internal simulation tool and the analysis results are achieved at Level 3, with the use of surrogate models. A simplified version of the friction model presented by Bittencourt and Axelsson (2014) is included in the simulation model. The model used to describe friction in the robot joints is given by the static nonlinear function

$$
\begin{aligned}
\tau_{f}(\dot{\varphi}, \xi, \omega)=\left(\theta_{0}+\left(\theta_{1}+\theta_{2} \xi\right) e^{-\left|\frac{\dot{\varphi}}{\theta_{3}+\theta_{4} \xi}\right|}\right. & \left.+\theta_{7} e^{-\left|\frac{\dot{\varphi}}{\theta_{8} \omega}\right|}\right) \operatorname{sign}(\dot{\varphi}) \\
& +\left(\theta_{5}+\theta_{6} \xi+\theta_{9} \omega\right) \dot{\varphi},
\end{aligned}
$$

and relates to the effects of angular speed, $\dot{\varphi}$, temperature (as measured in the joint lubricant), $\xi$, and wear fault, $\omega$, to friction, $\tau_{f}$. The remaining variables $\theta$ are model parameters, see Bittencourt and Axelsson (2014). 
The complete setup for the study includes.

S-1 Three FDAs for wear monitoring, denoted $A, B$ and $C$.

S-2 Two robot simulation models, corresponding to a medium sized robot with max. payload of $10-25 \mathrm{~kg}$ and of a large robot with max. payload of $100-$ $250 \mathrm{~kg}$.

S-3 Wear is studied in the first three axes of these robots.

S-4 A total of six different test-cycles.

The study is aimed at answering questions Q-1 to Q-3 for the robotics application. The next sections define the experiments performed and present the results.

\subsection{Design of experiments}

\section{Input factors}

The following factors are considered relevant and are included in the study.

Wear. According to S-3, wear is introduced in three of the axes. Recalling that the FDAs process data for a single axis (C-4), the wear introduced in that axis will correspond to the fault factor $f$. When wear is present in the other two axes, they may cause variations in $q$ due to coupling effects. Since these variations may complicate fault isolation, they are considered as disturbances, $d_{1}$ and $d_{2}$. The wear $\omega$ in (22) is a dimensionless quantity with values between 0 (no wear) and 100 (a total failure due to wear), see Bittencourt and Axelsson (2014) for details. In this study, it is considered that values in the range $[0,50]$ are of interest. This is because the detection of a partial failure is more interesting for condition-based maintenance since it gives enough time to perform maintenance before a failure.

Temperature. The friction model used given in (22) includes temperature dependencies which will affect the data used for the FDAs. The temperature factor is assigned as $d_{3}$. The temperature range considered is $[30,70]{ }^{\circ} \mathrm{C}$ and is based on a typical temperature behavior for a robot operating in a room with controlled environment temperature. The range copes with variations due to self-heating caused by losses in the joint and changes in the environment temperature.

Point-to-point delay. In point-to-point movements, the robot is required to fulfill a set of criteria in order to guarantee that a certain position was reached before issuing a command to move to the next position. During real-time path execution, the time required for the verification of these criteria may differ, causing variations to the trajectory. This varying "delay" is expected to have an effect on the test quantities and is thus included as a factor, $d_{4}$. The range of values for $d_{4}$ is $[25,75] \mathrm{ms}$ and is based on values found for the robots studied.

Payload mass error. The control system used in the robot relies on the defined payload mass. The closed-loop system (and data) will thus be affected in case there is an error in the defined mass. The payload mass error is assigned as $d_{5}$ and has values in the interval $[-10,10] \%$ relative to the correct mass.

For the study, five levels are considered for each factor, i.e., $m=5$. The levels 
Table 1: Definition of the factor levels used.

\begin{tabular}{lcccccc} 
& \multicolumn{5}{c}{ Coded Levels } & \\
\cline { 2 - 5 } Factor & -2 & -1 & 0 & 1 & 2 & \multirow{5}{*}{ Natural Levels } \\
\cline { 2 - 5 } & 0 & 12.5 & 25 & 37.5 & 50 & - \\
\hline$f, d_{1}, d_{2}$ - wear & 30 & 40 & 50 & 60 & 70 & ${ }^{\circ} \mathrm{C}$ \\
$d_{3}$ - temperature & 25 & 37.5 & 50 & 62.5 & 75 & $\mathrm{~ms}$ \\
$d_{4}$ - point-to-point delay & -10 & -5 & 0 & 5 & 10 & $\%$ \\
$d_{5}$ - payload mass error & -5 & &
\end{tabular}

Table 2: Some entries of the design matrix.

\begin{tabular}{|c|cccccc|r|cccccc|}
\hline Row & $f$ & $d_{1}$ & $d_{2}$ & $d_{3}$ & $d_{4}$ & $d_{5}$ \\
\hline 1 & -1 & -1 & -1 & -1 & -1 & -1 & 41 & 0 & 0 & 0 & 0 & -2 & 0 \\
2 & -1 & -1 & -1 & -1 & 1 & 1 \\
3 & -1 & -1 & -1 & 1 & -1 & 1 & 42 & 0 & 0 & 0 & 0 & 2 & 0 \\
3 & -1 & -1 & -1 & 1 & 1 & -1 & 0 & 0 & 0 & 0 & 0 & -2 \\
4 & -1 & 4 & 0 & 0 & 0 & 0 & 0 & 2 \\
5 & -1 & -1 & 1 & -1 & -1 & 1 & 45 & 0 & 0 & 0 & 0 & 0 & 0 \\
\cline { 1 - 3 }
\end{tabular}

are distributed linearly within the suitable range for the factors. The factor levels used can be seen in Table 1. According to C-3, the test quantities require nominal (wear-free) data which are generated according to the following coded levels,

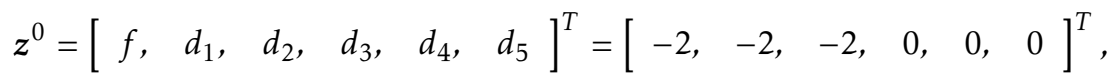

i.e., no wear is present in any of the axes, with temperature at $50^{\circ} \mathrm{C}, 50 \mathrm{~ms}$ of point-to-point delay and no error in payload mass.

\section{Regression models}

Two model structures are considered, a full second-order model as in (3) and a simplified second order model of the form

$$
\begin{aligned}
\varphi(z)^{T} & =\left[\begin{array}{llll}
1, & f, & \boldsymbol{d}^{T}, & \operatorname{svec}\left(\boldsymbol{d} \boldsymbol{d}^{T}\right)^{T}
\end{array}\right] \\
\boldsymbol{\theta}^{T} & =\left[\begin{array}{lllll}
b, & \eta_{0}, & \left\{\eta_{i}\right\}, & \left\{\eta_{i j}\right\}, & \left\{\eta_{i i}\right\}
\end{array}\right]
\end{aligned}
$$

where $i, j \in\{1, \cdots, n\}$ with $i>j$. Notice that there are no cross-terms for the fault $f$ in model structure (24), only for the disturbances $\boldsymbol{d}$. An interpretation of the coefficients for this model is thus simpler compared to the full second-order model.

\section{Design matrix}

A central composite design based on a $2^{n-1}$ fractional factorial design with one center point and star points at $[2,-2]$ is considered, requiring a total of $N=45$ experiments. Parts of the values for the design matrix are seen in Table 2. 
Table 3: Factor levels used for validation.

\begin{tabular}{lcccccc} 
Factor & \multicolumn{5}{c}{ Natural Levels } & Unit \\
\hline$f, d_{1}, d_{2}$ - wear & 5 & 15 & 25 & 35 & 45 & - \\
$d_{3}$ - temperature & 35 & 42 & 49 & 56 & 63 & ${ }^{\circ} \mathrm{C}$ \\
$d_{4}$ - point-to-point delay & 31 & 41 & 51 & 61 & 71 & $\mathrm{~ms}$ \\
$d_{5}$ - payload mass error & -9 & -6 & -3 & 0 & 3 & $\%$ \\
\hline
\end{tabular}

Table 4: Model fits for a robot and test-cycle.

\begin{tabular}{ccccc} 
& & \multicolumn{3}{c}{ Model Fit [\%] } \\
\cline { 3 - 5 } FDA & Model Eq. & Axis 1 & Axis 2 & Axis 3 \\
\hline$A$ & $(24)$ & 83.2 & 72.5 & 82.1 \\
$A$ & $(3)$ & 87.9 & 83.7 & 88.1 \\
$B$ & $(24)$ & 64.6 & 65.5 & 65.7 \\
$B$ & $(3)$ & 87.8 & 91.0 & 91.9 \\
$C$ & $(24)$ & 89.8 & 84.2 & 85.6 \\
$C$ & $(3)$ & 95.0 & 85.3 & 89.9 \\
\hline
\end{tabular}

\section{Design parameters}

According to C-5, the test quantities produce comparable results only when the same FDA, robot, axis and test-cycle are used. Therefore, for different combinations of these design parameters, a different regression model should be used. This gives a total of $3 \times 2 \times 3 \times 6=108$ regression models corresponding to the respective number of FDAs, robots, axes and test-cycles considered. Notice though that the same design matrices can be used to identify all regression models. And further that the same simulated data for a robot can be used to identify the models for all FDAs and for all axes. Each regression model requires $N=45$ experiments, a total of $108 /(3 \times 3) \times 45=540$ simulations are therefore needed to identify all regression models in the study. Each simulation experiment takes around ten seconds to be performed, requiring $1 \mathrm{~h} 30 \mathrm{~min}$ for all 540 simulations.

\section{Identification and validation}

The simulation experiments are performed and the regression models are identified using (5). The design matrix used for identification, given in Table 2, is also used for validation of the regression models but with different factor levels, given in Table 3. The model fits, computed as in (10), are shown in Table 4 for a certain robot and test-cycle. The fits are generally high for all FDAs, with higher values for the full second-order model, specially for FDA $B$.

\subsection{Determining relevant factors}

Sensitivity analysis is used to address Q-1, i.e., to determine which input factors cause more variations to the output of an FDA. Because model (24) is simpler to analyze than model (3), only the coefficients for this model are shown here. 
The regression coefficients are normalized as in (12) and are grouped for each FDA according to (13). Each of the group matrices have dimensions $(36 \times 21)$, corresponding to the combination of the design parameters left and number of coefficients in the regression model respectively.

In Figure 2, the 21 normalized coefficients are displayed in box plots for each test quantity. The statistics for the box plots are computed over each column of the group matrices. Recall that, because of the normalization used, coefficients with magnitudes larger than 1 indicate that the corresponding regression term has a larger effect to the output compared to the direct effect of $f$, i.e., wear. From the figures, it is possible to note that the coefficient $\bar{\eta}_{3}$ considerably affects all FDAs. This coefficient relates to $d_{3}$, i.e., the direct effect of temperature. FDA $C$ presents the lowest value for the median of $\bar{\eta}_{3}$. Coefficient $\bar{\eta}_{5}$, associated to $d_{5}$, i.e., payload mass error, also gives significant responses for FDA A but are generally small for FDAs $B$ and $C$. The coefficients associated with the effects of wear in other joints, $d_{1}$ and $d_{2}$, and point-to-point delay $d_{4}$, show less significant responses for all FDAs.

\section{Possible extensions}

By including tuning variables as input factors to the surrogate models, the sensitivity of an FDA to tuning can be investigated in the same manner.

The outliers present in the box plots for the coefficient groups should be investigated in detail as they may reveal important properties of the problem and FDA. There might be combinations of the design parameters that increases or reduces the sensitivity to certain factors. This type of information is useful for further development and verification of FDAs.

\subsection{Comparing fault detection algorithms}

As discussed in Section 4, the SNRs can be seen as an average performance measure for a test quantity. For the computation of the SNRs, the parameters defining the hypotheses in (14) are set as

$$
f^{0}=-0.5, \quad \Delta=1, \quad p(\boldsymbol{d})=\mathcal{N}\left(0, I \sigma^{2}\right), \quad \sigma=0.25,
$$

i.e., the wear fault, $f$, is changed by a fourth of its allowed range and the disturbance factors $\boldsymbol{d}$ are considered as Gaussian random variables independently distributed with a common standard deviation which is $1 / 16$ of their range. Model structure (3) is considered in the study since it presented larger fits in general (recall Table 4). For each regression model, the SNRs are computed based on $110^{5}$ $\mathrm{MC}$ runs. Using the surrogate models, the total $1.0810^{7} \mathrm{MC}$ runs needed for all regression models took approximately 12 seconds in a standard desktop computer. To perform the same analysis using Level 2 studies, i.e., with simulations, would have taken nearly three and a half years.

The SNRs grouped according to FDA are displayed as box plots in Figure 3. The SNRs can be used to rank the different FDAs. If the median over each group is used as a criterion, this example reveals that FDA $C$ gives the best performance. 


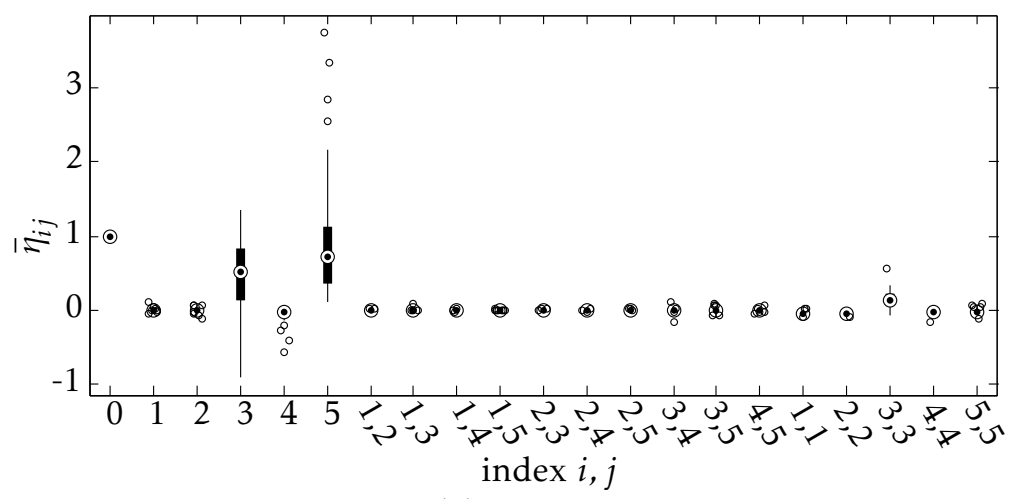

(a) FDA A.

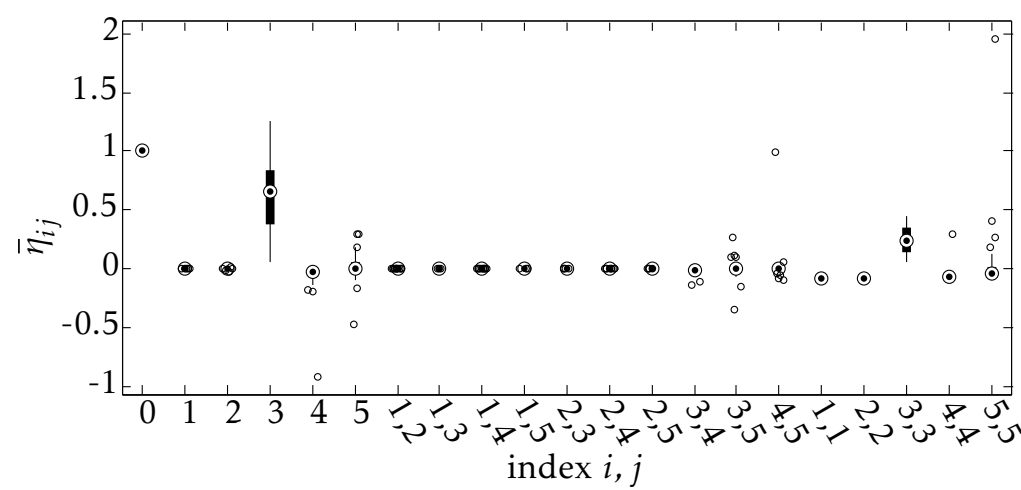

(b) FDA B.

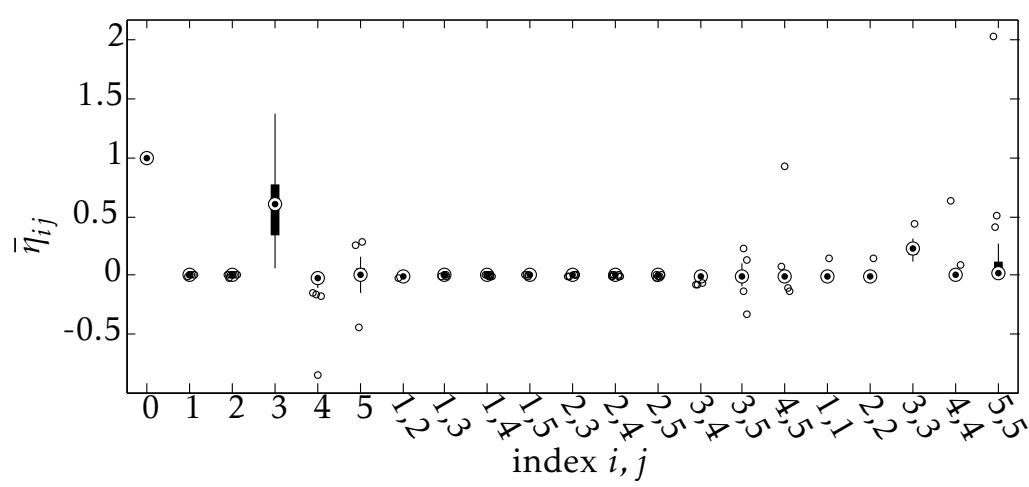

(c) FDA C.

Figure 2: Normalized regression coefficients for model structure (24) grouped according to FDA. In the box plots, the dotted circle indicates the median, the extremities of the bar relate to the 25 th and 75 th percentiles and the isolated circles are outliers. Notice the different scales. 


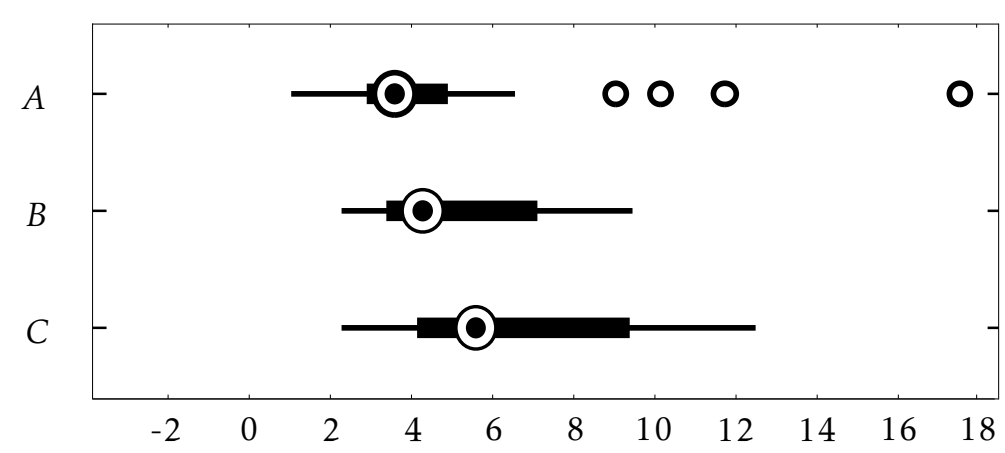

Figure 3: SNRs grouped for the different FDAs. The box plots are for groups over all design parameters where the dotted circle indicates the group median, the extremities of the bar relate to the 25 th and 75 th percentiles and circles are outliers.

\section{Possible extensions}

For some design parameters, FDA $A$ gives very large SNR values. These cases correspond to outliers in the box plots and could be investigated further as they may reveal useful information about the FDA.

This type of analysis can also provide criteria for the choice of design parameters. For instance, the SNRs can be grouped according to test-cycles to reveal whether there are test-cycles that facilitate the distinction of faults in general or for a specific FDA. Tuning variables can also be seem as a design parameter, in which case different regression models are found for different values of the tuning parameters. In this setting, the SNRs for a certain FDA can be grouped according to the tuning parameters, providing a criterion for the selection of the tuning parameters.

\subsection{Determining the effective scope}

The use of scope matrices is illustrated here to determine how the factors $d_{3}$ and $d_{5}$, i.e., temperature and payload mass error, delimit the scope of the test quantities. Due to its natural interpretation, criterion (17) is considered with $P_{f}^{\prime}=0.01$ and $P_{d}^{\prime}=0.99$ for a decision rule given by the threshold check (18). The hypotheses in (20) are defined with $f^{0}=-2, d_{j \neq i}^{\prime}$ are set to the nominal values given in (23) and $d_{i} \sim p(d)=\mathcal{N}\left(0, \sigma^{2}\right)$ is a Gaussian distribution with zero mean and standard deviation $\sigma$. The criterion is evaluated for values of $\Delta \in[0,4]$ and $\sigma \in[0.01,1]$ based on a linear grid of size $30 \times 30$. The hypotheses densities are estimated using a kernel density estimator based on $110^{5} \mathrm{MC}$ runs. The total MC runs needed for the study is of $30 \times 30 \times 1.0810^{7}=9.7210^{9}$ which took approximately $3 \mathrm{~h} 15 \mathrm{~min}$ using the surrogate models. To evaluate the analysis at Level 2, with simulations, would have taken more than three millennia.

Group scope matrices are formed for each FDA. An entry in the resulting matrix 

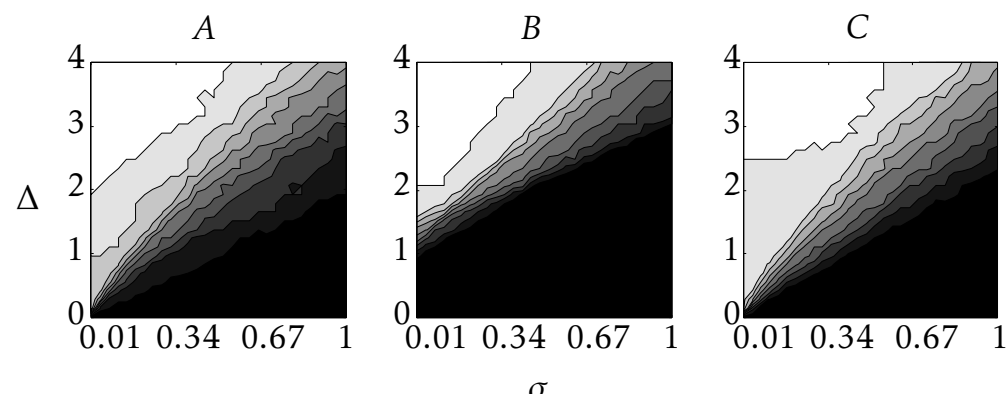

(a) Random temperature disturbances $d_{3}$.
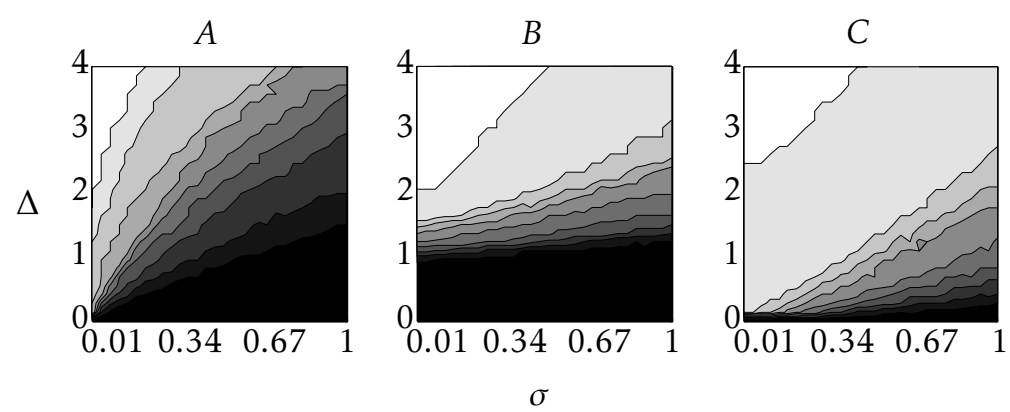

(b) Random payload mass error disturbances $d_{5}$.

Figure 4: Visualization of the scope matrices grouped according to FDA. The colormap relates to how often the performance test was successful, varying from 0 (black) to 36 (white). The clearer the plot, the more often an FDA performed satisfactorily. 
can take values between zero and 36 . The resulting matrices for disturbances of temperature, $d_{3}$, and payload mass error, $d_{5}$, are shown in Figure 4 with a colormap associated to the entry value in the scope matrix. The clearer the graph, the more often the FDA performs satisfactorily for the related combination of change size $\Delta$ and standard deviation of disturbance $\sigma$. From an inspection of the figures, it is possible to determine the minimal size of $\Delta$ for which an FDA performs satisfactorily given a fixed disturbance variation $\sigma$, and vice-versa. From Figure $4 \mathrm{~b}$, it is possible to note that FDA $\mathrm{C}$ is the least affected by payload disturbances. As seen in Figure 4a, all test quantities are considerably affected by temperature, but FDAs $A$ and $C$ allow for more variations of temperature compared to FDA $B$.

\section{Possible extensions}

The hypotheses defined in (20) allow for variation of only two factors at a time, the fault $f$ and a disturbance factor of choice $d_{i}$. In principle, any number of the $n$ factors can be changed at the same time. The same type of analysis can be considered, although alternative visualization techniques may be needed.

\section{Conclusions}

This paper proposed a framework for evaluation and comparison of fault detection algorithms (FDAs) based on simulations. An extensive investigation of the different FDAs is made possible with the use of surrogate models which considerably reduces the time needed for the evaluation study. As illustrated in the application example, this was in fact the only viable alternative. The approaches suggested may be used to reveal which inputs affect an FDA the most, which FDA performs best in average and the effective scope of an FDA. The framework is rather general and can be extended to study various aspects of fault detection algorithms.

It should be stressed that conclusions drawn based on simulations or surrogate models should always be carried out carefully since they are a limited representation of reality. Results achieved in this manner give good insights about the problem and support decisions but, ultimately, the fault detection algorithms should be evaluated based on real experiments. In the robotics application, accelerated wear tests can be used with this purpose, but with much higher costs and time required for a statistically significant study. 


\section{Bibliography}

A. Atkinson, A. Donev, and R. Tobias. Optimum Experimental Designs, with SAS. Oxford University Press, Cary, USA, 2007.

M. Basseville and I. V. Nikiforov. Detection of abrupt changes: theory and application. Prentice-Hall, Inc., Upper Saddle River, NJ, USA, 1993.

A. C. Bittencourt and P. Axelsson. Modeling and experiment design for identification of wear in a robot joint under load and temperature uncertainties based on friction data. IEEE/ASME Transactions on Mechatronics, 19(5):1694-1706, October 2014.

G. E. P. Box and K. B. Wilson. On the experimental attainment of optimum conditions. Journal of the Royal Statistical Society. Series B (Methodological), 13 (1):1-45, 1951 .

G. E. P. Box, W. G. Hunter, and J. S. Hunter. Statistics for Experimenters. John Wiley and Sons, 1978.

V. V. Fedorov. Theory of optimal experiments. Academic Press, 1972.

P. Frank and X. Ding. Survey of robust residual generation and evaluation methods in observer-based fault detection systems. Journal of Process Control, 7(6): 403-424, 1997.

F. Gustafsson. Adaptive Filtering and Change Detection. Wiley, October 2000.

R. Isermann. On the applicability of model-based fault detection for technical processes. Control Engineering Practice, 2(3):439-450, 1994.

R. Isermann. Fault-Diagnosis Systems - An Introduction from Fault Detection to Fault Tolerance. Springer, 1st edition, 2006.

J. P. C. Kleijnen, S. M. Sanchez, T. W. Lucas, and T. M. Cioppa. A User's Guide to the Brave New World of Designing Simulation Experiments. INFORMS Journal on Computing, 17, 2005.

X. Li and K. Zhou. A time domain approach to robust fault detection of linear time-varying systems. Automatica, 45(1):94-102, 2009.

N. Liu and K. Zhou. Optimal robust fault detection for linear discrete time systems. Journal of Control Science and Engineering, 7:1-16, January 2008.

L. Ljung. System Identification: Theory for the User. Prentice Hall PTR, 2nd edition, December 1999.

J. E. Oakley and A. O’Hagan. Probabilistic sensitivity analysis of complex models: a Bayesian approach. Journal of the Royal Statistical Society: Series B (Statistical Methodology), 66(3):751-769, 2004.

A. Saltelli, M. Ratto, T. Andres, F. Campolongo, J. Carboni, M. S. D. Gatelli, and 
S. Tarantola. Global Sensitivity Analysis. The Primer. John Wiley and Sons, 2008.

S. Sanchez. Work smarter, not harder: guidelines for designing simulation experiments. In Proceedings of the 2006 Winter Simulation Conference, Monterey, USA, December 2006.

X. Wei and M. Verhaegen. Robust fault detection observer design for linear uncertain systems. International Journal of Control, 84(1):197-215, 2011. 


\section{Paper E}

\section{Data-Driven Anomaly Detection based on a Bias Change}

Authors:

André Carvalho Bittencourt and Thomas Bo Schön.

Edited version of the paper:

A. C. Bittencourt and T. Schön. Data-driven anomaly detection based on a bias change. In Proceedings of the 19th IFAC World Congress, Cape Town, South Africa, 2014. 



\title{
Data-Driven Anomaly Detection based on a Bias Change
}

\author{
André Carvalho Bittencourt* and Thomas Bo Schön** \\ ${ }^{*}$ Dept. of Electrical Engineering, \\ Linköping University, \\ SE-581 83 Linköping, Sweden
${ }^{* *}$ Dept. of Information Technology
Uppsala University \\ SE-751 05 Uppsala, Sweden
}

\begin{abstract}
This paper proposes batch and sequential data-driven approaches to anomaly detection based on generalized likelihood ratio tests for a bias change. The procedure is divided into two steps. Assuming availability of a nominal dataset, a nonparametric density estimate is obtained in the first step, prior to the test. Second, the unknown bias change is estimated from test data. Based on the expectation maximization (EM) algorithm, batch and sequential maximum likelihood estimators of the bias change are derived for the case where the density estimate is given by a Gaussian mixture. Approximate asymptotic expressions for the probabilities of error are suggested based on available results. Simulations and real world experiments illustrate the approaches.
\end{abstract}

\section{Introduction}

In anomaly detection, the main objective is to determine whether observations collected from a system conform to expected (normal) behavior or not (i.e., an anomaly). Anomaly detection appears in a variety of applications, such as condition monitoring of machines, fraud detection, intrusion detection, etc. A survey in the topic is provided by Chandola et al. (2009). A factor that distinguishes anomaly detection to related detection problems is the lack of knowledge of the anomaly. This is a rather common situation, e.g., in condition monitoring and fault detection. In many cases, anomaly detection is done with the use of a statistical model to describe the nominal behavior of the observations. An anomaly can be inferred in case the observed data do not conform to the model. In many practical situations, however, it can be difficult to determine the statistical model for the observations. A common situation is that it is possible to collect measurements (data) under normal conditions. This nominal dataset contains relevant information about the conforming behavior of the system and it is possible to infer the presence of an anomaly based only on nominal data. 
Examples of data-driven approaches to anomaly detection are one-class classification algorithms, e.g., Devroye and Wise (1980); Schölkopf et al. (2001), where a boundary region in the observation space is determined from a nominal dataset. Fresh observations falling outside this region are classified as anomalies. A shortcoming with such an approach is that all knowledge about the normal behavior is summarized by a region in the observation space. For instance, this approach would fail to recognize that if observations consistently fall in a low probability region of the support, it is more likely that an anomaly is present. An alternative is to estimate a model of the measurements density based on the nominal data. In this case, anomalies can be detected based on the probability that test data have under the estimated density model. Since it is often difficult to determine the family of distributions, flexible density models are commonly used, such as mixture models (Agarwal, 2007), as well as nonparametric estimates (Desforges et al., 1998; Yeung and Chow, 2002). A shortcoming with approaches based on a model solely for the nominal behavior is that it is not possible to provide an estimate of how certain the test is of the presence of an anomaly. This type of information is however often important in practice to support decisions of recovery actions.

With the possibility to determine probabilistic models for both the normal and abnormal behaviors, it is possible to quantify the decision uncertainties since probabilistic models are defined for the entire problem. Anomaly detection can be seen as a hypothesis testing problem (HTP). In a binary HTP, the null hypothesis $\mathcal{H}^{0}$ describes the nominal behavior and the alternative hypothesis $\mathcal{H}^{1}$ describes the abnormal behavior. The hypotheses are described by the statistical behavior of the measurements $y \in \mathbb{R}^{d}$ under each hypothesis,

$$
\mathcal{H}^{0}: y \sim p^{0}(y), \quad \mathcal{H}^{1}: y \sim p^{1}(y) .
$$

When the hypotheses densities, $p^{0}(y)$ and $p^{1}(y)$, are given or when their family of parametric distributions are known, there are well-established statistical tests based on likelihood ratios, i.e.,

$$
\Lambda(y) \triangleq p^{1}(y) / p^{0}(y)
$$

see, e.g., Neyman and Pearson (1933); Wald (1945).

An approach to overcome the lack of knowledge for the anomaly is to define it as a change relative to nominal. In this manner, the available knowledge about the nominal behavior can be used to test for an anomaly. Here, a bias (location) change is considered, i.e., the density for the alternative hypothesis is written as

$$
p^{1}(y)=p^{0}(y-\Delta)
$$

for an unknown bias change $\Delta$. Using this model, this article aims at providing an approach for anomaly detection that without requiring specification of a density function and based only on availability of a nominal dataset,

- is flexible and can be used for different problems,

- can provide estimates of the decision uncertainties,

- requires only minimal and meaningful specification parameters. 
This is achieved via a two step approach. First, the nominal dataset is used to find a nonparametric estimate of the density function for $\mathcal{H}^{0}$, denoted $\widehat{p}^{0}(y)$. In the second step, incoming test measurements are used to find a maximum likelihood estimate $\widehat{\Delta}$ of the unknown bias change. These estimates are used to define the approximate models

$$
\begin{aligned}
& \mathcal{H}^{0}: y \sim \widehat{p}^{0}(y), \\
& \mathcal{H}^{1}: y \sim \widehat{p}^{1}(y \mid \widehat{\Delta})=\widehat{p}^{0}(y-\widehat{\Delta}),
\end{aligned}
$$

which are tested based on a generalized likelihood ratio (GLR) test assuming this model to be true. Both batch and sequential tests are devised.

The presentation is organized as follows, Section 2 presents the bias change model and reviews the GLR test. Section 3 presents the approaches used to find the estimate $\widehat{p}^{0}(y)$ based on a nominal dataset. The resulting density model will be a finite mixture distribution. Section 4 defines maximum likelihood estimators for $\Delta$ based on the Expectation Maximization algorithm. Algorithms are derived for mixtures of the multivariate Gaussian distribution. The use of GLR tests based on the approximate models (3) is illustrated in Section 5 through simulations and real data examples followed by concluding remarks.

\section{The Bias Change Model and the GLR test}

The assumption that an anomaly will appear as a bias change from nominal gives the following hypotheses

$$
\begin{aligned}
& \mathcal{H}^{0}: y \sim p^{0}(y), \\
& \mathcal{H}^{1}: y \sim p^{1}(y \mid \Delta)=p^{0}(y-\Delta),
\end{aligned}
$$

for the unknown bias vector $\Delta$. The expected value of $y$ under $\mathcal{H}^{1}$ is

$$
\mathbb{E}_{p^{1}}[y]=\mathbb{E}_{p^{0}}[y]+\Delta
$$

i.e., the mean is changed by $\Delta$. This model is easy to interpret and bias changes are often considered when detecting anomalies, e.g., in the literature of fault diagnosis (Isermann, 2006). The model describes situations where the data are shifted in the observation space. The parameter $\Delta$ also carries valuable information about the problem. For instance, if $\Sigma$ is the density covariance, then $\Delta^{T} \Sigma^{-1} \Delta$ measures the significance of the change relative to the density volume, similar to a signal to noise ratio.

Introducing the notation $Y_{i: j}=\left[y_{i}, \cdots, y_{j}\right](j>i)$, for $j-i+1$ independent and identically distributed (i.i.d.) measurement vectors $y_{i} \in \mathbb{R}^{d}$ and let $Y_{N} \triangleq Y_{1: N}$, the objective is to decide whether a given sample $Y_{N}$ belongs to $\mathcal{H}^{0}$ or $\mathcal{H}^{1}$ in (4). 
This can be done with a generalized (log-) likelihood ratio (GLR),

$$
\begin{aligned}
\widehat{s_{N}} & \triangleq \log \widehat{\Lambda}_{N}\left(Y_{N}\right)=\max _{\Delta} \log \frac{p_{N}^{1}\left(Y_{N} \mid \Delta\right)}{p_{N}^{0}\left(Y_{N}\right)} \\
& =\log \frac{p_{N}^{1}\left(Y_{N} \mid \widehat{\Delta}_{N}\right)}{p_{N}^{0}\left(Y_{N}\right)}=\sum_{j=1}^{N} \log \frac{p^{0}\left(y_{j}-\widehat{\Delta}_{N}\right)}{p^{0}\left(y_{j}\right)},
\end{aligned}
$$

where $\widehat{\Delta}_{N}$ is a maximum likelihood (ML) estimate of the unknown bias. Batch estimation of $\Delta$ is discussed in Section 4.1. The GLR is tested based on a threshold check,

$$
\widehat{s_{N}} \underset{\mathcal{H}^{0}}{\stackrel{\mathcal{H}^{1}}{\gtrless} \hbar,}
$$

the above notation means that $\mathcal{H}^{0}$ is chosen if the test statistic ${\widehat{s_{N}}}_{\mathrm{N}}$ is smaller or equal to the threshold $\hbar$ and otherwise $\mathcal{H}^{1}$ is chosen.

\subsection{Unknown change time}

The GLR quantity used in (6) assumes that the entire batch $Y_{N}$ was collected under either $\mathcal{H}^{0}$ or $\mathcal{H}^{1}$, i.e., the change time is known. In many practical situations, the change time is unknown. For a batch $Y_{N}$, there are $N+1$ possibilities, either no change was present or a change appeared at any $t \in\{1, \ldots, N\}$. A hypothesis test for this problem can be defined according to (Basseville and Nikiforov, 1993, Section 2.6.1),

$$
\begin{aligned}
& \mathcal{H}^{0}: y_{i} \sim p^{0}(y), 1 \leq i \leq N, \\
& \mathcal{H}^{1}: \begin{cases}y_{i} \sim p^{0}(y), & 1 \leq i \leq t-1, \\
y_{i} \sim p^{1}(y \mid \Delta)=p^{0}(y-\Delta), & t \leq i \leq N,\end{cases}
\end{aligned}
$$

where both $t$ and $\Delta$ are unknown in $\mathcal{H}^{1}$. The resulting GLR is based on a joint maximization of the unknowns as,

$$
\begin{aligned}
\widetilde{s_{N}} & \triangleq \max _{1 \leq t \leq N} \max _{\Delta} \log \frac{p_{1: t-1}^{0}\left(Y_{1: t-1}\right) p_{t: N}^{1}\left(Y_{t: N} \mid \boldsymbol{\Delta}\right)}{p_{N}^{0}\left(Y_{N}\right)} \\
& =\max _{1 \leq t \leq N} \log \frac{p_{t: N}^{1}\left(Y_{t: N} \mid \widehat{\Delta}_{t: N}\right)}{p_{t: N}^{0}\left(Y_{t: N}\right)}=\max _{1 \leq t \leq N} \widehat{s_{t: N}},
\end{aligned}
$$

and the hypotheses are chosen as in (7) with $\widehat{s_{N}}$ replaced by $\widetilde{s_{N}}$. An estimate of the change time is,

$$
\widehat{t}_{N}=\arg \max _{1 \leq t \leq N} \widehat{s_{t: N}},
$$

and the estimate for the change is $\widehat{\Delta}_{\widehat{t}_{N}: N}$. The statistic ${\widetilde{s_{N}}}_{\text {requires finding the } \mathrm{ML}}$ estimate of $\widehat{\Delta}_{t: N}$ for $N$ possible splits $t \in\{1, \ldots, N\}$ and evaluation of the related $\log$-likelihood ratios $\widehat{s_{t: N}}$. 


\subsection{Sequential solution}

The formulation in (8) and (9) is a batch approach, i.e., the entire sequence $Y_{N}$ is needed. A sequential approach is however possible by repeating the procedure for each incoming measurement sequence $\left\{y_{n}\right\}$ (Gustafsson, 2000, Section 3.5.3). Every time new data $y_{n}$ are received, $n$ estimates $\widehat{\Delta}_{t: n}$ for $t \in\{1, \ldots, n\}$, are found and the associated $\widehat{s}_{t: n}$ are evaluated. The complexity therefore increases with $n$.

The denominator for ${\widehat{s_{t}}}_{n}$ in (6), i.e., the log-likelihood function for $\mathcal{H}^{0}$, can be computed recursively under the i.i.d. assumption. Given the previous value $\log p_{t: n-1}^{0}\left(Y_{t: n-1}\right)$ and $y_{n}$, it can be updated as,

$$
\log p_{t: n}^{0}\left(Y_{t: n}\right)=\log p_{t: n-1}^{0}\left(Y_{t: n-1}\right)+\log p^{0}\left(y_{n}\right) .
$$

The numerator for ${\widehat{s_{t}}}_{n}$, i.e., the log-likelihood function for $\mathcal{H}^{1}$, must however be evaluated for the entire $Y_{t: n}$ based on an updated $\widehat{\Delta}_{t: n}$ which, in general, cannot be found sequentially. The estimate $\widehat{\Delta}_{t: n}$ requires solution of an optimization problem and is more computationally demanding than the evaluation of the log-likelihood function for $\mathcal{H}^{1}$. To reduce complexity, the estimates $\widehat{\Delta}_{t: n}$ can be found recursively in the data, i.e., $\widehat{\Delta}_{t: n}$ is found based only on the previous value $\widehat{\Delta}_{t: n-1}$ and current measurement $y_{n}$. Recursive estimation of $\Delta$ is discussed in Section 4.2.

\subsection{Asymptotic performance}

Associated to any test is the probability of deciding incorrectly for $\mathcal{H}^{0}$, denoted $P_{m}$, and the probability of deciding incorrectly for $\mathcal{H}^{1}$, denoted $P_{f}$. For a GLR test they are given by $P_{m}=\operatorname{Pr}\left(\widehat{s}_{N} \leq \hbar \mid \mathcal{H}^{1}\right)$ and $P_{f}=\operatorname{Pr}\left(\widehat{s}_{N}>\hbar \mid \mathcal{H}^{0}\right)$. While no analytical solution is available in general, the error probabilities can in principle be found based on Monte Carlo integration. An alternative is to find $P_{f}$ and $P_{m}$ based on the asymptotic behavior of the GLR statistic. The asymptotic behavior of the test statistic is given by (Mackay, 2003, App. 6A-C),

$$
\begin{aligned}
& 2 \widehat{s}_{N} \mid \mathcal{H}^{0} \stackrel{\text { as. }}{\sim} \mathcal{X}_{d}^{2}, \\
& 2 \widehat{s}_{N} \mid \mathcal{H}^{1} \stackrel{\text { as. }}{\sim} \mathcal{X}_{d}^{\prime 2}(\lambda(\Delta)), \quad \lambda(\Delta) \triangleq \Delta^{T} \mathcal{F}(\mathbf{0}) \Delta,
\end{aligned}
$$

where $\Delta$ is the true bias change, $\mathcal{X}_{d}^{2}$ is the chi-square distribution with $d$ degrees of freedom, $\mathcal{X}_{d}^{\prime 2}(\lambda)$ is the non-central chi-square with non-centrality parameter $\lambda$ and $\mathcal{F}(\mathbf{0})$ is the Fisher information matrix for $\Delta$ evaluated at zero. This result is valid whenever the correct models are used and $\widehat{\Delta}_{N}$ tends to the true value $\Delta$. Since the asymptotic behavior of the test statistic does not depend on unknowns under $\mathcal{H}^{0}$, a threshold can be found from (12a) for a desired error level $P_{f}^{\prime}$. An estimate of $P_{m}$ can also be computed by using the maximum likelihood estimate $\widehat{\Delta}$ 
in (12b). This is summarized as follows

$$
\begin{aligned}
\hbar\left(P_{f}^{\prime}\right) & =\inf \left\{\hbar \in \mathbb{R}: \int_{\hbar}^{\infty} \mathcal{X}_{d}^{2}(s) \mathrm{d} s \leq P_{f}^{\prime}\right\}, \\
P_{m}\left(P_{f}^{\prime}\right) & =\int_{-\infty}^{\hbar\left(P_{f}^{\prime}\right)} \mathcal{X}_{d}^{\prime 2}(s ; \lambda(\widehat{\Delta})) \mathrm{d} s .
\end{aligned}
$$

To apply the GLR test for the bias change model, the unknown density $p^{0}(y)$ is needed. In a practical setup, it is often common to introduce assumptions on the data distribution, the Gaussian model being a common choice. Although the Gaussian model gives statistical tests that can be conveniently described by sufficient statistics (Van Trees, 2001), it is clear that there will be situations where this model is a poor description of $\mathcal{H}^{0}$. In this paper, no assumption is forced about $\mathcal{H}^{0}$, instead, all knowledge is considered to be contained in a nominal dataset $Y_{N_{0}}^{0}$. The next section describes approaches where this dataset is used to find an approximate model $\widehat{p}^{0}(y)$ for $\mathcal{H}^{0}$. Using this approximation, Section 4 describes methods to find maximum likelihood estimates of the bias change $\widehat{\Delta}$. The density and bias estimates define the approximate models in (3) which are tested based on a GLR test assuming it is the true model. The only specification parameter needed is the desired error level $P_{f}^{\prime}$ to find the threshold $\hbar$ as in (13a). The associated error probabilities are found based on the asymptotic expressions in (12) for the approximate models.

\section{Nonparametric Density Estimators}

A nominal dataset $Y_{N_{0}}^{0}$ with $N_{0}$ i.i.d. observations from $\mathcal{H}^{0}$ is considered available and is used to find a nonparametric density estimate $\widehat{p}^{0}(y)$ for $\mathcal{H}^{0}$. The density model will take the form of a finite mixture

$$
\widehat{p}^{0}(y)=\sum_{k \in \mathbb{K}} \pi_{k} \kappa\left(y ; y_{k}^{0}, \boldsymbol{h}\right), \quad \sum_{k \in \mathbb{K}} \pi_{k}=1, \pi_{k}>0,
$$

where $\mathbb{K}$ is an index set with cardinality $|\mathbb{K}|=K \leq N_{0}, \kappa(\cdot)$ is a positive kernel function that integrates to one. The bandwidth $\boldsymbol{h} \in \mathbb{R}^{d}$ is fixed and the weighting coefficients $\left\{\pi_{k}\right\}$ are found according to the chosen density estimator. Two nonparametric density estimators are discussed next.

\subsection{Kernel density estimator}

The first type of estimator considered is a so called kernel density estimator (KDE), or Parzen estimator. The KDE based on the nominal dataset $Y_{N_{0}}^{0}$ is given by a finite 
mixture model (14) with

$$
\begin{array}{r}
\mathbb{K}=\left\{1,2, \ldots, N_{0}\right\}, \quad \pi_{k}=\frac{1}{N_{0}}|S(\boldsymbol{h})|^{-1 / 2} \\
\kappa\left(y ; y_{k}^{0}, \boldsymbol{h}\right)=\kappa\left(S(\boldsymbol{h})^{-1 / 2}\left(y-y_{k}^{0}\right)\right),
\end{array}
$$

where $S(\boldsymbol{h})$ is a positive definite scaling matrix. The KDE model has as many components as data points and the coefficients $\left\{\pi_{k}\right\}$ are fixed and identical. As shown by Parzen (1962); Cacoullos (1966), this estimator is consistent and asymptotically unbiased. The KDE method requires specification of the bandwidth $\boldsymbol{h}$. There are several approaches reported in the literature for bandwidth selection (Jones et al., 1996b,a). Here, a diagonal $S(\boldsymbol{h})$ will be considered with bandwidth elements chosen using Silverman's rule of thumb (Silverman, 1986),

$$
\begin{aligned}
S(\boldsymbol{h}) & =\operatorname{diag}(\boldsymbol{h}) \\
\sqrt{h_{i}} & =\frac{4}{d+2}^{\frac{1}{d+4}} N_{0}^{\frac{-1}{d+4}} \widehat{\sigma}_{i},
\end{aligned}
$$

for $i=\{1, \ldots, d\}$ and where $\widehat{\sigma}_{i}$ is an estimate of the data standard deviation over the $i$ th dimension.

Besides requiring storage of the entire nominal dataset, performing inference with a KDE will become computationally intensive when $N_{0}$ is large. An alternative is to consider reduced mixture models, with $K \ll N_{0}$ components. When the number of components $K$ is fixed, it is possible to find maximum likelihood estimates for the parameters using, e.g., the EM algorithm by Dempster et al. (1977). A disadvantage with such an approach is that the number of components $K$ must be pre-specified.

\subsection{A sparse density estimator}

An alternative will be considered here that requires only few a components to describe the density estimate. It is based on the generalized cross entropy (GCE) method presented by Botev and Kroese (2011), which does not require specification of $K$ or $\boldsymbol{h}$. For a dataset $Y_{N_{0}}^{0}$, the estimate is given as

$$
\widehat{p}^{0}(y)=\sum_{k \in \mathbb{K}} \lambda_{k}^{*} \kappa\left(y ; y_{k}^{0}, \boldsymbol{h}^{*}\right),
$$

with $\mathbb{K}=\left\{1, \ldots, N_{0}\right\}, \lambda^{*}$ is a sparse weight vector which, together with the bandwidth $\boldsymbol{h}^{*}$, is given by

$$
\left(\boldsymbol{h}^{*}, \lambda^{*}\right)=\left\{(\boldsymbol{h}, \lambda): \mathbf{1}^{T} \lambda(\boldsymbol{h})=1, \lambda(\boldsymbol{h})=\arg \min _{\lambda \geq 0} \lambda^{T} C(\boldsymbol{h}) \lambda-\lambda^{T} \widehat{\boldsymbol{\phi}}(\boldsymbol{h})\right\}
$$


The quadratic program (QP) for $\lambda(\boldsymbol{h})$ is defined by

$$
\begin{aligned}
\widehat{\phi}_{i}(\boldsymbol{h}) & =\frac{1}{N_{0}-1} \sum_{j \neq i} \kappa\left(y_{j}^{0} ; y_{i}^{0}, \boldsymbol{h}\right), \quad i=1, \ldots, N_{0}, \\
{[C(\boldsymbol{h})]_{i j} } & =\int_{\mathbb{R}^{d}} \kappa\left(y ; y_{i}^{0}, \boldsymbol{h}\right) \kappa\left(y ; y_{j}^{0}, \boldsymbol{h}\right) \mathrm{d} y,
\end{aligned}
$$

and $C(\boldsymbol{h}) \in \mathbb{R}^{N_{0} \times N_{0}}$ is positive definite by construction.

This approach is algorithmically similar to the support vector density estimator by Vapnik and Mukherjee (2000), in which the condition $\mathbf{1}^{T} \boldsymbol{\lambda}(\boldsymbol{h})=1$ is included as a constraint in the QP and $\boldsymbol{h}$ is pre-specified. As noted by Botev and Kroese (2011), the QP in (18a) is closely related to the support vector regression problem with an $\epsilon$-insensitive error function, see, e.g., (Bishop, 2006, Section 7.1.4), and most elements in $\lambda^{*}$ will be close to zero.

\section{Computing the estimate}

To avoid solving (18a) for a $d$-dimensional $\boldsymbol{h}$, a simplification is made which considers a scalar bandwidth $h$ applied to a vector containing an estimate of the variance along each dimension, i.e.,

$$
\boldsymbol{h}^{T}=h\left[\widehat{\sigma}_{1}^{2}, \cdots, \widehat{\sigma}_{d}^{2}\right],
$$

where $\widehat{\sigma}_{i}$ is an estimate of the standard deviation along the $i$ th dimension. In this manner, only one bandwidth parameter needs to be found and different scalings are allowed for the different dimensions. The resulting problem (18a) is solved by addressing the nonlinear least squares

$$
h^{*}=\arg \min _{h}\left(\mathbf{1}^{T} \lambda(h)-1\right)^{2},
$$

where $\lambda(h)$ is the solution to the QP $(18 \mathrm{a})$ and $\lambda^{*}=\lambda\left(h^{*}\right)$.

To remove small components in $\lambda^{*}$, a pruning approach is suggested here. Let $\lambda^{*}$ be ordered as $\lambda_{1}^{*} \leq \lambda_{2}^{*} \leq \ldots \lambda_{N_{0}}^{*}$, the $\epsilon$ approximation of (17) is written by replacing $\mathbb{K}$ and $\lambda_{k}^{*}$ in (17) with $\mathbb{K}_{\epsilon}$ and $\pi_{k}^{*}$ respectively, where

$$
\mathbb{K}_{\epsilon}:\left\{1 \leq k \leq N_{0}: \sum_{j=1}^{k} \lambda_{j}^{*} \geq \epsilon\right\}, \quad \pi_{k}^{*} \triangleq \frac{\lambda_{k}^{*}}{\sum_{j \in \mathbb{K}_{\epsilon}} \lambda_{j}^{*}}
$$

and $\left|\mathbb{K}_{\epsilon}\right|=K$ will typically be much smaller than the number of data samples $N_{0}$.

\section{Multivariate Gaussian kernel}

The GCE method requires solution of $[C(\boldsymbol{h})]_{i j}$ in $(18 \mathrm{c})$, which is not always analytically tractable. For the Gaussian case, i.e., $\kappa\left(y ; y_{k}^{0}, \boldsymbol{h}\right)=\mathcal{N}\left(\boldsymbol{y} ; y_{k}^{0}, S(\boldsymbol{h})\right)$, it can be 
shown from completion of the squares that

$$
[C(\boldsymbol{h})]_{i j}=\mathcal{N}\left(y_{i}^{0} ; y_{j}^{0}, 2 S(\boldsymbol{h})\right),
$$

see the Appendix for a proof. With $C(\boldsymbol{h})$ found as in (22) with a diagonal $S(\boldsymbol{h})$ as in (16a) and $\boldsymbol{h}$ given as (19), the optimal weights $\lambda^{*}=\lambda\left(h^{*}\right)$ are found from (20) and the approximation (21) is used to present the resulting model as in (14). Notice that the resulting density estimate is a Gaussian mixture model (GMM).

\section{Estimating the Bias Change}

For $\widehat{p}^{0}(y)$ achieved using either the KDE or the GCE methods, the model for the alternative hypothesis in (3) can be written as the finite mixture

$$
\widehat{p}^{1}(y \mid \Delta)=\widehat{p}^{0}(y-\Delta)=\sum_{k=1}^{K} \pi_{k} \kappa_{k}(y-\Delta),
$$

where $\kappa_{k}(y) \triangleq \kappa\left(y ; y_{k}^{0}, \boldsymbol{h}\right)$. The objective of this section is to derive batch and sequential maximum likelihood estimators of $\Delta$ in (23). First, notice that for a mixture density $\widehat{p}(y)$ as given in (14),

$$
\mathbb{E}_{\widehat{p}}[y]=\sum_{k=1}^{K} \pi_{k} \mathbb{E}_{\kappa_{k}}[y]
$$

Using this relation with (5), an estimate of $\Delta$ can be computed based on the test batch $Y_{N}$ from the sample estimate as,

$$
\widehat{\Delta}_{N}^{\mathrm{S}}=\frac{1}{N} \sum_{n=1}^{N} y_{n}-\mathbb{E}_{\widehat{p}^{0}}[y]=\frac{1}{N} \sum_{n=1}^{N} y_{n}-\sum_{k=1}^{K} \pi_{k} \mathbb{E}_{\mathcal{K}_{k}}[y] .
$$

Given that $\mathbb{E}_{\widehat{p}^{0}}[y]=\mathbb{E}_{p^{0}}[y]$, i.e., that the mean for the approximate model corresponds to the true mean in the nominal case, the estimate (24) will converge to the true value. However, for a given sample $Y_{N}$, it does not necessarily maximizes the likelihood function (e.g., if the density is skewed) and an alternative is needed. It is well known that direct optimization of the likelihood function in mixture models is problematic (Bishop, 2006, Section 9.2.1). For mixtures, the EM algorithm can be used to compute maximum likelihood estimates.

\subsection{Batch estimation using EM}

The EM algorithm by Dempster et al. (1977), is a two step iterative procedure for finding maximum likelihood parameter estimates in probabilistic models involving latent (not measured) variables. Let $Z$ and $Y$ denote latent and measured variables respectively, with joint distribution $p(Y, Z \mid \theta)$ governed by the param- 
eter vector $\theta$ and let

$$
\begin{aligned}
Q\left(\boldsymbol{\theta}, \boldsymbol{\theta}^{\prime}\right) & \triangleq \int \log p(Y, Z \mid \boldsymbol{\theta}) p\left(Z \mid Y, \boldsymbol{\theta}^{\prime}\right) \mathrm{d} Z \\
& =\mathbb{E}_{\boldsymbol{\theta}^{\prime}}[\log p(Y, Z \mid \boldsymbol{\theta}) \mid Y] .
\end{aligned}
$$

For iterates $\boldsymbol{\theta}^{(i)}$, the expectation (25) is computed for $Q\left(\boldsymbol{\theta}, \boldsymbol{\theta}^{(i-1)}\right)$ in the E-step. In the $M$-step, the resulting $Q$-function is maximized with respect to $\theta$ to update the iterate $\theta^{(i)}$. The steps are repeated until a convergence criterion is satisfied. The EM algorithm guarantees that the iterates satisfy $p\left(Y \mid \theta^{(i)}\right) \geq p\left(Y \mid \theta^{(i-1)}\right)$ and therefore they converge to a stationary point of the likelihood function.

\section{E-step for mixture models}

As previously noted, the model (23) can be interpreted as a finite mixture model where the parameter $\theta=\Delta$ is common to all mixture components, i.e., it can be written as

$$
\widehat{p}^{1}(y \mid \theta)=\sum_{k=1}^{K} \pi_{k} \kappa_{k}(y \mid \theta), \quad \sum_{k=1}^{K} \pi_{k}=1, \pi_{k}>0 .
$$

Following a typical derivation of the EM algorithm for mixture models, see, e.g., (Bishop, 2006, Section 9.3.1) or Bilmes (1997), a discrete latent variable $z$ with components $z_{k} \in\{0,1\}$ is introduced to assign the unique component of the mixture model that generated the data. The latent variable $z$ is assigned according to the categorical distribution,

$$
p(z)=\prod_{k=1}^{K} \pi_{k}^{z_{k}}
$$

where $p\left(z_{k}=1\right)=\pi_{k}$ and $\sum_{k=1}^{K} \pi_{k}=1$ since only one component can be assigned for each datum. Given the variable $z$, the conditional is

$$
p(y \mid z, \theta)=\prod_{k=1}^{K} \kappa_{k}(y \mid \theta)^{z_{k}},
$$

corresponding to the mixture component that generated the datum. The joint (complete-data) distribution is

$$
p(y, z \mid \theta)=p(y \mid z, \theta) p(z)=\prod_{k=1}^{K}\left[\pi_{k} \kappa_{k}(y \mid \theta)\right]^{z_{k}},
$$

and the marginal over $z$ is

$$
p(y \mid \theta)=\sum_{z} p(y \mid z, \theta) p(z)=\sum_{k=1}^{K} \pi_{k} \kappa_{k}(y \mid \theta),
$$


which is consistent to the original mixture model (26) and

$$
p(\boldsymbol{z} \mid \boldsymbol{y}, \boldsymbol{\theta})=\frac{p(y, \boldsymbol{z} \mid \boldsymbol{\theta})}{p(\boldsymbol{y} \mid \boldsymbol{\theta})}=\frac{\prod_{k=1}^{K}\left[\pi_{k} \kappa_{k}(\boldsymbol{y} \mid \boldsymbol{\theta})\right]^{z_{k}}}{\sum_{j=1}^{K} \pi_{j} \kappa_{j}(y \mid \theta)} .
$$

For a batch with $N$ independent observations $Y_{N}$, the E-step in the EM algorithm is given by the $Q(\cdot, \cdot)$ function,

$$
\begin{aligned}
Q\left(\boldsymbol{\theta}, \boldsymbol{\theta}^{\prime}\right) & =\mathbb{E}_{\boldsymbol{\theta}^{\prime}}\left[\sum_{n=1}^{N} \sum_{k=1}^{K} z_{n k} \log \pi_{k} \kappa_{k}\left(\boldsymbol{y}_{n} \mid \boldsymbol{\theta}\right) \mid Y_{N}\right] \\
& =\sum_{n=1}^{N} \sum_{k=1}^{K} \mathbb{E}_{\boldsymbol{\theta}^{\prime}}\left[z_{n k} \mid Y_{N}\right] \log \pi_{k} \kappa_{k}\left(\boldsymbol{y}_{n} \mid \boldsymbol{\theta}\right) \\
& =\sum_{n=1}^{N} \sum_{k=1}^{K} \zeta_{n k}\left(\boldsymbol{\theta}^{\prime}\right) \log \pi_{k} \kappa_{k}\left(\boldsymbol{y}_{n} \mid \boldsymbol{\theta}\right),
\end{aligned}
$$

where $z_{n k}$ assigns the latent variable for the $n$th data point and $k$ th component in the mixture and $\zeta_{n k}\left(\theta^{\prime}\right)$ is its expected value under $p(z \mid y, \theta)$, given by

$$
\begin{aligned}
\zeta_{n k}\left(\boldsymbol{\theta}^{\prime}\right) & \triangleq \mathbb{E}_{\boldsymbol{\theta}^{\prime}}\left[z_{n k} \mid Y_{N}\right]=\sum_{Z_{N}} z_{n k} p_{N}\left(Z_{N} \mid Y_{N}, \boldsymbol{\theta}^{\prime}\right)=\sum_{Z_{N}} z_{n k} \prod_{j=1}^{N} p\left(z_{j} \mid y_{j}, \boldsymbol{\theta}^{\prime}\right) \\
& =\sum_{\boldsymbol{z}_{n}} z_{n k} p\left(\boldsymbol{z}_{n} \mid \boldsymbol{y}_{n}, \boldsymbol{\theta}^{\prime}\right)=\frac{\pi_{k} \kappa_{k}\left(y_{n} \mid \boldsymbol{\theta}^{\prime}\right)}{\sum_{j=1}^{K} \pi_{j} \kappa_{j}\left(\boldsymbol{y}_{n} \mid \boldsymbol{\theta}^{\prime}\right)} .
\end{aligned}
$$

where the second to last step follows since the $p(z \mid y, \theta)$ factorizes over $n$.

\section{M-step for a bias change in a Gaussian Mixture model}

The solution to the M-step depends on the form of the kernel function and on how the unknown parameters enter this function. Explicit solutions are given next for the Gaussian mixture model (GMM) based on $Y_{N}$ and with

$$
\theta=\Delta, \quad \kappa_{k}(y \mid \theta)=\kappa_{k}(y-\Delta)=\mathcal{N}\left(y-\Delta ; y_{k}^{0}, S\right) .
$$

The M-step can be found explicitly by finding the solution to $\frac{\partial}{\partial \Delta} Q\left(\Delta, \Delta^{\prime}\right)=0$. This gradient is given by

$$
\sum_{n=1}^{N} \sum_{k=1}^{K} \zeta_{n k}\left(\Delta^{\prime}\right)\left[\frac{\partial}{\partial \Delta} \log \kappa_{k}\left(y_{n}-\Delta\right)\right],
$$


where the term in brackets simplifies to

$$
\begin{array}{r}
\frac{\partial}{\partial \boldsymbol{\Delta}}\left\{-1 / 2\left[\left(y_{n}-y_{k}^{0}\right)-\Delta\right]^{T} S^{-1}\left[\left(y_{n}-y_{k}^{0}\right)-\Delta\right]\right\} \\
=\frac{\partial}{\partial \Delta}\left\{\Delta^{T} S^{-1}\left(y_{n}-y_{k}^{0}\right)-\frac{1}{2} \Delta^{T} S^{-1} \Delta\right\}=S^{-1}\left(y_{n}-y_{k}^{0}\right)-S^{-1} \Delta,
\end{array}
$$

giving the solution

$$
\Delta=\frac{\sum_{n=1}^{N} \sum_{k=1}^{K} \zeta_{n k}\left(\Delta^{\prime}\right)\left(y_{n}-y_{k}^{0}\right)}{\sum_{n=1}^{N} \sum_{k=1}^{K} \zeta_{n k}\left(\Delta^{\prime}\right)}=\frac{1}{N} \sum_{n=1}^{N} \sum_{k=1}^{K} \zeta_{n k}\left(\Delta^{\prime}\right)\left(y_{n}-y_{k}^{0}\right),
$$

where the last step follows since $\sum_{k=1}^{K} \zeta_{n k}\left(\Delta^{\prime}\right)=1$. The resulting iterates $\Delta^{(i)}$ produced from the EM algorithm are given in Algorithm 1 for a convergence criterion based on $\left\|\Delta^{(i)}-\Delta^{(i-1)}\right\|_{2}^{2}$. The algorithm can be initialized using (24), which, for the GMM, gives

$$
\Delta^{(0)}=\frac{1}{N} \sum_{n=1}^{N} y_{n}-\sum_{k=1}^{K} \pi_{k} y_{k}^{0}
$$

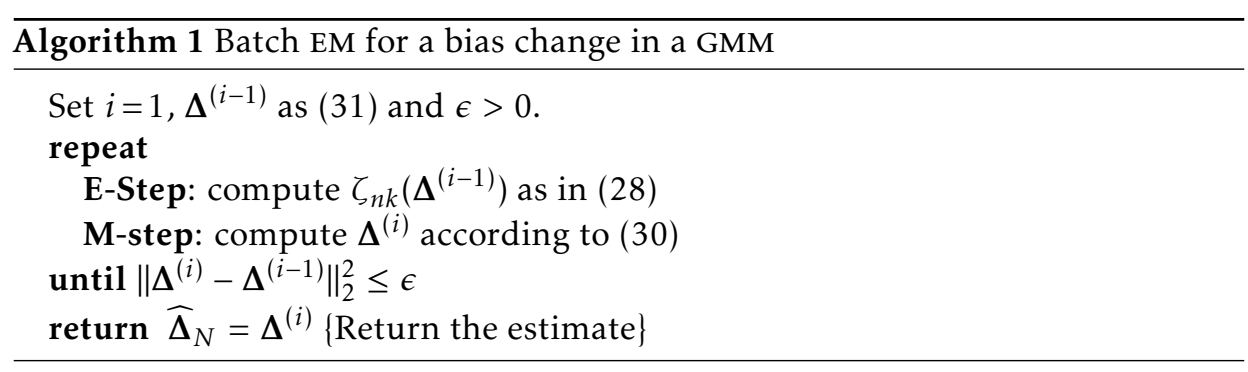

\subsection{Sequential estimation using stochastic approximation}

To evaluate the E-step in the EM algorithm, all measurements in $Y_{N}$ must be available and the EM algorithm is therefore a batch approach. A recursive version of EM was suggested by Cappé and Moulines (2009), based on a stochastic approximation of the E-step according to,

$$
\widetilde{Q}_{n}(\boldsymbol{\theta})=\gamma_{n} \mathbb{E}_{\widehat{\boldsymbol{\theta}}_{n-1}}\left[\log p(\boldsymbol{y}, \boldsymbol{z} \mid \boldsymbol{\theta}) \mid \boldsymbol{y}_{n}\right]+\left(1-\gamma_{n}\right) \widetilde{Q}_{n-1}(\boldsymbol{\theta}),
$$

where $\gamma_{n}$ is a forgetting factor, controlling the adaptation rate to incoming measurements. The M-step is unchanged and the estimate $\widehat{\boldsymbol{\theta}}_{n}$ is taken as the maximum of the $\widetilde{Q}_{n}$-function. Consistency and convergence rates for the estimator (32) are studied in Cappé and Moulines (2009). For consistency, $\gamma_{n}$ must be chosen such that

$$
0<\gamma_{n}<1, \quad \sum_{j=1}^{\infty} \gamma_{j}=\infty, \quad \sum_{j=1}^{\infty} \gamma_{j}^{2}<\infty
$$


To satisfy these conditions, the authors suggest the use of

$$
\gamma_{n}=\gamma_{0} n^{-\rho} \text {, }
$$

for $\gamma_{0} \in(0,1)$ and $\rho \in\left(\frac{1}{2}, 1\right]$. The particular choice $\gamma_{0}=\rho=1$ is equivalent to the recursion of Equation 12 given in Titterington (1984).

For mixture models, (32) follows as

$$
\widetilde{Q}_{n}(\boldsymbol{\theta})=\gamma_{n} \sum_{k=1}^{K} \zeta_{n k}\left(\widehat{\boldsymbol{\theta}}_{n-1}\right) \log \pi_{k} \kappa_{k}\left(y_{n} \mid \boldsymbol{\theta}\right)+\left(1-\gamma_{n}\right) \widetilde{Q}_{n-1}(\boldsymbol{\theta}),
$$

where $\zeta_{n k}(\cdot)$ is evaluated at the previous estimate $\widehat{\boldsymbol{\theta}}_{n-1}$. For a GMM as in (29), a recursive solution to the $\mathrm{M}$-step can be found. Starting with $\widetilde{Q}_{0}(\Delta)=-\frac{1}{2} \Delta^{T} S^{-1} \Delta$ and $\widehat{\Delta}_{0}=\mathbf{0}$ (no change), direct maximization of $\widetilde{Q}_{1}(\Delta), \widetilde{Q}_{2}(\Delta), \ldots, \widetilde{Q}_{n}(\Delta)$, for a sequence $\left\{y_{n}\right\}$ gives

$$
\widehat{\Delta}_{n}=\gamma_{n} \sum_{k=1}^{K} \zeta_{n k}\left(\widehat{\Delta}_{n-1}\right)\left(y_{n}-y_{k}^{0}\right)+\left(1-\gamma_{n}\right) \widehat{\Delta}_{n-1},
$$

see the Appendix for a proof. Similarly, to find $\widehat{\Delta}_{t: n}$ sequentially as described in Section 2.2, the recursion is

$$
\widehat{\Delta}_{t: n}=\gamma_{\widetilde{n}} \sum_{k=1}^{K} \zeta_{n k}\left(\widehat{\Delta}_{t: n-1}\right)\left(y_{n}-y_{k}^{0}\right)+\left(1-\gamma_{\widetilde{n}}\right) \widehat{\Delta}_{t: n-1},
$$

where $\widetilde{n}=n-t+1$. Recursion (35) gives rise to Algorithm 2, which produces an estimate $\widehat{\Delta}_{t: n}$ recursively at each new measurement $y_{n}$. The estimate $\widehat{\Delta}_{t: n}$ is used in sequential tests to compute the GLR statistic $\widetilde{s_{n}}$ in (9) where $\widehat{\Delta}_{t: n}$ needs to be found for $t \in\{1, \ldots, n\}$. That is, for each incoming data $y_{n}, n$ bias estimates are updated.

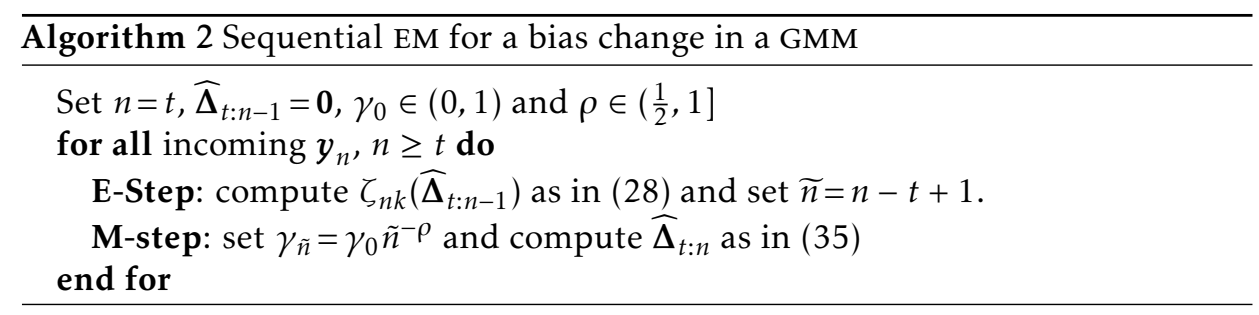

Notice that the computational complexities of Algorithms 1 and 2 are directly proportional to the number of kernels $K$. Therefore, the use of sparse models, such as the ones achieved by the GCE method, give the advantage of a reduced computation load. 


\section{Illustrative Examples}

\subsection{Simulation study}

The performance of statistical tests based on the approximated models (3) will depend on how close they are to true models (1). As noticed by Eguchi and Copas (2006), the power that is lost over all thresholds in a Neyman-Pearson test, i.e., based on a likelihood ratio statistic, when the true density for $\mathcal{H}^{1}, p^{1}(y)$, is misspecified by $\widehat{p}^{1}(y)$ is given by the relative entropy (Kullback-Leibler divergence) between the true and approximate densities, i.e.,

$$
\begin{array}{r}
\int_{-\infty}^{\infty} \operatorname{Pr}\left(s(y)=\log \frac{p^{1}(y)}{p^{0}(y)}>\hbar \mid \mathcal{H}^{1}\right)-\operatorname{Pr}\left(\widehat{s}(y)=\log \frac{\widehat{p}^{1}(y)}{p^{0}(y)}>\hbar, \mid \mathcal{H}^{1}\right) \mathrm{d} \hbar \\
=D_{\mathrm{KL}}\left(p^{1} \| \widehat{p}^{1}\right) \triangleq \int p^{1}(y) \log \frac{p^{1}(y)}{\widehat{p}^{1}(y)} \mathrm{d} y .
\end{array}
$$

A similar result is also given in case $\mathcal{H}^{0}$ is misspecified by $\widehat{p}^{0}(y)$, where the overall power loss is $D_{\mathrm{KL}}\left(p^{0} \| \widehat{p}^{0}\right)$. To illustrate the effects of different model approximations and potential gains of the suggested ideas, different approximations are evaluated for a selection of simulation problems. A total of seven problems are considered as described in Table 1. The densities for cases 1-6 were taken from Marron and Wand (1992); Botev et al. (2010). For each problem, a nominal dataset $Y_{N_{0}}^{0}$ is generated with $N_{0}=200$ samples. Three different approximations of $p^{0}(y)$ are considered based on the nominal dataset $Y_{N_{0}}^{0}$ :

- a Gaussian with parameters given from the standard maximum likelihood equations,

- a nonparametric model given by the KDE with a Gaussian kernel and bandwidth found using (16),

- a nonparametric model given by the GCE with a Gaussian kernel and an $\epsilon=10^{-8}$ approximation.

The true density for $\mathcal{H}^{1}$ is taken as $p^{0}(y-\Delta)$ where $\Delta=2$ for all cases. From $\mathcal{H}^{1}$, a test dataset $Y_{N}$ with $N=50$ samples is generated. The test data $Y_{N}$ is used for the estimation of $\Delta$ based on the three different models. For the Gaussian model, a maximum likelihood estimator is used, i.e., the estimate is the difference between the dataset averages $\widehat{\Delta}_{N}^{\text {Gauss }}=\bar{Y}_{N}-\bar{Y}_{N_{0}}^{0}$. For the KDE and GCE models, the parameter is estimated using the batch EM solution given in Algorithm 1 with initial value given by (31).

To analyze the effects of using the approximated models in statistical tests, the overall power losses given by $D_{\mathrm{KL}}\left(p^{0} \| \widehat{p}^{0}\right)$ and $D_{\mathrm{KL}}\left(p^{1} \| \widehat{p}^{1}\right)$ are computed, as well as the mean squared error (MSE) for the achieved estimates $\widehat{\Delta}_{N}$. The achieved values are shown in Table 1. As can be seen, the GCE estimates consistently give smaller values for the relative entropies and thus give GLR statistics that are closer to the optimal (achieved when the true model is used). This is the case even 
though the achieved estimates $\widehat{\Delta}_{N}$ do not always give the smallest MSE. The overall better performance of the GCE model can be motivated from its information theoretic derivation (see Botev and Kroese (2011)). Note that the performance of the KDE models could possibly be improved if a different bandwidth selection method was used. The resulting number of components $\left|\mathbb{K}_{\epsilon}\right|$ for the GCE estimates are $12,12,8,5,5,14,30$ for cases 1-7 respectively. Compared to the KDE, the reduced number of components for the GCE estimates considerably reduces the amount of computations needed to find the bias estimates.

\subsection{Batch detection of an increase in eruptions}

The Old Faithful geyser dataset (Azzalini and Bowman, 1990) is considered here to illustrate the methods for the batch multivariate case with known change time. The dataset contains 272 measurements with $d=2$ dimensions representing the registered length of the geyser's eruptions and the time between eruptions (both in minutes). A fraction $N_{0}=222$ of the measurements are used to estimate a density for the nominal model $\widehat{p}^{0}(y)$. As before, three different models are considered: a Gaussian model and nonparametric models given by the KDE and GCE methods with Gaussian kernels.

The measurements $Y_{N_{0}}^{0}$ are shown in Figure 1a together with contour lines for the density models. The components chosen for the GCE model are also shown in Figure 1a with a colormap relating to the weights $\pi^{*}$. With $K=32$, the GCE requires $86 \%$ less data to represent the density compared to the KDE. The GCE is also richer in details and with a tighter support compared to the KDE and Gaussian models.

A bias change is considered to illustrate the situation where the length of eruptions is increased by half a minute and the interval between them is reduced by 2 minutes, i.e., $\Delta=[0.5,-2]^{T}$. These values are added to the $N=50$ remaining measurements, which can be seen in Figure 1a. Using these abnormal measurements $Y_{N}, \Delta$ is estimated for the three different models in the same manner as discussed in Section 5.1 but the EM Algorithm 1 is initialized with $\Delta^{(0)}=[0,0]^{T}$ for a comparison. Notice the large bias for the estimate given by the Gaussian model. The iterates $\Delta^{(i)}$ are shown in Figure $1 \mathrm{~b}$ as a function of iterations. Due to the sparsity

of the GCE, $\widehat{\Delta}_{N}$ is computed 40 times faster compared to the estimate given by the KDE. After convergence of the iterates, the GLR statistic $\widehat{s_{N}}$ is computed for the different models, the values are 9.18, 21.71 and 83.71 for the Gaussian, KDE and GCE models, respectively. Based on the asymptotic expression (13a), a threshold $\hbar(0.01)=4.60$ is found. All tests can detect the change, although the one based on the GCE gives a much clearer response.

\subsection{Sequential detection of an increase of wear in an industrial robot joint}

By processing torque measurements collected from an industrial robot joint, a scalar quantity $y$ is generated to infer the mechanical condition of the joint gearbox (Bittencourt et al., 2014). The generated quantity $y$ is positive and remains 


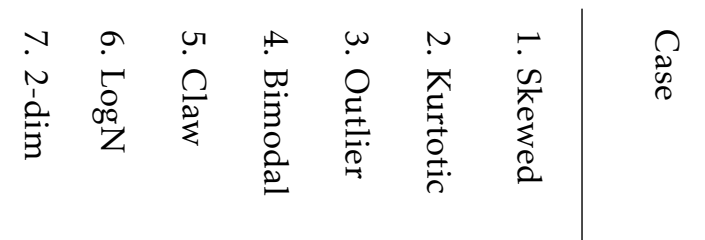

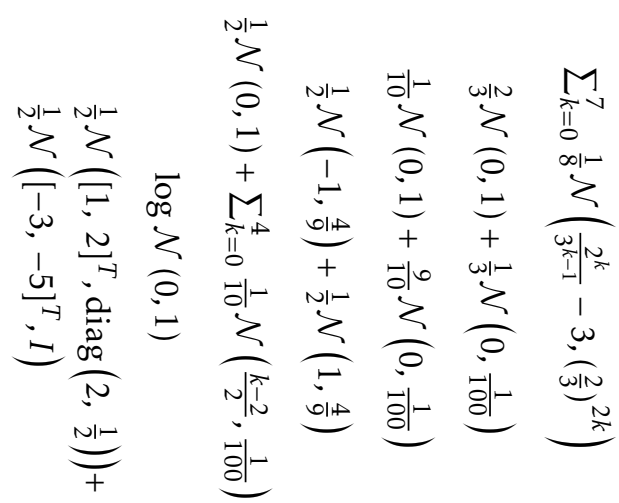

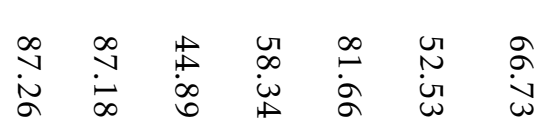

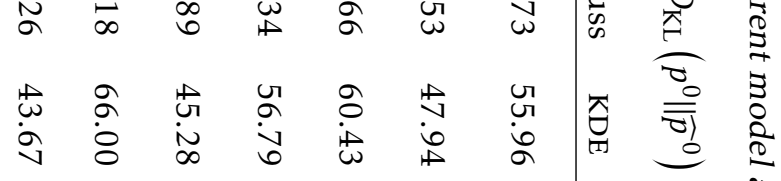

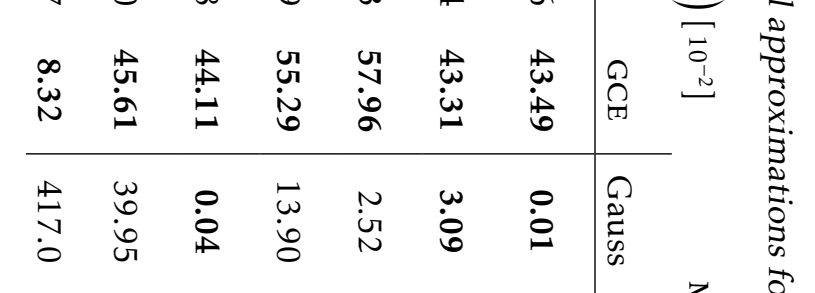

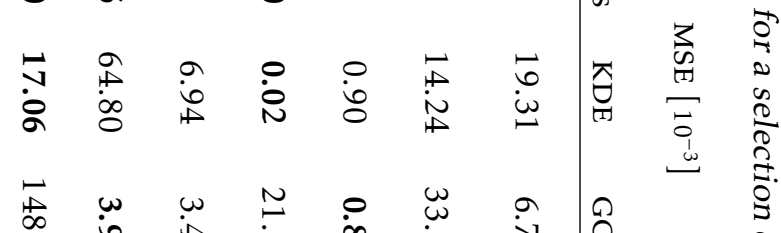

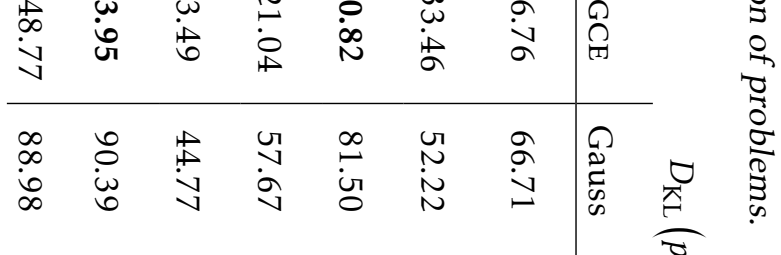

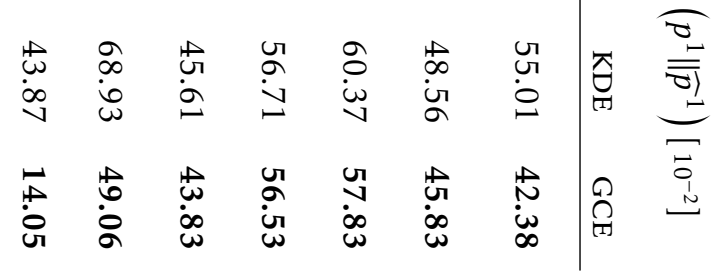




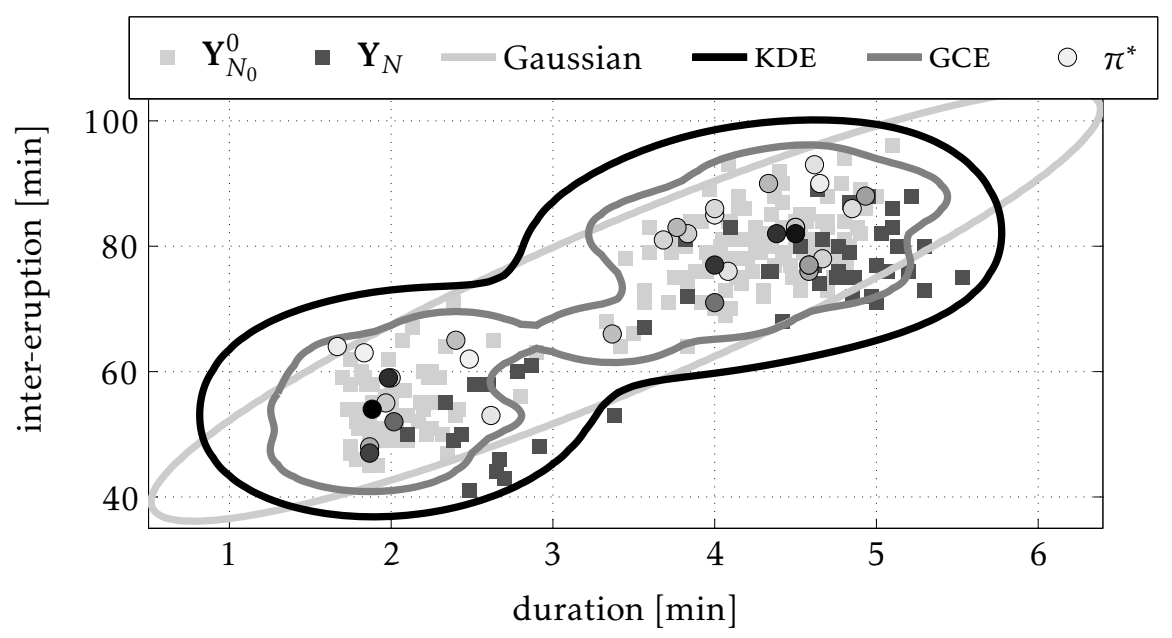

(a) Estimates of $p^{0}(y)$ and measurements.

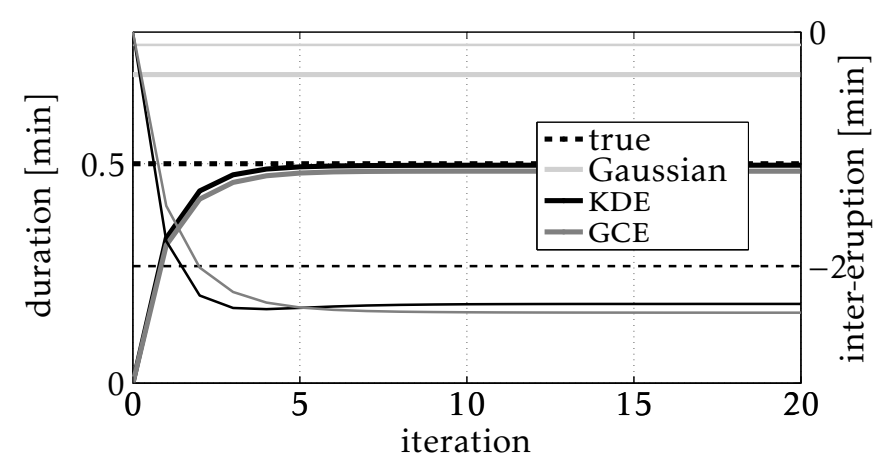

(b) Estimates of eruption duration (thick) and inter-eruption interval (thin). Notice the different axes.

Figure 1: GLR test for detection of eruptions increase in a geyser dataset. Notice how the test measurements $Y_{N}$ in Figure 1a overlap with the support for the nominal models. Despite this, a detection is achieved with any of the models, although the test based on the GCE gives a clearer response. 


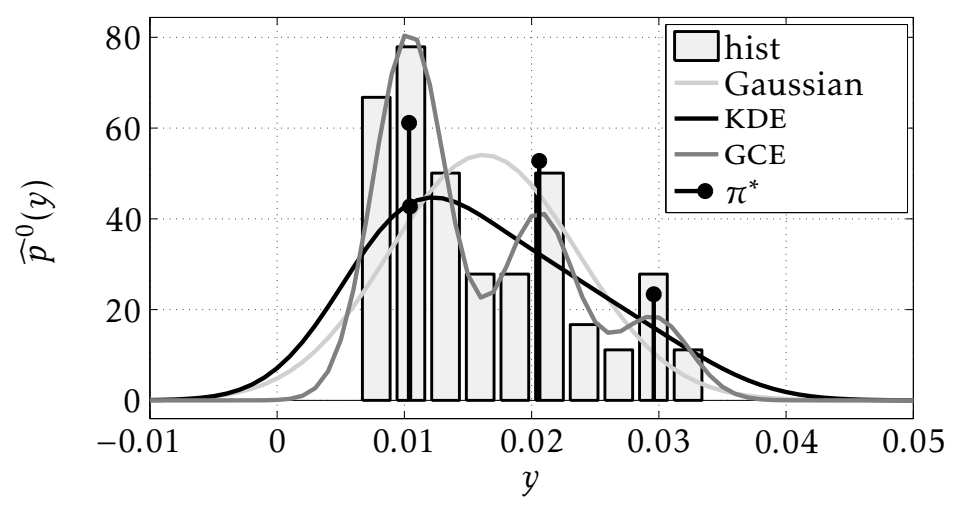

(a) Estimates of $p^{0}(y)$.

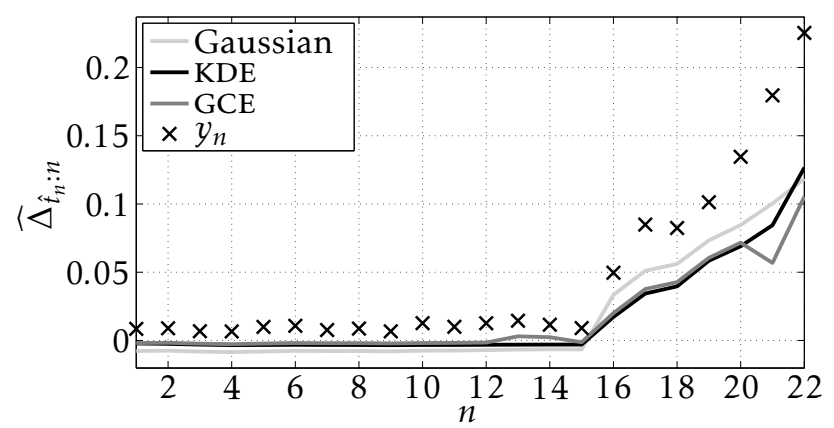

(b) Test data sequence $y_{n}$ and estimates $\widehat{\Delta}_{\widehat{t}_{n}: n}$.

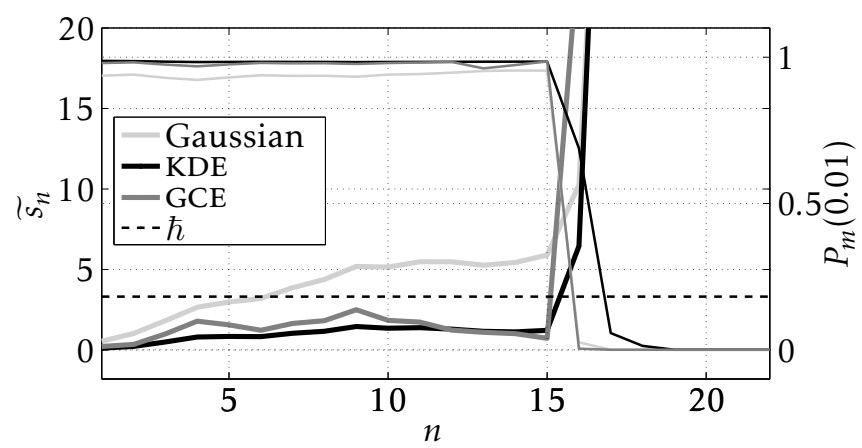

(c) GLR statistic $\widetilde{s_{n}}$ (thick) and $P_{m}(0.01)$ (thin). Notice the different axes.

Figure 2: GLR test for detection of abnormalities in the gearbox of a robot joint. Notice the false alarms triggered with the use of a Gaussian model. 
close to zero under normal conditions, deviating otherwise to indicate an anomaly. The data processing used in the generation of $y$ makes it difficult to determine its distribution function. From this application, it is however possible to collect nominal measurements before the application of the test. Based on $N_{0}=66$ nominal samples, the three models from Section 5.1 are considered. The resulting models and histogram of $Y_{N_{0}}^{0}$ can be seen in Figure 2a. The distribution of the measurements is multimodal and asymmetric, which makes the Gaussian model a poor representation with its mean falling in a region of the support with little data. The KDE estimate captures the asymmetry in the data, but not the multiple modes and presents a wide support; perhaps a more sophisticated choice of bandwidth would have improved the KDE estimate. The GCE estimate uses only four components, a reduction of $96 \%$ compared to the KDE. It also captures the multiple modes, asymmetry and has a tighter support.

Using these models, the objective is to detect a wear fault appearing around $t=$ 16 in a sequence $\left\{y_{n}\right\}$ with $1 \leq n \leq 22$. The change time is unknown and a sequential solution is sought as described in Sections. 2.1 and 2.2. To reduce the computational complexity, recursive maximum likelihood estimates $\widehat{\Delta}_{t: n}$ are found for $1 \leq t \leq n$. For the Gaussian model, the standard maximum likelihood estimate is used. Algorithm 2 is used for the KDE and GCE models with $\gamma_{0}=0.6$ and $\rho=1$. The data $\left\{y_{n}\right\}$ and the different estimates $\widehat{\Delta}_{\widehat{t}_{n}: n}$, with $\widehat{t}_{n}$ given in (10), are shown in Figure $2 \mathrm{~b}$. Up to $n=16, y_{n}$ has values smaller than the mean for the Gaussian model making the estimate deviate towards negative values for this model.

The resulting models are used to find the GLR statistic $\widetilde{s_{n}}$ as given in (9), these are shown in Figure $2 c$ together with the threshold $\hbar(0.01)=3.32$ found according to (13a). The Gaussian model generates false alarms from $n=7$ and the KDE and GCE based tests detect a change from $n=16$. The error probabilities $P_{m}(0.01)$ given by (13b) are found based on numerical evaluation of the Fisher information, the values are also shown in Figure $2 c$ as a function of $n$. As can be seen, there is a sharp decay of $P_{m}$ for the Gaussian and GCE models. The Gaussian model gives smaller values of $P_{m}$ before the change. The value for $P_{m}$ achieved with the KDE decays more slowly compared to the others, with a value of 0.73 at $n=16$.

In this application, an early detection is very important to allow for condition based maintenance, giving enough time to perform maintenance. To decide for maintenance actions, it is also critical to have few false alarms and that the detection error $P_{m}$ can be used to support service decisions. In this application, the test obtained using the GCE model presented the best compromise for these requirements.

\section{Conclusions and Future Work}

This paper proposed a two step approach for anomaly detection using a bias change model and GLR tests. In the first step, a model for the normality is found based on a nominal dataset. Nonparametric density estimates are used which 
give high flexibility since specification of a density function is not needed. In the second step, maximum likelihood estimates of a bias change are computed using the EM algorithm. The use of a sparse density model can considerably reduce the computations needed for the estimators. The density model and bias change estimate are then used in GLR tests to decide whether an abnormality is present or not. Both batch and sequential cases are considered and the approaches only require availability of nominal data and minimal/meaningful specification in terms of a desired probability of false alarm (to find a threshold). Using available asymptotic expressions for the GLR statistic, it is also possible to give estimates of the uncertainties associated with the decision, which are important to support higher level decisions. The approaches were illustrated in simulations and real data examples including the detection of an increase of eruptions in a geyser and of a wear fault in an industrial robot joint. The results achieved show clear improvements compared to tests based on a Gaussian assumption.

Currently, the decision errors are estimated based on asymptotic expressions which may differ for a finite number of measurements. In this direction, it would be interesting to study approaches to provide estimates for the finite sample behavior of the error probabilities. This could possibly lead to the derivation of adaptive thresholds and more accurate error estimates.

\section{Appendix}

\section{Proof of (22)}

Let $[C]_{i j} \triangleq \int_{\mathbb{R}^{d}} \mathcal{N}\left(y ; y_{i}, S\right) \mathcal{N}\left(y ; y_{i}, S\right) \mathrm{d} y, P \triangleq S^{-1}$ and $c=(2 \pi)^{-d / 2}|S|^{-1 / 2}$ then

$$
[C]_{i j}=c \int e^{-\frac{1}{2}\left\{\left[\boldsymbol{y}-\boldsymbol{y}_{i}\right]^{T} P\left[\boldsymbol{y}-\boldsymbol{y}_{i}\right]+\left[\boldsymbol{y}-\boldsymbol{y}_{j}\right]^{T} P\left[\boldsymbol{y}-\boldsymbol{y}_{j}\right]\right\}} \mathrm{d} \boldsymbol{y} .
$$

Using weighted inner product notation, the term in curly brackets is written as $\left\langle y-y_{i}, y-y_{i}\right\rangle_{P}+\left\langle y-y_{j}, y-y_{j}\right\rangle_{P}$ and simplifies to

$$
\begin{aligned}
2\langle y, y\rangle_{P}-2\left\langle y, y_{i}\right. & \left.+y_{j}\right\rangle_{P}+\left\langle y_{i}, y_{i}\right\rangle_{P}+\left\langle y_{j}, y_{j}\right\rangle_{P} \\
+\left(\frac { 1 } { 2 } \left\langley_{i}+y_{j},\right.\right. & \left.\left.y_{i}+y_{j}\right\rangle_{P}-\frac{1}{2}\left\langle y_{i}+y_{j}, y_{i}+y_{j}\right\rangle_{P}\right) \\
& =\left\langle y-\frac{y_{i}+y_{j}}{2}, y-\frac{y_{i}+y_{j}}{2}\right\rangle_{2 P}+\left\langle y_{i}-y_{j}, y_{i}-y_{j}\right\rangle_{P / 2} .
\end{aligned}
$$

Rearranging $c$ and taking the integral gives the result

$$
[C]_{i j}=\mathcal{N}\left(y_{i} ; y_{j}, 2 S\right) \int \mathcal{N}\left(y ; \frac{y_{i}+y_{j}}{2}, S / 2\right) \mathrm{d} y=\mathcal{N}\left(y_{i} ; y_{j}, 2 S\right) .
$$




\section{Proof of (34)}

We show the results for $n=1$ and $n=2$, the remaining follows by induction. Let $P \triangleq S^{-1}, \widetilde{Q}_{0}(\Delta)=-\frac{1}{2} \Delta^{T} P \Delta=-\frac{1}{2}\|\Delta\|_{P}^{2}, \widehat{\Delta}_{0}=\mathbf{0}$, and (29), then (33) gives

$$
\begin{gathered}
\widetilde{Q}_{1}(\Delta) \propto-\frac{1}{2} \gamma_{1} \sum_{k=1}^{K} \zeta_{1 k}\left(\widehat{\Delta}_{0}\right)\left\|\left(y_{1}-y_{k}\right)-\Delta\right\|_{P}^{2}-\frac{1}{2}\left(1-\gamma_{1}\right)\|\Delta\|_{P}^{2} \\
\left.\frac{\partial}{\partial \Delta} \widetilde{Q}_{1}(\Delta)=\gamma_{1} \sum_{k=1}^{K} \zeta_{1 k}\left(\widehat{\Delta}_{0}\right) P\left[\left(y_{1}-y_{k}\right)-\Delta\right)\right]-\left(1-\gamma_{1}\right) P \Delta
\end{gathered}
$$

and therefore $\widehat{\Delta}_{1}=\gamma_{1} \sum_{k=1}^{K} \zeta_{1 k}\left(\widehat{\Delta}_{0}\right)\left(y_{1}-y_{k}\right)$. Similarly, for $n=2$

$$
\begin{aligned}
& \left.\frac{\partial}{\partial \Delta} \widetilde{Q}_{2}(\Delta)=\gamma_{2} \sum_{k=1}^{K} \zeta_{2 k}\left(\widehat{\Delta}_{1}\right) P\left[\left(y_{2}-y_{k}\right)-\Delta\right)\right] \\
& \left.+\left(1-\gamma_{2}\right)\left[\gamma_{1} \sum_{k=1}^{K} \zeta_{1 k}\left(\widehat{\Delta}_{0}\right) P\left[\left(y_{1}-y_{k}\right)-\Delta\right)\right]-\left(1-\gamma_{1}\right) P \Delta\right]
\end{aligned}
$$

which gives $\widehat{\Delta}_{2}=\gamma_{2} \sum_{k=1}^{K} \zeta_{2 k}\left(\widehat{\Delta}_{1}\right)\left(y_{2}-y_{k}^{0}\right)+\left(1-\gamma_{2}\right) \widehat{\Delta}_{1}$. 


\section{Bibliography}

D. Agarwal. Detecting anomalies in cross-classified streams: a Bayesian approach. Knowledge and Information Systems, 11(1):29-44, 2007.

A. Azzalini and A. W. Bowman. A look at some data on the Old Faithful Geyser. Journal of the Royal Statistical Society. Series C (Applied Statistics), 39(3):357365, 1990.

M. Basseville and I. V. Nikiforov. Detection of abrupt changes: theory and application. Prentice-Hall, Inc., Upper Saddle River, NJ, USA, 1993.

J. A. Bilmes. A gentle tutorial of the EM algorithm and its application to parameter estimation for Gaussian mixture and hidden Markov models. Technical Report TR-97-021, International Computer Science Institute, Berkeley, California, May 1997.

C. M. Bishop. Pattern Recognition and Machine Learning. Springer, New York, USA, 1st edition, 2006.

A. C. Bittencourt, K. Saarinen, S. Sander-Tavallaey, S. Gunnarsson, and M. Norrlöf. A data-driven approach to diagnostics of repetitive processes in the distribution domain - Applications to gearbox diagnostics in industrial robots and rotating machines. Mechatronics, -(0):-, 2014. available online.

Z. Botev and D. Kroese. The generalized cross entropy method, with applications to probability density estimation. Methodology and Computing in Applied Probability, 13:1-27, 2011.

Z. I. Botev, J. F. Grotowski, and D. P. Kroese. Kernel density estimation via diffusion. The Annals of Statistics, 38(5):2916-2957, 2010.

T. Cacoullos. Estimation of a multivariate density. Annals of the Institute of Statistical Mathematics, 18:179-189, 1966.

O. Cappé and E. Moulines. On-line expectation-maximization algorithm for latent data models. Journal of the Royal Statistical Society: Series B (Statistical Methodology), 71(3):593-613, 2009.

V. Chandola, A. Banerjee, and V. Kumar. Anomaly detection: A survey. ACM Computing Surveys (CSUR), 41(3):15, 2009.

A. P. Dempster, N. M. Laird, and D. B. Rubin. Maximum likelihood from incomplete data via the EM algorithm. Journal of the Royal Statistical Society. Series B (Methodological), 39(1):1-38, 1977.

M. Desforges, P. Jacob, and J. Cooper. Applications of probability density estimation to the detection of abnormal conditions in engineering. Proceedings of the Institution of Mechanical Engineers, Part C: Journal of Mechanical Engineering Science, 212(8):687-703, 1998. 
L. Devroye and G. L. Wise. Detection of abnormal behavior via nonparametric estimation of the support. SIAM Journal on Applied Mathematics, 38(3):480$488,1980$.

S. Eguchi and J. Copas. Interpreting Kullback-Leibler divergence with the Neyman-Pearson lemma. Journal of Multivariate Analysis, 97(9):2034-2040, 2006.

F. Gustafsson. Adaptive Filtering and Change Detection. Wiley, October 2000.

R. Isermann. Fault-Diagnosis Systems - An Introduction from Fault Detection to Fault Tolerance. Springer, 1st edition, 2006.

C. Jones, J. Marron, and S. Sheather. Progress in data-based bandwidth selection for kernel density estimation. Computational Statistics, (11):337-381, 1996a.

M. C. Jones, J. S. Marron, and S. J. Sheather. A brief survey of bandwidth selection for density estimation. Journal of the American Statistical Association, 91(433): 401-407, 1996b.

D. J. C. Mackay. Information Theory, Inference and Learning Algorithms. Cambridge University Press, 1st edition, June 2003.

J. S. Marron and M. P. Wand. Exact mean integrated squared error. The Annals of Statistics, 20(2):712-736, 1992.

J. Neyman and E. S. Pearson. On the problem of the most efficient tests of statistical hypotheses. Philosophical Transactions of the Royal Society of London. Series A, Containing Papers of a Mathematical or Physical Character, 231:289337, 1933.

E. Parzen. On estimation of a probability density function and mode. The Annals of Mathematical Statistics, 33(3):1065-1076, 1962.

B. Schölkopf, J. C. Platt, J. Shawe-Taylor, A. J. Smola, and R. C. Williamson. Estimating the support of a high-dimensional distribution. Neural computation, 13(7):1443-1471, 2001.

B. W. Silverman. Density estimation for statistics and data analysis, volume 26. Chapman \& Hall/CRC, 1986.

D. M. Titterington. Recursive parameter estimation using incomplete data. Journal of the Royal Statistical Society. Series B (Methodological), 46(2):257-267, 1984.

H. L. Van Trees. Detection, Estimation and Modulation Theory, Part I. Wiley, New York, 1st edition, 2001.

V. N. Vapnik and S. Mukherjee. Support vector method for multivariate density estimation. In Advances in Neural Information Processing Systems (NIPS), pages 659-665. MIT Press, 2000. 
A. Wald. Sequential tests of statistical hypotheses. The Annals of Mathematical Statistics, 16(2):117-186, 1945.

D.-Y. Yeung and C. Chow. Parzen-window network intrusion detectors. In Proceedings of the 16th International Conference on Pattern Recognition, volume 4, pages 385-388, Quebec, Canada, 2002. IEEE. 


\section{PhD Dissertations \\ Division of Automatic Control \\ Linköping University}

M. Millnert: Identification and control of systems subject to abrupt changes. Thesis No. 82, 1982. ISBN 91-7372-542-0.

A. J. M. van Overbeek: On-line structure selection for the identification of multivariable systems. Thesis No. 86, 1982. ISBN 91-7372-586-2.

B. Bengtsson: On some control problems for queues. Thesis No. 87, 1982. ISBN 91-7372593-5.

S. Ljung: Fast algorithms for integral equations and least squares identification problems. Thesis No. 93, 1983. ISBN 91-7372-641-9.

H. Jonson: A Newton method for solving non-linear optimal control problems with general constraints. Thesis No. 104, 1983. ISBN 91-7372-718-0.

E. Trulsson: Adaptive control based on explicit criterion minimization. Thesis No. 106, 1983. ISBN 91-7372-728-8.

K. Nordström: Uncertainty, robustness and sensitivity reduction in the design of single input control systems. Thesis No. 162, 1987. ISBN 91-7870-170-8.

B. Wahlberg: On the identification and approximation of linear systems. Thesis No. 163, 1987. ISBN 91-7870-175-9.

S. Gunnarsson: Frequency domain aspects of modeling and control in adaptive systems. Thesis No. 194, 1988. ISBN 91-7870-380-8.

A. Isaksson: On system identification in one and two dimensions with signal processing applications. Thesis No. 196, 1988. ISBN 91-7870-383-2.

M. Viberg: Subspace fitting concepts in sensor array processing. Thesis No. 217, 1989. ISBN 91-7870-529-0.

K. Forsman: Constructive commutative algebra in nonlinear control theory. Thesis No. 261, 1991. ISBN 91-7870-827-3.

F. Gustafsson: Estimation of discrete parameters in linear systems. Thesis No. 271, 1992. ISBN 91-7870-876-1.

P. Nagy: Tools for knowledge-based signal processing with applications to system identification. Thesis No. 280, 1992. ISBN 91-7870-962-8.

T. Svensson: Mathematical tools and software for analysis and design of nonlinear control systems. Thesis No. 285, 1992. ISBN 91-7870-989-X.

S. Andersson: On dimension reduction in sensor array signal processing. Thesis No. 290, 1992. ISBN 91-7871-015-4.

H. Hjalmarsson: Aspects on incomplete modeling in system identification. Thesis No. 298, 1993. ISBN 91-7871-070-7.

I. Klein: Automatic synthesis of sequential control schemes. Thesis No. 305, 1993. ISBN 91-7871-090-1.

J.-E. Strömberg: A mode switching modelling philosophy. Thesis No. 353, 1994. ISBN 91 7871-430-3.

K. Wang Chen: Transformation and symbolic calculations in filtering and control. Thesis No. 361, 1994. ISBN 91-7871-467-2.

T. McKelvey: Identification of state-space models from time and frequency data. Thesis No. 380, 1995. ISBN 91-7871-531-8.

J. Sjöberg: Non-linear system identification with neural networks. Thesis No. 381, 1995. ISBN 91-7871-534-2.

R. Germundsson: Symbolic systems - theory, computation and applications. Thesis No. 389, 1995. ISBN 91-7871-578-4. 
P. Pucar: Modeling and segmentation using multiple models. Thesis No. 405, 1995. ISBN 91-7871-627-6.

H. Fortell: Algebraic approaches to normal forms and zero dynamics. Thesis No. 407, 1995. ISBN 91-7871-629-2.

A. Helmersson: Methods for robust gain scheduling. Thesis No. 406, 1995. ISBN 91-7871628-4.

P. Lindskog: Methods, algorithms and tools for system identification based on prior knowledge. Thesis No. 436, 1996. ISBN 91-7871-424-8.

J. Gunnarsson: Symbolic methods and tools for discrete event dynamic systems. Thesis No. 477, 1997. ISBN 91-7871-917-8.

M. Jirstrand: Constructive methods for inequality constraints in control. Thesis No. 527, 1998. ISBN 91-7219-187-2.

U. Forssell: Closed-loop identification: Methods, theory, and applications. Thesis No. 566, 1999. ISBN 91-7219-432-4.

A. Stenman: Model on demand: Algorithms, analysis and applications. Thesis No. 571, 1999. ISBN 91-7219-450-2.

N. Bergman: Recursive Bayesian estimation: Navigation and tracking applications. Thesis No. 579, 1999. ISBN 91-7219-473-1.

K. Edström: Switched bond graphs: Simulation and analysis. Thesis No. 586, 1999. ISBN 91-7219-493-6.

M. Larsson: Behavioral and structural model based approaches to discrete diagnosis. Thesis No. 608, 1999. ISBN 91-7219-615-5.

F. Gunnarsson: Power control in cellular radio systems: Analysis, design and estimation. Thesis No. 623, 2000. ISBN 91-7219-689-0.

V. Einarsson: Model checking methods for mode switching systems. Thesis No. 652, 2000. ISBN 91-7219-836-2.

M. Norrlöf: Iterative learning control: Analysis, design, and experiments. Thesis No. 653, 2000. ISBN 91-7219-837-0.

F. Tjärnström: Variance expressions and model reduction in system identification. Thesis No. 730, 2002. ISBN 91-7373-253-2.

J. Löfberg: Minimax approaches to robust model predictive control. Thesis No. 812, 2003. ISBN 91-7373-622-8.

J. Roll: Local and piecewise affine approaches to system identification. Thesis No. 802, 2003. ISBN 91-7373-608-2.

J. Elbornsson: Analysis, estimation and compensation of mismatch effects in A/D converters. Thesis No. 811, 2003. ISBN 91-7373-621-X.

O. Härkegård: Backstepping and control allocation with applications to flight control. Thesis No. 820, 2003. ISBN 91-7373-647-3.

R. Wallin: Optimization algorithms for system analysis and identification. Thesis No. 919, 2004. ISBN 91-85297-19-4.

D. Lindgren: Projection methods for classification and identification. Thesis No. 915, 2005. ISBN 91-85297-06-2.

R. Karlsson: Particle Filtering for Positioning and Tracking Applications. Thesis No. 924, 2005. ISBN 91-85297-34-8.

J. Jansson: Collision Avoidance Theory with Applications to Automotive Collision Mitigation. Thesis No. 950, 2005. ISBN 91-85299-45-6.

E. Geijer Lundin: Uplink Load in CDMA Cellular Radio Systems. Thesis No. 977, 2005. ISBN 91-85457-49-3.

M. Enqvist: Linear Models of Nonlinear Systems. Thesis No. 985, 2005. ISBN 91-8545764-7.

T. B. Schön: Estimation of Nonlinear Dynamic Systems - Theory and Applications. Thesis No. 998, 2006. ISBN 91-85497-03-7. 
I. Lind: Regressor and Structure Selection - Uses of ANOVA in System Identification. Thesis No. 1012, 2006. ISBN 91-85523-98-4.

J. Gillberg: Frequency Domain Identification of Continuous-Time Systems Reconstruction and Robustness. Thesis No. 1031, 2006. ISBN 91-85523-34-8.

M. Gerdin: Identification and Estimation for Models Described by Differential-Algebraic Equations. Thesis No. 1046, 2006. ISBN 91-85643-87-4.

C. Grönwall: Ground Object Recognition using Laser Radar Data - Geometric Fitting, Performance Analysis, and Applications. Thesis No. 1055, 2006. ISBN 91-85643-53-X.

A. Eidehall: Tracking and threat assessment for automotive collision avoidance. Thesis No. 1066, 2007. ISBN 91-85643-10-6.

F. Eng: Non-Uniform Sampling in Statistical Signal Processing. Thesis No. 1082, 2007. ISBN 978-91-85715-49-7.

E. Wernholt: Multivariable Frequency-Domain Identification of Industrial Robots. Thesis No. 1138, 2007. ISBN 978-91-85895-72-4.

D. Axehill: Integer Quadratic Programming for Control and Communication. Thesis No. 1158, 2008. ISBN 978-91-85523-03-0.

G. Hendeby: Performance and Implementation Aspects of Nonlinear Filtering. Thesis No. 1161, 2008. ISBN 978-91-7393-979-9.

J. Sjöberg: Optimal Control and Model Reduction of Nonlinear DAE Models. Thesis No. 1166, 2008. ISBN 978-91-7393-964-5.

D. Törnqvist: Estimation and Detection with Applications to Navigation. Thesis No. 1216, 2008. ISBN 978-91-7393-785-6.

P-J. Nordlund: Efficient Estimation and Detection Methods for Airborne Applications. Thesis No. 1231, 2008. ISBN 978-91-7393-720-7.

H. Tidefelt: Differential-algebraic equations and matrix-valued singular perturbation. Thesis No. 1292, 2009. ISBN 978-91-7393-479-4.

H. Ohlsson: Regularization for Sparseness and Smoothness - Applications in System Identification and Signal Processing. Thesis No. 1351, 2010. ISBN 978-91-7393-287-5.

S. Moberg: Modeling and Control of Flexible Manipulators. Thesis No. 1349, 2010. ISBN 978-91-7393-289-9.

J. Wallén: Estimation-based iterative learning control. Thesis No. 1358, 2011. ISBN 978 91-7393-255-4.

J. Hol: Sensor Fusion and Calibration of Inertial Sensors, Vision, Ultra-Wideband and GPS. Thesis No. 1368, 2011. ISBN 978-91-7393-197-7.

D. Ankelhed: On the Design of Low Order H-infinity Controllers. Thesis No. 1371, 2011. ISBN 978-91-7393-157-1.

C. Lundquist: Sensor Fusion for Automotive Applications. Thesis No. 1409, 2011. ISBN 978-91-7393-023-9.

P. Skoglar: Tracking and Planning for Surveillance Applications. Thesis No. 1432, 2012. ISBN 978-91-7519-941-2.

K. Granström: Extended target tracking using PHD filters. Thesis No. 1476, 2012. ISBN 978-91-7519-796-8.

C. Lyzell: Structural Reformulations in System Identification. Thesis No. 1475, 2012. ISBN 978-91-7519-800-2.

J. Callmer: Autonomous Localization in Unknown Environments. Thesis No. 1520, 2013. ISBN 978-91-7519-620-6.

D. Petersson: A Nonlinear Optimization Approach to H2-Optimal Modeling and Control. Thesis No. 1528, 2013. ISBN 978-91-7519-567-4.

Z. Sjanic: Navigation and Mapping for Aerial Vehicles Based on Inertial and Imaging Sensors. Thesis No. 1533, 2013. ISBN 978-91-7519-553-7. 
F. Lindsten: Particle Filters and Markov Chains for Learning of Dynamical Systems. Thesis No. 1530, 2013. ISBN 978-91-7519-559-9.

P. Axelsson: Sensor Fusion and Control Applied to Industrial Manipulators. Thesis No. 1585, 2014. ISBN 978-91-7519-368-7. 